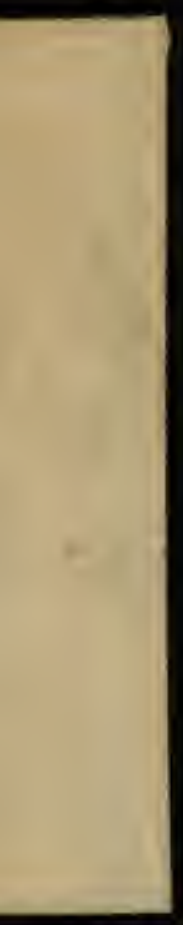




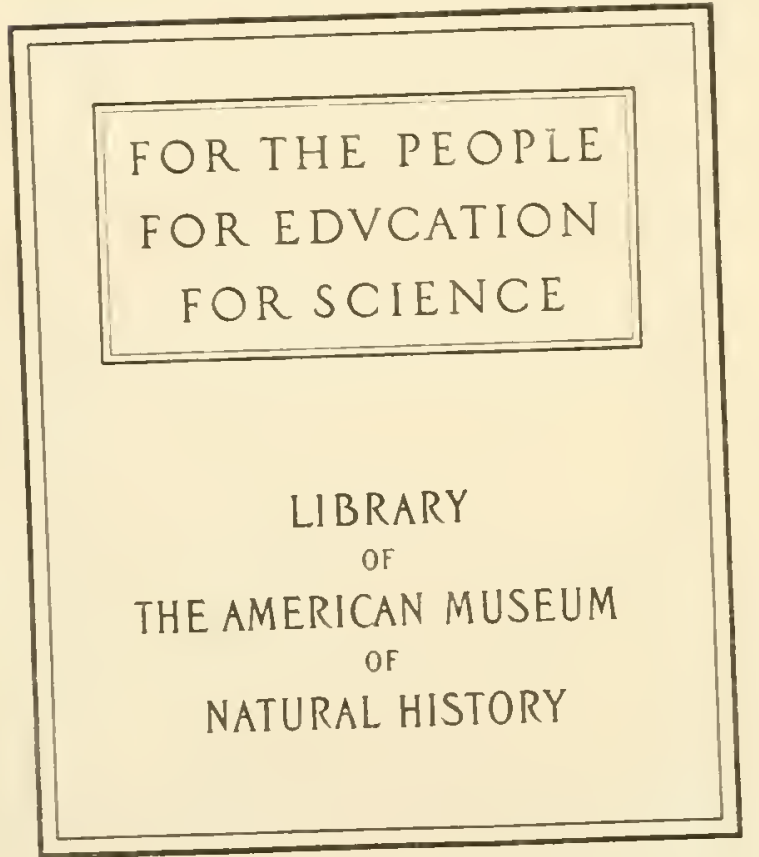






\title{
(i) ecasional fiapers \\ OF THE
}

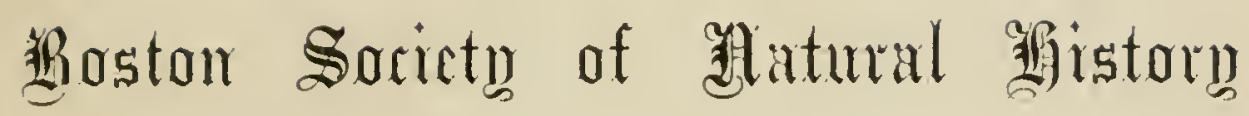

VII.

\section{FAUNA OF NEW ENGLAND.}

\section{1.}

LIST OF THE AVES.

By GLOVER M. ALLEN.

\author{
BOSTON : \\ PRINTED FOR THE SOCIETY FROM THE \\ GURDON SALTONSTALL FUND. \\ JUNE, 1909.
}





\section{Fauna of New England.}

'The Library and Publishing Committee will issue, as volume seren of the Occasional Papers, a Catalogue of the animals of New Fngland.

Parts will be published at irregular intervils, and though the details of the several lists will vary somewhat in the different groups, each list will inclucle, 1st, the accepted name (scientific and vernacular); 2d, reference to the original description, with record of locality; $3 d$, reference to an anthentic description and illustration, and the habitat and occurrence.

The Committee considers these lists a necessary preliminary to a series of comprehensive, illustrated monographs, the publication of which it is hoped the Societry will at some later time take upon itself.

The Committee also belieres that these lists will aid in the accumulation of raluable material for the New England collection of the Society. With this in view a separate check list of names will be issued with each part. In this check list the + indicates a specimen in the Socioty's Museum; the - that a trustwortly record exists.

'Towarl the accomplishment of its aims the Committee invites the coöperation of all interested in the Fanna of New England.

Communications concerning specimens should be addressed to the ('rikator of the Society (Chias. WV. Johnson), notes and records of orcurrence to the Entron of the "Fauna of New England" (Sammel Henshaw).

Boston Society Natural History.

2:34 Berkeley St., Boston, Mass. 



\section{AVES.}

The following list aims to include all species of birds known to have occurred in New England within historic times. Those only whose occurrence is believed to be natural are given a serial number, while introduced, escaped, or hypothetieal species are given in a separate list. The nomenclature is that adopted by the American ornithologists' union, including the changes of the 14th supplement to the Check-List, July, 1908. Of the vernaeular names, the first given is in each case that of the Check-List, and those following are local names eurrent for the most part in New England. Unless otherwise stated, the term "migrant" means that the species occurs in both the spring and the fall migrations; "summer resident" implies that the speeies breeds; "resident," that indiridual birds remain the entire year in the State. 'The migration and nesting dates given, are almost wholly those available from published sources and are hence in many cases, somewhat meager. Unusual dates are enclosed within parentheses. If the records for the presence of a given species within any State do not exceed three, the place and date of each are given if known.

For brevity of citation, references to figures of eggs in the works of Bendire, Capen, and Seebohm are giren by the name of the author followed by the date, plate, and figure. The titles of these works follow: -

Bendire, C. Life histories of North American birds with special reference to their breeding habits and eggs. Special bull. no. 1, U. S. nat. mus., 1592 , viii +446 p., 12 pls.; special bull. no. 3 , U. S. nat. mus., 1895, ix +518 pp., 7 pls.

Capen, Elwin A. Oology of New England: a description of the eggs, nests, and breeding habits of the birds known to breed in New England, with colored illustrations of their eggs. Boston, 1SS6, 4to, 116 pp., 25 pls.

Secbohm, Henry. Coloured figures of the eggs of British birds, with descriptive notices. Sheffield, xxiv +304 pp., pls. 

Colỵmbus holboellii auritus

Podilymbus podiceps

Gavia immer arctica stellata

Lunda cirrhata

Fratercula arctica

Cepphus grrylle

Uria troile. lomvia

Alca torda

Plautus impennis

Alle alle

Megalestris skua

Stercorarius pomarinus parasiticus

Pagophila alba

longicaudus

Rissa tridactrla .

Larus hrperboreus

$$
\begin{aligned}
& \text { leucopterus . } \\
& \text { kumlieni . } \\
& \text { marinus . } \\
& \text { argentatus } \\
& \text { delawarensis } \\
& \text { phicilladelphia. }
\end{aligned}
$$

Xema sabinii

Gelochelidon nilotica

Sterna caspia maxima

sandricensis acuflarida forsteri hirundo . paradisaea dougalli antillarum
Me N.H. V. Mass R. I Conn.

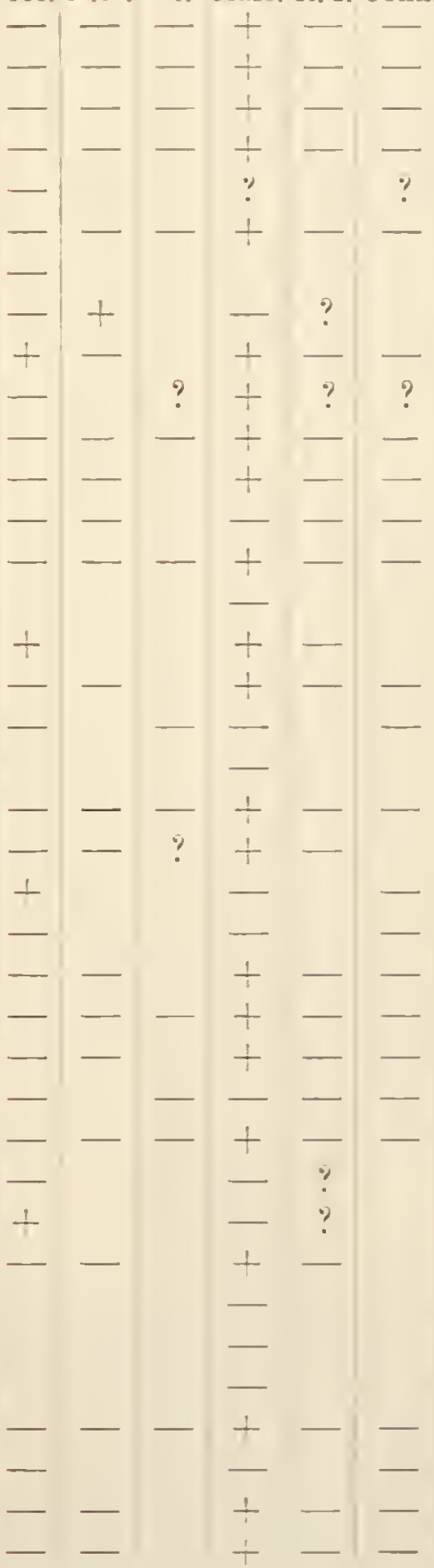


Sterna fusciata

Hydrochelidon nigra surinamensis

Rynchops nigral

Fulmarus glacialis.

Puffinus borealis gravis griseus

Acstrelata hasitata

Daption capensis

Oceanodroma lencorhoa

Oceanites oceanicus

Sula leucogastra bassana

Phalacrocorax carbo auritus

Pelecanus erythrorhynchos occidentalis

Fregata aquila

Mergus americanus serrator

Lophodytes cucullatus

Anas platyrhynchos mobripes

Chaulelasmus streperus

Marecal penelope americana

Nettion crecea carolinensis

Querquedula discors

Spatula clypeata

Dafila acuta

Aix sponsa

Marila americana vallisneria marila

affinis collaris

Clangula clangula americana islandica

Charitonetta albeola

Harelda hyemalis

Histrionicus histrionicus

Camptorhynchus labradorius
Me. N.H. Vt. Mass. R. I. Conn.

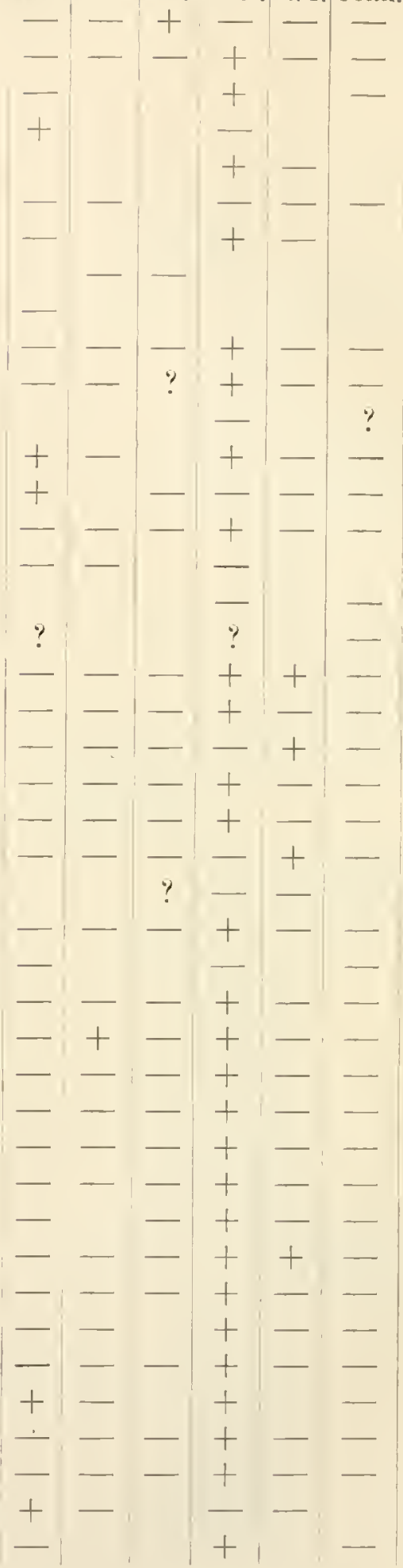


Somateria mollissima borealis dresseri spectabilis

Oidemia americana deglandi perspicillata

Erismatura jamaicensis Nomonyx dominicus Chen hyperborea

cacrulescens Anser albifrons gambeli Branta canadensis

hutchinsii bernicla glaucogastra nigricans leucopsis

Olor cygnus columbianus Guara alba buccinator

Plegadis autumnalis Mycteria americana Botaurus lentiginosus Ixobrychus exilis Ardea herodias Herodias egretta Egretta candidissima Florida caerulea Butorides virescens Nycticorax nycticorax naerius Nyctanassa violacea

Grus americana canadensis mexicana

Rallus elegans crepitans virginianus

Porzana carolina

Coturnicops noveboracensis . Creciscus jamaicensis Crex crex Ionornis martinica

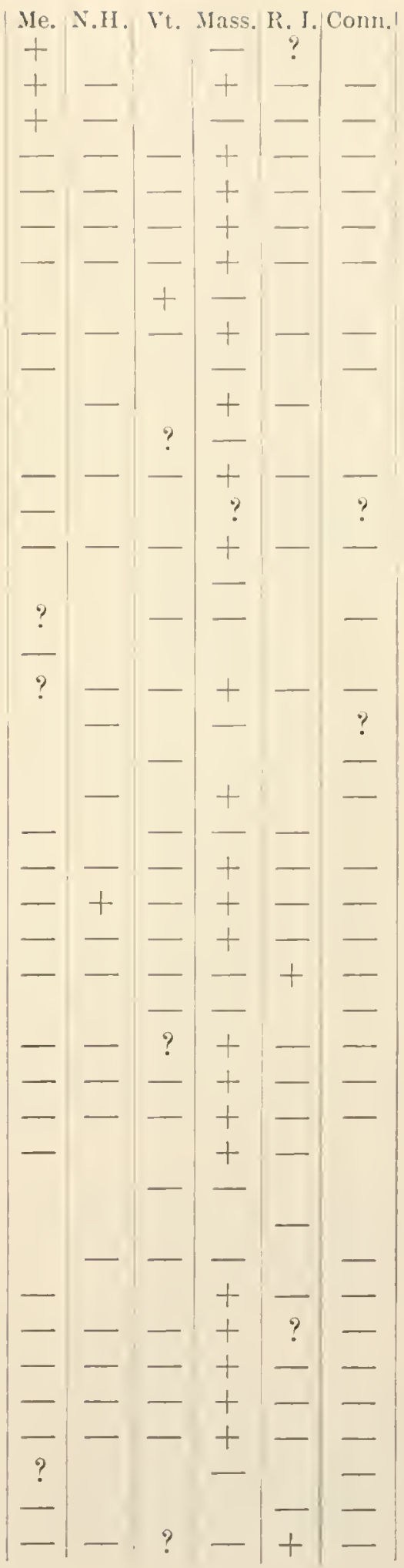


Gallinula galeata

Fulica americana

Phalaropus fulicarius

Lobipes lobatus

Steganopus tricolor

Reculrirostra ancicana

Himantopus mexicanus

Philohela minor

Gallinago delicata

Macrorhamphus griseus .

scolopaceus

Micropalama himantopus

Me. N.H. Vt. Mass. R. I. Com.

Tringa canutus

Arquatella maritima

Pisobia maculata

fuscicollis

bairdii

minutilla

Pelidna alpina

Erolia ferruginea

sakhalina.

Ereunetes pusillus mauri

Calidris leucophaea

Limosa fedoa haemastica

Totanus melanoleucus flavipes

Helodromas solitarius

Catoptrophorus semipalmatus . . .

Pavoncella pugnax

Bartramia longicauda

Tryngites subruficollis

Actitis macularia

Numenius americanus

hudsonicus

borealis

Squatarola squatarolir .

Chararlrius dominicus

Oxyechus rociferus

Aegialitis senipalmata meloda

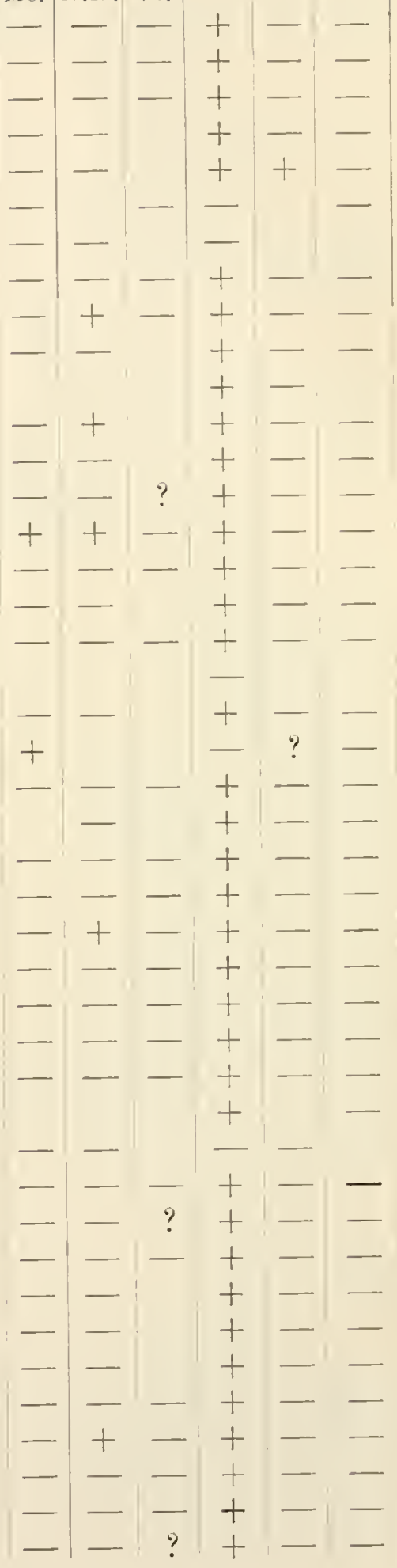


Ochthordromus wilsonius

Arenaria interpres

Haematopus palliatus

morinella

Colinus virginianus

Canalchites camadensis canace

Bonasa umbellus

Lagopus lagopus

togata

Trmpanuchus cupido

Mícleagris gallopavo silvestris

Ectopistes migratorius

Zenaidura macroura carolinensis

Starnocnas cyanocephala .

Cathartes aura septentrionalis.

Catharista urubu

Elanoides forficatus

Circus hudsonius

Accipiter velox

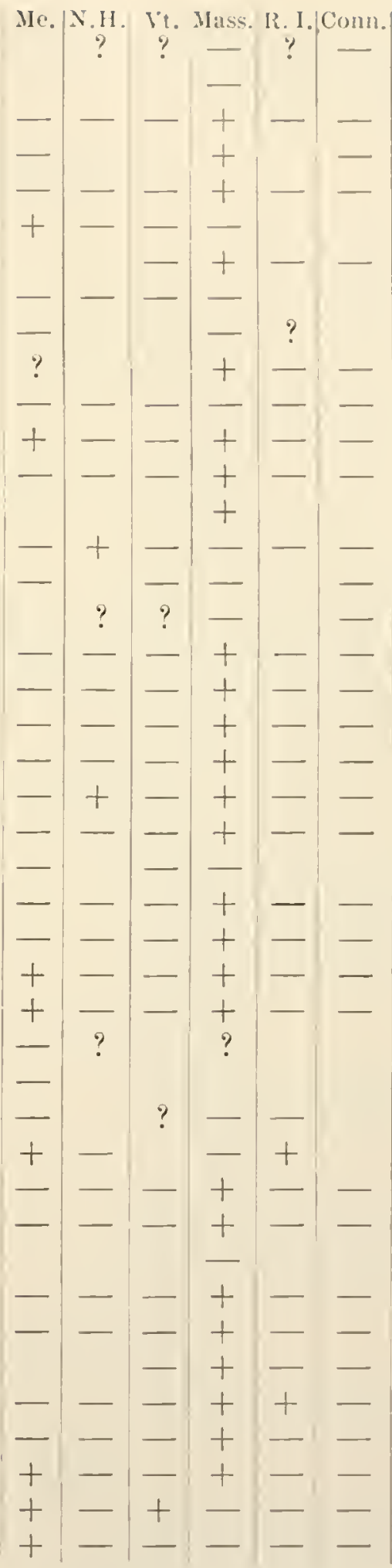


Glaux acadicus

Otus asio

Bubo virginianus

Nyctea nyctea

subarcticus

heterocnemis

Surnia ulula caparoch

Speotyto cunicularia hrpogaea

Coccyzus americauns erythrophthalmus .

Cervle alcron

Dryobates villosus.

Picoides arcticus

$$
\text { pubescens medianus }
$$
americanus

Sphrrapicus varius

Phlocotomus pileatus abicticola

Melanerpes ervthrocephalus

Centurus carolinus .

Colaptes auratus luteus

Antrostomus carolinensis . rociferus

Chordeiles virginianıs

Chaetura pelagica

Trochilus colubris

Muscirora forficata

Tyтamus tyramnus

$$
\text { dominicensis }
$$

rerticalis

Myiarchus crinitus

Sayornis phoebe

$$
\text { saya }
$$

Nuttallornis borealis

Myiochanes virens

Empidonax flaviventris

virescens.

trillin aliorim

Otocoris alpestris minimus.

praticola horti

Cranocitta cristata

Me. 'N.H.| Vt. Mass. R. I. Conn.

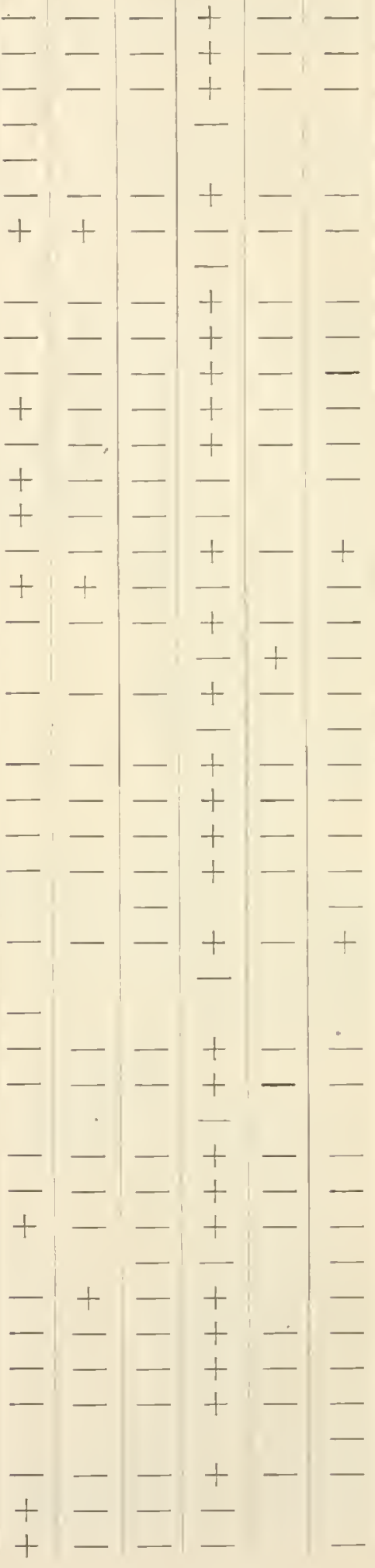

Corvus corax principalis 
Corrus brachyrhyuchos ossifrigus

Sturnus vilgaris

Dolichonyx oryzivorus

Molothrus ater

Xanthocephalus xanthocephalus

Agelaius phoenicens

Sturuclla migna fort is

Ieterus spurius gillbulir bullocki.

Euphagus carolimus

Quiscalus quiseulia.

Hesperiphona vespertina

Pinicola enudeator leucura

Carpodacus purpureus

Loxial curvirostra minor

$$
\text { lencoptera }
$$

Acanthis hornemannii exilipes linaria

Astragalinus tristis holboellii rostratil.

Spinus pinus

$$
\text { spinus }
$$

Plectrophenax nivalis .

Calcarms lapponicus

$$
\text { ornatus . }
$$

Pooecetes grimineus

Paisserculus prineeps

$$
\text { sandwichensis savanna }
$$

Coturniculus salvannarum australis Ammorlramus henslowii . caludaciutus nelsoni maritimus

subvirgatus

Chondestes grimmacus

Me. N.H. Vt. Mass. R. I. Conn.'

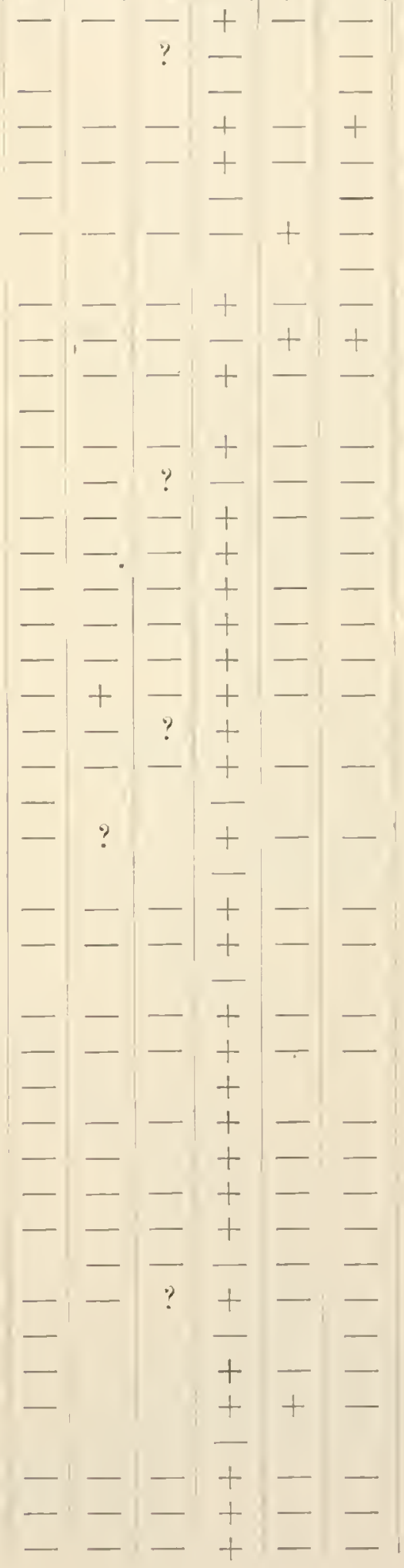

Spizella monticola . 
Spizella passerina breweri pusilla

Junco hyemalis

montanus

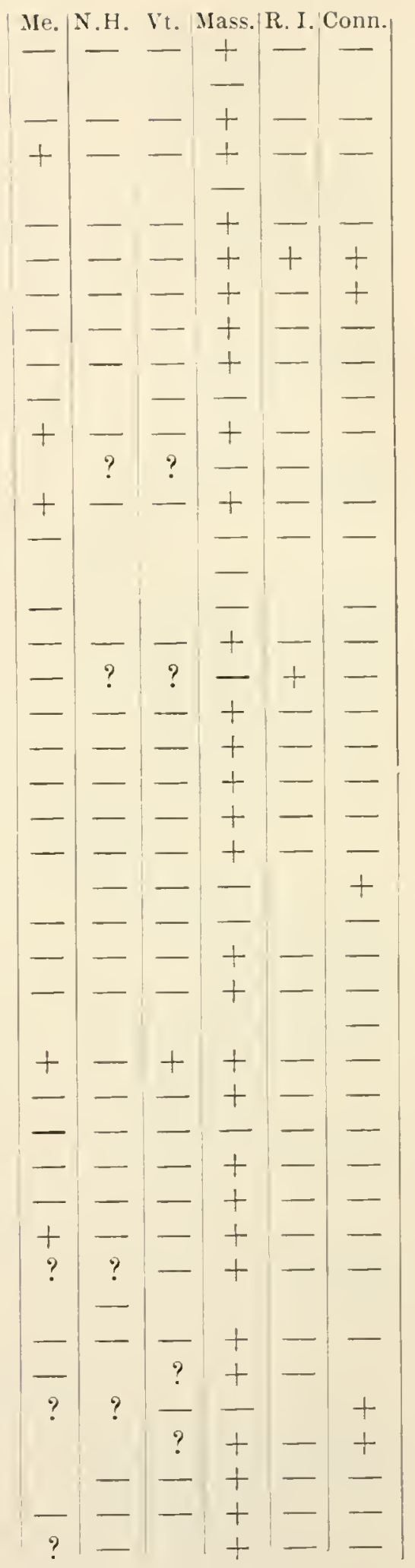

Passerella iliaca

Pipilo erythrophthalmus

Cardinatis cardinalis

Zamelodia hudoriciana

Guiraca caerulea

Passerina cyanea

Spiza americana

Calamospiza melanocorys

Piranga hudoviciana

Progne subis erythromelas rubra

Petrochelidon lunifrons

Hirundo erythrogaster

Iridoprocne bicolor

Riparia riparia

Stelgidopteryx serripennis

Bombycilla garrula

Lanius borealis cedrorum .

$$
\text { ludoricianus }
$$

Vireosylva olivacea migrans philadelphica gilva

Lanivireo flarifrons Vireo griseus . solitarius

Mniotilta varia

Protonotaria citrea

Helmitheros vermivorus

Helminthophila pinus

chrysopter

rubricapilla

celata 
Hedminthophilal peregrina

Compsothlypis anericana usneae.

Dendroical tigrina

alestiva

(atcrulescens

roronata

aurluboni

magnolia

rerulea .

pelistranica

striata

blackburniae

catstillea

dominiea

virens

vigorsii .

palmarum

discolor

hỵpochrysea

Seiurus aurocapillus

Sciurus noreboracensis

notabilis

motacilla

Oporornis formosa

agilis

philadelphia

Geothlypis trichas

Icteria virens

Wilsonia citrina.

pusilla .

canadensis

Setophaga ruticilla

Anthus rubescens

Mimus polyglottos.

Dumetella cir rolinensis

Toxostoma rufum

Thryothorus ludovicianus

'Thromanes bewickii.

Troglodytes aerlon.

Namums hiemalis

Cistothorus stellaris

'Telmatodytes palustris

Corthia familiaris americana

Sitta carolinensis
Me. N.II. Vt. Mass. R.I. Conn.

$+-1-$

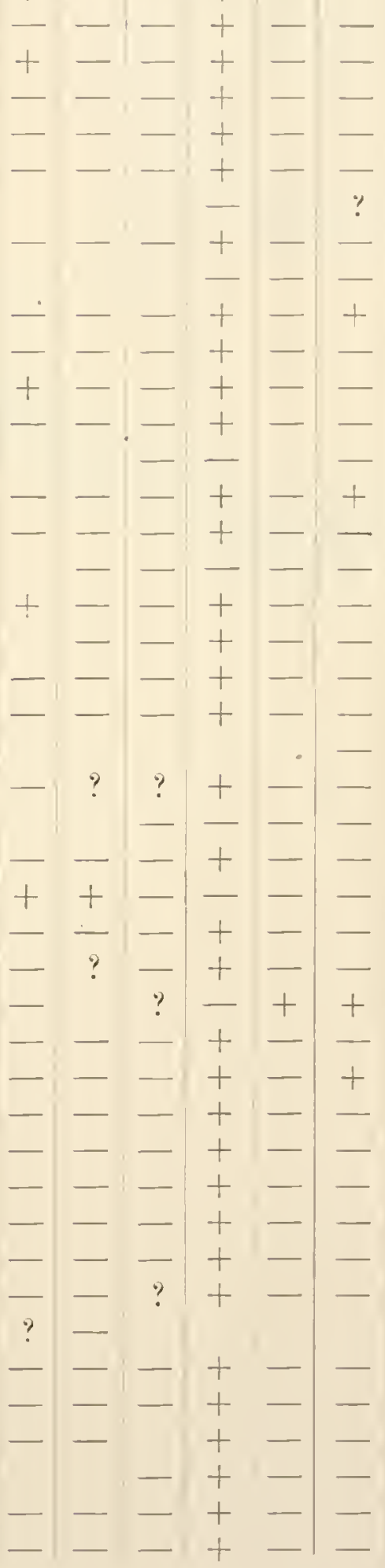


Sitta canadensis

Me. N.H. Vt. Mass. R. I. Conn.

Bateolopluts bicolor

Penthestes atricapillus

hudsonicus

Regulus satrapa

calendula .

Polioptila caerulea.

Hylocichla mustelinal

fuscescens

aliciae

salicicola .

bicknelli

ustulata swainsonii

guttata pallas

Planesticus migratorius

Ixoreus naevius

Sialia sialis 




\section{A V ES.}

PYGOPODES.

COLYMBI

COLYMBIDAE.

1. Comynes homoldull (Reinhardt) Ridgway.

Holboell's grebe; Red-necked grebe.

Podirepss holbüllii Reinh., Vidensk. meddel. naturh. forh. Kjöbenhaven for 185:3, 18.5t, p. 76. "Grönland . . JullanehaAlis DistrikT."

Podiceps rubricollis Lath. Autubon, Birds of Amer., 1844, vol. 7, p. 312 pl. 480.

Fresh and salt water; nests among rushes in sloughs.

Mr-Lncommon winter resilent coastwise. Sept.-April 24.

N. H.- Uncommon nigrant and coastwise winter resident. Oct.-May (June).

Vт.-Rare migrant: Middlebuly; Wells River; Windsor, spring.

MAss.- Uncommon migrant and winter resident, mainly coastwise. (Sept.) Oct. 10-May 24."

R. 1.- Incommon winter resident coastwise. Oct. 23-May 2.

Coxs.- Lncommon winter resident coastwise. Oct. 19-spring.

2. Colvanes auretes Limné.

Horned grebe; Devil diver; Hell diver; Little diver; Tinker; Tinker loon.

Limné, Syrst. nat., ed. 10, 1758, rol. 1, p. 135. "In summis Europat \& Anerica lacubes."

Podiceps rormutus Linné. Audubon, Birts of Amer., 1St4, rol. 7, p. 316, pl. 481. Egg, Capen, 18S6, pl. 24, fig. 3.

Salt and fresh water; nests anong rushes in marshes.

OCC. PAPEK H. …. 11. 1909. VOL. 
PYGOPODES.

COLTMBI.

COLYMBIDAE.

Me.-Common migrant, main!y coastwise; rare winter, and in northeastern part, rare summer resident.

N. H.- Lncommon migrant and winter resident, mainly coastwise. Oret.-April.

VT. - Rare migrant.

MAss.-Common migrant and winter resident, mainly coastwise. Oct. 1-. Lay 6 (June 1, Springfield).

R. I.-Common winter resident coastwise. Oct. 23-April 24 (Iay).

Coxx-Common winter resident coastwise; accidental in summer (Melrose, July 26, 1S\$2). Sept.-Mặ (July 26).

3. Podrlimes podicers (Limné) Lawrence.

Pied-billed grebe; Dabchick; Devil diver; Diedapper; Dipper; Frog-in-throat; Hell diver; Water witch.

Chapman, Handb. birts east. No. Amer., 1895, p. 57; Birdlife, 1898, pl. 2. Egg, Capen, 1Ss6, pl. 24, fig. j.

Colymbus podiceps Linné, Srst. nat., ed. 10, 175s, rol. 1, p. 136. "IN AMERICA SEITENTRIONALI."

Freshwater rivers, ponds, swamps; nests among reeds in swamps.

Me.-Common migrant, mainly coastrise; less common summer resident. April-May 19; summer; Sept. 1-Nor. 13 (late Nov.). Eggs, June 6.

N. H.- Uncommon migrant and rare summer resident. Mar.Nor.

VT.- Rare summer resident. Spring-Oct. 19.

MAss.- Rare spring, common fall migrant, and uncommon local summer resident; accidental in winter. (Feb.) Mar. 1-Defe 10. Eggs, April 23-June 2S.

R. I.- Unconmon migrant and summer resident. April-Oct. 22. Eggs, June 6 .

Coxx.-Common migrant, mainly in fall, and rare summer resident. April-summer; Sept--Nor. (Dec. 25, 1908, South Norwalk). 
PYGOPODES.

C'EPPHI.

GAVIIDAE.

4. Garia mamer (Brünnich) A. O. L. comm.

Loon; Big loon; Call-up-a-storm; Great northern diver.

Colymbns immer Brïnn., Ornith. borealis, 1764, p. 39. "I: FaERos."

Lrinator imber (Gunnerus). Chapman, Handb. birds east. No. Aner., 1S95, p. 5s; Bird-life, 1S9S, pl. 3. Fgg, Capen, 18S6, pl. 24. fig. 2 .

Salt and fresh water; nests on shores of lakes.

ME., X. H.-Common migrant, less common winter and summer resident. Eggs, June 11-19 (July).

$\mathrm{r}_{\mathrm{T}}$ - L Lncommon summer resident.

Mass.-Common migrant and winter resident, mainly coastwise; rare summer resident inland (formerly Springficld); a few non-breeding birds summor oftshore.

R. I.-Common migrant and winter resident coastwise; a few non-breeding birels summer offshore. Sept. 15-May 2S; smmmer.

Cons.-Common winter resident coastwise; formerly a rare summer resident (Easthampton). Oct.-April 29.

5. Gava arotica (Linné) Allen.

\section{Black-throated loon or diver.}

C'olymbus aretiens Iinné, Sist. nat., ed. 10, 1758, vol. 1, p. 135. "Ix Europa \& America IBOrFan." Audubon, Birds of Amer., 1844, rol. 7, p. 295, pl. 477. F,gg, Seebohm. 1S96, p. 7S, pl. 21, fig. 1 .

Seacoasts; nests on the ground.

ME.-Rare winter visitor: ('lina, Kennebec Co.. April 19, 1899 ; Cutler, Dee. S, 1 SS1.

Mass. - I oubtfully recorded.

Coxs:- Doubufully recorded (? near Southbridge, Mass.). 
PYGOPODES.

CEPPHI.

GAVIIDAE

6. Gaila steldata (Pontoppidan) A. O. L. comm.

\section{Red-throated loon; Cape-race; Little loon; Red-throated diver; Scape-grace.}

Colymbus stellatus Pontopp., Danske atlas, 1763, vol. 1, p. 621. (Based on Villughby.)

Colymbus septentrionalis Linné. Audubon, Birds of Amer., 1844, vol. 7, p. 299, pl. 47S. Egg, Seebohm, 1S96, p. 78, pl. 21, fig. 2.

Salt water mainly, sometimes on lakes; nests on the ground.

ME., N. H.-Common migrant and winter resident, mainly coastwise. (Aug. 16) Sept. 22-(June 22).

Vt - Rare migrant. Nov. 1.

Mass. - Uneommon spring, common fall migrant and winter resident, mainly coastwise; casual in summer. Ang. 27-April 8 (July 2).

R. I.-Common migrant and winter resident coastwise; a few non-breeding birds summer offishore. Oct.1-April 27 (summer).

Coxy.-Common winter resident, coastwise. Oct.-May.

\section{ALCIDAE.}

7. Luxda cirrhata (Pallas) Pallas.

Tufted puffin.

Alea circhata Pallas, Spic. zool., 1769, fasc. 5, p. 7, pl. 1; pl. 2, figs. 1-3. "in Mari inter Kamtschatkan et Americali Archipelaguage Kurilum."

Mormon cirrhatus Lath. Andubon, Birds of Amer., 184t, vol. 7, p. 234 , pl. 462 .

Rocky sea coasts; nests in burrows.

Me.-Aceidental visitor: month of Kennebec River, winter of $1831-32$. 
PIGOPODES.

S. Fraterclia arctica (Limné) Schä̈llor.

\section{Puffin; Paroqueet; Sea parrot.}

Alca arctica Linné, Ș̣st. nat., cd. 10, 1758, vol. 1, p. 130. "1N Ecropate borealis ockano."

Mormon arcticus Limné. Audubon, Birds of Amer., 18tt, vol. 7, p. 238, pl. 464. Egg, Capen, 1856, pl. 24, fig. 6.

Rocky seacoasts; nests in burrows.

ME.-Common winter resident coastwise, and rare summer resident (Matinicus Rock and Machias Seal Island).

N. H.- Uncommon winter visitor (at Isles of Shoals).

MAss. - Uncommon winter visitor coastwise. Oct. 16-Marreh.

R. I.- Doubtfully reported.

9. Cepretes grrlate (Limné) Brehm.

\section{Black guillemot; Pigeon murre; Sea pigeon.}

Alca grylle Linné, Sịt. nat., edl. 10, 175\$, rol. 1, p. 130. “Ix EUROPAE BOREALIS OCEANO."

Uria grylle (Linné). Auduhon, Birds of Amer., 1stt, rol. T. p. 272, pl. 474. Eggg, Capen, 1S86, pl. 24, figs. 7, S.

Rocky seacoasts; nests in deep crevices of rocks.

ME.-Common winter resident coastwise; less common summer resident from Knox Co. castwind. Egrgs, June 15-23.

N. H.- Uncommon winter visitor coastwise.

Mass.- Encommon winter risitor coastwise. Sept.-April 19 (May).

R. I.- Rare winter visitor. Nor. 18-April.

Coxs- Rare winter visitor roastwise: Stony Creek, Dec., $15 S T$. 
PYGOPODES.

CEPPHI.

ALCII)AE.

10. Uria tronle (Linné) Latham.

Murre; Foolish guillemot; Guillemot.

Cones, Key No. Amer. birds, ed. 5, 1903, vol. 2, p. 1082, figs. 742,743 .

Colymbus troille (sie) Linné, Fauna Suec., exl. 2, 1761, p. 52. "IN ALTO MARI SETTENTRIONALI."

Alca troile (Limé). Dresser, Birds of Europe, 1871-81, vol. \$, p]. [621]. Eggg, Capen, 1886, pl. 24, fig. 9; pl. 25, fig. 1-3.

Rocky seacoasts; nests on sea cliffs.

Mr.-Rare winter risitor: Eastport, Dec. 27, 18s6; Scarborough.

VT.-Doubtfully recorded.

Mass.- Rare winter visitor: "Massachusetts" (Society collection).

R. I.- Doubtfully recorded.

Cons.-? Rare winter visitor (Merriam, probably in errol for U. lomvia).

11. Uria lomvia (Limné) Bryant.

Brünnich's murre; Brünnich's guillemot; Ice-bird; Large-billed guillemot; Sea crow.

Alca lomvia Linné, Syrst. nat., ed. 10, 1758, rol. 1, p. 130. "IN Eurota boreali."

Uria brumichii Sabine. Audubon, Birds of Amer., 18t4, rol. 7, p. 265, pl. 472. Egg, Seebohm, 1896, p. 94, pl. 26, figs. 5, 6.

Rocky seacoasts; nests on sea cliffs.

Me.-Common winter risitor coastwise, casual inland; a doubtful breeding record (Penobscot Bay, M. Hardy). Nor. 23-Mar.

N. H.- Irregular winter risitor coastwise; occasional inland. Nov. 25-Mar. 
PYGOPODES.

C'EPPH.

ALCIDAE.

V'T.-Irregular winter visitor. Nor.-Jan.

Mass.- Turommon, irregular winter visitor coastwise; oecasional inland. Nov. 21-Mar. 14 (April 15).

R. I.- Encommon and irregular minter visitor. Nor. 15Feb. 23.

Coxy.- Irregular winter visitor, sometinnes common. Nor. 18Fob. 16.

\section{Arca tomd Iinné.}

\section{Razor-billed auk; Razor-bill; Tinker murre; Turre.}

Limé, Syst. nat., ed. 10, 1758, vol. 1, p. 130. "IN FuropaE BOrkals oceano." Andubon, Birds of Amer., 184t, rol. 7, p. 247, pl. 466. Egg, Capen, 1886, pl. 25, fig. 4-6.

Rocky seacoasts; nests in rock creviees.

Me.-Common winter visitor coastrise; said to have nested formerly.

N. H.- Uncommon winter risitor coastwise. Nor--NIar.

MAss.- Uneommon winter visitor coastwise. Nor. 1-April 15.

R. I.- Rare winter visitor coastwise. Nov. 15-Fob. 4.

Cons. - Ralle minter visitor constwise.

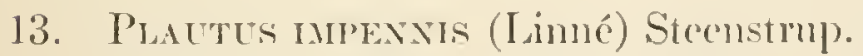

\section{Great auk; Garefowl.}

Alca impenmis I inné, Syst. nat., ed. 10, 175S, rol. 1, p. 130. "IN Eurola ARCTica." Audubon, Bircls of Amer., 1St4, rol. 7, p. 245, pl. 465. Egg, S. Griere, Great Ank, or Garctowl, L _ondon, 1SS5, pls. 1, 2 (opp) pl). 10S, 109).

Formerly a coastwise species, breeding at Funk ld., near Xerwfomdland, and at a fer places on the European coast; last specinnen killed in $184 t$. 
PYGOPODES.

CEPPHI.

ALCIDAE.

Me., N. H., Mass.- Formerly a winter visitor coastwise.

R. I., Coxw.- Probably oceurred formerly ats a winter risitor coastwise (bones in shell heaps at Block Id., R. I.).

14. Alle alle (Limné) Stejneger.

Dovekie; Ice-bird; Knotty; Little auk; Pine-knotty; Rotche; Sea dove.

Alca alle Linné, Sysst. nat., ed. 10, 175S, vol. 1, P. 131. "Ix Europae, Anericae arcticae oceano."

Mergulus alle Limné. Audubon, Birds of Amer., 1844, vol. 7, p. 257, pl. 469. Egg, Seebohm, 1896, p. 95, pl. 26, fig. 3.

Rocky coasts; nests on ledges or in crerices of sea cliffs.

ME.- Uncommon winter risitor coastwise, easual inland; Late Nov.-Mar. 15.

N. H.- Uncommon winter visitor coatstwise; calsual inland.

TT.-Accidental winter visitor.

MAss- Uncommon winter visitor, coastwise; casual inland. (Sept.) Nov. 4-May 1.

R. I.- Rare and irregular winter visitor coustwise; casual inland. Nor. 1t-April 27.

Cons.- Rare and irregular winter visitor coastwise; casual inland. (Sept.) Nov. 10.

LONGIPENNES.

STERCORARIIDAE.

15. Megalestris skta (Brünnich) Ridgway.

Skua; Great Skua; Sea-hen.

Catharaeta slua Brünn., Omith. borealis, 1764, p. 33. "E Feroa \& Islandia."

Megalestrix catarhactes (Limné). Sannders, Cat. birds British mus., 1896, vol. 25, p. 315. 
LONGIPENNES.

\author{
STERCORARIIDAE.
}

Stercorarins catarhactes (Limné). l)resser, Birds of Europe, 1S71-81, vol. \&, pl. [609]. F.gg, Sechohın, 1896, p. 118, pl. 37, figs. 3,4 .

Pelagie; nests on rocky cliffis or tundrals.

Mass-Rare risitor offshore: Georges Banks, July, 187S; Nantucket Shoals, Oct. 11, 1883; Polluck Rip, Sept. 10, 1884.

16. Stercoraries pomarines ('T'mminck) Vieillot.

\title{
Pomarine jaeger.
}

Lestris pomarinus 'T'emm., Min. d'ornith., 1S15, p. 514. “Les REgioxs du CERCle AnCTique." Audubon, Birds of Amer., 184t, vol. 7 , p. 186, pl. 451. Egg, Secbohm, 1896, p. 116, pl. 37, fig. 6.

Largely marine; nests on the gromel.

Me.- Uncommon migrant, casual in summer. Mary; (June 23, 24); July 14-late Oct.

MAss.- [ncommon migrant, occasional in summer. May 23; July j-Sept. 28.

R. I.- Cncommon migrant oftshore, mainly in fill. Oct.

17. Stercoraries liarasiticts (Limué) Schioffer.

\section{Parasitic jaeger; Gull chaser; Marlinspike; Richardson's jaeger; Robber gull; Sea hen.}

Lanns parasitions Limné, Syst. nat., erl. 10, 1758, vol. 1, p. 136. "intra tropicum Cancri, Euroral, Americae, Asiak."

Lestris richardsomii Swains. Audubon, Birds of Amer., 1S4t, vol. 7, p. 190, pl. 452). Eggg, Secbohm, IS96, p. 116, pl. 37, figs. 1, 2. Largely maluine; nests on the ground.

ME.- Encommon migrant offshore; casual in stmmer. MalyJune; (July) Aug.-Oct. 
LONGIPENNES.

STERCORARIIDAE.

N. H.- Uneommon migrant offshore. Sept.

MAss. - Uncommon migrant and sumner visitor offshore; rare in winter. May 31-Oct. 14 (Jan., Feb.).

R. I.- Rare migrant oftshore: Charlestown Beach, three, Sept. 2, 1597 .

Conv.- Rare migrant offshore: Bridgeport; Portlind, fall, 1875.

19. Stercoraries longicaudus Vieillot.

Long-tailed jaeger; Buffon's jaeger or skua; Marlinspike; Whiptail.

Vieill., Nouv. dict. d'hist. nat., 1819, vol. 32, p. 157. "LE NORD de l'Europe, de l'Asie et de l'A Aérique."

Lestris parasiticus Linné. Audubon, Birds of Amer., 1S44, vol. 7, p. 192, pl. 453. Egge, Seebohm, 1896, p. 117, pl. 37, fig. 5.

Largely marine; nests on the ground.

ME.- Rare migrant offshore.

VT.-Accidental migrant: West Castleton, Sept. 7, 1877.

MAss. - Rare migrant. Spring; Aug. 24-Sept. 29.

Conn.- Rare or accidental visitor: Community Lake, Wallingford, Aug. 30, 1873.

\section{LARIDAE.}

19. Pagorhila alba (Gunnerus) Cones.

\section{Ivory gull.}

Lamus albus Gunn., in Leem's Beskriv. Finmark. Lapp., 1767, p. 28.5 (note). Northern Norway.

Larus eburneus Gmel. Audubon, Birds of Amer., 1St4, vol. 7 p. 150, pl. 445. Eggg, Seebohm, 1896, p. 115, pl. 32, fig. 3.

Arctic seas, following the pack ice; nests on rocky clifts. 
LONGIPENNES.

\section{I.ARIDAE.}

ME.- The Boardman record refers to a New Brunswick specimen.

Mass.-Accirlental winter visitor: Monomoy Id., I)ec. 1, $18 S 6$.

20. Risat TRIDACTTha (Linné) Bonaparte.

Kittiwake; Frost-bird; Jack gull; Meterick; Pinny owl or Pinyole; Snow gull; Winter gull.

Lams tridactylus Limné, Syrst. nat., ed. 10, 1758 , vol. 1, p. 136. "is Eirropa septentrionali." Audubon, Birds of Amer., 18t4, rol. 7, p. 146, pl. 44t. Egg, Sammels, Ornith. and ool. N. E., 1867 , pl. 4, fig. 3.

Inritime; nests on sea clifts.

ME.-Common fall and winter visitor offishore; casual inland, and in summer. Nor.-April (Junc; July 11, 1903, Jericho Bay, barren bird; July 14, 1907, off Portland).

N. H.-Common fall and winter risitor offishore. Tor. 1spring.

VT.- Rare winter visitor: Enosburg Falls, Nov. 12, 1906; St. Albans; St. Johnsbury.

MAss.-Common fall and winter visitor offshore, casual inland. Sept. 6-Mar. 10.

R. I.- Common fall and winter visitor offishore. Nor.-Mar. 23.

Coxs.- Uncommon fall and winter visitor offishore. FallApril 14.

21. LARCS HYTERBorets Gumnerus.

Glaucous gull; Burgomaster; Owl gull; White minister.

Gumnerus, in Lecm's Beskriv. Finmark. Lapp., 1767, p. 226 (note), p. $28: 3$ (note). No locality.

Larus glaucus Brünn. Audubon, Birds of Amer., 18t4, vol. T, p. 170, pl. 449. Egg, Secbolım, 1S96, p. 112, pl. 32, figs. 4. 5.

Seacoasts; nests on clifl's. 
LONGIPENNES.

\section{LARIDAE.}

ME. - Rare winter risitor. Iate Nov-April 27.

․ H.- Rare winter risitor: Hampton, about lieb. 20, 18S6, and in Mar.

VT.- Doubtfully recorded.

Mass.- Rare winter visitor. Nor. 29-April 23 (May 26, 1907, Ipswich).

R. I.- Rare winter visitor: Newport, Mar. 12, 1900 (three): Dec. 1901.

\section{IARL'S IELCOPTERI'S Fabel.}

\section{Iceland gull; White-winged gull.}

Faber, Prodr. Isl. ornith.; 1822, p. 91. "Im Mixter.....АсH Istaxd." Saunders, Cat. birds British mus., 1896, rol. 25, p. 296. Audubon, Birds of Amer., 1844, vol. 7 , pl. 4t7. Egg, Seebohm 1896, p. 113, pl. 33, fig. 3 .

Seacoasts; nests on the ground.

Me.- Rare winter risitor. Nor.-April.

Mass- Rare winter visitor. Nor. 4-Mar. 20.

Coxx- - Rare winter visitor: Stamford, Feb. 16, 1894.

\section{JaRUs KUMIIENI Brewster.}

\section{Kumlien's gull; Lesser glaucous-winged gull.}

Brerst., Bull. Nuttall ornith. (Jub), 1883, rol. 8, p. 216. "C'cuBerlain Solvo, Arctic Anerica." Dwight, Auk, 1906, rol. 23, p. $36+1$, pl. 1, figs.

Coastwise; breeds on Arctic coasts.

Me. - Probably occasional winter visitor: near Eastport (nay have been N. B.); "seen" in Portland Harbor" and at Scarborough.

MAss.- Rare winter visitor: Boston Harbor, Feb. 22, 1905; 
LONGIPENNES.

H.ARTD.AE.

Plymouth, Jan. 5, 1SSS (1)wight, immatmre); Boston and Swampscott, two or more, Dec. 29, 1907-Mar. 13, 1908.

Coxs.- Rare winter visitor: Stamford, Feb. 16, 1894 (1)wight, immature).

24. IARTS MARINLE Iinné.

Great black-backed gull; Black-back; Black minister; Coffin carrier; Ghost-bird; Minister; Saddle-back.

Limé, Sịst. nat., ed. 10, 175S, rol. 1, p. 136. "Ix Europ.." Audubon, Birds of Amer., 184t, vol. 7, p. 17.2, pl. 450. Egg, Capen, 1S86, pl. 22, figs. 2, 3.

Seacoasts; nests on the ground.

ME.-Common migrant and winter resident; casual summer visitor. Sept. 21-April (summer).

N. H.-Common winter resident coastwise. Oct. 15-spring.

Mass. Common winter resident coistwise; a few non-breceling birds summer. I alte Aug.-May 1 (summer).

R. I.- Common winter resident coastwise. Ang. 26-April 23.

Coxx.- Uncommon winter resident coastwise. Fall-Mall.

25. Lares argentates Pontoppidan.

Herring gull; Gray gull; Sea gull; Winter gull.

Pontopp., Danske atlas, 17633, rol. 1, p. 622. No locality. Audubon, Birds of Amer., 1St4, vol. 7, p. 163, pl. 4ts. Eigg, Capen, 1S86, pl. 22, fier. 4-6.

Seacoasts, large rivers and lakes; nests on the ground or rallely in trees.

ME.- Abundant resident, breeding mainly on outer islands, rarcly on inland lakes. Eggs, (May 14) June 12-July 18 (Aug. 19). 
LONGIPENNES.

\section{LARIDAE.}

․ H.-Common nigrant and winter resident; less common summer visitor coastwise.

VT.- Encommon migrint and rare summer resident (Lake (hamplain).

MAss - Common migrant and winter resident, less common summer visitor coastwise; not now known to breed (nested at Wepecket Id., 18s2, Mackay).

R. I.-Common migrant and winter resident, mainly coastwise; " few non-breeding birds summer. Sept. 10-May 22; summer.

Coxs.-Common migrant and winter resident, mainly coastwise; a few non-breeding birds summer. Oct.-May; summer.

\section{Lares delawarexsis Ord.}

\section{Ring-billed gull.}

Ord, Gutlurie's Geogr., hist., and commercial grammar, 2d Amer. erl., 1815, rol. 2, p. 319. "ox the Delaware below Phildoelipha." Chapman, Handb. birds east. No. Amer., 1895, p. 73.

Larus zonorhyuchuss Richarrlson. Audubon, Birds of Amer., $18+4$, vol. 7, p. 152 , pl. 446 .

Seacoasts; nests on the ground.

ME., N. H.- Uncommon migrant and rare winter resident coastwise. Mar.-April; Oct.-Nor: (winter).

MAss- - Incommon migrant coastwise. Spring; July 17Oct. 30 .

R. I.- Encommon migrant and winter resident. Nor:-April.

Coxx.- Lncommon migrant and winter resident. 
LONGIPENNES.

LARIDAE.

27. I.art's atrichat Iinné.

Laughing gull; Black-headed gull; Black-poll.

Limné, Syrst. nat., el. 10, 175s, rol. 1, p. 136. "America." Audubon, Birds of Amer., 1St4, rol. 7, p. 136, pl. 443. Egg, Capen, 1SS6, pl. 23, fig. 1-3.

Seacoasts; nests on the ground.

Me.- Rare summer resident (Metinic Green lel., Western Egg Rock). (Late April)-Sept. Eggos, Jume 30-July j.

VT.- Rare visitor: Bristol, May, 1SS3; St. Alhans.

MAss.- Uncommon summer resielent south of Cape Cod (MIsskeget Id.; formerly at Egge Rock. Nabant); elsewhere a rare visitos. May 7-Oct. 1 (late Yor.). Egegs, June 7-July 10.

R. I.- Rare summer visitor: Seakonnet Point, Sept., 1SS4; Seakonnet Point, Aug. 24, 1903.

Coxs.- Rare summer risitor; may breed rarely.

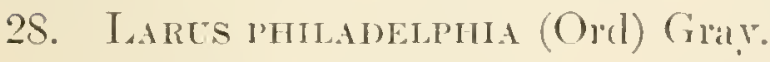

\section{Bonaparte's gull.}

Sterna plitadelphia Orel, Guthries's Geogr., hist., and rommercoll grammar, 2d Amer. ed., 1S15, vol. 2, p. 319. No locality; probaloly Philatiderhia.

Larms bonapartii Richardson. Audubon, Birels of Amer., 1Stt, rol. 7, p. 131, pl. H2. Egge. Secbohm, 1896, p. 106, pl. 36, fig. 7.

Salt and fresh water; nests on stumpes or in low trees.

Me.-Common migrant, rare in winter. April-.June 20 ; .July 25-Nor. 20 (winter).

X. H.-Common mignant and rare winter resident coastwise. May,; Ang. 3-Oct. 20 (winter).

VT.- Rale migrant; ?"smmmer resident."

MAss.-Common migrant, mainly constwise, and rare winter resident. Mal. 10-June 3; July 24-Xor. 25) (winter). 
LONGIPENNES.

\section{LARIDAE.}

R. I.-Common migrant and uneommon winter resident. Aug. $5-M a y 22$.

Coxx-Common migrant, mainly in fall. Aug. 1-Xov.; (winter ?)-April 21.

29. Xeala sabinil (Sabine) Leach.

Sabine's gull; Fork-tailed gull.

Larus sabinii J. Sab., 'Trans. Linn. soc. London, 181S, vol. 12, p. 520, pl. 29. "West COAst of Greenland . . L Latitude $75^{\circ}$ $29^{\prime}$ N., AND loxgitude $60^{\circ} 9^{\prime} \mathrm{W}$." Audubon, Birls of Amer., 1844, vol. 7, p. 127, pl. 441. Egg, Seebohm, 1896, p. 105, pl. 36, fig. 8 .

Arctic seas and coasts; nests on the gromnd.

Me.-Accidental visitor: Brothers Id., near Portland, Sept. 22, 1899; Scarborough, May 31, 1877; (nec Calais, 1878).

Mass.-Accidental visitor: Boston Harbor, Sept. 27, 1874; Cape Cod, 1Ss8; North Truro, Ang. 21, 1889.

R. I.- Doubtfully recorderl.

30. Gelochemdon xhotica (Hasselquist) Stejneger.

\section{Gull-billed tern; Marsh tern.}

Sterna nilotica IIasselq., Reise nach Pal., deutseh. ausg., 1762, p. 325. [Nile River, Egrpt].

Sterna anglica Montagu. Audubon, Birds of Amer., 1S4t, vol. 7. p. 81, pl. 430. Egg, Secbohm, 1896, p. 99, pl. 31, figs. 6, S.

Seacoalsts; nests on the ground.

ME.-Accidental risitor, several instances. March; May 21; Sept.

Mass.- Arcidental visitor: Ipswich, Sept. 1871.

R. I.- Doubtfully recorder. 
LONGIPENNES.

LARIDAE.

31. Sterna caspia Pallas.

\section{Caspian tern; Squawker.}

Pallas, Nov. comm. petrop., 1770, vol. 14, pt. 1, p. 5\$2, pl. 22, fig. 2. "alare caspilat et circa ostuca Iaici." Dresser, Birds of Emrope, 1S71-\$1, vol. S, pl. [5St]. Egg, Seebohm, 1S96, p. 100, pl. 31, fig. 2.

Sterna tschegrava Lepech. Chapman, Handb. birds east. No. Amer., 1895, p. 79, fig. 61, a.

Seareonsts and lakes; nests on the gromud.

Mr.- Rare migrant. May 11; Aug.-Sept. (Oct. 16).

N. H.- Rare migrant: Hampton, 1899.

Mass.- Rare spring and uncommon fall migrant. Early May; Aug. S-Sept. 26.

R. I.- Rare migrant. May 10; July 27-Oct.

32. Sterna maxima Boddaert.

Royal tern; Cayenne tern.

Bodd., 'Tabl. d. planch. enlum. d'hist. nat., 1783, p. 5S. Based on Buffon: "CAvevie." Chapmanr, Handb. birds east. No. Amer., 1595, p. 79, fig. 61, b.

Sterna cayana Lath. Audubon, Birds of Amer., 1St4, rol. 7 , p. 76, pl. 429.

Sealcoasts and lagoons; nests on the ground.

Mass.-Accidental visitor: Chatham, July 29, 1S89; Ipswich, July 17, 1904; Nantucket, July 1, 1874.

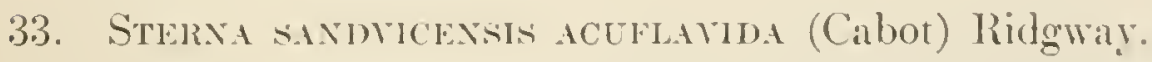

Cabot's tern.

Sterna acuflavida Cabot, Proc. Boston soc. nat. hist., $18+\bar{i}$, vol. 2, p. 257. "Taxcair, on tue Coast of Ícatan." 
LONGIPENNES.

\section{LARIDAE.}

Sterna cantiaca Gmel. Audubon, Birds of Amer., 1844, vol. 7 , p. 87, pl. 431.

Seacoasts; nests on the ground.

Mass.-Accidental risitor: Chatham, Aug.. 1865; Monomoy Id., Oct. 2, 1888.

34. Sterna forsteri Nuttall.

\section{Forster's tern.}

Nutt., Manual ornith. U. S. and Canada, 1834, rol. 2, p. 274. Based on Forster: "Churchill River," Hudsox Bay. Saunders, Cat. birds British mus., 1896, vol. 25, p. 47.

Sterna havellii Audubon, Birds of Amer., 1S4t, vol. T. p. 103, pl. 434 .

Marshes and sea coasts; nests in grassy marshes.

Mass.- Rare fall migraut. Aug.-Oct. 2.

35. Sterna hirutido Linné.

Common tern; Mackerel gull; Medrake or Medrick; Redshank; Sea swallow; Summer gull; Wilson's tern.

Limé, Srst. nat., ed. 10, 175S, vol. 1, p. 137. "Europ'." Chapman, Handb. birds east. No. Amer., 1595, p. S1, fig. 61, c; Audubon, Birds of Amer., 1St4, vol. 7 , p. 97, pl. 433. Egg, Capen, 1SS6, pl. 23, figs. 4,5 .

Seacoasts; nests on sandy or pebbly shores.

Me.-Abundant summer resident coastwise. May 3-Sept. 2 (late Sept.). Eggs, late May-Aug. 19.

N. H.- Common migrant coastwise, casual inland; formerly summer resident at Isles of Shoals. May; Aug.-Sept.

V't.-Rare risitor: Bennington, Мay 30, 1907; St. Albans. 
LONGIPENNES.

$$
\text { LARIDAE. }
$$

Mass- Locally common summer lesident coastwise, casual inland; accidental in winter (Woods Hole, Jan. 17, 1S94, Feb. 20, 1891). May 1-O(•t. 30 (Xor. 14, winter). Fgass, May 20July 30 .

R. I.-Common summer resielent coastwise. May 10-Oct. 6. Eggs, June 5-July 11.

Coxx-Common local summer resident coastwise. Eggs, June 20.

36. STERAA PARADISAEA Briminich.

\section{Arctic tern.}

Brïnn., Ornith. borealis, 1764, p. 46. "Christansör," I)EnMARK. Chapman, Handb. birds east. No. Amer., 1S95, p. S2, fig. $61, d$.

Sterna aretica 'Temm. Aulubon, Birds of Amer'., 1SH4, rol. T, p. 107, pl. 436. Egg, Capen, 1856, pl. 23, fig. 6-S.

Scacoasts; nests on the ground.

ME.-Common summer resident coastwise. May-Sept. Eggos, June $2 S$.

Mass.- Encommon local stmmer resident coastwise; occasional inland. Mary-Or.t.

Conx.- Migrant.

37. STERNA DOLGald. Montagn.

\section{Roseate tern; Mackerel gull.}

Montagu, Oruith. dict., suppl., 1S13, p. [3S6], 1 p]. "west Highlandos or Scotraxt," ('hapman, Ilandb. birds cast. No. Amer., 1S95, p. S3, fig. 61, c; I)resser, Birels of Europe, 1S71-\$1, vol. S, pl. [5S1]. Egg, ('apen, 1SS6, pl. 23, fig. 9-11.

Seacoasts; nests on sindy or pebbly shores. 
LONGIPENNES.

\section{LARID.AE.}

Me. - Rare summer visitor coastwise; not certanly known to breed. May-Aug. 31.

N. H.- Formerly rare summer resident at Isles of Shoals.

MAss. - Locally common summer resident coastwise. May 1Oct. t. Eggs, May 20-July 30 .

R. I.-Common migrant and summer visitor; not known to nest. May-Oct.

Coxi-Common migrant and local summer resident. Eggs, June 20.

38. Sterat antillarun (Lesson) Coues.

\section{Least tern; Little striker; Oyt.}

Stermula antillarum I.ess., Descr. mamm. et. ois., 1S47, p. 256. "Antilles... principalement Guadeloupe."

Sterna minuta Linné. Audubon, Birls of Amer., 1S4t, vol. T, p. 119, pl. 439. Egg, Capen, 1SS6, pl. 23, fig. 12-14.

Seaeoasts and lakes; nests on the ground.

ME.- Formerly rare summer resident in Casco Bay.

N. H.- Rare migrant: Hampton.

MAss. - Encommon summer resident, from Cape Cod sonth (formerly at Ipswich); elsewhere now casual. (April 27) May 4 Oct. Eggs, May 31-June 29.

R. I.- Now rare but formerly a more common summer visitor coustwise. -Ang. 20.

Coxx.- Rare migrant and summer visitor, formerly eommon.

-9. Sterata mocata Linné.

\section{Sooty tern; Egg-bird.}

Iinné, Srst. nat., ell. 12, 1766, vol. 1, p. 22S. "Is Irsula DoMinicexsi." 
LONGIPENNES.

\section{IAIRIDAE.}

Sterma fuliginosa I attl. Audubon, Birds of Aner., 1S4t, rol. T, p. 90, pl. 432. Egge, Sorbolin, 1S96, p. 104, pl. 30, fig. 4-6.

Scalcoatsts; nests on sandy sholes.

ME.- Aecidental visitor: Parkman, Oct. 5, 1S7s.

N. H.-Acedental visitor: Newmarlet, about Sept. 14, 18TS; (ner I alke Winncpesaukere).

Vt- Aceidental visitor: Ialke Champlain, early" Sept. 1876; Rutland, 1876 .

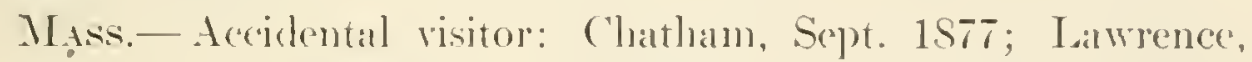
Oct. 29, 1876; Willimustown, Sept. 1S76.

R. I.- Accidental visitor: (") Newport, 1875; Point Judith, Sept. 1S76; Woonsocket, July 16, 1S97.

Coxx-Acidental visitor. Sept.

40. Hydrochelmon ngra strinamexsis (Gmelin) Strjugeger.

\section{Black tern.}

Stema sminamensis Ginel., Syst. nat., 17SS, rol. 1, pt. 2, p. 604. "SIRINA.I."

Stema migra Limné. Audubon, Birels of Amer., 1St4, rol. T, p. 116, pl. 438.

Fresh and salt water; mests in fresh marshes.

ME.- Rame fall miglant. Aug. 1t-2S.

N. H.- Cucommon migrant, mainly coastwise. June 10; Alig. $2+1-s(p)$ t. 30.

VT.- Ratre migrant: Latke Bomoseren, June ( $\mathrm{j}, 1853$.

Mass. - Ratre spring and murommon early fall migrant. June 7-10; (.July 11) Aug. S-siept. 26.

R. I.- Ineommon migrant, manly in fall, coastwise. spring; Jul!: 30-Sejt. $1 \mathrm{~S}$.

Coxx- Lneommon migrant coastwise, mainly in fall. Ang. 24. 
LONGIPENNES.

$$
\text { RYNCHOPIDAE. }
$$

41. Rrichor's nigra Limmé.

\section{Black skimmer; Cut-water; Scissor-bill; Sea-crow; Sea-dog; Razor-bill.}

Linné, Syst. nat., ed. 10, 1758, vol. 1, p. 138. "AmericA. Audubon, Bircls of Amer., 18tt, vol. 7, p. 67, pl. 428.

Seacoasts; nests on sandy shores.

Me.-Accidental visitor: Matinicus Id.; off Saco, Aug. 31, 1879; Washington Co.; Wells Bay.

MAss.-Occasional summer risitor coastwise, said to have bred about 1830 at Muskeget Id.; easual inland (Springfield). July 16-Aug. 19.

Cons.-Accidental visitor: Stonington (Linsley).

TUBINARES.

PROCELLARIIDAE.

42. Fulaiarus glacialis (Limmé) Stephens.

Fulmar; Marbleheader; Noddy; Oil-bird.

Procellaria glacialis Limé, Fama Suec., ed. 2, 1761, p. 51. "IN MARI SEPTENTR. INTRA CIRCULUA ARCTICLM." Auduhon, Birds of Amer., 18tt, vol. 7, p. 204, pl. 45.5. Egg, Secbohm, 1896. p. 73, pl. 20 , fig. 5 .

Mainly pelagie; nests in shallow burrows or on cliffs.

ME.- Rare winter" visitor offshore: "coast of Maine," March, 1879.

MAss- Rare fall and winter visitor offishore: Georges Bank. Oct. 28-winter. 
AVES.

TCBINAREA.

$$
\text { PROCELLARIIDAE. }
$$

43. Puflixts borkalas Corv.

Cory's shearwater.

Cory, Bull. Nuttall ornith. chul), 1SS1, rol. (i, p. St. "NEAR Chathalishon, Cape C'on, Mrass."

Palagic; nest unknown.

MAss.- Uncommon summer and fiall visitor offishor. Ang. 2Nor.

R. I.- Uncommon summer and fall visitor offishore. Aug. 15Oct. 26 .

44. Perlines girats (O'Reilly) Salvin.

\section{Greater shearwater; Hag; Hagdon.}

Procellaria gravis O'Reilly, Ciroenland, aljacont seals, and northrest pass., 1\$1S, p. 140, pl. 12, fig. 1. "LATITUde of CAPE

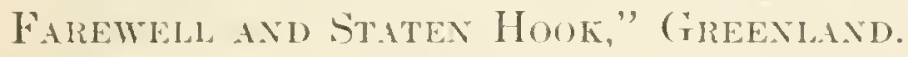

Puffims. cimerens Lath. Auduhon, Birds of Amer., 1St4, rol. 7, p. 212, pl. 456 .

Pelagix"; nest and rgogs nuknown.

ME.- Common stmmmer visitor offshore. Lug. 10.

X. II.-Summer visitor offshore: Portsmouth.

M.Ass-Common stmmer and fall visitor offishore; casual inland (1)orer). \ay 26-Xor. (? Dec.31).

R. I.- Lneommon summer and fall risitor offishore.

(i)x. - Encommon summer and fall visitor offishore.

45. PuFines grisletes (Gmelin) Finsch.

Sooty shearwater; Black hag or hagdon.

Godman, Monogr. petrels, 1908, pt. 2, p. 145, pl. 33.

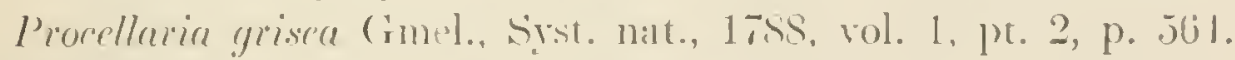

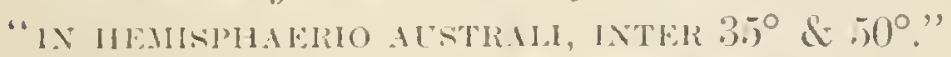

Pelagice; nests in burrows on sea islands. 
TUBINARES.

PROCELLARIIDAE.

Me.-Common summer risitor offshore. May-summer.

Mass.-Common summer and fall visitor oftshore. (Mar.) July 4-Oct.

R. I.- Lncommon summer visitor oftshore. (Mar. 2S) MaySept.

46. Aestrelata hasitata (Kuhl) Coles.

Black-capped petrel; Diablotin.

Procellaria hasitata Kuhl, Beitr. z. zool., 1S20, pt. 2, p. 142. No locality.

Oestrelata haesitata (Kuhl). Godman, Monogr. petrels, 190S, pt. 3, p. $18 t$, pl. 49.

Mainly pelagic; nests in burrows on islands.

I. H.-Areidental visitor": Pittsfield, about Aug. 30, 1893.

VT.-Accidental risitor: last of August, 1893 (place unknowu).

47. Daption capexsis (Limné) Stephens.

\section{Pintado petrel; Cape pigeon.}

Salvin, Cat. birds British mus., 1S96, rol. 25, p. 428. Gould, Birds of Australia, 1848, rol. 7, pl. 53.

Procellaria capensis Limné, Syst. nat., erl. 10, 175S, rol. 1, p. 132. "ad Can[item] is[onae] SPei."

Pelagic; nests in burrows on islauds of southern hemisphere.

ME.-Aceidental visitor: Iake Mooseluckmeguntic, Sept. 1872 (nec Harpswell).

4S. OCEANOdroma letconhoA (Vieillot) Stejneger.

Leach's petrel; Carey chicken; Mother Carey's chicken.

Procellaria lencorhioa Vieill., Nour. dict. d'hist. nat., 1517, rol. 25, p. 42.. "lles bords maritimes de la Prcardie," Fraxce. 
TUBINARES.

$$
\text { PROCELLARIIDAE. }
$$

Thalassidroma leachii 'T'emm. Audubon, Birds of Amer., 1844, rol. 7, p. 219, pl. 459. Egg, Capen, 18S6, pl, 24, fig. 1.

Pelagic; nests in colonies on islands, in burrows.

Mr. Abundant summer resident on outer islands from Casco Bay north. May-Oct. 21. Eggs, June 10-Aug. 19.

N. H.- Common migrant offishore; occasional inland in fall. Spring-Nor.

TT.-Accidental visitor: Ialke Champlain.

M.sss.-Common migrant offishore; casual inland in fall. June 21; Sept. 1-Nov. 25.

R. I.-Uncommon migrant offshore. June 9; Aug. 29-Oct. 14.

Coxs.- Uncommon migrant offshore, casual inland. Oct. 27.

49. OCeanites oceanicus (Kül) Lichtenstein.

\section{Wilson's petrel; Mother Carey's chicken.}

Procellaria ocpanica Kuhl, Beitr. z. zool., 1S20, pt. 2, p. 136, pl. 10, fig. 1. No locality given.

Thalassidroma wilsomii Bonap. Audubon, Birds of Amer., 1844, rol. 7, p. 223, pl. 460.

Pelagie; nests within Antaretic circle, in burrows.

Me.-Common summer visitor offshore; castual inland (near Augusta). Iune-Sept. 17.

N. II.-Common smmmer visitor offshore.

VT. - Accidental visitor: St. Albans (may hare beren O. lewcorhoa).

Mass.-Common smmmer visitor offislore. June 17-Sept. 23.

R. I.-Conmon stmmer visitor offshore. July-Aug.

Coxs.- Uncommon summer visitor offishore. 
STEGANOPODES.

SULIDAE.

50. Sula leucogastra (Boddaert) Sclater \& Salvin.

Booby; Booby gannet.

Pelecanus lewrogaster Bodd., 'l'abl. d. planch. enlum. d'hist. nat., 1783, p. 57. Based on Buffion.

Sula fusca Iinné. Audubon, Birds of Amer., 1844, vol. 7, p. $57, \mathrm{pl} .426$.

Seacoasts; nests on the ground.

Mass- Accidental risitor: Cape Cod, about Sept. 17, 187S (nee Essex Co.).

Coxx.- Linsley's record (Guilford) said to refer to S. bassana.

51. Stla Bassaxa (Limné) Boie.

\section{Gannet; Solan goose; White gannet.}

Audubon, Birds of Amer., 1St4, vol. 7, p. 44, pl. 425. Egg, Secbohm, 1896, p. 69, pl. 19, fig. 4.

Pelecanus bassanus Linné, Syst. nat., ed. 10, 175S, vol. 1, p. 133. "Ix Scotia, America."

Coastwise; nests in colonies on a few rocky islets of North Atlantic.

Me., N. H.-- Lncommon migrant. April-Nay; Sept.-Oct. 9.

Mass.-Common migrant oflshore. Mar. 26-June 7; Aug. 3Dec. 21.

R. I.- Uurommon migrant offshore. April-May 16; Sept. 10Oct. 26 .

Coxs.- Rare migrant offshore. 
STEGANOPODES.

PHALACROCORACIDAE.

52. P'halacrocorax carbo (Linné) Iseach.

Cormorant; Common cormorant; Shag; Taunton shag; Taunton turkey.

Audubon, Birds of Amer., 1S43, vol. 6, p. 412, pl. 415. Cones, Ké \o. Amer. birds, ed. 5, 1903, rol. 2, p. 963. Egg, Capen, 1SS6, pl. 21, fig. S.

Pelecanus carbo Linné, Sist. nat. ed. 10, 175s, vol. 1, p. 133. "is Europa."

Silt water mainly; nests in colonies on seal cliffs.

ME.-Common migrant and uncommon winter resident. Oct.April 20.

VT.- Rare migrant: Rutland, 1867.

MAss. - Uneommon migrant and rare winter resident coastwise. April-May 14; Sept. 22-Nor. (winter).

R. I.- Uncommon migrant and winter resident. Sept. 15(June 3).

Coxx-Cucommon migrant and winter resident. Fall-May.

53. Phalacrocoral atriten (I desson) Grant.

Double-crested cormorant; Shag; Taunton turkey.

Carbo auritu. Less., "l'mité d'ornith., 1S:31, vol. 1, p. 605. “NotVELLE-'KELLANDE."[!]

Phalacrororar dilophus Cones, Key No. Amer. birls, ed. 5, 19003, rol. 2, p. 963, fig. 676; Audubon, Omith. biogr., 1835, vol. 3, pl. 257. Jign, Capen, 18s6, pl. 22, fig. 1.

Coastal; nests in colonies on sea cliffs.

ME.-Common migrant; rare winter and summer (Black IIorse Lodgen resident. liggs, June 23-July 3.

N. H.- Cncommon migrant coastwise; occasional inland. Spring; Ort. 
STEGANOPODES.

PHALACROCORACIDAE.

Vt.- Rare migrant: Burlington, Nor.; Cambridge, Dec.; Windsor.

MAss-Common migrant, mainly coastwise. April 4-June 18 (July 7); Arg. 22-Nor. 24.

R. I.-Common migrant, mainly coastwise. April 22-May 22; (Aug. 21) Sept. 16-Nor.

Coxx-Lucommon migrant, mainly coastwise. April-May; mid-July-Nor. 13.

\section{PELECANIDAE.}

54. Pelecanes erythrorhychos Gmelin.

\section{American white pelican.}

Gmel., Sỵst. nat., 17Ss, vol. 1, pt. 2, p. 571. "1x Alierica SEITENTRIONALI."

Pelecanus americanus Audubon, Birds of Amer., 18tt, rol. 7 , p. 20, pl. 422 .

Salt and fresh water; nests on the groumd.

Me.-Accidental risitor: Calais; Eliot, pair, June S, 1897; Saponic Lalke, May 2S, 1892.

X. It.-Formerly a visitor (Belknap).

MAss.-Accidental risitor: Gloncester, 1S86; North Scituate, Oet. 5, 1876; Sandwieh, May 13, 1905.

55. Pelechues occinextalis (Linné) Richmond.

\section{Brown pelican.}

Pelecamus onocrotainss occidentalis. Limné, Sỵst. nat., ed. 12, 1766, vol. 1, p. 215. "Anerica."

Pelecanus fuscus Linné. Audubon, Birds of Amer., 1St4, vol. 7, p. 32, pls. 423,424 .

Coastal; nests on the gromnd or in low trees. 
STEGANOPODES.

PELECANIDAE.

Me.- The supposed Bar Harbor specimen was an escape brought from Sonth America.

Mass.-Accidental visitor: (? Ipswich, doubtful); Nantucket, about 186 i (flock of 13 ).

coxw-Areidental visitor: near New Haven.

FREGATIDAE.

56. Fregata aquila (Limné) Reichembach.

Man-o'-war bird; Frigate-bird; Man-o'-war hawk.

Pelecermus aquilus Limné, Sỵst. nat., cet. 10, 175s, vol. 1, p. 133.

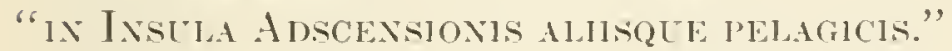

Tachypetes aquilus Linné. Audubon, Birds of Amer., 1St4, vol. 7, p. 10, pl. 421.

Coastal; nests in colonies, in low trees or on ground.

Me.- Doubtfully recorded from Boothbay.

Mass.-Accidental visitor: Now Berfford, Oct. 1ך, 1893 (record not confirmed).

Coxx-Acridental visitor: Fiulkner's I1., autumn, 1859.

ANSERES.

\section{ANATIDAE.}

57. Mergus americants Cassin.

American Merganser; Buff-breasted Merganser; Goosander; Pond shelldrake.

Cassin, Proce acadl. nat. sei. Phila., 1553, vol. 6, p). 1S7. Based on Wilson, no locality; probably New Jersey or Pennsylvania.

Mergus merganser Linné. Audubon, Birds of Amer., 1S43, vol. 6, p. 38т, pl. 411 . Eggr, Capen, 1886, pl. 21, fig. 5.

Mainly in fresh water; nests in hollow trees. 
ANSERES.

ANATIDAE.

ME.-Common migrant and winter resident; less common summer resident inland. Eggs, late May-Jume 19.

N. H.-Common migrant; less common summer resident from White MIs. north, and winter resident in open streams south of White MIts.

$V_{\mathrm{T}} \mathrm{-}$ - Uncommon summer resident; rare winter resident in open streams. Eggs, June 18.

Mass. - Uneommon migrant and winter resident, mainly on freshwater lakes and streams; said to have bred formerly. Oet.12May 1; summer (Springfield).

R. I.- Uneommon migrant and winter resident, mainly on ponds and rivers. Nor.-May 2.

Coxv- - Lncommon migrant and winter resident, mainly on ponds and rivers. Fall-April 17 .

5S. Mergus Serrator Linné.

\section{Red-breasted Merganser; Shelldrake.}

Linné, Srst. nat., ed. 10, 1758, rol. 1, p. 129. "IN Europa." Audubon, Birds of Amer., 1S43, vol. 6, p. 395, pl. 412. Egg Capen, 1S86, pl. 21, fig. 6 .

Salt and fresh water; nests on the ground.

Me.-Common migrant and winter resident coastrise; uncommon summer resident on coastal islands or inland likes. Eggs, June 21.

X. H.-Common migrant and winter resident manly coastwise. Nor:-April.

Vт.- Incommon migrant. April; Oct.

MAss.-Common migrant and winter resident, mainly coastwise; a few non-breeding birds summer offishore. Sept. 23-1 Lay 26 (summer). 
ANSERES.

\section{ANATIDAE.}

R. I.- Common migrant and winter resident roastwise; a few non-breeding birds smmmer offshore. Oct. 29-May 19 (smmmer).

Coss.-Common migrant and winter resident, mainly coastwise. Fall-April 14.

59. Iophontes ceclilates (Idimé) Reichenbach.

Hooded Merganser; Hairy-crown; Hairy-head; Hooded shelldrake; Sawbill diver; Water pheasant.

Mergus cucullatus Limmé, Srst. mat., ad. 10, 175S, rol. 1, p. 129. "Anerica." Audubon, Birds of Amer., 1St3, rol. 6, p. 402 , pl. 413. Egg, Capen, 1Ss6, pl. 21, fig. 7.

Fresh water mainly; nests in hollow trees.

ME.- Rare migrant and summer resident. Mar. 31-Mar’; summer; Sept.-late Nor.

․ H.- Rare migrant and summer resident (Lake L'mbagog). Mar.-Nor.

VT.-Rare summer resident. Mar. 27-Sept.

MAss.- Uncommon migrant. Mar. 1S-April 1S: (Aug. 11, Cambrilge) Oct. 7-Yor. 30 (D)e. 22).

R. I.- Encommon migrant and rare winter resident. Oct.Maly.

Coxx.- Tucommon migrant and rate winter resident. Nor:Mar. 6.

60. Anas phatringehos Limmé.

Mallard; Greenhead; Wild mallard.

Linné, Srst. nat., ed. 10, 1758, rol. 1, p. 125. "Ix Erropte MARITIMIS."

Anas bosclices Ijimne. Audubon, Birds of Amer., 1Sti, rol. 6, p. 236, pl. 385 .

Rivers, ponds, and in migrations, seacoasts; nests in marshes. 
ANSEREN.

$$
\text { ANATIDAE. }
$$

ME.-Rare migrant and winter resident. April; Oet. 1-Nov. 15 (winter).

N. H.- Rare migrant. Spring; Oct.-Nor.9.

VT.- Rare migrant.

Mass. - Uncommon migrant; rare in winter. Mar. 27-May 1; Sept. 22-Dee. 17 (Dec, 26, Jan. 9).

R. I.- Rare migrant and winter resident. Oct.-April.

Cosx.- Rave spring and uncommon fall migrant. Spring; Sept. 30-Nor.

61. Anas rubripes (Brewster) A. O. U. comm.

Black duck; Black mallard; Dusky duck; Red-legged black duck; Spring black duck; Winter black duck.

Anas olscura rubripes Brewst., Auk, 1902, vol. 19, p. 1St. "Lake Lamagog (NEw Hamphine SHORE)."

Anas obscura Cimel. Audubon, Birds of Amer., 1St3, rol. 6, p. 24t, pl. 386. Eggg, Capen, 1886, pl. 20, fig. 10.

Salt and fresh water; nests in marshes.

Me., N. H.-Common migrant, less common summer and (coastwise) winter resident. Eggs, April 28 (Me.).

Tr.-Common migrant, less common summer resident. Eggs, May.

Miss-Common migrant and winter resident (mainly coastwise); uncommon local summer resident. Eggs, April 19-May.

R. I.-Common migrant and winter resident, rare summer resident. Sept. 29-May; summer. Eggs, May 5-21.

Cons.-Common migrant and winter resident; rare summer resident. Sept.-May; summer.

62. Chactelasules streperes (Limné) Bonaparte.

Gadwall; Gray duck.

Ancts steepera linné, Syrst. nat., cd. 10, 1758, vol. 1, p. 125. "IN 
ANSERES.

ANATIDAL.

Ecropat: aques durcibus." Audubon, Birds of Amer., 1St3, rol. (6, p. 254, p). 3SS. Egg, Seebohm, 1896, p. 37, pl. 14, fig. 3.

Frosh and salt water; nests in marshes.

Me.- Rare migrant. April 20, IS79 (Scarborough); Oct. 2729.

N. H.- Rare migrant: Inmpton; Portsmonth, spring.

VT.- Ritre migrant: Otter ('reek, spring 1879); St. Albans.

Mass- Rare migrant. Oct. 2-ペッ. 1.

R. I. - Rare migrant. Feb. 26-. Iay 4; Oct. S-I)ece 1.

Coxx.- Rare migrant. Ang.-Oct. 14.

63. MIARECA PENELOPE (Linné) Sellỵ.

\section{Widgeon; European widgeon.}

Amas penelope Limé, Sist. 11at., erl. 10. 175S, vol. 1, p. 126. "Is

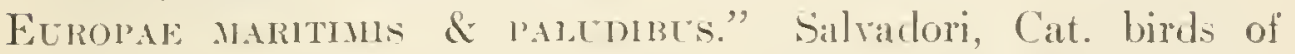
British mus., 1S95, vol. 27, p. 227. 1)resser, Birds of Europe, 1S71-81, vol. 6, pls. [432, 4333]. Figg, Seebohm, 1S96, p. 3S, pl. 13, fig. 4 .

Freshatral salt water"; nests on the groumd.

VT. - 1)oubtfully recorded.

Mass.-Aceidental visitor: Halilax, Oct. 20, 1999; Marblehead. Dec. 29, 1900; Matss. : (Silmmols).

R. I.- Ircidental visitor: Middletomn, Sept. 20, $18 S 9$.

6t. Marect americaxa (Gmeliu) Stepliens.

\section{Baldpate; American widgeon.}

Anas americana Gincl., Syst. 11at., 17Ss, vol. 1, pt. 2, p. j26.

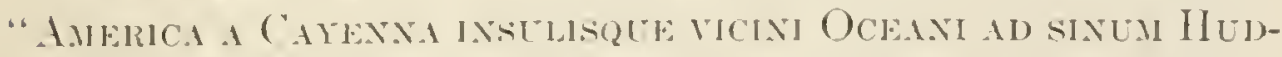
soxis." Amulubon, Birls of Amcr., 1843, vol. 6, p. 259, pl. 359.

Fresh and salt water'; nests in marshes. 
ANSERES.

ANATIDAE.

Me.-Uncommon migrant, mainly constwise; aceirlental in winter. Mar. 1S-April; Sept.-Nor. 1S (Feb. 20, 1SSt, Freeport).

N. H.- Uncommon migrant. April; Sept.-Oct.

VT.- Rare migrant.

Mass- - Uncommon migrant and rare winter resident (Nantucket). Mar. 22-April 17; Sept. 6-Dec. 2 (winter).

R. I.- Uncommon migrant. ? April; Sept. 20-early Dec.

Coxs.- Uncommon migrant.

65. Nettion crecca (Linné) Kaup.

\section{European teal; Teal.}

Salvadori, Cat. birds British mus., 189.5, rol. 27, p. 243.

Anas erecca Linné, Syst. mat., ed. 10, 175S, vol. 1, p. 126. "IN EUROPAE AQUIS DULCIBUS."

Querquedula crecea (Limné). Dresser, Birds of Europe, 187181, vol. 6, pl. [426]. Egg, Seebohm, 1596, p. 40, pl. 16, fig. 3.

Fresh water mainly; nests in marshes.

Me.-Aceiflental visitor: Caseo Bay, April 6, 1903.

Mass.-Accidental risitor: "Massachusetts" (Samuels); Muskeget Id., Mar. 16, 1890; Sagamore, Feb. 20, 1896.

Cons- - Aecidental risitor: East Hartford, Nor. 14, 1859.

66. Nettion carolinensis (Gmelin) Baird.

\section{Green-winged teal.}

Anas carolinensis Gmel., Sỵst. nilt., 1785, rol. 1, pt. 2, p. 533. "in Americale, a Carolina ad singa Hudsonis, stagnis siluaTicis." Audubon, Birds of Amer., 1843, vol. 6, p. 281, pl. 392. Fgg, Capen, 1SS6, pl. 20, fig. 11.

Fresh and salt water; nests in marshes.

Me.-Commou nigrant. April; Sept. 15-Nor. 15.

N. H.- Ǐncommon migrant. 
ANSERES.

ANATIDAE.

Vr.-Common migrant; (?"smmmer resident"). Mar.; Sept. MAss.- I neommon spring and rare fall migrant; accidental in winter. April 4-23; Sept. (j-I)er. 13 (Jan. 1; Feb. 1, 1892, West Barnstable).

R. I.- Lncommon migrant; rare in winter. Mar.; Sept. 2Nov. 7 (1)ec. 30).

Coxx.- Incommon migrant and rare winter resident. FallMar.

67. Querquedtat hiscous (Timé) Stephens.

\section{Blue-winged teal.}

Anas discors Linné, Syst. nat., ed. 12, 1766 , rol. 1, p. 205. "IN America septentrionalı," Aurubon, Birds of Amer., 1843, vol. 6, p. 287, pl. 393. Eggg, Capen, 1886, pl. 20, fig. 12.

Fresh and salt water; nests in marshes.

ME.-Common migrant and in Washington Co., a ralre summer resident. (Mar. S) April-May ; summer; Sept. 15-Oet. 16.

N. II.-Uneommon migrant. May; Ang. 15- \or.

VT.- Lneommon migrint; (" "stummer resident"). AprilMisy; fall.

Mass. - Rare spring and common fall migrant; probably once bred. April 11-.Iay 31; (June S and 20, Cambridge); Aug. 16Nov. 26.

R. I.- Rare spring and uncommon fall migrant and raresummer resident (at Silkonnet). MIay (summer); Xug. 27-Dor. 5. Eggge, May.

Coxs.- Rare spring and uncommon fall migrant. Spring; Alig.-Oct. 20 .

6.9. Spatela chepata (Iimé) Boik.

\section{Shoveler; Spoonbill.}

Anas clypeata Linné, Syst. nat., ed. 10, 175S, vol. 1, M. 124. "IN 
ANSERES.

\section{ANATIDAE.}

Europae maritinis." Audubon, Birds of Amer., 1St3, vol. 6, p. 293, pl. 394. Iigg, Secbohm, 1S96, p. 42, pl. 13, fig. 2.

Fresh and salt water; nests in marshes.

Me.- Rare migrant coastwise. April 14-23; Sept.

\. H.- Rare migrant: Hampton; Rye Beach, Ang. 1872.

$\mathrm{T}_{\mathrm{T}}$ - Rare migrant.

Mass. - Rare migrant. Spring; Sept. 17-Nor. 7.

R. I.- Rare migrant. April 10; Oct. 2-2S.

Conx.- Rare migrant. (Late July) Ang.-Dec. S.

69. Dafila acuta (Limé) Bonaparte.

Pintail; Gray duck ( + ); Pheasant duck; Sprigtail.

Anas acuta Limné, Syst. nat., ed. 10, 1758, vol. 1, p. 126. "1N Europae marituis." Audubon, Birds of Amer., 1S43, p. 266. pl. 390. Egg, Secbohm, 1896, p. 35, pl. 13, fig. 3.

Fresh and salt water; nests in malshes.

Me.- Rare migrant. Late Mar.-April; Sept. 20-Nor. 7 (late Yov.).

N. H., VT.- Rare migrant.

MAss. - Ralre migrant. (Feb. 21) Mar. S-April 3; Sept. 11Dec. 21.

R. I.- Uncommon migrant coastwise. Spring; Sept. 4.

Coxx.- Uncommon migrant; (? rare winter resident). Mar. 23 ; fall.

70. Arx spoxsa (Linné) Bonaparte.

Wood duck; Bride duck; Rainbow duck; Regal duck; Summer duck.

Allas sponsa Linné, Syst. nat., ed. 10, 1758, vol. 1, p. 12S. "IN America septextrionali." Aulubon, Birds of Amer., 1843, rol. 6, p. 271, pl. 391. Eggg, Capen, 1S86, pl. 21, fig. 1. 
ANSERES.

\section{ANATIDAE.}

Freshwater streams and ponds; nests in hollow trees, larely on the groumd.

Me.- Lncommon migrant and summer resident. Mar. 2Slate Nov.

․ H.- Eucommon migrant and summer resident. Mar.-Dec.

TT.- Lnconmon migrant and summer resident. April SOct. 23.

M.ıss.- Uncommon migrant and rare summer resident; accidental in winter. (Mar. 10) Mar. 20-April 30; smmmer; Sept. 15-Nov. 18 (Dec. 31, 1S90, So. Weymouth; .Jan. 11, 1SSS. 'Tamton). Eggs. April 23-May ?.

R. I.- Uncommon migrant and rale summer resident. Mar.Nor. 11. Eggs, May 10.

Coxi- Lucommon migrant and rare summer resident; accidental in winter. Mar. 18-Oet. (.tan. 9, 1880, Norwich). Eggs, Mar.

71. Marlla americana (Eytom) A. O. U. comm.

\section{Redhead; American pochard.}

Fuligula americana livt., Monogr. Anatidae, 1S3S, p. 155. "North Amlirica."

Fuligula ferina Limné. Andubon, Birds of Amer., 1843, rol. 6, p. 311 , pl. 396 .

Fresh and salt water; nests in marshes.

ME. - Rare migrant; said formerly to have bred in Washington Co. Mar. 27; Sept. 21-Nov. 5.

\. H.- Rare fill migrant.

VT.- Rare fall migrant: Lake. Champlain.

MAss.- Rale migrant more frequent in fall, and rare winter resident on south coast. Mar. (i-April 3; Oct. 1-1)ec. 21 (winter).

R. I. - Rare migrant and winter resident. Ort. S-Fib. 3.

Coxis.- Rare migrant. 
ANSERES

\author{
ANATIDAE.
}

72. Marila vallisseria (Wilson) A. O. U. comm.

\title{
Canvas-back.
}

Anas valisineria Wils., Amer. ornith., 181t, vol. 8, p. 103, pl. 70, fig. 5. "Hudson and Delawaire... rivers.... and in the neighboctiood of the Chesapeake Bay."

Fuligula valisneriana (Wils.). Audubon, Birds of Amer., 1843 , vol. 6 , p. 299 , pl. 395.

Fresh and salt water; nests in marshes.

ME.- Very rare fall migrant.

VT.- Very rare migrant: Lake Champlain (Perkins).

Mass - Rare migrant. Mar. 11-31; Oct. 19-Dec. 18 (Jan. 8, Cambridge).

R. I.-Rare migrant. April; Oct. 2-Nor. 16.

Cons.- Rare migrant. May 7.

73. Marila marila (Linné) A. O. L. comm.

Greater scaup; Blackhead; Bluebill; Bluehead; Broadbill; Raft duck; Widgeon.

Anas marila Limné, Syst. nat., ed. 12, 1766, vol. 1, p. 196. "IN Europae alpinis in Svecia, Norvegia, Austria."

Fuligula marila (Limé). Audubon, Birds of Amer., 1S44, rol. 7 , p. 355, pl. 498. Egg, Seebohm, 1896, p. 46, pl. 14, fig. 2.

Fresh and salt water; nests in marshes. .

Me.-- Common coastwise migrant; rare in winter. Oct.Nov.; (winter); Mar.-April.

N. H.- Uncommon migrant, mainly coastwise. April; Oct.Dec. 13.

VT.- Lineommon migrant at Lake Champlain.

MAss. - Rare spring, and common fall migrant; winters locally on coast. Mar. 27-May 1; Sept. 18-Dec. 26; winter. 
ANSERES

$$
\text { ANATIDAE. }
$$

R. I.-Common migrant and less common winter resident coastwise. Oct. 1-May 1 (July 19, 1900, cripple).

Coxs.-Common migrant and uncommon winter resilent coastwise. Oret.-May 17 (June).

74. Marila arisis (Eytom) Bonaparte.

Lesser scaup; Blue-billed shoveler; Creek broadbill; Little blackhead; Little bluebill; Little raft duck; River broadbill.

Fuligula affinis Eyt., Monogr. Anaticlac, 1835, p. 157. "Nortн AMERICA."

Fuligula marila (Limné). Audubon, Birds of Aner., 18t3, rol. 6. p. 316, p). 397.

Aythya affinis (Evt.). Chapman, Handb. birds east. No. Aner., 1895, p. 112.

Fresh and salt water; nests in marshes.

Me.-Rare migrant. Mar.-April; Oct.-Nor.

N. H. - Rare migrant.

VT.- Rare migrant: Iake Champlain; Otter Creek, May 19, $1 S S 3$.

Mass. - Rare spring and common fall migrant; rare winter resident on south coast. Mar. 2-April 14 (May 5); Oct. S-Dee. 6 (winter).

R. I.- Uncommon migrant and winter resiclent. Oct. 15-April.

Cosx.- Lncoummon migrant and winter resident. Oct.-Maly 17 (June).

75. Marila COLlaris (Donovan) Bonaparte.

\section{Ring-necked duck; Ring-neck scaup.}

Anas rollaris 1)onov., British birds, 1809 , vol. 6, pl. 147 and text.

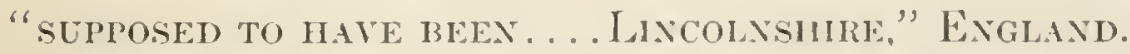

Fviligula rufitorques Bonap. Audubon, Birds of Amer., $18 t 3$, rol. 6 , p. 320 , pl. 395 . 
ANSERES.

ANATIDAE.

Aythya collaris (Donor.). Chapman, Handb. birds east. No. Amer., 1895, p. 112.

Fresh and salt water; nests in marshes.

ME.- Rare migrant; once bred in Washington Co. Mar. 31May 1; (summer); Oct. 11-Nor. 2.

N. H.- Rare migrant: Concord, Nor.; Little Bay near Portsmouth.

Mass.-Rare migrant. Mar.-April; Ort. 4-Nor. 30.

R. I.- Rare migrant. Nor. 11.

Cons.- Rare migrant.

76. Clangula ciangula americana (Bomaparte) Faxon.

\section{American goldeneye; Garrot; Whistler; Whistle-wing.}

Clangula americana Bonap., Geogr. and comparative list birels Europe and No. Amer., 1838, p. 5S. "America."

Fuligula clangula (Iinné). Audubon, Birels of Amer., 1St3, vol. 6, p. 362, pl. 406. Egg, Capen, 1SS6, pl. 21, fig. 2.

Fresh and salt water; nests in hollow trees.

Me.-Common migrant and winter resident coastwise; less common summer resident in northern part. Egos, May 27-June S.

N. H.- Common migrant and winter resident coastwise or in open rivers; uncommon summer resident (Lake Lmbagog).

VT.-Common migrant on Lake Champlain and Connecticut River.

MAss.-Common migrant and winter resident mainly coastwise. Oct. S-May 1 (July 26, Boston).

R. I.-Common migrant and winter resident coastwise. Nor. 15-April 20.

Covn.- Common migrant and winter resident manly coastwise. Late Oct.-April 16. 
ANSERES.

\section{ANATIDAE.}

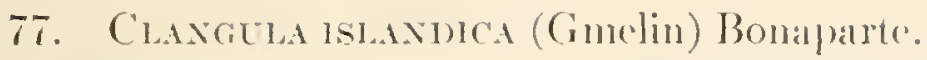

\section{Barrow's goldeneye.}

Anas islandica Gmelin, Sỵst. 11at., 17SS, rol. 1, pt. 2, p. 541. "IN Islatidia."

Clangula barrovii Swanson and Richardson, Fanna BorealiAmer., 1S31, vol. 2, p. 456, pl. 70.

Fresh and salt water; nests in hollow trees.

ME.- Rare winter visitor coastwise. Jan--April.

N. H. - Rarre winter visitor: Little Bay, Portsmonth.

Vt. Erroneously recoreled.

MAss. - Rare winter visitor coastwise. Dec. 7-Feb. 22.

Coxs.- Rare winter visitor coastwise: off Portand, Nov. 1t, $1 S 67$.

7S. CinkitonetTa Alibolat (I imné) Stejnegrer.

Bufflehead; Bumble-bee duck; Butterball; Devil diver; Dipper; Hell diver; Spirit duck.

Anas albrola Linné, Syst. nat., al. 10, 1758, vol. 1, p. 124. "AnwkICA."

Fuligula albrola (Limmé). Anchubon, Birds of Ancr., 1S43, rol. 6. p. 369, pl. tos.

Fresh and salt water; nests in hollow trees.

Mr.- Common migrant and on sonth coast an uncommon winter resident; said to nest in Washington Co. I ate Oct.-April (May 11) (stmmere).

N. H.- Uncommon migrant and rare winter resident chicefly coastwise. Mall.-April; O(t.-- Nov. (winter).

TT.- Encommon migrant.

Mass. - Incommon miglant and lanre wintere lesident. Mar. 11April S; Oct. 9-1)ec. 23 (winter). 
ANSERES.

ANATIDAE.

R. I.- Uncommon migrant and rare winter resident coastwise. April; Oct. 13-Dec. 7 (winter).

Coxv. - Uncommon migrant and winter resident. Nor.-April 15 .

79. Harelda hyemalis (Limné) L. Brehm.

Old squaw; Long-tailed duck; Old injun; Old wife; Scoldenore; Scolder; South-southerly.

Anas hyemalis Limné, Syst. nat., ed. 10, 1758, vol. 1, p. 126. "IN Europa \& America arctica."

Fuligula glacialis (Linné). Audubon, Birds of Amer., 1843, vol. 6, p. 379, pl. 410. Egg, Seebohm, 1896, p. 50, pl. 15, fig. 3.

Salt and fresh water; nests on the ground.

ME.-Common migrant and winter resident coastwise; a few non-breeding birds summer. Late Nor.--May 21 (summer).

N. H.-Common migrant and winter resident, chiefly coastwise.

VT.- Rare migrant to Lake Champlain and Connecticut Valley. Oct. 24 .

Mass - Common migrant and winter resident, mainly coastwise. Oct. 10-May 22 (June 17).

R. I. - Common migrant and winter resident. Nov. 7-May 4. Cons.-Common migrant and winter resident coastwise. Oct.-May.

80. Histrionicus histrionices (Linné) Boucard.

\section{Harlequin duck; Lord-and-lady; Sea mouse.}

Anas histrionica Linné, Sysst. nat., ed. 10, 1758, vol. 1, p. 127. "Anierica."

Fuligula histrionica (Limné). Audubon, Birds of Amer., 1S43, rol. 6, p. 374, pl. 409. Egg, Seebohm, 1896, p. 49, pl. 15, fig. 1.

Fresh and salt water; nests on the ground or in hollow trees. 
ANSERES.

ANATIDAE.

ME.- Uneommon winter resident about rocky islands. Oct. 19-NIar. (April).

N. H.- Rare winter visitor coastwise. Nor.

MAss. - Rare winter risitor coastwise; calsulal inland. Nor. 1Jin.

R. 1.-Rare winter visitor coastwise. Nor. 2S-Jan. 29.

S1. Camptorhychus mabadorits (Gmelin) Bonaparte.

Labrador duck; Pied duck; Sand-shoal duck.

Anas labradoria Gmel., Sist. nat., 17Ss, vol. 1, pt. 2, p. 537. "in America boreali."

Fuligula labradora Lath. Audubon, Birds of Amer., 1S43, vol. 6, p. 329, pl. 400.

Formerly on seacoast; nests on "tops of low fir trees." Last specimen taken in 1875 .

Me.- Formerly a winter visitor coastwise.

Mass.-Formerly a winter risitor coastwise; last taken in the State at Swampscott, early September, 1862.

Coxw.- Formerly a winter resident (Stratford).

S2. Somateria iollissina Borealis (C. L. Brehm) A. E. Brehm.

\section{Northern eider; Greenland eider.}

Platypus borealis C. L. Brehm, Lehrb. naturga. europ. vögel,

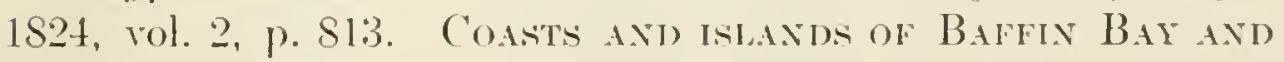
Davis Strkit (fide H. C. Oberholser).

Somateria mollissima (linné). Baird, Brewer, and Ridgway, Water birds No. Amer., 1SS4, vol. 2. p. 72, figs. on p. 77.

Salt water chiefly; nests on the ground.

Mr.- Rare winter visitor constwise. Oet. 19-April 6.

Mass. - Rare winter visitor coastwise. Late Oct.-April 3.

R. I.- Unsatisfactorily recorded from Newport. 
ANSERES.

$$
\text { ANATIDAE. }
$$

S3. Somateria Dresseri Sharpe.

\section{American eider; Canvasback; Sea duck; Wamp.}

Sharpe, Ann. mag. nat. hist., 1871, ser. 4, rol. 8, p. 51. [NorTH A.IERICA].

Fuligula mollissima (Limmé). Audubon, Birds of Amer., 1St3, rol. 6, p. 349, pl. 405. Egg, Capen, 18s6, pl. 21, fig. 3.

Salt water chiefly; nests on the gromnd.

Me.-Common winter, ant from Isle au Haut north, rare summer resident. Nor-April; summer. Eggs, June 15-July 4.

‥ H.- Uncommon migrant and winter resident coastwise.

MAss.-Uneommon migrant and winter resident coastwise. Nor. 5-April 19.

R. I.- Uncommon migrant and winter resident coastwise.

Coxx.- Rare winter resident coastrise. Fall-(May 29).

S4. Somateria spectabilis (Iimné) Leach.

\section{King eider.}

Anas spectabilis Limné, Srst. nat., et. 10, 1758, rol. 1, p. 123. "IN CANADA, Svecha."

Fuligula spcctabilis (Linné). Andubon, Birds of Amer., 1843, rol. 6, p. 347, pl. 404. Egg, Seebohm, 1896, p. 54, pl. 9. fig. 1.

Salt water chiefly; nests on the ground.

ME.- Uncommon winter resident coastwise. Dec. 11-April 15 (Mal 29$)$.

X. H.- Rale wintel visitor: Hampton.

MAss.- Rare winter visitor coastwise. Oct. 21-April 5.

R. I. - Rare minter visitor constwise. (Sept. 15) Nor. 27-Feb.

Coxy.- Rare winter risitor coastwise. Nov. 4. 
A.SELRES:

\section{A.ATIDAE.}

S5. Ondmina Ambrichat Swainson.

\section{American scoter; Black coot; Butterbill; Butterbill coot; Yel- low-billed coot.}

Swains., in Swains. and Rich., Famma Boreali-Amer., 1831, rol. 2, p. 450. "HUDSON's B.tY, LAT. $57^{\circ}$."

Fuligula americana (Swains.). Audubon, Birds of Amer., 1843. rol. 6, p. 343, pl. 40:3.

Salt and fresh water; nests on the ground.

ME., N. H.-Common migrant and winter resident mainly coastwise; a few non-brealing birls smmmer oftshore.

VT.- Lncommon migrant: St. Johnsbury; Windsor.

MAss.-Common migrant and winter resident; a few nonbreeding birds summer off'shore. Sept. S-1Lay 24 (summer).

R. I.- Common migrant and winter resident coastwise. Ort.May 11.

Coss.-Uneommon migrant and winter resident. Oot. t-Maỵ.

S6. OIdenita deglaxd Bonaparte.

\section{White-winged scoter; May white-wing; Velvet duck; White- winged coot.}

Bonaly., Rev. crit. dr l'orn. Burop. de Dr. Degland, 1S50, p. 105. "A.HERICA."

Fuligula fusea (Limné). Audubon, Birds of Aner., 1St3, vol. (i. p. 332 , pl. 401.

Salt and fresh water"; nests on the gromel.

ME., \. h.- ('ommon migrant and winter resident mainly coastrise; a few non-breeling birels summer offshore.

YT.- Rare migrant: Mirlollebury.

Mass.- Common mignant and winter resident manly coatstwise; a few non-breeding birls smmmer oftshore. Sept. 6-May 2.) (sumner). 
ANSERES.

ANATIDAE.

R. I.-Common migrant and winter resident, mainly coastwise; a few non-breeding birls summer offshore. Sept. 15-May; summer.

Coxs. - Abundant migrant and common winter resident, mainly coastwise; a few non-breeding birels summer offshore. Oct. $4-$ May 15; summer.

S7. Oidenia perspicillata (Limné) Stephens.

Surf scoter; Baldheaded coot;- Box coot; Gray coot; Hollowbilled coot; Patch-bill coot; Patch-poll coot; Sea coot; Skunk-head.

Chapman, Handb. birds east. No. Amer., 1895, p. 118.

Anas perspicillata Limné, Syst. nat., ed. 10, 1758, vol. 1, p. 125. "CANADA."

Fuligula perspicillata (Limné). Audubon, Birds of Amer., 1843, rol. 6, p. 337, pl. 402. Egg, Seebohm, 1896, p. 52, pl. 15, fig. 2.

Salt water mainly; nests on the ground.

Me., X. H.-Common migrant and winter resident mainly coastwise; a few non-breeding birk summer offshore.

Vr. - Rare migrant: Bradford.

Mass.-Common migrant and winter resident coastwise; a few non-breeding birds summer offshore. Sept. t-May 9; summer.

R. I.-Common migrant and winter resident coastwise; a few non-breeding birds summer offshore. Sept. 1-Nay 21; summer.

Coxv-C Common migrant and winter resident; a few non-breeding birds summer offishore. Sept.-April; summer.

SS. Emismatura Janatersis (Gmelin) Salvadori.

Ruddy duck; Booby; Broadbill; Dumb-bird; Foolish coot.

Anas jamaicensis Gmel., Surst, nat., 17S\&, vol. 1, pt. 2, p. 519. "JAIIAICA." 
ANSERES.

\section{ANATIDAE.}

Fuligula mbida Wils. Audubon, Birds of Amer., 1St3, rol. 6, p. 324, pl. 399. Egg, Capen, 1Ss6, pl. 21, fig. 4.

Fresh water chiefly; nests in marshes.

Me.- Lncommon migrant, and rare smmmer resident. AprilMay,; (summer); Oct.-Nor.

N. H.- Uncommon migrant, rare in summer; may brecel (Newfound Lake, July 11, 1901; Rye Beach, Aug. 22, 1879).

VT.- Uncommon migrant and rare summer resident (Lake Memphremagog).

Mass.-Common migrant; rery rare summer resident (Calpe Cod). Mar. 20-(summer); Sept. 30-1)ec. 1t (1)ec. 25, 1902, Woods Hole; Jan. 9, 1909, Jamaica Plain).

R. I.-Common migrant and occasional summer resident. Mar.; Oet. 1-Dec. 22.

Coxs - Common migrant, mainly in fall; rare in summer (June 5, 1S93, flock of 5, Portand). Spring; Oct.-I)ec. 25.

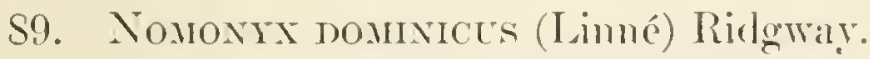

Masked duck.

Ridgway, MIanual No. Amer. lirels, 1SS7, p. 114.

Anas domimira Limné, Sist. nat., ed. 12, 1766, p. 201. "1x AMERICA MERIDIONAII."

Erismatura dominira Eyt. Baird, Cassin, and Iawrence, Birds of No. Amer., 1870, vol. 2, pl. 92.

Fresh water; nests among rushes.

VT.- Accidental visitor (or escape): Alburgh Springs on Iakhe Champlain.

MAss- - Acridental visitor: Malden, Aug. 27, 1S\$!).

90. Cumin mimbrorea (Pallas) Boie.

Lesser snow goose; Mexican goose; Wavy; White brant.

Ridgray, Manual No. Amer. birds, 18S7, p. 115. I)resser, 
ANSERES.

$$
\text { ANATIDAE. }
$$

Birds of Europe, 1S71-S1, vol. 6, pl. [417], lower fig. Egg, Seebohm, 1S96, p. 30, pl. 11, fig. 3 .

Anser liyperboreus Pall., Spicilegia zool., 1769, fasc. 6, p. 25. "IN TERRIS BOREALIBLS, AD ORIENTEM 130". LONGITLdinis, SIVE CIRCA INAMA ET IANAM FLUVIOS."

Salt and fresh water; nests on the ground.

ME.- Rare fill migrant. Oct. 2-Dec.

‥ H.- Rare fall migrant: Lake Umbagog, Oct. 2, 1896; Seabrook.

VT.- Rare fall migrant: Iake Champlain; ? Lunenburg; St. Jolinsbury.

MAss.- Rare migrant. April 13, 1908, 'Townsend; (? July) Oct. 15- Nor. 21.

R. I.- Rire fall migrant. Sept. 3-Oct. 16.

Cons.- Rare fall migrant: Silybrook, fall, IS75; Stratford, Oct. 5, $18+2$.

\section{Chen hyperborea xiralus (Forster) Ridgway.}

\section{Greater snow goose; Wavy.}

Ridgway, Manual No. Amer. birkls, 18S7, p. 115.

Anas nivalis Forster, Phil. trans. roy. soc. London, 17\%:, vol. 62, p. 413. "Setery River," Hudsox Bay.

Anser hyperborews Ginel. Autubon, Birds of Amer., 1St3, rol. 6, p. 212, pl. 381, fig. 1.

Salt and fresh water; nests on the grount.

Me.- Now an accidental visitor: Georgetown, April 25, 1903; mouth of Kennebec River, April 7, 1890; Lubec, April 30, 1906.

Mass.- - Now an accidental risitor: near Springfield (Morris); rarious doubtful records.

Conn. - Now an accidental visitor: Stratford, three wintered, 1S67-6S (fide Averill); also listed by Linsley (1S43), but may have been $C$. hyperborea. 
ANSERES.

$$
\text { ANATIIAE. }
$$

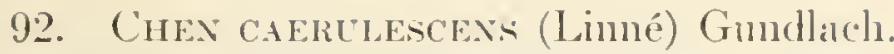

\section{Blue goose; Blue wavy.}

Ridgray, Maumal No. Amer. birds, 1SS7, p. 114. Elliot, New and heretofore unfigured birls No. Aner., 1S69, vol. 2, pl. 43.

Anas caerulescens Limé, Syst. nat., ed. 10, 1758 , vol. 1. p. 124. "Caxidot."

Salt and fresh water; nests on the gromul.

․ H.- Rare fall migrant: Iake Lmbagog, Oct. 2, $1896^{\circ}$

Mass- Rare fall migrant: Test Gloncester, Oct. 20, 1876.

R. I.- Ralre migrant: Westerly, Marr. 16, 1S94. Other records in error for C. hyperborea.

93. Axser almifrons gamber (Hantlaub) Coues.

\section{American white-fronted goose; Laughing goose.}

Anser yambelli (sic) Hartl., Rev. and mag. zool., ser. 2, 15.52, vol. t, p. 7. "TExts."

Anser albifrons Bechst. Andubon, Birds of Amer., 1S 13, vol. 6, p. 209 , pl. 38() .

Fresh and silt water"; nests on the ground.

VtT.- Doubtfully recorded.

Mass.-Aecillental migrant (? formerly regular): Ipswich, Aug. 1907; Plymouth, Nor. 26, 1897; Quiney.

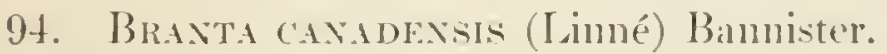

\section{Canada goose; Wild goose.}

Anas canarlensis I inné, Syst. nat., erl. 10, 175s, rol. 1, p. 123. "CA.NAD.t."

Anser canalensis (Limné). Andubon, Birls of Amer., 1St3, rol. 6, p. 17s, pl. 376. Egg, Scebohn, 1596, p. 35, pl. 12. fig. 2.

Fresh and salt water; nests on the gromnd, landy in trees. 
ANSERES.

\section{ANATIDAE.}

Me.-Common migrant. (Feb. 28) Mar. 5-May; Oct. 13Dec. 8 .

N. H., VT.-Common migrant. Mar. 11-April; Oct.-Dec. 7.

MASs.-Common migrant, formerly summer resident (said to have bred at Lexington, late April, 1Sss); may winter rarely on south coast. Mar. S-June 5; (Aug. 3) Sept. 21-Dee. 30 (Jan. 10; Jan. 31).

R. I.-Common migrant. Mar. 13-May 7; Sept. 17-Dec. 29.

Conn.-Common migrant and less common winter resident. Mar.-May 22; Oet.-winter.

\section{Branta Canadevsis hutchissil (Richardson) Coles.}

\section{Hutchins's goose; ? Southern goose.}

Coues, Key to No. Amer. birds, ed. 5, 1903, vol. 2, p. 905.

Anser hutchinsii Rich., in Swainson and Rich., Fauma BorealiAmer., 1831, vol. 2, p. 470. "Melville Peninsula," Arctic Anerica. Audubon, Ornith. biogr., 1835, vol. 3, p. 226, pl. 277.

Fresh and salt water; nests on the ground.

Me.-Accidental migrant: Cape Elizibeth, Nor. 13, 1894; Portland, spring 1599; Rangeley.

Mass.- Supposed to oecur as a migrant.

Conn.- ? Uncommon fall migrant; said to arrive later than B. eanadensis and to pass at once south (Merriam, 1877).

96. Branta bericha glalcogastra (C. L. Brehm) Coles.

\section{White-bellied brant; Blue brant; Brant; May brant.}

Berniela glancogaster C. L. Brehm, I Iandb. d. naturgesch. aller vögel Deutschl., 1\$31, p. S49. "Aus Deм hohen Norden m Herbste an me Küsten der deutschen Ostsee."

Anser berniela Linné. Audubon, Birds of Amer., 1843, vol. 6, p. 203, pl. 379 .

Salt water; nests on the ground. 
ANSERES.

ANATIDAE.

Mr.-Common coastwise migrant. April 27-May; Oct.-Nov.

․ H.- Uncommon coastwise migrant, casmal inlanel.

VT.- Rane migrant.

MAss. - Common coastwise migrant; uncommon winter resident on sonth coast. Mar. 12-May 17 (June 2); Sept. 12-Dec. 12 (winter).

R. I.-Common migrant and rare winter resiclent offishore. Mar. 2t-April 2S; Nor.-Dec. 16 (winter).

Coxs.-Common migrant and occasional winter resident offshore. April 14-May; Oct. 15-(winter).

97. Branta nigricass (Lawence) Bannister.

\section{Black brant.}

Anser nigricans Lawr., Ann. lyeeum nat. hist. New York, $18+6$, vol. 4, p. 171, pl. 12. "EgG Harbor, Х. J."

Seacoasts; nests on the ground.

MAss.-Accidental risitor: Chatham, spring, 1883.

9S. Branta hecopols (Bechstein) Bannister.

\section{Barnacle goose.}

Anas lencopsis Bechst., Ornith. taschb. 1)eutschl., 1803, p. 424.

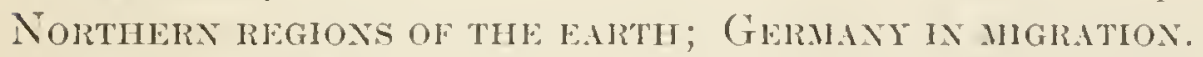

Anser lencopsis (Bechst.). Audubon, Birds of Amer., 1S43, vol. 6, p. 200, pl. 37S. Egg, Scchohm, 1896, p. 34, pl. 10, fig. 2.

Seacoasts and grassy flats; nests on the ground among roeks.

Me.- Doubtfully reported from near Portland about 1STS.

VT.-Accidental visitor: Marshfield, 1878.

MAss.-Aecidental risitor: North Chatham, Nor. 1, 1895.

Cons:- Recorded from Stonington (Linsley). 
ANSERES.

$$
\text { ANATIDAE. }
$$

99. Olon cranes (Linné) Bonaparte.

\section{Whooping swan; Whooper; Wild șan.}

Anas ryguus Linné, Syst. nat., ed. 10, 1758, vol. 1, p. 122. "IN Europa, Aimerica sempentrionali."

Cygmus musicus Bechst. Dresser, Birds of Europe, 1871-S1, vol. 6, p. [433], pl. [419], fig. 4. Egg, Seebohm, 1S96, p. 29, pl. 7 , fig. 1 .

Seacoasts, lakes, swamps; nests on the groumd.

Me.-Accidental visitor: Poke-a-moon-shine Lake, Waslington Co., Sept. 10, 1903.

\section{Olor columbianus (Ord) Stejneger.}

\section{Whistling swan; American swan.}

Anas columbiamus Ord, in Guthrie's Geogr., hist., and commercial grammar, 2d Amer. ed., 1815, p. 319. Based on Lewis and Clarke: "Below THE GREAT NARROW's of the COLUMBdA."

Cygmus americanus Shappless. Audubon, Birds of Amer., 1S43, vol. 6, p. 226, pl. 3S4.

Salt and fresh water; nests on the gromul.

Me.- Unsatisfactorily recorded from mouth of Kennebec River Nor., 18s1; and near Crawford Lake, Sept. 10, 190:3.

N. H.- Rare migrant: Great Bay (bis), Dec. 16, 1902, and-; Seabrook, Oct. 18, 1S7S.

VT.- Rare migrant: Alburg, Lake Champlain.

MAss. - Rare migrant and winter resident (at Nantucket) formerly commoner. Oct. 16-Dec. 27 (winter-Mar. 4).

R. 1.- Rare migrant: Quonocontang Pond, Nov. 9, 1879; IVesterly, Nov. 13, 1879 .

Cons.- Rare migrant. Middle Mar.; fall-Nov. 2. 
ANSERES.

ANATIDAF.

101. Olan buccixator (Richardson) Wagler.

\section{Trumpeter swan.}

C'ygmus buccinator Rich., in Swainson and Rich., Fauna BorealiAmer., 1S31, vol. 2, p. 464. "Hudson's BAr." Audubon, Birds of Amer., 1843, rol. 6, p. 219, pls. 382, 383.

Fresh and salt water; nests on the ground.

Me.- Once erroneously recorded for $O$. cygmms.

N. H. Formerly a migrant (Belknap).

Mass. - Believed to have ocourred formerly as a migrant.

R. I., Coxs.- Doubtfully reported, and belicred to have occurred formerly ats a migrant.

HERODIONES.

IBIDES.

IBIDIDAE.

102. Gutra MlBA (Linné) Stejneger.

White Ibis.

Scolopax alba Limné, Syest. nat., ed. 10, 175s, vol. 1, p. 145. "AnERica."

Ibis alba (Limné). Audubon, Birds of Amer., 1\$43, vol. 6, p. 5 t, pl. 360 .

Swamps and lagoons; nests in trees and reedr marshes.

VT.-Aecidental visitor: South Woodstock, summer, 1S7S.

Coxx- - Acridental visitor: near Milford, May 23, 1875.

103. Pligadis a tomateis (Hasselquist) Stejueger.

\section{Glossy Ibis.}

Tringa antumnalis Hasselq., Reise nach Paläist., deutsche ausg., 1762, p. 306. [H,GYT.] 
HERODIONES.

IBIDES.

IBIDIDAE.

Ibis falcincllus Linné. Bonaparte, Amer. ornith., 1S33, vol. 4, p. 23 , pl. 23, fig. 1 .

Marshes and borders of lagoons; nests among low bushes or reeds.

N. H.- Accidental visitor: Alton, Oet. 18.58.

Mass. - Accidental visitor: noteworthy flights in May of 1850 and 1878 .

Conv.-Accidental visitor: Middletown, May 16, 1S50; Stratford (5 specimens) about 1836.

CICONIAE.

CICONIIDAE.

104. Mycteria americana Linné.

\section{Wood Ibis.}

Limné, Syrst. nat., ed. 10, 1758, vol. 1, p. 140. "Ix AuERica CALIDIORE."

Tantalus loculator Linné. Audubon, Birds of Amer., 1S43, vol. 6, p. 64 , pl. 361 .

Swamps and marshes; nests in trees.

Me.-Accidental visitor: Berwick, July 16, 1896.

N. H.- Erroneously recorded for Glossy ibis.

VT.-Accidental visitor: pair in summer, about 1897, near Burlington.

Miss.-Accidental visitor: (? Georgetown, June 19, 1850); Seekonk, July 17, 1896.

R. I.-Accidental visitor: Barrington, Aug. S, 1896. 
HEROIIONES.

HERODII.

ARDEIDAE.

105. Botaurus imatiginosus (Montagu) Stephens.

American bittern; Bog hen; Indian hen; Meadow hen; Stake driver; Thunder pump.

Ardea lentiginosa Montag., Ornith. dict., suppl., 1S13, [p. 1S6] "Her." "THE west of ExGland." Audubon, Birds of Amer., 1St3, vol. 6, p. 94, pl. 365. Egg, Cappen, 1SS6, pl. 19, fig. 14.

Fresh and salt marshes and borders of streams; nests on ground.

ME.- Uncommon migrant and summer resident. April 27Oct. 2S. Eggs, late May-June 6.

N. H.- Uncommon migrant and summer resident. Mar.-Nor. (Dec. 11). Eggs, May 27.

VT.- L'ncommon migrant and summer resident. April 30-fall.

MAss.-Common migrant and less common summer resident; easual in winter. Mar. 31-Nor. 26 (Dec. 30; Jan. 5). Eggs, May 5-.June 10.

R. I.-Common migrant and uncommon summer resident. April 6-Nov. 10. Eggs, May 23-June 13.

Coxs.-Common migrant and uncommon summer resident. April-Nov.

106. Ixobrychus exuls (Gmelin) A. O. U. comu.

\section{Least bittern.}

Ardea exilis Gmel., Sist. nat., 1785, rol. 1, pt. 2, p. 645. "JAmaiCA." Audubon, Birds of Amer., 18t3, vol. 6, p. 100, pl. 366. Egg, Capen, 1SS6, pl. 19, fig. 15.

Fresh malshes and swamps; nests among rushes or in bushes.

ME.- Rare summer resident in southem counties. (Mar. 4, 1S91, Georgetomn) May 12-Aug. (Nov. 13).

N. H.- Rare in summer; may breed in southeastern part: Hampton; Hampton Beach, 1S69; Seabrook.

VT.- Said to have occurred "formerly." 
HERODIONES.

HERODII.

ARDEIDAE.

Mass.- Rare local summer resident, mainly near the coast. April 15-Sept. 1. Eggs, June 1-29.

R. I.-Common summer resident; casual in winter. (Mar. 1)Sept. 14 (Feb. 2S, 1S81, Providence). Eggs, May 23-June 16.

Coxx.- Uncommon local summer resident. Spring-Sept. Eggs, June 10-27.

107. Ardea herodias Limné.

Great blue heron; Blue crane; Crane; Frog stabber; Poke.

Linné, Syrst. nat., ed. 10, 175\$, rol. 1, p. 143. "Avierica." Audubon, Birds of Amer., 1843, vol. 6, p. 122, pl. 369. Egg, Capen, 1S86, pl. 19, fig. 11 .

Swamps and borders of ponds; nests in trees.

Me.-Common migrant and local summer resident. Mar. 29Nor. 12 (Dec. 9). Egggs, late April-May 16.

N. H.-Common migrant and, northward from I. Winnepesaukee, a local summer resident. April 5-Nor. (Dec. 21; Dec. 31, 1901, Randolph).

VT.-Common migrant and, in northeru part, a local summer resident. Summer-(Dec. 22, 18st, Milton).

NAss.-Common migrant and rare winter resident; occasional in summer but not now known to breed (formerly at Nanshon). Mar. 15-June 4; (summer); July 30-Dee. 20 (winter).

R. I.-Common migrant. Mar,-May 4; July 15-Dec. 11.

Coxx.-Common migrant, occasional in summer; rare winter resident. Mar. 15-May (June 2); Aug.-Dec. (winter).

10S. Heronias egretta (Gmelin) Cabanis.

\section{American egret.}

Ardea egretta Gmel., Sist. nat., 17SS, vol. 1, pt. 2, p. 629. "IN

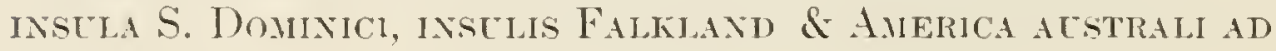


HERODIONES.

HERODII.

ARDEIDAE.

Lousianan." Aulubon, Birls of Amer., 1843, vol. 6, p. 132, pl. 370 .

Swamps and lowlands; nests in small trees.

Me.-Occasional summer visitor. April 7 -Aug. 22.

N. H.- Rare summer visitor: near Newmarket, 1897 .

TT.-Rare summer visitor: New Haven, two in Aug. 1852; Winooski River, summer, 1869.

Mess.-Oceasional summer and fall risitor. April-Nor. 22.

R. I.-Occasional summer visitor; may have bred at Wesquage Pond in 1894. June 1-Oct. 12.

Coxx.- Oecasional summer risitor. July-Oct.

109. Egretta caxdidsana (Gmelin) Gosse.

\section{Snowy heron.}

Ardea candidissima Cimel., Syst. nat., 17Ss, vol. 1, pt. 2, p. 633. "In mexdats prope Carthagenat Americale." Audubon, Birds of Amer., 18t3, vol. 6, p. 163, pl. 374.

Swamps and lowlands; nests in smill trees.

ME.- Erroneously accredited.

VT.-Aecidental visitor: St. Albans Bay, two in Oct., 1890.

MAss-Accidental visitor: near Boston, 1862; Nantucket, March, 18S1; Northampton, 1SS7 (? nee Essex Co.).

Coxs-- Accidental visitor: Hartford; Stratford ("seen").

110. Frorida camblea (limé) Baird.

\section{Little blue heron.}

Ardea caerulea Linué, Syst. nat., ed. 10, 1758, vol. 1, p. 143. "1x America septentrionall." Audubon, Birds of Amer., 1St3, vol. 6, p. 14S, pl. 372 .

Swamps and lowlands; nests in busles. 
HERODIONES.

HERODII.

ARDEIDAE.

Me.- Aecidental visitor. April 1-Sept.

N. H.-Aceidental visitor: Amherst, April 2S, 1897.

TT.-Doubtfully recorded.

Mass.- Occasional visitor. April 14-Aug

R. I.- Rare visitor. June 7-Aug.

Covx.- Rare visitor. May-Sept. 7.

111. Butorides virescens (Limné) Bonaparte.

\section{Little green heron; Fly-up-the-creek; Green heron; Poke.}

Ardea virescens Linné, Syst. nat., ed. 10, 175S, rol. 1, p. 144. "Airerica." Audubon, Birds of Amer., 1S43, vol. 6, p. 105, pl. 367. Egg, Capen, 18s6, pl. 19, fig. 12.

Mainly freshwater streams and ponds; nests in small trees.

ME.- Incommon migrant and summer resident in southern counties. May 2-Sept.19.

N. H.- Uncommon summer resident in southern half, and in Connecticut valley. Late April-Oct. Eggs, June 16.

rT.- Incommon summer resident in southern part. MayOct.

MAss-C Common summer resident. (April 9 and 16) April 26Oct. 16. Eggs, May 10-June 2.

R. I.-Common summer resident. April 20-Sept. 24. Eggs, May 21, 23.

Coxx-Common summer resident. Iate April-Oct. Eggos, May $30-$ June 17.

112. Nycticorax xicticorax Nafrics (Boddaert) Zeledon.

Black-crowned night heron; Buttermunk; Qua-bird; Quok; Shitepoke; Squawk.

Ardea naeria Bodd., 'Tabl. d. planch. enlum. d'hist. nat., 1783, p. 56. Based on Buffon: "CaYexie." 
ARDEIDAE.

Ardea nycticorax Iinné. Audubon, Birels of Amer., 1543, vol. 6, p. S2, pl. 363. Eigg, Capen, 1SS6, pl. 19, fig. 13.

Swamps and marshes; nests in colonies in trees, rarely on the ground.

ME.- Common summer resident, chicfly near the coast. SpringSept. 16. Egge, May 26.

N. H.- Uncommon summer resident north to southeru White MIt. valleys. April-Oct.

TT.- Uncommon summer resident near lakes and streams.

Mass. - Common migrant and summer resident; rare winter resident near coast. Mar. 27-Nov. 1 (winter). Eggs, May 2June 13.

R. I.-Common summer and rare winter resident. April 1Sept. 23 (winter). Eggs, May 7-June 7.

Coxs.-Common migrant and less common summer resident. April-Oct. Eggs, April 17.

113. Nyctanassa violacea (Limé) Sluarpe.

\section{Yellow-crowned night heron.}

Ardea violacea Iinné, Syst. nat., ed. 10, 175S, vol. 1, p. 143. "In America septentrional.," Audubon, Birds of Amer., 1St3, vol. 6, p. 89, pl. 364.

Swamps and lowlands; nests in low trees.

Me.-Accidental visitor: 1)eering, April 13, 1901; Portand. April 11, 1906 .

MAss. - Accidental visitor: six instances. July-Oct.

R. I.-Accidental visitor: Newport, Aug. 1S92; 'liverton, April 23, 1886. 
PALUDICOLAE.

GRUES.

GRUIDAE.

114. Grus anericana (Limné) Vieillot.

\section{Whooping crane.}

Audubon, Birds of Amer., 1842, rol. 5, p. 1SS, pl. 313.

Ardea americana Linné, Srst. nat., ed. 10, 175S, rol. 1, p. 142. "IN Anerica septeNtrionali."

Freshwater marshes and open lowlands; nests in marshes.

T., MAss. - Supposed to have been a migrant in colonial times.

115. Gres Caxadexsis (Limné) Temminck.

\section{Little brown crane.}

Ridgway, Manual of No. Amer. birds, 1S57, p. 135.

Ardea canadensis Linné, Syst. nat., ed. 10, 175S, rol. 1, P. 141. "IN AMERICA SEPTENTRIONALI."

Gins fraterculus Cassin. Baird, Cassin, and Lawrence, Expl. and surveys for railroad to Pacific, 1S5s, birds, pl. 37.

Grassy flats and marshes; nests on the ground.

R. I. - Accidental risitor: Natick Hill, Oct. 9, 1889.

116. Grós mexicaNa (Müller) Tieillot.

\section{Sandhill crane.}

Coues, Key to No. Amer. birds, ed. 5, 1903, rol. 2, p. StS.

Ardea (grus) mexieana Mïll., Limné's Vollstand. naturșrst., suppl., 1776, p. 110. "МЕxico."

Grus amoricana Forst. Audubon, Birds of Amer., 1842, rol. 5, pl. 314.

Freshwater marshes and open lowlands; nests in marshes.

․ H.- Believed to have occurred formerly; now accidental visitor: Wakefield, 1896 or 1897 .

VT.-Believed to have occurred formerly as a migrant; now accidental visitor: Lunenburg.

Mass., Cors.- Believed to have occurred formerly as a migrant. 
PALUIICOL.AE.

RAILI.

RAILIDAE.

117. Ratucs EleGixs Aulubon.

King rail; Fresh-marsh hen; Royal rail.

Audubon, Ornith. biogr., 1835, vol. 3, 1. 27, pl. 203. "Siot"Trerx States." Coues, Key No. Aner. birds, ed. 5, 190:3, vol. 2, p. 854 .

Freshwater marshes; nests on the gromud.

ME.-Oenasional fall visitor. Selt. 19-Dere. 17. ,

Mass. Oceasional risitor at all seasons; may breed.

R. I.--Rarle visitor (four reeorls). Jatr., Feb., Marr., fall.

Coxx.- Rare summer resident (Sirbrook, Surtford) and occasional at other scatsons.

118. Raldis crepitans Gmelin.

\section{Clapper rail; Salt-marsh hen.}

Gmel., Syst. nat., 17SS, rol. 1, pt. 2, p. 713. " " Toreboraco." Audubon, Birds of Amer., 18t2, vol. 5, p. 16.5, pl.:310. Eger, Capen, 1S\$6, pl. 20, figs. 1, 2.

Saltwater marshes; nests on the ground.

Me.-Accidental visitor in southern countics: Popham Beach, Oct. 12 and (25), 1900; Sabattus Pond, 1S74; ? York and Cumberland Cos.

․ H.-Accidental visitor: Portsmouth.

VT.-Aceidental visitor: Burlington (Conger).

Mass.- Occatsional visitor, maly breed.

R. I.-Doubtfully recorded.

Coxs- Rare visitor and summer resident (New Haren Stratford).

119. Ratues virainaxis Limné.

\section{Virginia rail; Water-hen.}

Limné, Syst. nat., ed. 12. 1766, rol. 1. p. 2063. "Is America 
RALLIDAE.

septentrionili." Audubon, Birls of Amer., 1S42, vol. 5, p. 174, pl. 311. Egg, Capen, 1S\$6, pl. 20, figs. 3, 4.

Fresh and brackish marshes; nests on the ground.

Me.- Rare summer resident. Late April-Oet.

. H. - Rare summer resident south of White Mts. AprilOet. 1. Egges, May 21-2S.

TT.- Rare summer resident locally: Burlington; Rutland Co.

Mass.-Summer resident, more common near coast; rare winter resident (Cape Cod). April 11-Nov. 27 (Jan. 1, 1891, Worcester) (winter). Leggs, May 12-June 1.

R. I.-Common migrant and summer resident. April-Nor. 2. Eggs, May 29.

Coxs.- Common migrant and summer resident. Eggs, June 6.

120. Porzana carolina (Limné) Baird.

\section{Sora; Carolina rail; Meadow-hen; Rail-bird.}

Rallus carolimus Limné, Syst. nat., ed. 10, 175s, vol. 1, p. 153. "IN Ainerica septentrionalı."

Ortygometra carolinus (Linné). Audubon, Birds of Amer., 1S42, vol. 5, p. 145, pl. 306. Egg, Capen, 1S\$6, pl. 20, fig. 5.

Fresh and brackish marshes; nests on the ground.

ME.- Uncommon summer resident. Late April-Oet. 26. Eggs, -.July 27.

N. I.- Uncommon summer resident northward to White Mt. ralleys. May-Oct.

VT.- Uncommon summer jesident.

Mass.-Common migrant and local summer resident. (Mar. 20) April 14-Nov. 1 (Dee. 20). Eggs, June 9-11.

R. I.-Common migrant and summer resident. (Mar. 2) April-Nov. 10. Egggs, Mą 24.

Cons.-Common migrant and summer resident. April-Oct. (Dec. 29, 1S\&1, Hartford). 
121. Coturnicops voveboracensis (Gmelin) Bonaparte.

\section{Yellow rail.}

Fuliea noveboracensis Gmel., Syst. nat., 17SS, vol. 1, pt. 2, p. 701. "IN Noveboraco."

Rallus noveboracensis (Lath.). Bonaparte, Amer. ornith., 1833, vol. 4, p. 136, pl. 27, fig. 2. Egg, Capen, 1S56, pl. 20, fig. 6.

Freshwater marshes; nests on the ground.

Me.- Rare migrant and said to have bred near Calais. May 31; Oct. 4-Nor. 15.

N. H.- Rare migrant: Hampton.

VT.-Rare migrant: Newport; northern Vt.; Rutland Co.

MAss. - Rare migrant and possible summer resident. AprilMay 26; Sept. 2-Oet. 22.

R. I.- Rare migrant (five records). Sept. 2 S.

Coxs.- Rare spring and uncommou fall migrant; said to have bred at Middletown, 1874, 1875. (MIar. 24, Garlordsrille); Sept.Oct. 14 (Nor. 10).

\section{Crecisces Jamaicensis (Gmelin) Cabanis.}

\section{Black rail; Little black rail.}

Rallus jamairensis Gmel., Syst. nat., 17SS, vol. 1, pt. 2, p. 71S. "Ja.talca."

Porzana jamaicmsis (Gmel.). Chapman, Handlb. birds east. No. Amer., 1S95, p. 144; Allen, Auk, 1900, vol. 17̄, pl. 1.

Freshwater marshes; nests on the ground.

ME-Doubtfully recorded from Scarborough, Oct. 4, IS\&1.

MAss.- Very rare visitor and summer resident (at Chatham, Hazardville). May 16-Sept. 20. Eggrs, May.

Cons.-Very rare summer resident (at Lyme, Sarbrook); also taken at Hazenville. Eggos, June 6-July 10. 
PALLDICOLAE.

R.ALLI.

RALLIDAE.

123. Crex crex (Linné) Sharpe.

Corn crake; Daker-hen; European land rail.

Coues, Key to No. Amer. birds, ed. 5, 1903, vol. 2, p. 859.

Rallus crex Limné, Srst. nat., ed. 10, 1758, vol. 1, p. 153. "Ix EUROPAE AGRIS, CARECTIS.".

Crex pratensis Bechst. Dresser, Birds of Europe, 1871-81, rol. 7 , pl. [499]. Egg, Seebohm, 1896, p. \$3, pl. 22, fig. 9.

Grassy meadows and grain fields; nests on the ground.

Me.-Accidental from Europe: near Falmouth, Oct. 14, 1 s 9.

R. I.-Accidental visitor: Cranston, 1857.

Coxx.-Accidental risitor: Saybrook, Oct. 20, 1857.

124. Ionornis Martinica (Linné) Reichenbach.

\section{Purple gallinule.}

Fulica martiniea Linné, Syst. nat., ed. 12, 1766, rol. 1, p. 259. "in Martinicae inuNdatis."

Gallinuln martinica (Limé). Audubon, Birds of Amer., 1St2, vol. 5, p. 128, pl. 303.

Fireshwater marshes; nests among rushes.

ME.-Accidental visitor. April 11-1)ec. 17.

N. H.-Accidental risitor: Dover; Rre.

T. T. Doubtfully recorded.

MAss.-Occasional visitor, mainly in April and Oct.-Nor., once in June.

R. I.- Occasional visitor. Jan.; MIay; June S, 1902, Seaconnet Point; Aug.; fall.

Coxx.- Rare visitor: Middletown, 18.5.5.

125. Gadixela galeata (Lichtenstein) Bonaparte.

Florida gallinule; Pond hen; Red-billed mud-hen.

Crex galeata Licht., Verz. d. doubletten zool. mus. Berlin, 1S23, p. So. "Sax Palio," Brazil. 
RALLIDAE.

Gallinula chloropus Linné. Audubon, Birds of Amer., 1842, vol. 5, p. 132, pl. 304. Egg, Capen, 1S\$6, pl. 20, figs. 7, S.

Freshwater marshes; nests among rushes near the ground.

Me.- Rare migrant and (?) summer resident. May 5; Sept. 20-Oct. 16.

N. H.-Accidental visitor: Rollinsford.

VT.- Rare local summer resident at Lake Bomoseen, Lake Champlain, St. Albans. April 28-fall. Egggs, May 2S-June 5.

MAss. - Rare local summer resident, mainly near coast. April 29-Oct. 25 (Nov. 9). Eggs, June 5-July 17.

R. I.-Uncommon local summer resident. May-Nov. 29.

Cons.- Uncommon local summer resident. Eggs, June 25 (Stratford).

126. Fulica americana Gmelin.

\section{American coot; Blue Peter; Coot; Marsh hen; Mud-hen; Pond crow; Pond hen.}

Gmel., Syst. nat., 17SS, vol. 1, pt. 2, p. 704. "1N AMERICA Septextrionali." Audubon, Birds of Amer., 1S+2, vol. 5, p. 13S, pl. 305. Egg, Capen, 18\$6, pl. 20, fig. 9.

Freshwater ponds and marshes mainly; nests among reeds.

Me.- Uncommon migrant, mainly in fall. (Mar. 23) April 14May 13; Oct. 26-Nor.

N. H.- Uneommon migrant, mainly in southern part. Spring; late Aug.-Oct. 15.

VT.- Lncommon migrant and rare summer resident (Lake Bomoseen).

MAss.- Rare spring and uncommon fall migrant; rare winter resident near coast; may breed rarely (pair, Cheshire reservoir, June 21, 1S92). Mar. 29-April 25; (June 21); (Aug. 16) Sept. 3 -Nov. (winter). 
PALCDICOLAE.

RALLI.

RALLIDAE.

R. I.- Common migrant, mainly in fall. April-May 2s; Sept. 26-Dec. 20.

Cors.-Common migrant, mainly in fall. Spring; Sept. 29Nov. 14 .

LIIICOLAE.

PHALAROPODIDAE.

127. Phalaropls fulicarics (Linné) Bonaparte.

Red phalarope; Bank-bird; Brown bank-bird; Gray phalarope; Gulf-bird; Sea goose; Whale-bird.

Audubon, Birds of Amer., 1842, vol. 5, p. 291, pl. 339. Egg, Capen, 1886 , pl. 18, fig. 11 ; pl. 19, fig. 1.

Tringa fnlicaria Linné, Syst. nat., ed. 10, 175s, vol. 1, p. 14 S. "Alierica."

Chiefly maritime; nests on the ground in marshes.

XIE.- L Lncommon migrant, mainly offishore, and rare summer resiclent (near Calais). May \&-17; (summer); July 25-Oct. 17.

N. H.- Migrant, mainly off'shore; sometimes common. May; Oct.

VT.- Rare migrant at Lake Champlain.

MAss.-Offishore migrant, irregularly common. May 1-26; Aug. 31-Nor. 24.

R. I., Cox:- Offshore migrant, sometimes common. Late May; Sept. 20-Dec, 20.

12S. Lomipes lobatus (Limné) Baird, Brewer, \& Ridgray.

Northern phalarope; Bank-bird; Jersey goose; Mackerel goose; Red-necked phalarope; Sea goose; Sea snipe; Whalebird; White bank-bird.

Tringa lobata Linné, Sirst. nat., exl. 10, 175\$, vol. 1, pp. 14\$, \&24. "Is Anerica septentrionali, Lappoxia."

Lolfipes hyperloreus Lath. Audubon, Bircls of Amer., 1St?, vol. 5, p. 29.5. pl. 340. Egg, Seebohm, 1896, p. 131, pl. 39, fig. 1.

Chiefly naritime; nests on the ground in mirshes. 
AVES.

LIJICOLAE.

\section{PHALAROPODIDAE.}

Me.-Common migrant, mainly offshore. (Feb.) May 3Jume 15; July 2t-Nor.

X. H.-Common migrant, mainly offshore. May; Aug. 9Sept. 20.2.

Mass- Common migrant, mainly offshore. May 1-26; Aug. $10-$ Oct. 13 .

R. I.-Common migrant, mainly offshore. May; Aug. 16Sept. 15.

Conx.- (offshore migrant. Spring?; Aug. 4-Sept. 27 (Dec. 25).

129. Stegaxopes tricolor Vieillot.

\section{Wilson's phalarope.}

Vicill., Nour. dict. d'hist. mat., 1819, vol. 32, p. 136. "PARAGCAY."

Phalaropus witsonii Salnine. Swainson and Richardson, Fanna Boreali-Amer., 1831, rol. 2, p. 40.5, pl. 69.

Chiefly freshwater marshes; nests on the ground.

Mls-Accidental visitor: Sabattus Pond, Sept. or Oct. 1906; Scarborough, three, June 9, 1891.

N. Il.-Arcidental visitor: Rye Beach, Aug. 15, 1872.

Mass.-Accidental visitor (five records). May 20; Aug. $1 S-31$.

R. I.- Acriclentall (fall) visitor (five records). Aug. 2-Sept. 13.

Coxw-A - Aceidental risitor: Bridgeport.

\section{RECURVIROSTRIDAE.}

130. Recurthostra americana fomelin.

\section{American avocet.}

Guncl., Syst. mat., 178S, vol. 1, pt. 2, p. (993. "Ix America

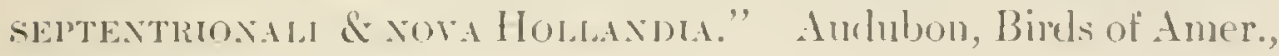
18t3, vol. 6, ]. 2- t, pl. 35.3. 


\section{LIMICOLAE}

\section{RECURVIROSTRIDAE.}

Open lowlands and marshes; nests on the ground.

Me.-Accidental visitor: Cape Elizabeth, Nov. 5, 1878.

VT.-Accidental visitor: St. Albans, fall, 1S75, and 1590; (nec Rutland, 1SS2).

Mass.-Accidental visitor: Ipswich, three, Sept. 13, 1S96; Lrmn; Natick, Oct. 19, 1850.

Coxx.-Accidental visitor: between Sarbrook and East Lyme, 1871.

131. Hinaxtopus aiexicanus (Müller) Ord.

\section{Black-necked stilt.}

Charadrius mexicanus Müll., Linné's Vollstand. natursystst, suppl., 1776, p. 117. "МLexico."

Himantopus nigricollis Vieill. Audubon, Birds of Amer., 1843, vol. 6, p. 31, pl. 354 .

Salt marshes and lagoons; nests on the ground.

Me.-Accidental risitor: Rockland, Mar, 1859; (nec Boardman, New Brunswick).

N. II.-Accidental visitor: Rye Beach.

Mass.-Aecidental risitor: Lynn; Mass. coast.

\section{SCOLOPACIDAE.}

132. Philohela ainor (Gmelin) Gray.

\section{American woodcock; Bog-sucker; Woodcock.}

Scolopax minor Gmel., Syst. nat., 17SS, rol. 1, pt. 2, p. 661. "ix Anericae, a Septembri inde in Carolinae, ab Aprilis FINE IN NoVERORACI SILVIS HUMIDIS."

Wicroptera americana Audubon, Birds of Amer., 1St3, rol. 6, p. 15, pl. 352. Eggg, Capen, 1S86, pl. 19, fig. 2.

Freshwater swamps, damp woods and thickets; nests on the ground. 
LIMICOLAE.

\section{SCOLOPACIDAE.}

Me.- Common migrant and summer resilent; possibly winters. (Mar. 10) Mar. 23- Vov. 30 (Dec. 18) (Feh., two instances). Eggs, April 26-\Гаy 19.

N. H.- Uncommon migrant and summer resident. Late Mar.-Oet. Egges, Maý 10.

VT.- Uneommon migrant and summer resident. Mar. 27-Oct. Egges, Ma! $y^{r} 4$.

MAss. - Common migrant and uncommon summer resident; rare in winter. Mar. t-Nov. 25 (winter). Eggs, April 11-May 6.

R. I.-Common migrant and now a rare summer resident. Mar.-Dec. 4. Eggs, April 5-MIay 17.

Cons.- Common migrant, uncommon summer and winter resident. Feb. 26-Nor.; winter. Eggs, (Mar. 30) April 3June 3; July 20 (2d brool).

133. Galdinago delicata (Orl) A. O. U. check-list.

\section{Wilson's snipe; Alewife-bird; Common snipe; English snipe; Jack snipe.}

Scolopax delicata Ord, Wilson's Ornith., 1525, vol. 9, p. cexiin. "KentuckY, . . . llunois, . . Peñsylyania."

Scolopax wilsonii Temm. Audubon, Birds of Amer., 1St2, vol. 5, p. 339, pl. 350. Egg, Capen, 1S86, pl. 19, fig. 3.

Fresh and brackish meadows and marshes; nests on the gromnd.

ME.-Common migrant and rare summer resident. April 19May 15; summer; Sept.-Nor. 15.

N. H.- Common migrant, mainly coastwise; very rare winter resident (Nashua). Mar--May 7 ; July 22-micl-Dec. (winter).

VT.- Uncommon migrant and rare winter resident (Rutland Co.); ?"summer resident."

MAss.- Common migrant, rare winter resident; rare and irregular summer resident (at Carlisle, Concord). (Mar. S) Mar. 20-Nay 1S; (summer); Aug. 1-Nov. 30 (winter). 
LIMICOLAE.

SCOLOPACIDAE.

R. I.- Common migrant and rare winter resident. Mar. 9May 6; (July 29) Aug. 12-Nor. 14 (Dec., Jan.).

Coxx-Common migrant, rare summer and winter resident. Mar. 1S-Dec.; minter. Eggs, May 13 (Portland).

134. Macrorhayphes Griseus (Gmelin) Leach.

Dowitcher; Brownback; Deutscher; Fool plover; German snipe; Grayback; Gray snipe; Red-breasted snipe; Robin snipe.

Scolopax grisca Gmel., Sist. nat., 17SS, vol. 1, pt. 2, p. 6.5S. "IX Noteboraci inaritiniss."

Scolopax noveboracensis Gmel. Audubon, Birds of Amer., 1S43, vol. 6, p. 10, pl. 351. Egg, Seebohm, 1896, p. 143, pl. 41, fig. $S$.

Chiefly maritime marshes and mudflats; nests on the ground.

ME.-Common migrant coastwise. Mą 21-29; late JulySept. (Oct. 29).

N. H.- Migrant, common in fall. May 20-25; July 20-Sept.

MAss. - Rare spring and uncommon fall migrant, mainly coastwise. Mar 1-June 7 (.June 1S); July 5-Sept. 30.

R. I.- Lncommon migrant coastwise. Maý; July 5-Oet. 20.

Coxx- - Encommon migrant, mainly in fall.

135. MACRoRHAMPHL's SCOLOPACEL's (Say') Iamence.

\section{Long-billed dowitcher.}

Elliot, New and unfigured birds No. Amer., 1869, rol. 2, pl. 40.

Limosa scolopacea Say, Long's exped. to Rockir Mts., 1S23, rol. 1, p. 170. "NEAR THE BoWTer CREeK," IoWA.

Chiefly maritime marshes and mudflats; nests on the ground.

Mass. - Rare fall migrant. Aug. 29-Nor. 3.

R. I.- Rare fall migrant: Middletown, Oct. S, $1 S 90$. 
LIMICOL.AE.

SCOLOPACIDAE.

136. Micropalama mimantopes (Bonaparte) Baird.

Stilt sandpiper; Bastard yellow-leg; Long-legged sandpiper; Mongrel.

Tringa limantopus Bonap., Ann. lyceum nat. list. New York, 1826, vol. 2, p. 157. "Loxg Brixch, New Jerser." Bonip., Amer. ornitl., 1\$33, vol. 4, p. 89, pl. 2.5, fig. 3.

Marshes and mulflats; nests on the ground.

ME.- Rare fall migrant coastwise. July 19-Sept. 26 (Oct. 2, 190s, Pine Point).

X. H.- Rase fall unigrant coastwise. July 31-Aug. 2S.

Mass- Lucommon fill migrant coastrise. July 19-Sept. 29.

R. I.- Very rare spring and uncommon fall migrant. May 9, 189.5, Sakonnet Point; July 6-Sept. 19.

Coxx.- Rare migrant: Nerr Haven, Sept. 16, 1886.

137. Tringa cantest Limné.

Knot; Ash-colored sandpiper; Blue plover; Grayback; Gray snipe; Red-breasted plover or sandpiper; Robin snipe; Silver plover.

Linné, Srrst. nat., ed. 10, 175s, vol. 1, p. 149. "ri Europı." Egge, scebohm, 1896, p. 14t, pl. 43, fig. 10.

Tringa islandica Limné. Audubon, Birds of Amer., 1St2, rol. 5, p. 254, pl. 328 .

Sandy beaches; nests on the ground.

Me., X. II.- Lncommon migrant coustwise. Miy 24-June 11; Aug.-Sejt.

Mass.-Common migrant coastwise, casmal inland (Spring field); rare winter resillent. May 11-June 5 (June 25); July 13-Nor. S (winter).

R. I.- Common migrant constwise. May; July 11-Sept. 14.

Coxx--Common migrant coastwise. 
LIMIICOLAE.

SCOLOPACIDAE.

138. Arquatella maritima (Brünnich) Coues.

\section{Purple sandpiper; Rock snipe; Winter peep; Winter snipe.}

Tringa maritima Brünn., Ornith. borealis, 1764, p. 54. "CHristiaxsöe" I., Denmark, aNd "Norvegla." Audubon, Birds of Amer., 1842, vol. 5, p. 261, pl. 330. Egg, Seebohm, 1896, p. 146, pl. 43 , figs. 2,5 .

Rocky islands and coasts of the sea; nests on the ground.

ME.-Common migrant and winter resident coastwise; accidental in summer. Sept. 15-April (Aug. 6, 1907, Metinic Green Id.; Aug. 11, 1902, Metinic reef).

N. H.- Rare winter visitor coastwise.

$V_{T}$ - Doubtfully recorded.

Mass.-Common fall and winter visitor coastwise. (July 30) Sept. 6-April 19 (May 11).

R. I.-Common winter resident on rocky coasts. Sept. 13April 15.

Coxv.- Common winter resident on rocky islands coastwise.

139. Pisobia maculata (Vieillot) A. O. U. comm.

Pectoral sandpiper; Brown-back; Creaker or Krieker; Grassbird; Grass snipe; Jack snipe; Pert; Squatter.

Tringa maculata Vieill., Nour. dict. d'hist. nat., 1819, vol. 34, p. 465. "aux Îles ANTilles et dans les parties aí́ridionales des Etats-Unis."

Tringa pectoralis Bonap. Audubon, Birds of Amer., 1842, vol. 5, p. 259, pl. 329 . Egg, Seebohm, 1896, p. 148, pl. 43, fig. 12.

Marshes and inudflats; nests on the ground.

Me.- Rare spring and common fall migrant, mainly coastwise. (Spring); Aug. 13-Oct. 5.

N. H.- Migrant, common in fall, mainly coastwise. July-Oct. 10. 
LIMICOLAE.

SCOLOPACIDAE.

VT.- Uncommon fall migrant.

Mass. - Rare spring and common fall migrant. (April 4) April 9-May 16 (May 23); July 15-Nov. 11.

R. I.- Rare spring and common fall migrant. April 25; July 16-Oct. 20.

Cons. - Rare spring and common fall migrant.

140. Pisobia fuscicollis (Tieillot) A. O. U. comm.

White-rumped sandpiper; Bonaparte's sandpiper; Bull-peep; Schinz's sandpiper.

Tringa fuscicollis Vieill., Nouv. dict. d'hist. nat., 1819, vol. 34, p. 461. "Paraguar." Egg, Seebohm, 1896, p. 145, pl. 43, fig. S. Actodromas fuscicollis (Vieill.). Baird, Brewer, and Ridgway, Water birds of No. Amer., 1SSt, rol. 1, p. 227, fig.

Tringa schinzii Brehm. Audubon, Ornith. biogr., 1835, rol. 3, pl. $27 \mathrm{~S}$.

Beaches and marshy shores; nests on the ground.

Me.-Uncommon migrant, mainly coastwise. Nay 29, 30; late July-Oct. 23 (Nor. 1S).

N. H.- Uncommon migrant, mainly coastwise. ? Spring; JulyOct. 14.

VT.- Uncommon fall migrant. Aug.-Sept.

MAss. - Rare spring and common fall migrant mainly coastwise. May 15-June 5; July 10-Nov. 10.

R. I.- Uncommon fall migrant. July 11-Oct.

Coxv.- Uncommon migrant. Aug. 31.

141. Pisobla bairdil (Coues) A. O. U. comm.

Baird's sandpiper.

Actodromas bairdii Cones, Proc. acarl. nat. sci. Phila., 1\$61, p. 194. "North America." Baird, Brewer, and Ridgway, Water birds No. Amer., 18S4, vol. 1, p. 230, colored fig. of head. 
LIMICOLAE.

$$
\text { SCOLOPACIDAE. }
$$

Beaches and marshes; nests on the gromnd.

ME.- Rare fall migrant. Aug. 7-Sept. 20 (Nor. 1, 1SS1, Bangor).

\. H.- Rare fall migrant. Aug. 26-Sept 5.

Mass.- Rare fall migrant. July 27-Oct. 1.

R. I.- Rare fall migrant (seren records). Aug. 26-Sept. 17.

Cons.- Rare fall migrant: New Haven, Oct. 19, 18s9, Oct. 2S, 1SS7; Stratford, Nor. 3, 1 SSS.

142. Pisobia minutilla (Vieillot) A. O. U. comm.

Least sandpiper; Bumble bee; Meadow ox-eye; Mud peep; Peep; Wilson's sandpiper.

Tringa minutilla Vieill., Nouv. dict. d'hist. nat., 1\$19, vol. 34, p. 466. "EN AMérique Jusqu'AU delà du CANAdA." Egg, Seebohm, 1896, p. 149, pl. 4t, fig. S.

Tringa pusilla Wils. Audubon, Birds of Amer., 1S 42 , rol. 5, p. 280 , pl. 337 .

Marshes and mudflats; nests on the ground.

Me.-Common migrant. May 13-2t (June); July 5-Oct. 1 (Oct. 15).

\. H.-Common migrant. Late May-June 3; July 10-Sept.

VT.-Uncommon migrant.

MAss.-Common migrant. (April 18) May 5-June 7; (summer) July 6-Sept. 21 (Oct. 13).

R. I.-Common migrant. (April 25)-May 26; July 15-Oct.

Coxs.-Common migrant. May-Jme; Aug.-Sept.

143. Pelidna alpina (Linné) C. L. Brehm.

\section{European dunlin.}

Sharpe, Cat. birds British mus., 1896, vol. 24, p. 602. Dresser, Birds of Europe, 1s71-\$1, rol. S, p. [21], pl. [5ts]. 
LIMICOLAE.

\section{SCOLOPACIDAE.}

Tringa alpina Limné, Syst. nat., ed. 10, 1758, vol. 1, p. 149. "1. LAPPONı." Egg, Seebohm, 1S96, p. 1H, pl. 43, figs. 7, 9.

Beaches and marshes; nests on the gronnd.

Mass.-Accidental from Old World: Chathan, Aug. 11, 1900.

144. Pelmoxa alpixa sakmalisa (Tieillot) Buturlin.

American dunlin; Black-bellied sandpiper; Black-breast; Brant-bird; Crooked-billed snipe; Fall snipe; Lead-back; Ox-bird; Ox-eye; Red-back; Red-backed sandpiper.

Scolopax salihalina Vieill., Nour. dict. d'hist. nat., 1\$16, vol. 3, p. 359. "Ex Russie."

Tringa alpina Linné. Audubon, Birds of Amer., 1S 42 , vol. 5, p. 266, pl. 332 .

Beaches and marshes; nests on the ground.

Me.- Rare spring and commoner fall migrant coastwise Late May; Oct.-Nor. 17.

N. H.- Uncommon coastwise migrant.

MAss. - Rare spring and common fall migrant coastwise. (? Mar. 1.5) late April-May 20 (.June 18); Sept. 1-Dec. 13 (Dee. $24)$.

R. I.- Rare spring and common fall migrant coastwise. May"; Aug. 29-Oct. 29.

Coxx.- Rare spring and common fall migrant; "may winter."

145. Eroda rerreginea (Brümich) A. O. U. comm.

\section{Curlew sandpiper.}

Tringa ferruginea Brünn., Omitlı. bor., 1764, p. 53. "Istaxnda \& Christiansöre" Islant.

Tringa subarquata 'Temm. Audubon, Birds of Aner., 1St?, rol. 5, p. 269, pl. 333.

Seacoasts and tundras; nests on the ground. 
LIMICOLAE.

\section{SCOLOPACIDAE.}

Me.-Accidental visitor: Pine Point, Cumberland Co., Sept. 15, 1881 .

Mass.-Accidental visitor: five records. May; Aug.

R. I.- Doubtfully recorded.

Corr.-Accidental visitor: four records. (June 1874, New Haven); Aug. 30-Oct. 3.

\section{Ereunetes pusillus (Linné) Cassin.}

\section{Semipalmated sandpiper; Black-legged peep; Peep; Sand} ox-eye; Sand peep.

Tringa pusilla Linné, Syst. nat., ed. 12, 1766, vol. 1, p. 252. "in Doningo."

Tringa semipalmata Wils. Audubon, Birds of Amer., 1842, vol. 5, p. 277, pl. 336 .

Sand beaches and marshes; nests on the ground.

Me.-Common migrant. May-June 5; July 29-Sept. 28 (Oct.).

N. H.-Common migrant. Nay; July-early Sept.

VT.-Uncommon migrant to larger lakes.

MASS.- Common migrant; non-breeding birds occur in summer coastwise. (May 1) May 13-June 14; (summer); July 3-Oct. S (Oct. 30).

R. I.-Common migrant. May 13-June 2; July 14-Oct. 10.

Cosx.-Common migrant. May-early June; July-Oct.

\section{Ereunetes mauri Cabanis.}

\section{Western semipalmated sandpiper}

Cab., Journ. f. ornith., 1856, p. 419. "CuBA."

Ereunetes pusillus occidentalis (Lawrence). Baird, Brewer, and Ridgway, Water birds of No. Amer., 1854, vol. 1, p. 206, fig. of head.

Sand beaches and marshes; nests on the ground. 


\section{LIMICOLAE.}

\section{SCOLOPACIDAE.}

N. H.- Rare fall migrant: Hampton, Oct. 10, 1899 .

Mass.- Uncommon fall migrant. July 19-Sept. 20.

R. I.-- Rare fall migrant: Middletown, Aug. 25, and Aug. 29, 1899 .

Conv.- Ratre fall migrant: Lyme, Sept. 4, $18 S 9$ (six).

14S. Calidris leucophate (Pallas) A. O. U. comm.

Sanderling; Beach-bird; Beach plover; Bull-peep; Grayback; Ruddy plover; Surf snipe; Whitey; Whiting.

Tringa leueophaca Pallas, in Troeg's Catalogue, 1764, p. 32. North Sea const.

Tringa arenaria Bonap. Audubon, Birds of Amer., 1St2, rol. 5, p. 287, pl. 33S. Egg, Seebohm, 1896, p. 150, pl. 41, fig. 4.

Sand beaches; nests on the ground.

ME., N. H.- Uncommon spring and common fall migrant coastwise. Nay 5-30; late July-Oct. 13 (Nov. 5, Portland).

VT.- Rare migrant. Sept.

Mass.- Common coastwise migrant and, south of Cape Cod, a winter resident. (Late April) May 19-June 4 (June 24); July 6-Dec. 6 (Dec. 13); winter.

R. I.-Common migrant, casual inland; probably a tare winter resident. (Mar. 20) May-June; Aug. 1-Nov. 5.

Coxx-C Common migrant coastwise. May; July-Oct.

149. Linosa fedoa (Linné) Sabine.

Marbled godwit; Brown marlin; Red curlew.

Audubon, Birds of Amer., 1S42, vol. 5, p. 331, pl. 34 .

Scolopax fodoa Linné, Syst. nat., ed. 10, 175S, vol. 1, p. 146. "IN America septentrionala."

Beaches and marshes; nests on the ground.

Me.-- Rare migrant. May, $18 \$ 4$ (Searborough); Aug. SSept. 13. 
LIMICOLAE.

SCOLOPACIDAE.

N. H.- Rare migrant: Rye Beach, Aug. 27, 1868.

VT.- Formerly a migrant.

MLsss.- Rare migrant. May 20-23; Juy 17-Sept. 5.

R. I.- Rarre migrant. Aug. 6-Oct. 2.

Coxs.- Rare migrant. Aug.

150. Linosa haemastica (Limné) Cones.

Hudsonian godwit; Black-tail; Goose-bird; Ring-tailed marlin; Spot-rump.

Scolopax haemastica Limné, Syst. nat., ed. 10, 1758, vol. 1, p. 147. "in Anerica septextrionali."

Limosa hudsonica Lath. Audubon, Birds of Amer., 1842, vol. 5, p. 335 , pl. 349.

Beaches and marshes; nests on the ground.

Me.- Rare fall migrant coastwise.

N. H.- Rare fall migrant coastwise. Ang. 2-Oct.

VT.-Rare migrant: Burlington.

MAss.-Rare (? spring and) fall migrant. (" April 29-May 25); July 15-Nov. 7.

R. I. - Rare (? spring and) fall migrant coastwise. July 22.2Oct. 13.

Cosx-- Rare migrant.

151. 'Totaxes melaxoleucus (Gmelim) Vieillot.

Greater yellow-legs; Cucu; Greater tattler or telltale; Longlegged tattler; Stone snipe; Winter yellow-legs.

Scolopax melanoleuca Gmel., Syst. nat., 178s, vol. 1, pt. 2, p. (3.59.

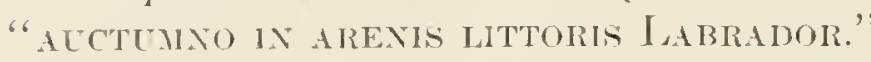

Totanus roriferus Wils. Audubon, Birds of Amer., 1St2, vol. 5, p. 316, pl. 34.5 .

Salt and fresh marshes; nests on the ground. 
LIMICOLAE.

\section{SCOLOPACIDAE.}

ME.-Common migrant and possibly a rare summer resident in extreme north (Woolastook valley). Late April-May 19 (July); late July-Oct. 22.

․ H.- Common migrant, manly cosstwise. May; July 17Oct. 27

VT.- Lneommon migrant. Maỹ fall.

Mass.-Common migrant maninly coastwise; a few non-breeding birds rarely summer. (Mar. 27) April t-June 16 (June 28); July 6-ヘог. 12.

R. I.-Common migrant. (? Malr. 10) April 10-May 20; July 20-Nor. 4.

Coxs-Common migrant. May 14-June 1 (June 13, 1S9s New London); Aug-Nov. 15.

152. Totaxus ruaripes (Gmelin) Ticillot.

\section{Yellow-legs; Lesser yellow-legs; Summer yellow-legs; Wan- dering tattler.}

Audubon, Birels of Amer., 1842, vol. 5, p. 313, pl. 34t. Ega, Seebohm, 1896, p. 139, pl. 44, fig. 11.

Scolopax flaripes Gmel., Sist. nat., 17Ss, rol. 1, pt. 2, p. 6.59. "in Noveboraco."

Salt and tresh marshes; nests on the ground.

ME.- Rare spring and common fall migrant. Maly 16 ; late July-(O)t: 17).

N. H. - Rare spring and common fall migrant. Maly; Aug.Sept. 4.

VT.- Uncommon migrant.

MAss. - Rate spring and common early fall migrant. April 29June 2 (June 15); July 10-Sept. 29) (O) (ot. 11 ind 30).

R. I.- Rase spring aud common fall migrant. April 2S; Iuly 3-O(.t. 1.

Coxx- Ralle spring and common fill migant. Maty 7 ; Aug. 17 Oc.t. 
LIMICOLAE.

\section{SCOLOPACIDAE.}

153. Helonromas solitarius (Wilson) Sharpe.

\section{Solitary sandpiper or tattler.}

Tringa solitaria Wils., Amer. ornith., 1813, vol. 7, p. 53, pl. 58, fig. 3. "Kentucky to New York."

Totanus solitarius (Wils.). Audubon, Birds of Amer., 1S42, rol. 5, p. 309, pl. 343 . Egg, Calpen, 1S56, pl. 19, fig. 6.

Borders of woodland streams and ponds, rarely near salt water; nests on the grom or in other birds' deserted nests.

Me.-Common migrant and in northern part a rare summer resident (Woolastook valley). May 7-30; summer-Oct. 2 (Oet. 21).

N. H.-Common migrant, and possibly a rare summer resident. May 9-June; July 17-(Nov. 1).

$V_{\mathrm{T}}$-C Common migrant; reported breeding at Lake Bomoseen, St. Albans Bay, Wells River. May-Sept. 3. Eggs, middle of May.

Mass.-Common migrant, mainly inland. May 2-26 (June); (July S) July 20-Oct. 14 (Nor. 2S).

R. I.- Uneommon migrant. May 3-20; July 18-Sept. 25.

Coxx.-Common migrant. May 2-(June 10); Aug.-Sept.

154. Catoptrophorus sempalmatus (Gmelin) Bonaparte.

\section{Willet; Goose-bird; Humility.}

Scolopax semipalmata Gmelin, Syrst. nat., 17SS, vol. 1, pt. 2, p. 659. "IN Noveboraco."

Totamus semipalmatus Lath. Audubon, Birds of Amer., 1St2, vol. 5, p. 324, pl. 347. Egg, Capen, 18S6, pl. 19, figs. 4, 5.

Salt and fresh marshes, and beaches; nests on the ground.

ME., N. H.- Rare migrant coastwise. Oet. 25.

VT.-Rare migrant: Middlebury.

Mass. - Rare migrant coastwise; formerly rare summer resident 
LIMICOLAE.

SCOLOPACIDAE.

(Muskeget, about 1869, New Bedford). Nay 2-31 (June 17); July S-Sept. 11.

R. I.- Rare migrant coastwise, mainly in fall. Nay; July 2 Sept. 18.

Cons. - Formerly a rare summer resident (Madison, Stratford), now only a rare migrant. May; (summer); Ang. 9-Oct. Eggs, June 5, 1873.

155. Catoptrophorus sempalahtus inornatus (Brewster) A. O. U. comm.

\section{Western willet.}

Symphemia semipalmata inornata Brewst., Auk, 1887, vol. 4, p. 145. "Lariner County, Colorado."

Totanus semipalmatus Temm. Swainson and Richardson, Fauna Boreali-Amer., 1S31, vol. 2, p. 38s, pl. 67.

Salt and fresh marshes, and beaches; nests on the ground.

Mass.- Rare fall migrant: Newburyport, Aug. 5, 1908 (and probably most of those now found).

Conv.- Rare fall migrant: Stony Creek, Aug. 15, 1897, and other fall birds.

156. Payoncella pugnax (Linné) Leach.

\section{$\operatorname{Ruff}\left(0^{\top}\right) ;$ Reeve (ㅇ).}

Tringa pugnax Linné, Syst. nat., ed. 10, 1758, vol. 1, p. 148. "IN EURopa Mines boreali."

Machetes pugnax Linné. Dresser, Birds of Europe, 1871-\$1, vol. S, p. [S7], p]s. [557, 55S]. Egg, Seebohm, 1896, p. 135, p]. 42, figs. 4,6 .

Shores and marshes; nests on the ground.

Me.-Accidental from Old World: Camden, Sept. 14, 1900; Searborough, April 10, 1870; Upton, Sept. S, 1874. 
LIMICOLAE.

SCOLOPACTDAE.

N. H.-Accidental visitor: Seabrook, Sept. 23, 1907.

Mass.-Accidental visitor: Chatham, Sept. 12, 1850; Nantucket, July, 1901; Newburyport marshes, Мay 20, 1 S71.

R. I.-Aecidental visitor: Point Judith, Aug. 31, 1903; Sakonnet, July 30, 1900.

157. Bartrami losgicald (Bechstein) Bonaparte.

Bartramian sandpiper; Field plover; Grass plover; Pasture plover; Upland plover.

Tringa longicanda Bechst., Küure uebers. vögel Lathams, 1S12, vol. 2, p. 45.3. North America.

Tringa bartramia Tils. Audubon, Birds of Amer., 1S12, rol. 5, p. 24S, pl. 327. Egg, Capen, 1SS6, pl. 19, figs. 9, 10.

Grassy prairies and fields; nests on the ground.

ME.- Common migrant, and uncommon local summer resident. April 19-summer; Aug. t-Sept. 16. Eggs. June 6.

\. H.- Now uncommon migrant and summer resident in southern part. May 4 -Sept.

VT.- Lncommon migrant and local summer resident. April 24Sept. Egrgs, May 26.

MAss.- Rare spring and uncommon fall migrant, rare local summer resident; formerly commoner. April 3-Nay 10; summer; July 16-Sept. 14. Eggs, May 25-June 3 (June 22).

R. I.- Uncommon migrant; formerly more common and probably bred. April; Aug. 2-Sept. 1S.

Coxs.- Formerly common summer resident; now an uncommon migrant. \Iay 2; fall.

15S. Tringites subruficollis (Ticillot) Ridgray.

Buff-breasted sandpiper; Hill grass-bird.

Tringa subruficollis Tieill., Nour. dict. d'hist. nat., 1S19, rol. 3t, p. 465. "PARAGLAY." 
Tringa rufescens Vieill. Audubon, Birls of Ancr., 1842, rol. 5, p. 264, pl. 331. Egg, Seebohm, 1S96, p. 151, pl. 43, fig. 11.

Grissy uplands and marshes; nests on the ground.

ME.- Rare fall miglant coastwise. Aug.-Sept. j.

N. H.- Rare fall migrant coastrise: Portsmouth; Rye Beach, Ang. 2.5 and 2S, 1871.

VT.-Doubtfully recorled.

MAss. - Rare fall migrant coastwise. July 2S-Sept. 24.

R. I.- Rare fall migrant coastwise. Sept. 6-15.

Cosx.- Rare fall migrant coastwise. Aug.-Sept. 30.

159. ACTitis MaCUlaria (Limné) Naumann.

Spotted sandpiper; Ox-eye; Peet-weet; Sand lark; Teeter peep; Tip-tail; Tip-up.

Tringa macnlaria Linné, Sịst. nat., ed. 12, 1766, rol. 1, p. 249. "in Europa \& Ainerica septextrionali."

Totanus macularius Wils. Audubon, Birds of Amer., 1S42, rol. 5, p. 303, pl. 342. Egg, Capen, 18\$6, pl. 19, figs. 7, S.

Seacoast and freshwater streams and lakes; nests on the ground.

ME.- Common migraut and summer resident. April 26Oct. 3 (Nor. 19). Eggs, May 20-July 1.

\. H.- Common migrant and summer resident at lower elerations. May 1-Oet. Eggs, May 31.

VT.-Common migrant and summer resident at lower elevations. Late April-Sept.

MAss.-Common migrant and summer resident. (April 19) April 26-Nor. 6 (Nor. 14). Egge, May 25-July 4.

R. I.-Common migrant and summer resident. (April 16)Oct. 3. Eggs, May 27-June 6.

Coxs.-Common migrant and summer resident. Late AprilOct. 6. Fgggs, May 31-June 1. 
LINIICOLAE.

$$
\text { SCOLOPACIDAE. }
$$

160. Numienius americanus Bechstein.

\section{Long-billed curlew; Great curlew; Hen curlew; Sickle-bill.}

Bechst., Kurze uebers. vögel Lathams, 1812, vol. 4, pt. 2, p. 432.

"New York \& Hudson Bay."

Numenius longirostris Wils. Audubon, Birds of Amer., 1843, vol. 6, p. 35, pl. 35.5 .

Marshes and grassy flats; nests on the ground.

Me.- Very rare migrant. May 2; Aug.

N. H.- Formerly rare, now accidental migrant. Aug. 12-25.

Mass. - Now an accidental migrant. July 20-Oct. 18.

R. I.- Now an accidental migrant; last record Jamestown, Sept. 9, 1897. July 15-Sept. 9.

Coxs.-Formerly uncommon, now accidental migrant. Aug. 3.

161. Numexius hudsonicus Latham.

Hudsonian curlew; Blue-legs (young); Foolish curlew (young); Jack curlew; Short-billed curlew.

Lath., Index ornith., 1790, vol. 2, p. 712. "IN sINu Hudsonis." Autulbon, Birds of Amer., 1S43, vol. 6, p. 42, pl. 356.

Beaches and marshes; nests on the ground.

Me.- Rare migrant. May 20; July 19-late Sept. (Oct. 12, 1898, Dover).

N. H.- Rare migrant. Aug. 6-Sept. 2.

MAss.- Rare spring and uncommon fall migrant. April 10May 30 (June S); (June 23) July 6-Oct. 1.

R. I.- Rare spring and uncommon fall migrant. April 27-May; July 2-Sept. 25.

Coxx.- Rare migrant, mainly in fall. July 20-Aug. 27.

162. Nunexies borealis (Forster) Latham.

Eskimo curlew; Dough-bird; Fute.

Swainson and Richardson, Fauna Boreali-Amer., 1831, rol. 2, p. 378 , pl. 65. Egg, Seebohm, 1896, p. 133, pl. 45, fig. 3. 
LIMICOLAE.

\section{SCOLOPACIDAE.}

Scolopax borealis Forst., Phil. trans. roy. soe. I.ondon, 1772, rol. 62, pp. 411, 431. "Almany For'T," Hudoson BaY.

Marshes, beaches, and grassy uplands; nests on the ground.

Me., X. H.- Folmerly common fall migrant, coastwise. Iate Aug.-Sept.

MAss. - Formerly a common fall migrant, accidental in spring. (Late May 1873, Cape Cod); July 15-Oct. 2.

R. I.- Folmerly a common fall migrant coastwise. Aug. 27Sept.

Coxx- Formerly a common fall migrant coastwise, now very rare. Aug.-Oet. 13.

\section{CHARADRIIDAE.}

163. Squatarola squatarola (Limné) Cuvier.

Black-bellied plover; Beetlehead; Blackbreast; Blackheart; Frost-bird; Ox-eye; Palebelly (young); Whistling field plover.

Tringa squatarola Limmé, Sỵst. nat., ed. 10, 1758 , rol. 1, p. 149. "IN Europa."

Charadrins helveticus Linné. Audubon, Birds of Aner., 1842, rol. 5, p. 199, pl. 315.

Beaches and marshes; nests on the ground.

Me., N. H.- Common migrant chiefly coastwise. May 12(June 24); Aug.-Oct. 24.

YT.- Rare migrant: Lunenburg; Middlebury.

Mass.- Common migrant coastwise. April 15-June 13 (June 25); July S-Nov. 16 (Dec.).

R. I.-Common migrant. Nay 15-22; Aug. 9-Oct. 15.

Coxx.- Common migrant coastwise. 
LIMICOLAE.

\author{
CHARADRIIDAE.
}

164. Charadrics dominicis Müller.

American golden plover; Bullhead; Frost-bird; Greenback; Greenhead; Green plover; Muddy breast; Pale belly; Pale breast; Three toes; Toad-head.

IIüll., Limné's Vollstand. naturșrst., suppl., 1776, p. 116. "ST. Domago."

Charadrius marmoratus Wagl. Audubon, Birds of Amer., 1842 . vol. 5, p. 203, pl. 316. Egg, Seebohm, 1S96, p. 125, pl. 39, fig. 5.

Beaches, marshes, and open hilltops; nests on the ground.

ML.-Formerly common, now rare fall migrant coastrise. Aug. 12-Nor. 15.

N. H.- Rare migrant coastwise. (Mar); Aug. 26-mid-Oct.

TT.- Rare migrant: Lunenburg.

Mass.- Tery rare spring, and formerly common but now rare fall migrant coastrise. (May); Aug. 12-Nor. 2 (Nor. 19; Nor. 25, 190s, Ipswich).

R. I.- Migrant, formerly rare in spring and common in fall coastwise; now very rare. (May); Aug. 14-Oct. 25.

Coxx-Formerly common migrant, now rare; mainly coastwise. Oct. 24.

165. Oxvechl's vocireres (Linné) Reichenbach.

\title{
Killdeer.
}

Charadrius vociferus Jinné, Sỵst. nat., ed. 10, 17js, vol. 1, p. 150. "M Alierica septextrioxali."

Aegialitis rocifera (Limné). Chapman, Handb. birds east. No. Amer., 1595, p. 173; Bird-life, 189S, pl. 11. Egg, Capen, 1886, pl. 18, figs. \&, 9.

Meadors, fields, and marshes; less often on beaches; nests on the ground.

ME.- Rare migrant and accidental risitor. Aug. 1i-Oct. 24 (Nor. 29-Dee. 4, 1888). 
LIMIICOLAE.

$$
\text { ('IIARATRIII)AE. }
$$

N. H.- Rane migrant and aredidental winter resident (1SSS-S9); formerly rare summer resident.

V'T.- Rare migrant and summer resident (Lake Clamplain). MAss-C Rare migrant and summer resident; accidental winter resident (1SSS-S9). Mar. 1-Der. 15 (winter). Jyggs, May 30.

R. I.- Lncommon migrant and rare smmmer resident; accidental winter resident in 1SSS-S9. Mar.-Dec. (winter). Eggrs, May 10-31.

Cons.- Rare migrant and summer resident, formerly more plentiful. (Feb. 24) late Mar,-Mily; summer. Eggs, June 6.

166. Akglalitis semipaliata (Bonaparte) Bomaparte.

Semipalmated plover; Little ring-neck; Ring-neck.

Charadrins semipalmatus Bonap., Jomm. acad. nat. sci. Phila., 1S25, rol.5, p.9\$. Bascd on Wilson: "NEW Jerser." Audubon, Birds of Iner., 1St2, rol. .j, p. 21s, pl. 320.

Siludy beaches and salt-marsh flats; nests on the ground.

ME.-Common migrant; mainly coastwise. May-(June 22); July 29-Sept.

‥ H.- Common migrant, mainly coastwise.

l'T.- Rare migrant.

MAss.-Common migrant, mainly coastwise. Late April-June $1+$ (.June 26); July 12 - O). 28.

R. 1.-Common migrant, manly coastwise. April 19-Мау 15; July 6-Sept. 25.

Coxx- Common migrant, mainly coastwise. May 17-June; July-Sept.

167. Afgialtis Malod (Ord) Bonaparte.

Piping plover; Clam-bird; Mourning-bird; Pale ring-neck; Ring-neck.

Charadrius melodus Ord, Wilson's Amel. omith., 1524, vol. 7, 
LIMICOLAE.

\section{CHARADRIIDAE.}

p. 71. "Great Egg-Harbor," N. J. Audubon, Birds of Amer., 1842, vol. 5, p. 223, pl. 321. Egg, Capen, 1SS6, pl. 1S, fig. 10.

Sandy beaches; nests on the ground.

Me.- Formerly a rare summer resident coastwise (IVells Beaeh). May 2-Aug.

N. H.- Rare migrant coastwise.

VT.-Doubtfully lecorded.

MAss.- Uncommon migrant and summer resident coastwise. (Mar. 26) April 5-Sept. 22. Eggs, May 10-June 14 (July 20).

R. I.- Unconmon migrant and summer resident coastwise. (Mar. 24) May 15-Sept. Eggs, Jume 5-14.

Cons.- Uncommon migrant and summer resident coastwise.

168. Ochthodronus wilsonius (Ord) Reichembach.

\section{Wilson's plover.}

Charadrius wilsonia Ord, Wilson's Amer. ormith., 1S14, vol. 9, p. 77, pl. 73, fig. 5. "Cape Island, Newjersey." Audubon, Birds of Amer., 1842, vol. 5, p. 214, pl. 319.

Sandy beaches and mudflats; nests on the ground.

VT., N. H.- Doubtfully recorded.

Mass.-Accidental visitor: Gernet Pt., Plymouth, Aug. 22, 1877; Ipswich, May S, 1904 (nec Nahant, Aug. 1839).

R. I.- Doubtfully included.

Conv.-Accidental visitor": Bridgeport, July 2S, 1858 ("scen," Averill); Stratford (Linsley).

\section{APHRIZIDAE.}

169. Arenaria interpres (Limné) Vieillot.

\section{European turnstone; Tangle-picker.}

Tringa interpres Linné, Syst. mat., ed. 10, 1758, vol. 1, p. 148. "In Europa \& Ailerica septentrionali." 
LIMICOLAE.

APHRIZIDAE.

Strepsilas interpres (Linné). Dresser, Birds of Europe, 1S71-S1, vol. 7, p. [55.5], pl. [532].

Seacoasts; nests on the ground.

MAss.-Accidental visitor: Monomoy Island, Sept. S, 1S92, (Bishop).

170. Arenaria interpres morinellat (Linné) A. O. U. comm.

Ruddy turnstone; Bead-bird; Brant-bird; Calico-back; Calicobird; Chicken plover; Horsefoot snipe; Rock-bird; Rock plover; Stone plover; Turnstone.

Tringa morinella Linné, Sỵst. nat, ed. 12, 1766, rol. 1, p. 249. "AD MARIS LITTORA AMERICAE SEPTENTRIONALIS; \& EUROPAE."

Strepsilas interpres (Linné). Audubon, Birds of Amer., 1S42, vol. 5, p. 231, pl. 323 .

Seacoasts; nests on the ground.

Mr.-Common migrant. May 22-27; July 2S-Sept.

N. H.- Uncommon migrant.

VT.- Rare migrant: Lake Champlain.

Mass.-Uncommon migrant. May 1-31 (June 2t); July 25-Oct. 16.

R. I.- Common migrant. May 14-20; Aug. 4-Oct. S.

Cons.- Common migrant. May 23; Aug.-Sept.

\section{HAENATOPODIDAE.}

171. Haenatopes Pallates 'lemminck.

\section{American oyster-catcher.}

Temm., Miln. d'ornith., ed. 2, 1S20, vol. 2, p. 532. "L'AMÉrIQUE MÉridionale." Audubon, Birds of Amer., 1St2, rol. 5, p. 236, pl. 324. Egg, Gentry, Nests and eggs birds U. S., 1SS2, p. $277, \mathrm{pl} .47$.

Seacoasts; nests on the ground. 
LIIICOLAE.

HAEMATOPODIDAE.

Me.- Formerly at Portland (Audubon).

Mass - Now an accidental visitor. Late April; Aug.

Coxs.- Now very rare migrant; formerly more plentiful.

GALLINAE.

PHASIANI.

\section{TETRAONIDAE.}

172. Colines rirginiaxes (Limné) Stejneger.

Bob-white; Quail; Virginia partridge.

Chapman, Handb. birds east. No. Amer., 189.5, p. 17.9, pl. 1. Egg. Bendire, 1892, vol. 1, pl. 1, fig. 1.

Tetrao virginiamus Linné, Srst. nat., ed. 10, 175s, vol. 1, 1. 161. "Anierica."

Weedy and brushgrown fields and edges of woods; nests on the ground.

Me., N. H. - Formerly common, now rare resident in southern counties; also introduced from the South.

TT.-Rare resident at lower elevations; largely introduced.

MAss-Uncommon resident; also introduced. Eggs, MayJune 30 (Sept.).

R. I.-Common resident; also frequently introduced. Eggs, May 2.5-June 20 (! Oct. 10).

Coxx-Common resident, also introduced. Eggs, June 14Aug. (! Sept. 2).

173. Canarhites Caxidexsts Cavace (Limné) Norton.

Canadian spruce grouse; Black grouse; Fool-hen; Spotted grouse; Spruce partridge; Wood partridge.

Tetrao conace Linné, Syrst. nat., ed. 12, 1766, rol. 1, p. 275. "CA.idDa."

Tetrao ranadensis Linné. Audubon, Birds of Amer., 1\$42, vol. 5, p. \$3, pl. 294. Egg, Bendire, 1892, vol. 1, pl. 1, fig. 23. 
GALIAIAE.

TETRAONII)AE.

Dense fir and spruce woods; nests on the ground.

Me.- Lncommon resident in northern forests. Eggg, May.

N. H.- Lncommon resident in northern part, and abore 3000 ft. in White Mts. south to Mt. Passaconaway. Eggs, late May.

VT.- Uneommon resident in northermmost part (Victoria).

Mass - Aceidental visitor: Gloncester, Sept. 1851; Roxluury Nor. about 1565 .

17t. Boxasa umbeluts (Limné) Stephens.

Ruffed grouse; Birch partridge; Partridge; Pheasant.

T'etrao umbellus Linné, Syst. nat., ed. 12, 1766, rol. 1, p. 275. "ix Pexsilianta." Audubon, Birds of Amer., 1st2, vol. 5, p. 33 , pl. 293. Egg, Bendire, 1892, vol. 1, pl. 2, fig. 1.

Woods and thickets; nests on the ground.

VT.- Nearly typieal birds rarely found in south part; resident. Mass- Uncommon resident. Eggs, April 27-June?.

R. I.-Common in northern and western parts. Eggs, Mayy S19.

Coxx-Common resident. Eggs, April 21-May 20.

175. Bonasa cmbelles togata (Linné) Ridgway.

\section{Canadian ruffed grouse; Partridge; Pheasant.}

Riulgway, Mammal No. Amer. birds, 1ss7, p. 19\$. Egg, Bendire, 1892 , vol. 1, pl. 2, fig. ‥

Tetrao togatus Limé, Sỵst. nat., ed. 12, 1766, vol. 1, p. 275. "CANADA."

Woods and thickets; nests on the ground.

Me.- Common resident. Eggs, April 27-Mary 25.

N. H.-Common resident up to tree limit on monntains.

IT.-Common resident. 
GALLINAE.

PHASIANI.

TETRAONIDAE.

Mass.- Resident; nearly typical birds occur in Berkshire Co. (North Adams).

176. Lagorus lagopus (Linné) Stejneger.

\section{Willow ptarmigan.}

Tetrao lagopus Linné, Syst. nat., ed. 10, 1758, vol. 1, p. 159. "in Europae alpinis."

Lagopus albus Gmel. Audubon, Birds of Amer., 1S42, vol. 5, p. 114, pl. 299 (summer). Egg, Bendire, 1892, vol. 1, pl. 2, fig. 510.

Dense thickets; nests on the ground.

Me.-Accidental visitor: near Dennisville (Audubon); Kenduskeag, April 23, 1892.

MAss.-Accidental visitor (or escape): Manchester, May 10, 1859 .

R. I.- Doubtfully recorded.

\section{Tympanuchus cupido (Linné) Ridgway.}

\section{Heath-hen; Eastern pinnated grouse; Hethen.}

Tetrao cupido Linné, Srvst. nat., ed. 10, 1758, vol. 1, p. 160. “IN Virginia." De Kay, Zool. New York, 1S44, vol. 2, p. 205, pl. 77 , fig. 175.

Cupidonia cupido Brewster, Auk, 1885, rol. 2, p. S2. Egg, Bendire, 1892, vol. 1, pl. 3, fig. 2.

Scrub-oak thickets; nests on the ground.

Me.-Ascribed by Audubon to Maine, but perhaps erroneously. Mass.- Resident, now confined to Martha's Vineyard, but (till about 1815) oceurring coastwise nearly to Boston, and in lower Connecticut ralley. Eggs, Jume 2-July 24 .

R. I.- Formerly common, but practically extirpated by 1800 .

Cors.- Formerly common; extirpated before 1840 (Westford, about 1832 , Nuttall). 
GALLINAE.

TETRAONIDAE.

17S. Meleagits Gallopavo silvestris (Vicillot) Allen.

\section{Wild turkey.}

Meleagris silvestris Vieill., Nour. diet. d'hist. nat., 1817, vol. 9,

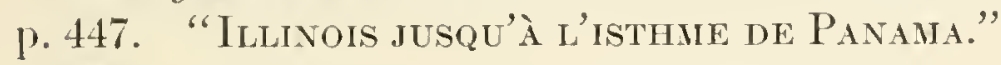

Melcagris gallopavo Linné. Audubon, Birds of Amer., 1842, vol. 5, p. 42, pls. 287, 2SS. Egg, Bendire, 1S92, vol. 1, pl. 3, fig. 14.

Woods and thickets; nests on the ground.

ME.-Formerly a resident in southern counties (to Mt. Desert?); now extirpated.

N. H.- Formerly a resident in southern part; last record in 1841 or' 42.

VT.- Formerly resident in southern part; now extirpated.

MAss.- Formerly common resident; last recorded eapture at Mt. Tom, winter of $1850-51$.

R. I.- Formerly common resident.

Conn. - Formerly common resident; last killed about 1813 on Totoket MIt., Northford.

COLUMBAE.

\section{COLUMBIDAE.}

179. Ectopistes migratorius (Linné) Swainson.

\section{Passenger pigeon; Wild pigeon.}

Columba migratoria Linné, Syst. nat., ed. 12, 1766, vol. 1, p. 255. "IN Anerica septentrionali."

Ectopistes migratoria (Limné). Audubon, Birds of Amer., 1842, vol. 5, p. 25, pl. 285. Egg, Bendire, 1892, vol. 1, pl. 4, fig. 6.

Woods and fields; nests in trees in colonies.

MF.- Formerly an abundant migrant and local summer resident; last specimens taken near Dexter, Aug. 16, 1896, and at Bar Harbor, early summer 190t. April 7-Sept. 
COLLIBBAE.

\section{COLLMBIDAE.}

N. H.- Formerly abundant migrant and local summer resident; last specimen taken at Concord, 1SS5. April 2-18; summerOct. 10.

VT.- Formerly abundant migrant and summer resident. Mar. 20-Oct. 10.

MAss.- Formerly abundant migrant and common local summer resident, rare in winter; last recorded in 1859. MIar. 10-Oct. 21 (winter). Eggs, May 22.

R. I.- Formerly abundant migrant; last killed in 1 SS6.

Coxs.- Formerly an abundant migrant, and common summer resident. April 2-fall. Eggs, late May.

1S0. Zexaidura aiacioura Carolinexsis (I immé) A. O. L'. comm.

Mourning dove; Carolina dove; Long-tailed dove; Turtle dove.

Columba carolinensis Linné, Șrst. nat., ed. 12, 1766, rol. 1, p. 286. " "IN AvieriCA."

Zenaidura macroura (I imné). Chapman, Handb. birds east. No. Amer., 1895, p. 18s; Bird-life, 189s, pl. 13. Egg, Bendire, 1892, vol. 1, pl. 4 , figs. $8,9$.

Open woods and fields; nests in trees, or rarely on the ground.

ME.- Rare migrant and summer resident in southern part; accidental in winter. Mar. 21-Oct. 24 (Nor. 21 and winter).

N. H. - Rare migrant and summer resident in southern part. Late Mar.-Oct. (Dec. 15).

TT.- Rare summer resident: Rutland Co.

MAss.- Uncommon migrant and summer resident; rare winter resident. Mar. j-Nov. 15 (minter). Fggs, April 19-May 20.

R. I.- Uncommon summer resident in northern and western parts. April 3-Oct. 15. Eggs, May 15-25.

Coxx.- Uncommon summer and rare winter resident. Mar.Nov. (winter). Eggs, May 24-June 20 (July 27). 
COLUMBAE.

$$
\text { COLLABIDAE. }
$$

1S1. Stariognas cranociphata (limué) Bomaparte.

\section{Blue-headed quail-dove.}

Columba cyanocephala Linné, Syst. nat., eal. 10, 17:5, rol. 1, p. 163. "Aluericı." Audubon, Birds of Amer., 18t2, vol. 5, 1). 23, p]. $25 t$.

'Thickets and woods; nests among vines.

MAss.- One taken at Brookline, Oct. 10, 1570, probably an escape.

RAPTORES.

SARCORHAMPHI.

$$
\text { CATHARTIJAE. }
$$

1S2. Cathartes atra septentrionals (Wied) Nelson.

\section{Turkey vulture; Turkey buzzard.}

Cathartes septentrionalis IVied, Reise Nord-America, 1S39, rol. 1, p. 162. Locality elsewhere given as NeAR NEw HARMONY, INDIANA.

Cathartes aura Limné. Audubon, Birds of Aner., 1St0, vol. 1, p. 15, pl. 2. Egg, Bendire, 1s92, vol. 1, pl. 4, figs. 1, 3.

Open country; nests on ground in shallow caves or in hollow logs.

ME.-Accidental visitor at all seasons (eight records).

X. H.- Accidental visitor: Hampton Falls, April 1SS2; North Weare, spring 1 ss7.

VT.-Accidental risitor: Eutaw.

Mass-Oceasional visitor at all seasons.

R. I.- Occasional visitor; four records. May 10-Nor.

Coxx.-Occasional (mainly summer) visitor. April 20 -Oct. 1 S.

1S3. Catharista crube (Vicillot) Vicillot.

Black vulture; Black-headed buzzard; Carrion crow; Jim crow.

Fultur urulu Vieill., Hist. nat. ois. Amér. Sept., 1S07, vol. 1, 
RAPTORES.

SARCORHAMPHI.

CATHARTIDAE.

p. 23, pl. 2. “L’Anérique, depuis la Nouvelle-Ecosse jusqu'À la T'Terre de Feu."

Cathartes atratus Wils. Audubon, Birds of Amer., 1840, vol. 1, p. 17, pl. 3. Egg, Bendire, 1892, vol. 1, pl. 4, figs. 7, 10 .

Open country and vicinity of dwellings; nests on the ground. Me.-Accidental risitor. Aug. 20-Nov. 3.

VT.-Accidental visitor: Lunenburg; Woodbury, July 10, 1884.

Mass.- Accidental risitor. July-Nor.

Conv.-Accidental visitor: East Lyme, July 6, 1901.

\section{FALCONIDAE.}

FALCONES.

184. Elanoides forficatus (Limé) Coues.

\section{Swallow-tailed kite.}

Chapman, Handb. birds east. No. Amer., 1895, p. 196; A. K. Fisher, Hawks and owls of U. S., 1S93, pl. 1. Egg, Bendire, 1892, vol. 1 , pl. 5, figs. $1,2$.

Falco forficatus Linné, Syst. nat., ed. 10, 1758, vol. 1, p. $\$ 9$. "Anerica."

Open country; nests in tall trees.

ME.- Erroneously recorded.

N. H.- Doubtfully recorded (Franklin, 1875).

VT.-Doubtfully recorded (Williams).

Mass.-Accidental visitor: Amesbury (= West Newbury) about Sept. 25, 18S2; Whately; (?) near Northampton, 1880.

Conv.-Accidental visitor: Lyme, July 2, 1877; Portland, summer 1S61; Saybrook, June 16, $18 S 9$.

185. Circus hudsonius (Linné) Vieillot.

Marsh hawk; Blue hawk; Bog-trotter; Frog hawk; Harrier; Mole hawk; Mouse hawk; Mouser; Snake hawk.

A. K. Fisher, Hawls and owls of U. S., 1893, p. 26, pl. 3. Egg, Bendire, 1892, vol. 1, pl. 5, fig. S-10. 
RAPTORES.

FALCONES.

FALCONIDAE.

Falco hudsonius Iinné, Syst. nat., ed. 12, 1766, vol. 1, p. 128. "AD Fretun Hudsonis."

Open country, fields, marshes; nests on the ground.

Me.- Common migrant, less common summer resident. Mar. 19-Oet. 7 (Nov. 12). Eggs, June 4.

N. II.- Common migrant, uneommon summer resident. Mar. 16-Nor. 12.

VT.- Uncommon summer resident. April 14-Oet.

Mass.- Common migrant, uncommon summer resident; rare winter resident on the coast. Mar. 1-Nov. 29 (winter). Eggrs, May 12-June $S$.

R. I.-Common migrant, rare summer and winter resident. Mar. 15-Oct. 29 (winter). Eggs, May 23, 25.

Cons.- Common migrant, uneommon summer resident; winters rarely. Mar.-Nov. 6 (winter). Eggs, May 9-June 9.

1S6. Acciplter velox (Wilson) Vigors.

\section{Sharp-shinned hawk; Pigeon hawk.}

Chapman, Handb. birds east. No. Amer., 1595, p. 19S; A. K. Fisher, Hawks and owls of U. S., 1S93, pl. 4. Egg, Bendire, 1892, vol. 1, pl. 5, fig. 11-17.

Falco velox Wils., Amer. ornith., 1812, vol. 5, p. 116, pl. 45, fig. 1. "BANKS OF THE SCHUYLKiLl."

Open or wooded country; nests in trees.

ME.-Common migrant, less common summer resident; rare winter resident (Portland, 1905; Westbrook, 1907). Mar. 27Nor. 23 (winter). Eiggs, June 1.

N. H.- Common migrant, less common summer resident; rare winter resident in southern part. Egrgs, May 23-June 1.

$V_{\mathrm{T}}$.- Uncommon summer and rare winter resident.

MAss.-Common migrant, uncommon summer and winter resident. Eggs, May 3-June 10. 
RAPTORES.

FALCONES.

\section{FALCONIDAE.}

R. I.- Uncommon migrant, rare summer and winter resident. Eggs, May 23, 25.

Coxs.-Common nigrant, less common summer and winter resident. Eggs, May 15-June 26.

1S7. ACCipiter cooperil (Bonaparte) Gray.

\section{Cooper's hawk; Chicken hawk.}

Chapman, Handb. birds east. No. Amer., 1895, p. 199; A. K. Fisher, Hawks and owls of U. S., 1893, pl. 5. Egg, Bendire, 1892, vol. 1, pl. 5, fig. 1S-20.

Falco coopcrii Bonap., Amer. oruith., 1S2S, vol. 2, p. 1, pl. 10, fig. 1. "Near Bordentown, New Jersey."

Open country and woods; nests in trees.

Me.-Uncommon summer resident. April 4-Oct. 24 (? Jan. $16)$.

N. H.- Lncommon migrant and summer resident at lower elevations. Mar. 26-Oct. Eggs, May 12-30.

${ }_{\mathrm{T}}$.- - Uncommon migrant and summer resident; (? winter). Eggs, April 22.

Mass. - Uncommon migrant and summer resident; rare in winter. Eggs, April 25-June 11.

R. I.- Common migrant, less common summer resident; probably winters rarely. Eggs, May 12-14.

Conn.-Common migrant, less common summer and winter resident. Eggs, April 25-June 18.

1SS. Astur atricapillus (Wilson) Bonaparte.

\section{American goshawk; Blue hawk; Partridge hawk.}

Falco atricapillus Wils., Amer. ornith., 1S12, vol. 6, p. S0, pl. 52, fig. 3. Near "Philadelphia."

Accipitcr atricapillus (Wils.). Chapman, Handb. birds east. 
No. Amer., 1595, p. 199; A. K. Fisher, Hawks and owls of U. S., 1\$93, pl. 6. Egg, Bendire, 1\$92, vol. 1, pl. 6, fig. 1.

Open country and heary woods; nests in trees.

Me.-Common winter resident, less common summer resident. Oct. 26-May; summer. Eggs, April 25-May.

N. H.- Irregularly common winter resident; rare summer resident south to Alstead.

VT.- Irregular winter visitor; rare summer resident south to Rutland, Mendon. Eggs, April 23-May 1.

Mass. - Winter visitor, irregularly common; casual summer visitor (Hoosac Mt., Aug. 15, 1900). Oct. 4-Feb. 26.

R. I.- Irregular winter visitor, sometimes common. Oct. 27Feb. 14.

Coxs.- Irregular winter visitor, sometimes common; accidental in late summer. (Aug. 24, 1S93, West Haven) Oct. 2-winter.

189. Buteo borealis (Gmelin) Vieillot.

\section{Red-tailed hawk or buzzard; Hen hawk.}

Chapman, Handb. birds east. No. Amer., 1895, p. 200; A. K. Fisher, Hawks and owls of U. S., 1893, pl. 7. Egg, Bendire, 1892, vol. 1 , pl. 6 , figs. 5,6 .

Falco borealis Gmel., Syst. nat., 1785, vol. 1, pt. 1, p. 266. "IN America boreali, potissimuli Carolina."

Open country and forests; nests in large trees.

ME.- Lncommon summer resident, and in southern counties rare winter resident. Eggs, May 6-26.

N. H.- Uncommon resident, wintering from White Mts. south.

VT.-Uncommon resident. Eggrs, April 22.

MAss.- Lncommon migrant and winter resident; rare summer resident. Egggs, April 6-27.

R. I.- Uncommon winter, and rare summer resident. Nor. 30Mar. 25; summer. Eiggs, April j-30. 
RAPTORES.

FALCONES.

FALCONIDAE.

Coxx.- Lncommon migrant and winter resident; rare summer resident. Sept.-Mar.; summer. Eggss, Mar. 28-April 24 (Mar 25).

190. Buteo lineatus (Gmelin) Vieillot.

Red-shouldered hawk; Chicken hawk; Hen hawk; Winter hawk.

Chapman, Handb. birds cast. No. Amer., 1595, p. 201; A. K. Fisher, Hawks and owls of U. S., 1893, pl. S. Egg, Bendire, 1892, rol. 1 , pl. 7 , fig. $1-5$.

Falco lineatus Gmel., Syst. nat., 178S, vol. 1, pt. 1, p. 268. "IN instla Longa," New York.

Open country and woods; nests in large trees.

Me.-Uncommon summer resident. (Mar. 4) Mar. 20-Oct. 21. Eggs, late April-May 24.

N. H.- Uncommon summer resident north to southern valleys of White Mts. Mar. 27-Nor.

VT.-Uncommon summer resident (? winters). Mar. 17-fall. Eggs, April 22.

MAss.-Common migrant, less common summer and winter resident. Eggs, April 3-June 6.

R. I.-Uncommon resident. Eggs, April 5-13.

Coxs.-Common migrant and winter resident, less common summer resident. Eggs, April 5-June 1.

191. Buteo swainsoni Bonaparte.

\section{Swainson's hawk.}

Bonap., Geogr. and comparative list birds Europe and No. Amer., 1838, p. 3. Based on Audubon: "xear the Colcubia River." Chapman, Handb. birds east. No. Amer., 1895, p. 203; A. K. Fisher, Hawks and owls of L. S., 1593, pl. 9. Egg, Bendire, 1892, vol. 1, pl. S, fig. 1-6. 
RAPTORES.

FALCONES.

FALCONIDAE.

Open prairie country or edges of woods near streams; nests in trees.

Me.-Occasional visitor from West. May 19; Sept. 15, Oct. S. T.- Rare visitor: Lunenburg.

MAss. - Rare visitor (four records). April 20-Nov. 7.

192. Buteo platypterus (Tieillot) Faxon.

\section{Broad-winged hawk.}

Sparvius platypterus Yieill., Encycl. méthod. ornith., 1823, vol. 3, p. 1273. Based on Wilson: "NeAR the SchuYlkild."

Buteo latissimus (Wils.). Chapman, Handb. birds east. No. Amer., 1595, p. 204; A. K. Fisher, Hawks and owls of L. S., 1893," pl. 10. Egg, Bendire, 1S92, vol. 1, pl. 7, fig. 10-13.

Woodlands, and along streams; nests in trees.

ME.- Uncommon summer resident. (Mar. 31) April 11-Sept. 17. Eggs, May 2-June 12.

X. H.- Lncommon migrant and summer resident. April \&Sept. 15. Eggs, May 16.

VT.-Uncommon summer resident. April 14-fall.

MAss. - Uncommon migrant, and in remoter districts, a rare summer resident. April 12-Sept. 30 (Oct. 16). Eggs, May 7-31.

R. I.- Uncommon migrant and rare summer resident. AprilOct. Eggs, May 15-19.

Coxs- - Uncommon migrant, and rare summer (and ? winter) resident. Eggs, Mİay 3-18.

193. Archibuteo lagoples saxcti-Johaxwis (Gmelin) Cones.

\section{American rough-legged hawk; Black hawk.}

Chapman, Handb. birds east. No. Amer., 1895, p. 205; A. K. Fisher, Hawks and owls of U. S. 1893, pl. 11. Egg, Bendire, 1892, vol. 1, pl. S, fig. 10-12. 
RAPTORES.

FALCONES.

FALCONIDAE.

Falco S. johannis Gmel., Sỵst. nat., 17S8, rol. 1, pt. 1, p. 273. "IN FRETO HudSONIS ET NOVA TERRA."

Open country; nests on elifts or in trees.

Me.- Uncommon fall and winter risitor. Oct. 28-Mar.

N. H.- Uncommon irregular fall and winter visitor. Oct.$(\operatorname{May} 6)$.

VT.- Rare fall and winter visitor. Oct.

MAss.- Rare fall and winter visitor. Oet.-April 13 (May S).

R. I.- Uncommon fall and winter visitor. Oet.-April 12.

Coxs.- Fall and winter visitor, irregular, but sometimes common. Nor. 20-April.

194. Aquila CHRYsä̈tos (Linné) Sprüngli.

\section{Golden eagle; Ring-tailed eagle.}

Chapman, Handb. birds east. No. Amer., 1595, p. 206; A. K. Fisher, Hawks and owls of U. S., 1893, pl. 13. Egg, Bendire, is92, vol. 1, pl. 9, figs. 3,5 .

Falco chrysaëtos Linné, Syst. nat., ed. 10, 1758, vol. 1, p. 88. “IN EURopa."

Mountainous country; nests on eliffs or in large trees.

MIE.- Rare visitor; not now known to breed (roung (?), Sandy Bay Mit., 1895).

N. H.- Rare visitor; formerly rare resident in White MIts.

VT.-Rare visitor; formerly a resident.

Mass.- Rare risitor at all seasons, not now known to breed.

R. I.- Rare visitor in fall and winter.

Coxx.- Rare visitor, at all seasons.

195. Halaeetus letcocephalus (Limné) Boie.

Bald eagle; Gray eagle; Washington eagle (young); Whiteheaded eagle.

Chapman, Handb. birds east. No. Amer., 1895, p. 207; A. K. 


\section{FALCONIDAE.}

Fisher, Hawks and owls of U. S., 1893, pl. 14. Egg, Bendire, 1S92, rol. 1, pl. 9, fig. 7 .

Falco leucocephalus Linné, Syst. nat., ed. 12, 1766, rol. 1, p. 124. "In Ainerica, Europa."

Open wild country, especially near lakes and seacoasts; nests in tall trees or on the ground.

ME.- Ǔncommon migrant and summer resident; less common minter resident coastwise. Eggs, April 4-21.

\. H.- Uncommon migrant and summer resident; rare in winter in southern part.

VT.-Uncommon migrant and summer resident.

MAss.- Uncommon migrant and, near coast, occasional at all seasons. No recent breeding records (formerly on Mt. Tom).

R. I.- Oceasional at all seasons, though not known to breed.

Coxx.- Occasional at all seasons, but most frequent as a migrant; formerly bred (MIt. Carmel, Saltonstall Ridge, West Peak):

196. Falco islandus Brünnich.

\section{White gyrfalcon; White or Speckled hawk.}

Brïnn., Ornith. borealis, 1764, p. 2. "Islandi."

Falco islandicus Lath. Audubon, Birds of Amer., 18t0, vol. 1, p. S1, pl. 19. Egg, Seebohm, 1S96, p. 4, pl. 1, fig. 2.

Open country and barrens; nests on sea cliffs.

ME.-Aceidental visitor: South Winn, Oet. S, 1S93; (nec Cape Elizabeth).

N. H.- Doubtfully recorded (Errol, Nov. 4, 1S6S, Maynard).

Mass.- Doubtfully recorded (Cambridge, Jan.-Feb., 1S33, Nuttall).

197. Falco Rusticoluts Linné.

\section{Gray gyrialcon.}

Linné, Syrst. nat., ed. 10, 1758, vol. 1, p. SS. "IN Svecia." 
RAPTORES.

FALCONES.

\section{FALCONIDAE.}

Coues, Key to No. Amer. birds, ed. 5, 1903, vol. 2, p. 665; Brisson, Ornith., 1760, vol. 1, pl. 31.

Open country; nests on cliffs.

ME.-Accidental visitor: Cape Elizabeth, Oct. 13, 1877.

19S. Falco Rusticolus grrfalco (Linné) Stejneger.

\section{Gyrfalcon; MacFarlane's gyrfalcon.}

Ridgway, Manual No. Amer. birds, 18s7, p. 245-2 46.

Falco gyrfalco Linné, Syst. nat., ed. 10, 1758, vol. 1, p. 91. "IN Europג." Dresser, Birds of Europe, 1871-\$1, vol. 6, pl. [367]. Egg, Bendire, 1S92, vol. 1, pl. 9, figs. 6, S, 9 .

Open or mountainous country; nests on eliffs.

ME.- Very rare winter visitor: Jacques Id., Mar. 7, 1907 ; Ktaadn Iron Works, December, 1S76; "MIaine;" North Deering, Dec. 11, 1906.

Tr.-Doubtfully recorded.

Mass.- Very rare winter visitor: Melrose, Jan. 1, 1891; Northanpton, Feb., 18S0; Stowe, $18 \$ 1$.

R. I.- Very rare winter visitor: Point Judith, Oct. 11, 18s3; near Providence, winter, 1864-65.

199. Falco rusticolus obsoletus (Gmelin) Stejneger.

\section{Black gyrfalcon; Labrador gyrfalcon.}

Ridgway, Manual No. Amer. birds, 1857, p. 245-246.

Falco obsoletus Gmel., Ș̣st. nat., 178S, vol. 1, pt. 1, p. 26S. "1N FRETO HuDSONis."

Falco labradora Audubon, Omith. biogr., vol. 2, pl. 196. Figg, Bendire, 1892, vol. 1, pl. 10, fig. 1.

Open and mountainous country; nests on cliffs or rarely in trees.

ME.- Rare fall and winter visitor. Sept.-Mar. 22.

N. H.- Rare winter visitor: Milford, January, 1891. 
RAPTORES.

FALCONES.

\section{FALCONIDAE.}

MAss.- Rare fall and winter visitor (four records). Oct.Mar. 11.

R. I.- Rare fall and winter visitor: Conanicut Id., Nor. 22, 1891; Newport, Oct. 28, 1896; Tiverton, Dec. 26, 1896.

200. Falco peregrinus anatum (Bonaparte) Blasius.

Duck hawk; American peregrine falcon; Black hawk (Vt.); Bullet hawk; Great-footed hawk; Ledge hawk.

Chapman, Handb. birds east. No. Amer., 1595, p. 209; A. K. Fisher, Hawks and owls of U. S., 1893, pl. 15. Egg, Bendire, 1S92, vol. 1, pl. 10, fig. 5-7.

Falco anatum Bonap., Geogr. and comparative list birds Europe and No. Amer., 1S3S, p. 4. "Northers parts" of No. America.

Open and mountainous country; nests on ledges or in cavities of large trees.

ME.- Rare summer resident. Mar. 14-Oct. Eggs, April 14.

N. H., VT.- Rare migrant and local summer resident. Mar. 14Oet. Vт., Eggs, May 2-June 30.

MAss.-Rare migrant, and in western mountains, rare local summer resident; rare in winter. Eggs, April 15-1Iay 23.

R. I.- Rare migrant. May; Oct.

Conv- - Rare migrant, summer and winter resident. Eggs, May 9 (large young, May 25, Talcott Mt.).

201. Falco columbarius Linné.

\section{Pigeon hawk.}

Linné, Sỵst. nat., ed. 10, 175s, vol. 1, p. 90. "America." Chapman, Handb. birds east. No. Amer., 1895, p. 210; A. K. Fisher, Hawks and owls of U.S., 1893, pl. 16. Egg, Bendire, 1892, vol. 1 , pl. 10 , figs. 4,8 .

Woods and open country; nests in low trees. 
RAPTORES.

FALCONES.

FALCONIDAE.

ME.- Cncommon migrant, rare in winter and summer; probably breeds in northern comties (fledglings noted, Knight). Mar. 2S-May 13; (summer ; Sept.-Oet. 10 (Dec. 1, 1906, Westbrook; Feb. 22, 1907, Scarborough).

N. H.-Uncommon migrant. May; Aug.-Sept.

VT.- Uneommon migrant; doubtfully recorded in summer.

Mass. - Uncommon migrant and rare winter resident. April 10-Mar 16; Sept. 7-Nov. 24; winter.

R. I.- Uneommon migrant, mainly in fall, and rare winter resident. April 24-May; Sept. 3-Nor. 21 (Jan.).

Cons.- Uncommon migrant and rare winter resident; doubtfully recorded in summer. April; (? Aug.); Sept. 25-Nor.; winter.

202. Falco tinxunculus Linné.

Kestrel.

Linné, Sysst. nat., ed. 10, 1758, vol. 1, p. 90. "IN Europae TurRibus." Dresser, Birds of Europe, 1S71-81, rol. 6, p. [113], pl. [384]. Egg, Seebohm, 1896, p. 7, pl. 4, fig. 5.

Open and wooded country; nests in dense woods, on cliffs, or in towers of buildings.

Mass.- Accidental risitor from Old World: Nantasket Beach, Sept. 29, 1889.

203. Falco sparverius Linné.

\section{American sparrow hawk; Killyhawk; Pigeon hawk.}

Limné, Srst. nat., ed. 10, 1758, vol. 1, p.90. “America." Chapman, Handb. birds east. No. Amer., 1895, p. 211; A. K. Fisher, Hawks and owls of C. S., 1893, pl. 17. Egg, Bendire, 1892, vol. 1, pl. 10, fig. 11-16.

Open country; nests in carities of trees or rarely of buildings.

ME. - Lncommon migrant and summer resident; rare winter 
resident in southern part (Portland, 1907-0S; Yarmouth, .Jan. 10, 1904). Mar. 19-Oct. 15 (winter). Eggs, Mayy 30.

N. H.- Uncommon migrant and rare summer resident north to White Mt. valleys. Mar. 15-Oct. Eggrs, May 16.

V'T.- Lncommon migrant and summer resident. Mar. 21-fall.

MAss.- Uncommon migrant and summer resident; uncommon winter resident near coast. Eggs, April 27-May 26.

R. I.- Uneommon migrant, rare winter and summer resident. Eggs, May 10.

Conn.- Uneommon resident. Eggs, April 26-May 7.

204. Pandiox hallätus Carolnnensis (Gmelin) Ridgway.

\section{American osprey; Fish hawk.}

Chapman, Handb. birds east. No. Amer., 1895, p. 212; A. K. Fisher, Hawks and owls of U. S., 1893, pl. 1S. Egg, Bendire, 1892, vol. 1, pl. 10, fig. 17; pl. 11, fig. 5-9.

Falco haliaëtos $\gamma$. carolinensis Gmel., Syst. nat., 17SS, vol. 1, pt. 1, p. 263. "AMERICA."

Seacoasts and vicinity of large streams and lakes; nests in high or low trees, on rocks or artificial sites.

ME.- Common summer resident. (Mar. 2S) April 5-Oct. 15. Eggs, May S-June 19.

N. H.- Lncommon migrant; formerly bred at Lake Lmbagog. April; Aug.-Nor. 1.

VT.- Lncommon migrant.

MAss.-Common migrant, and in Bristol Co. a summer resident. (Mar. 16) April 2-May 29; summer; Aug. 26-Nor. 11. Eggrs, May S-July.

R. I.-Common migrant and summer resident coastwise. Mar. 2t-Oct. 21 . Eggs, May 2-24.

Coxs.-Common migrant and uncommon summer resident coastwise. Mar. 20-Nor. 13 (Dec. 1). Eggs, May 5-July 4. 
RAPTORES.

STRIGES.

\section{ALUCONIDAE.}

205. Aluco pratincola (Bonaparte) A. O. U. comm.

\section{American barn owl; Monkey-faced owl.}

Strix pratincola Bonap., Geogr. and comparative list birds Europe and No. Amer., 1S38, p. 7. "Northers parts" of No. America. Chapman, Handb. birds east. No. Amer., 1895, p. 213; A. K. Fisher, Hawks and owls of U. S., 1893, pl. 19. Egg, Bendire, 1S92, vol. 1, pl. 12, fig. 1.

Open country, hiding in hollow trees and cavities during the day; nests in hollow trees, burrows, cavitics in cliffs or buildings.

ME.- Erroneously recorled from Falmouth.

VT.- Accidental visitor: Danby, 1902; Lyndon, June 4, 1894. Mass.- Rare visitor. May-Dec.

R. I.- Rare visitor; five records.

Coxs.- Rare visitor. June 11-Oct. 28.

\section{STRIGIDAE.}

\section{Asio wilsonianes (Lesson) Coues.}

\section{American long-eared owl; Cat owl.}

Chapman, Handb. birds east. No. Amer. 1595, p. 214; A. K. Fisher, Hawks and owls of U.S., 1893, pl. 20. Egg, Bendire, 1892, vol. 1, pl. 1, fig. 2.

Otus wilsonianus Less., Traité d'ornith., vol. 1, 1S31, p. 110. "LES ÉTATS-CNis. . . PENSYlvaNie . . . New-York."

Dense woods and swamps; nests in trees, usually in old crow or hawk nests.

Me., N.H., Iт.- Uncommon resident. Eggs, May 1.

MAss. - Uncommon fall and winter visitor, rare summer resident. Eggs, April 1-May 14.

R. I.- Rare resident. Eggs, April 10.

Conx.- Uncommon resident. Eggs, early April-April 25. 
RAPTORES.

STRIGIDAE.

207. Aslo flamieus (Pontoppidan) A. O. U. comm.

Short-eared owl; Bog owl; Gray marsh owl; Marsh owl.

Strix flammea Pontopp., Danske atlas, 1763, vol. 1, p. 617, pl. 25. Based on Linné: [Sweden].

Asio accipitrimus (Pallas). Clapman, Handb. birds east. No. Amer., 1S95, p. 215; A. K. Fisher, Hawks and owls of U. S., 1S93, pl. 21. Egg, Bendire, 1S92, vol. 1, pl. 12, fig. 3.

Open country and marshes; nests on the ground.

Me.- Encommon resident, more common migrant near coast.

N. H.- Uncommon migrant. April; Nor.

VT.- Uncommon migrant (? winters).

MAss. - Uncommon migrant mainly near coast, rare in winter; raresummer resident. Mar. 15-April 15; summer; Sept. 24-Nor. 30 (winter).

R. I.- Uncommon migrant and rare summer resident; may winter rarely. April; Oct. 26-Dec. 23. Eggs, April $2 S$.

Coxs.- Uneommon migrant; rare summer and winter resident.

20S. STRIX varia Barton.

\section{Barred owl; Barn owl (Me.); Black-eyed owl; Hoot owl; Round-headed owl.}

Strix varius Barton, Fragm. nat. hist. Pemna, 1799, p. 11. "Pexssyluasta."

Symnium nebulosum (Forst.). Chapman, Handb. birds east. No. Amer., 1895, P. 215; A. K. Fisher, Hawks and owls of U. S., 1S93, pl. 22. Egg, Bendire, 1S92, vol. 1, pl. 12, fig. 4.

Heary woods, preferably near water; nests in hollows of trees or in abandoned hawk or erow nests.

ME.- Rather common resident. Eggs, Mar. 27.

N. H.- Rather common resident up to 3000 feet.

VT. - Rather common resident. 
RAPTORES.

STRIGES.

STRIGIDAE.

Mass.- Uncommon resident, more frequent in fall migration. Egggs, Mar, 27-May 13.

R. I.- Uncommon resident, occasionally more common in fall and winter. Eggs, Mar. 15-April 12.

Coxx.-Uncommon resident. Eggs (Feb.), Mar. 1-April 30 (May 10).

209. Scotiaptex nebulosa (Forster) Preble.

\section{Great gray owl; Cinereous owl; Spectral owl.}

Strix nebulosa Forst., Phil. trans. ror. soc. London, 1772, rol. 62, p. 424. "Circa Sivur Hudsoxis."

Strix cinerea Gmel. Swainson and Richardson, Fauna BorealiAmer., 1831, vol. 2, p. 77, pl. 31. Egg, Bendire, 1892, vol. 1, pl. 12, fig. 5 .

Wooded country; nests in trees.

ME.- Rare and irregular winter visitor. Nor. S-Mar. 2.

‥ H.- Rare winter visitor.

VT.-Rare and irregular winter visitor. Nor. 27-Feb. 11.

MAss. - Rare winter visitor. Nor. 10-Mar. 4.

R. I.- Tery rare winter visitor: Fox Island, Mar. 25, 1883; near Providence, 1870, Nov. 19, 1906.

Coxx.-Rare winter visitor: North Haren, Jan. 18, 1893; Stratford, Jan. 6, $18 \pm 3$.

210. Glati funerea richardsoni (Bonaparte) A. O. U. comm.

Richardson's owl; Sparrow owl.

Nyctale richardsoni Bonap., Geogr. and comparative list birds Europe and No. Amer., 183S, p. 7. Based on Audubon: "AT Bangor, in Mane, on the Pexobscot River."

Strix tengmalmi Limné. Swainson and Richardson, Fauna Borcali-Amer., 1831, vol. 2, p. 94, pl. 32. Egg, Bendire, 1S92, vol. 1, pl. 12, fig. 6 . 
RAP'TORES.

STRIGES.

\section{STRIGIDAE.}

Wooded country; nests in holes in trees.

ME.- Winter visitor of irregular abundance. (Sept.) Nov. 30Mar. 16.

N. H.- Rare winter visitor. Dee. 15-Mar. 1.

VT. - Rare winter visitor.

Mass. - Rare winter visitor. Nov. 26-Mar.

R. I.- Rare winter visitor (four records). Dec. 18.

Conv.- Rare winter visitor: Eist Windsor, 1859.

211. Glaux aCAdicus (Gmelin) A. O. U. comm.

\section{Saw-whet owl; Acadian owl; Little owl.}

Strix acadica Gmel., Syst. nat., 17SS, vol. 1, pt. 1, p. 296. "IN America septentrionali."

Nyctala acadica (Gmel.). Chapman, Handb. birds east. No. Amer., 1895, p. 217.

Ulula acadica (Gmel.). Audubon, Birds of Amer., 1St0, vol. 1, p. 123, pl. 33. Eger, Bendire, 1892, vol. 1, pl. 12, fig. 7.

Dense roods; nests in old woodpecker holes or carities of trees.

Me., N. H.- Uncommon resident. Eggrs, April 6.

TT.- Uncommon resident, mainly in northern part.

MASS.- Uncommon resident, breeding locally. Egges, April 4 May 1 (July 3).

R. I.- Uncommon winter visitor; may breed rarely. Nov.Feb.

Cons.- Rare resident.

212. Otus asio (Linné) Stone.

Screech owl; Cat owl; Little horned owl; Mottled owl; Red owl.

Strix asio Linné, Syst. nat, ed. 10, 175S, vol. 1, p. 92. "AnIEnICA." 
RAPTORES.

STRIGES.

\section{STRIGIDAE.}

Megascops asio (Linné). Chapman, Handb. birds east. No. Amer., 1895, p. 21S; A. K. Fisher, Hawks and owls of U. S., 1S93, pl. 23. Egg, Bendire, 1S92, vol. 1, pl. 12, fig. 8 .

Open woods, orchards, and shade trees; nests in cavities of trees.

ME.-- Rare resident.

N. H.- Uncommon resident of southern part, becoming rare in southern valleys of White Mts.

$V_{T}$.- Uncommon resident at lower elevations.

Mass.-Common resident. Eggs, April 15-May 5.

R. I.-Common resident. Eggs, April 7-May 2.

Coxs.- Common resident. Eggs, April 7-26.

213. Bubo virginianus (Gmelin) Bonaparte.

\section{Great horned owl; Cat owl; Eagle owl; Hoot owl.}

Chapman, Handb. birds east. No. Amer., 1895, p. 219; A. K. Fisher, Hawks and owls of U. S., 1S93, pl. 24. Egg, Bendire, 1892, vol. 1, pl. 12, fig. 12 .

Strix virginiana Gmel., Syst. nat., 17SS, vol. 1, pt. 1, p. 287. "in omni America, in Astracania ad Camischatcan usque, ET POLO ARCTICO PROPIUS."

Heary woods; nests in carities of trees, in open nests on the branches, or rarely on the ground.

Me., N. H.- Uncommon resident. Egggs, Mar. 11-23.

$\mathrm{V}_{\mathrm{T}}$.- - Uncommon resident.

Mass.- Uncommon resident. Eggs, Feb. 17-April 20.

R. I.- Rare resident. Eggs, Feb. 28-Mar. 11.

Coxx.- Uneommon resident. Eggs, Feb. 19-Mar. S (April 12, second lạing).

214. Bubo virginianus subarcticus (Hoy) Ridgway.

Arctic horned owl.

Bubo subarcticus Hay, Proc. acad. nat. sci. Phila., 1852, vol. 6, p. 211. "Wisconsin." 
RAPTORES.

STRIGES.

STRIGIDAE.

Strix aretiea Swains. Swains. and Richardson, Fanna BorealiAmer., 1S31, vol. 2, p. S7, p). 32.

Heary woods or open comtry; nests probably in cavities of trees, ol on their branches.

Mr.- Aecidental visitor: "Maine"; Brownfield, 1 SS6. (Knight supposes these to be "pale or faded individuals of the typical Horned Owl.")

MAss. - Accidental visitor: Waltham, Nov. 30, 1867.

215. Bubo virginianus heterocnemis (Oberholser) A. O. U. comm.

\section{Labrador horned owl.}

Asio magellanieus heterocnemis Oberh., Proc. U. S. nat. mus., 1904, vol. 27, p. 187 "LANCE AU Loup, LABrador."

Dense forests; nests in trees.

ME - Accidental visitor: near Portland, Mar. 1870 (recorded as B. v. saturatus).

216. Nyctea ryctea (Linné) Lichtenstein.

\section{Snowy owl; Arctic owl; White owl.}

Strix myctea Limné, Syst. nat., ed. 10, 175S, rol. 1, p. 93 . "IN Europa \& Anerica septentrionali."

Surnia nyetea (Linné). Audubon, Birds of Amer., 1S40, vol. 1, p. 113, pl.2S. Egg, Bendire, 1S92, vol. 1, pl. 12, fig. 19.

Open countryand barren grounds; nests on the ground.

ME.- Uncommon fall and winter visitor. (Sept.) Oct. 15Feb.

N. H.- Late fall and winter visitor, sometimes common; aecidental in summer (Concord, July). Oct. 10-April (July).

VT.- Irregular fall and winter visitor. Oct. 20.

MAss.- Irregular fall and winter visitor, sometimes common. (Oct. 12) Oct. 2S-April 15 (May 20). 
RAPTORES.

STRIGES.

STRIGIDAE.

R. I.- Uncommon and irregular winter visitor. Sov. 3-Feb. 15.

Coxs.- Uncommon and irregular winter visitor. Oct. 17.

217. Survia Ulula Caparoch (Müller) Stejneger.

\section{American hawk owl; Day owl.}

Strix caparoch Müll., Linné Vollstand. natursyst., suppl., 1776, p. 69. "Europa."

Sumia funerea Gmel. Audubon, Birds of Amer., 1St0, vol. 1, p. 112, pl. 27. Egg, Bendire, 1892, vol. 1, pl. 12, fig. 18.

Open country or woods; nests in trees or rarely in cliffs.

Me.-Rare fall and winter visitor. Oct. 24-Mar. 10 (April 16, 1906, Van Buren).

N. H.- Rare fall and winter visitor. Oct. 25-(April 20, Connectieut Lakes).

YT.-Rare fall and winter risitor; (? summer in north). Nor. 2-winter.

MAss.- Rare fall and winter visitor. Nor. 16-Mar. 25.

R. I.- Rare winter visitor: West Greenwich, Nor. 16, 1906; (also one doubtful record).

Coxx-- Rare winter visitor: New Haven, Nov. 1869.

218. Speotyto cunicularia hypgata (Bonaparte) Coues.

\section{Burrowing owl.}

A. K. Fisher, Hawks and owls of U. S., 1893, p. 195, pl. 25. Egg, Bendire, 1892, vol. 1, pl. 12, fig. 14.

Strix hypogace Bonap., Amer. ornith., 1825, vol. 1, p. 72, pl. 7 , fig. 2. "Western Anerichn territory."

Open prairie country; nests in old burrows of prairie-dogs and spermophiles.

Mass-Aecidental visitor: Newburyport, May 5, 1875. 
COCCIGES.

CLCULI.

CUCULIDAE.

219. Coccrzus americanus (Linné) Bomaparte.

Yellow-billed cuckoo; Chow-chow; Egg sucker; Milk sourer; Rain crow; Rain dove.

Audubon, Birds of Amer., 1S42, vol. 4, p. 293, pl. 275. Egg, Bendire, 1895, vol. 2, pl. 5, fig. 1.

Cuculus americamus Linné, Sỵst. nat., ed. 10, 175S, vol. 1, p. 111. "Carolina."

Thiekets, shade trees, and open woods; nests in low trees or bushes.

ME.- Rare summer resident in southern counties. May 1SAug.

N. H.- Rare summer resident of coast and southern valley's. May-Sept.

VT.-Rare summer resident at lower elevations. May-summer. Eggs, June 4-July 10.

MAss. - Uneommon summer resident, mainly of south and east parts. (May 4) May 9-Sept. 26 (Oct. 6; Nor.). Egrs, May 20June 30 .

R. I.-Summer resident, sometimes common. May 9-Sept. 25 (Oet. 23). Eggrs, May 24-30.

Coxx-Common summer resident. May 7-Sept. (Oet. 11). Egggs, June 1-July 24 (Aug. 4, 1S, 26, Sept. 23).

220. Coccizts erithrophthalits (Wilson) Bonaparte.

Black-billed cuckoo; Chow-chow; Egg sucker; Milk sourer; Rain crow; Rain dove.

Audubon, Birds of Amer., 1842, vol. 4, p. 300, pl. 276 . Egg, Bendire, 1595, vol. 2, pl. 5, figs. 3, 4.

Cuculus erythropthalma Wils., Aner. ornith., 1S11, vol. 4, p. 16, pl. 28. "Georgia."

Thickets, shade trees, and open woods; nests in low trees or bushes. 
COCCYGES.

CUCULI.

CUCULIDAE.

Me.-Common summer resident. May 11-Sept. 14. Eggs, June 17.

N. H.- Common summer resident at lower elevations. May 12Sept. 22 (Oct. 5).

VT.-Common summer resident. Eggs, (Aug. 13).

MAss.-Common summer resident. May 4-Sept. 27 (Oct. 21). Eggs, May 20-June 17.

R. I.-Common summer resident. May 11-Sept. 16. Eggs, May 27-June 1.

Coxx.-Common summer resident. May 15-Sept. 28 (Nov. 28, 1Sss, Stratford). Eggs, June 7-July 24 (Aug. 26).

ALCYONES.

\section{ALCEDINIDAE.}

221. Ceryle alcron (Limné) Bomaparte.

\section{Belted kingfisher; Kingfisher; Lazy-bird.}

Alcedo alcyon Linné, Sỵst. nat., ed. 10, 1758, vol. 1, p. 115. "Anerica." Audubon, Birds of Amer., 1S42, vol. 4, p. 205, pl. 255. Egg, Bendire, 1895, vol. 2, pl. 1, fig. 3.

Vicinity of bodies of water; nests in burrows excarated in banks.

Me.-Common summer resident. April 12-Sept. (Dec. 27). Eggs, Jume 1.

N. H.-Common summer resident; rare winter resident near open streams. Mar.-Nov, 24 (winter). Eggs, May 20.

VT.-Common summer resident; rare winter resident. Mar. 27-Oct. (winter, Brattleboro).

MAss.-Common summer resident, and rare winter resident. Mar. 15-Dec. 5 (winter). Eggs, May 15-June 6.

R. I.-Common summer and uncommon winter resident. Mar.-Oet. 20; winter. Eggs, May 15-20.

Coss.-Common summer and uncommon winter resident. Milr. 29-Nov. 13; winter. Eggs, May 14. 
PICI.

\section{PICIDAE.}

222. Dryobates villosts (Limné) Cabanis.

\section{Hairy woodpecker; Big guinea woodpecker; Big sapsucker.}

Picus villosus Linné, Syst. nat., ed. 12, 1766, vol. 1, p. 175. "Ix Auerica septextrioxili." Audubon, Birds of Amer., 18t2, rol. 4, p. 24t, pl. 262. Egg, Capen, 1856, pl. t, fig. 9.

Forests; nests in holes dug in dead or live trees.

ME.-Common resident. Eggs, May $2 S$.

N. H. - Common resident up to $\$ 500 \mathrm{ft}$.

VT. - Uncommon resident. Egrgs, Jume $S$.

MAss. - Uncommon winter visitor and less common summer resident, especially in western part. Eggs, April 22-May 30.

R. I.- Uncommon fall and winter risitor and rare summer resident (Chepachet, Cranston). Sept. 1.-Mar.; summer. Eggs, May 12-28.

Coxs.- Uncommon fall and winter visitor, rare summer resident. Eggs, May 2-9.

223. Dryobates pubescexs mediayes (Swainson) Brewster.

\section{Downy woodpecker; Little guinea woodpecker; Little sap- sucker.}

Picus (Dendrocopus) medianus Swains., in Swains. and Richardson, Fauna Boreali-Amer., 1S31, rol. 2, p) 30S. "IIDDLE PARTS of North America.... New Jersey."

Dryobates pubescens (Linné). Chapman, Handb. birds east. No. Amer., 1595, p. 230; Bird-life, 1S9S, pl. 24. Egg. Capen, 1SS6, pl. 14, fig. 10.

Open woods, shade and orchard trees; nests in luoles excavated in dead trees.

ME.-Common resident. Egggs, May 27.

N. H.-Common resident, mainly below $3000 \mathrm{ft}$.

VT.-Common resident. 
PICI.

\section{PICIDAE.}

MAss.- Common resident, most conspicuous in fall and winter. Eggs, May 20-June 21.

R. I.- Uncommon summer and common winter resident. Eggs, May 12-25.

Conn.- Common resident.

224. Picoldes arcticus (Swainson) Gray.

Arctic three-toed woodpecker; Black-backed three-toed woodpecker.

Pieus (Apternus) areticus Swains., in Swains. and Richardson, Fauna Boreali-Amer., 1S31, vol. 2, p. 313, pl. 57. "NEAR THE sources of the Athabasca River, lat. 57\%" Egg, Capen, 1SS6, pl. 14, fig. 11.

Evergreen forests; nests in holes excavated in dead trees.

ME.- Uncommon winter visitor, and in northern and western counties, rare resident. Eggs, May 23.

N. H.- Rare resident above $3000 \mathrm{ft}$. in White $\mathrm{MIts}$, south to Mt. Passaconaway and possibly on Mt. Monadnoek (Keene, breeding), elsewhere rare visitor.

- VT.- Rare resident in northern part and on higher peaks (nests at Burlington, Lumenburg, Sherburnc, Pico Peak, etc.); occasional visitor to lower country in fall and winter. Eggs, May 29, June 1.

MAss.-Occasional fall and winter visitor. (Late Sept.) Oct. 16-April 27 (July, 1885, Boxford; Aug. 11, 1883, Dorchester).

Conx.- Rare winter visitor: East Windsor Hill; Simsbury, 1860 .

225. Pịcones aliericanus Brehm.

American three-toed woodpecker; Banded-backed woodpecker; Ladder-backed woodpecker.

Picoides americana Brehm, Handb. d. naturgesclı. aller vögel Deutschl., 1831, p. 195. "Anerika." 
PICI.

PICIDAE.

Picus hirsutus Vieill. Audubon, Birds of Amer., 1S42, rol. 4, p. 268, pl. 269. Egg, Capen, 18s6, pl. 14, fig. 12.

Evergreen forests; nests in holes excarated in dead trees.

ME. - Rare winter visitor, and in north and west, a rare resident. Eggs, June 2.

N. H. - Rare resident above $3000 \mathrm{ft}$, south to Sandwich range; elsewhere rare winter risitor. Eggs, June 4.

IT.- Rare winter visitor and local resident (Pico Peak above $2500 \mathrm{ft}$.).

MAss.- Rare winter visitor. April $2 S$.

226. Sphyrapicus varius (Linné) Baird.

Yellow-bellied sapsucker; Red-throated sapsucker; Squealing or Whining woodpecker.

Picus varius Linné, Syst. nat., ed. 12, 1766, vol. 1, p. 176. "IN America septentrionali." DeKay, Zool. New Iork, 1844, vol. 2, p. 1SS, pl. 1S, fig. 3S; Bonaparte, Amer. omith., 1825, rol. 1, pl. S, figs. 1, 2. Egg, Capen, 1SS6, pl. 14, fig. 13.

Woods, orchards, and shade trees; nests in holes excavated in dead trees.

ME.-Common migrant and in northern counties a common summer resident. (Mar. 8 and 31) April 10 - Oct. 26 (Nor. 15). Eggs, May 15-June 8 .

N. H. - Common migrant and in northern half a common summer resident up to $3000 \mathrm{ft}$; accidental winter resident (Charlestown, 1S9S-99). April 14-Oct. 20 (winter).

VT.-Common migrant, less common summer resident.

MAss.- Uncommon migrant and accidental winter resident; in Berkshire Co. a rare summer resident. April 4-Xay 10; summer; Sept. 10-Nov. 1 (winter).

R. I.-Uncommon migrant. (Mar. 23)-April; Sept. 26-Oct. 11.

Conn.- Rare spring and uncommon fall migrant. Mar. 30May 3; Sept. 2S-Oct. (Dec. 21, 190S, New Haven). 
PICI.

PICIDAE.

227. Phloeotomus pileatus abieticola (Bangs) A. O. U. comm.

Northern pileated woodpecker; Black woodpecker; Cock of the woods; Laughing woodpecker; Logcock; Stump-breaker; Woodchuck; Woodcock; Woodhen.

Ceophloeus pileatus abieticola Bangs, Auk, 1S9S, vol. 15, p. 176. "Greenvilile, Maine."

Picus pileatus Linné. DeKay, Zool. New York, 1S44, rol. 2, pl. 18, fig. 35. Eggr, Capen, 1886, pl. 14, fig. 6.

Heary timber; nests in holes exeavated in dead trees.

ME.- Uncommon resident in wilder portions. Eggs, May 11May 28.

N. H. - Rare resident mainly in northern and western parts.

VT.- Rare resident, breeds south to Taftsville, 'Townshend, Wells River. Eggs, May 22.

Mass.- Rare resident in western counties; elsewhere casual. Eggs, May 17.

Coxx. - Rare or now an aceidental visitor (Granby, Nov. 1, 1890, 1894-95; Iitehfield, Jume 20, 1908).

228. MIelanerpes erythrocephalus (Linné) Swainson.

\section{Red-headed woodpecker.}

Picus erythrocephalus Linné, Syst. nat., ed. 10, 1758, vol. 1, p. 113. "Alerica." Audubon, Birds of Amer., 18+2, vol. 4, p. 274, pl. 271. Egg, Capen, 1886, pl. 14, fig. 14.

Open woodlands and shade trees; nests in holes excarated in dead trees.

Me.-Occasional visitor, mainly in spring and fall; said to have bred in York Co.

N. H.- Rare and irregular visitor in southern half. May 25Sept.

TT.- Irregular visitor at all seasons, sometimes common; resident and bred in western part about 1880 (Orwell, Rutland). 
PICI.

\section{PICIDAE.}

MAss. - Rare irregular visitor at all seasons, sometimes breeding (Agawam, Brookline, Newton). Eggs, May-June 17.

R. I.-Irregular visitor, sometimes common in fall; rare summer resident (Providence Co.; Warwick, young in nest, July 2S, 1SS2). Eggs, May $2 S$.

Cons.- Rare summer resident and irregular visitor at all seasons; formerly more eommon.

229. Centurus carolinus (Limné) Bonaparte.

\section{Red-bellied woodpecker.}

Picus carolinus Iinné, Syst. nat., ed. 10, 1758, vol. 1, p. 113. "in America septentrionali." DeKay, Zool. New Tork, 1S44, vol. 2 , p. 189 , pl. 17 , fig. 37 .

Wooded bottomlands; nests in holes exeavated in dead trees.

Mass.-Accidental visitor (five records). May-Nor.

R. I.-Accidental visitor: near Providenee, two specimens.

Conn.-Aecidental visitor: Hartford; Stratford, Oet. 16, $18+2$. Suffield, July $30,187 t$.

230. Colaptes auratus luteus Bangs.

Northern flicker; Anteater; Clape; Golden-winged woodpecker; Grasshopper woodpecker ('t.); Harry wicket; High-hole; Pigeon woodpecker; Wake-up; Yaffle; Yellow jay; Yellowhammer; Yellow-wing (Mass.); Yucker.

Bangs, Auk, 189S, vol. 15, p. 177. "Watertown, MLass."

Colaptes auratus (Iinné). Chapman, Bird-life, 1898, p. 116, pl. 26. Egg, Calpen, 1S86, pl. 14, fig. 15.

Open woods, orchards, fields, clearings; nests in holes excavated in dead trees, or in cavities.

Me.- Common summer, and near coast, a rare winter resident. Mar. 11-Nov. 13 (winter). Eggrs, May 27-June 14. 
PICI.

\section{PICIDAE.}

N. H.- Common migrant and summer resident below $2000 \mathrm{ft}$; rare winter resident near coast. Eggs, May 24.

VT.-Common migrant and summer resident. April 18-fall.

Mass.-Common migrant and summer resident; less common winter resident, mainly near coast. Eggs, May 6-June 20.

R. I.-Common migrant, less common summer and winter resident. Mar. 1S-Nor.; winter. Eggs, April 29-May 19.

Cons.-Common migrant and summer resident; less common winter resident. Mar. 2S-Nov. 2S; winter. Eggs, May 1SJune 9.

MACROCHIRES.

CAPRIMULGI.

\section{CAPRIMULGIDAE.}

231. Antrostomus CArolinensis (Gmelin) Gould.

\section{Chuck-will's-widow.}

Caprimulgus carolinensis Gmel., Syst. nat., 17SS, rol. 1, pt. 2, p. 102S. "Yirginia \& Carolixa." Audubon, Birds of Amer., 1S40, rol. 1, p. 151, pl. 41. Egg, Bendire, 1S95, rol. 2, pl. 1, figs. S, 9.

Woods and thickets, active at night; nests on the ground.

MAss.-Accidental visitor: Revere (Oct. or Dec.), $18 S t$.

Cons.- Accidental visitor: New Haven, May 19, 1859 (after southerly storm).

232. Antrostomus vociferus (Tilson) Bonaparte.

Whip-poor-will.

Caprimulgus vociferus Wils., Amer. ornith., 1812, vol. 5, p. 71, pl. 41, fig. 1-3. "Kentuckr." Audubon, Birds of Amer., 1S 40 , vol. 1, p. 155, pl. 42. Egg, Bendire, 1S95, vol. 2, pl. 1, figs. 10, 11.

Woods and thickets, active at night; nests on the ground.

ME.-Common summer resident. April 2S-Sept. 21. 
CAPRIMULGIDAE.

․ H.- Common summer resident north to White Mt. valleys. Maly 6-Sept. 26.

TT.-Common summer resident at lower elevations. May 5Sept. 16. Eggs, May 2t-31.

Mass.-Common summer resident. (April 22) April 30-Sept. 25. Lgggs, May 18-June 9.

R. I.-Common summer resident. April 25-fall. Eggs, May 25-June $S$.

Coxs.- Common summer resident. Late April-Sept. (Nov. 1, 1850, Noank). Eggs, May 20-June 5 (July 4).

\section{Chordeiles virgivianus (Gmelin) Swainson.}

\section{Nighthawk; Bull-bat; Mosquito hawk; Pork-and-beans.}

Audubon, Birds of Amer., 18t0, vol. 1, p. 159, pl. 43. Egg, Bendire, 1595, vol. 2, pl. 2, fig. 1-3.

Caprimulgus virginianus Gmel., Syst. nat., 178S, vol. 1, pt. 2, p. 102S. "In Anerica septentrionali, praesertim Virginia."

Woods, thickets, or in cities and open country; nests on the ground or on flat gravelled roofs; largely noctumal.

ME.-Common migrant and summer resident. (April 11, East Hebron; April 22) May 3-Sept. 14. Eggs, June 26-July 4.

N. H.- Common migrant and at lower altitudes a less common summer resident. (April 17) May 9-Sept. 15. Eggrs, June 10.

VT.-Common migrant and summer resident. May-Sept.

MAss.-Common migrant, less common summer resident. (April 23) May 9-Oct. 6. Eggrs, June 5-15.

R. I.-Common migrant (mainly inland), and summer resident. (April 7) Maty-Sept. (Oct.) Eggs, June 5-8.

Coxi- Common migrant and less common summer resident. May 11-Oet. Eggs, June 3-23. 
MACROCHIRES.

CYPSELI.

MICROPODIDAE.

234. Chaetura pelagica (Limé) Stephens.

\section{Chimney swift; Chimney-bird; Chimney swallow.}

Chapman, Handb. birds east. No. Amer., 1895, p. 239; Birdlife, 1S9S, pl. 2S. Egg, Bendire, 1895, vol. 2, pl. 1, fig. 25.

Hirundo pelagica Linné, Syst. nat., ed. 10, 175S, vol. 1, p. 192. "Anerica."

Lives largely on the wing; rests and nests in hollow trees, chimneys, or barns.

ME.-Common summer resident. April 26-Sept. 11. Eggs, June 15-July 10.

N. H.-Common summer resident. April 27-Sept. 7 (Oct. 1).

VT.-Common summer resident. May 1-Sept.

Mass.-Common summer resident. (April 1S) April 25-Sept. 25 (Oct. 12, 1907, Sharon). Eggs, June 10-30.

R. I.-Common summer resident. April 26-Sept. 17. Eggos, Jume 10-July 1.

Coss.-Common summer resident. (April 14) April 21-Sept. 27. Eggs, June 24.

TROCHILI.

TROCHILIDAE.

235. Trochllus colubris Linné.

Ruby-throated hummingbird.

Linné, Syst. nat., ed. 10, 1758, vol. 1, p. 120. "Ix AmericA, mprimis septentrionali." DeKay, Zool. New Tork, 1S41, vol. 21, p. 46, pl. 40, fig. \$7. Egg, Bendire, 1595, vol. 2, pl. 1, fig. 27.

Open country, launting flower growths; nests in trees.

ME.-Common summer resident. May 6-Sept. 26 (Oet. 1).

X. H.- Common migrant, less common summer resident at lower elevations. May 13-Sept. 25. Eggs, June 29-July 15.

VT.- Uncommon summer resident. May 15-Sept. 
TROCHILIDAE.

Mass-Common migrant, less common summer resident. (May 1) May 6-Sept. 23 (Oct. 15). Eggss, May 24-June 15.

R. I.-Common summer resident. (May 3) May 6-Sept. 23. Eggs, May 20-July 4.

Cosx.-Common summer resident. May 5-Oct. 5 (Oct. 16). Eggs, May 27-June 3 (Aug. 4).

PASSERES.

CLAMATORES.

TYRANNIDAE.

236. Muscivora forlicata (Gmelin) Oberholser.

\section{Scissor-tailed flycatcher.}

Muscicapa forficata Gmel., Syst. nat., 178S, vol. 1, pt. 2, p. 931. "IN Nova Hispania." Bonaparte, Amer. ornith., 1825, vol. 1, p. 15, pl. 2, fig. 1. Egg, Bendire, 1895, vol. 2, pl. 1, figs. 12, 13.

Open tree-grown country; nests in trees.

TT.-Aceidental visitor: St. Johnsbury, ? 1884.

Coxs.-Aecilental visitor: Wauregan, about April 27, 1876.

237. 'Tyrannus trrannus (Limné) Jordan.

\section{Kingbird.}

Chapman, Handb. birds east. No. Amer., 1595, p. 243; Birdlife, 189S, pl. 30. Egg, Bendire, 1S95, vol. 2, pl. 1, figs. 14, 15.

Lanius tyranmus Linné, Syst. nat., ed. 10, 1758, vol. 1, p. 94. "in Airerica septentrionali."

Orchards and tree growth along streams and lakes; nests in trees.

Me.-Common summer resident. Mar 3-Sept. 12 (Sept. 25). Eggs, June 15.

N. H.-Common summer resident north to the White Mt. valleys. May $3-$ Sept. 11.

VT.-Common smmmer resident. Maly 5-Sept.

MAss.-Common summer resident. (April 27) May 3-Sept. 19 (Sept. 26). Eggs, May 30-July 4 (July 17). 
PASSERES.

CLAMATORES

TYRANNIDAE.

R. I.-Common summer resident. May 1-Sept. 12 (Sept. 28). Eggs, Nay 31-June 17.

Coxx-Common summer resident. (April 13, 26) May 1Sept. 15 .

238. Trrannus dominicensis (Gmelin) Richardson.

\section{Gray kingbird.}

Lanius tyranns $\beta$. dominicensis Gmel., Syst. nat., 17SS, vol. 1, pt. 1, p. 302. "insula S. Dominici et Jamaica."

Muscicapa dominicensis Brisson. Audubon, Birds of Amer., 1840, vol. 1, p. 201, pl. 55. Egg, Bendire, 1895, rol. 2, pl. 2, figs. 3,4 .

Tree growth along seacoasts and large streams; nests in low trees.

Mass.-Accidental visitor: Lynn, Oct. 23, 1869.

239. Trrannes verticalis Say.

\section{Arkansas kingbird.}

Say, Long's Exped. to Rocky Mts., 1823, vol. 2, p. 60. On the "ARKANSA" River.

Muscicapa verticalis (Say). Bonaparte, Amer. ornith., 1825, rol. 1, p. 18, pl. 2, fig. 2. Eggg, Bendire, 189.5, vol. 2, pl. 1, figs. 16, 17.

Open country, usually near water; nests in trees.

Me.-- Accidental visitor: Eliot, Oct., ? 1864.

240. Mytarchus crivitus (Linné) Lichtenstein.

\section{Crested flycatcher; Great crested flycatcher.}

Muscicapa crinita Limné, Syst. nat., ed. 12, 1766, vol. 1, p. 325. "in Averica septentrionali." Audubon, Birds of Amer., 1St0, vol. 1, p. 209, pl. 57. Egg, Bendire, 1S95, vol. 2, pl. 2, figs. S, 9.

Open woods; nests in hollow limbs. 
Me.- Uncommon summer resident, mainly in southern part. May S-Sept. 20. Eggs, Jume 11-25.

N. H.- Uncommon summer resident north to White MIt. valleys. May 13-Sept. Eggss, early June-July 7.

VT.-Common summer resident. May S-Sept.

MAss.- Uncommon summer resident. (May 1) May 9-Sept. 26 (Oct. 15). Eggs, June 13-27.

R. I.- Uncommon summer resident. May 2-Aug. 23. Eggs, June 5,6 .

Coxx.-Common summer resident. May 4-Sept. 17. Eggs, May 27-June 13.

241. Sayornis phoebe (Latham) Stejneger.

Phoebe; Barn, Bridge, or House pewee; Bridge-bird; Pewit.

Ridgway, Bull. 50, U. S. nat. mus., 1907, pt. 4, p. 594 . Egg, Bendire, 1895, vol. 2, pl. 1, fig. $2 S$.

Muscicapa phocbe Lath., Index ornith., 1790, vol. 2, p. 489. "in America septentrionali, Noveboraco."

Muscicapa fusca Bonap. Andubon, Ornith. biogr., 1834, vol. 2, p. 122 , pl. 120 .

Borders of streams and ponds, or about buildings; nests under bridges or eulverts, or on projections of buildings or ledges.

ME.-Common summer resident. Mar. 22-Oct. 19 (Nov. 24, 1SSt, Brewer). Eggrs, May 7-June 24.

X. H-Common summer resident at lower elevations. Mar. 22-Oct. 17. Egrgs, June 7-July.

VT.-Common summer resident. Mar. 22-fall.

Mass.-Common summer resident. (Feb. 20) Mar. 12-Oct. 26 (Nor. 15). Eggs, April 27-May 10; June 13-July 3 (2d brood); Aug. 15 (? 3d brood).

R. I.-Common summer resident. Mar. S-Oct. 27. Eggs, May $7-12$. 
PASSERES.

CLAMATORES.

\section{TYRANNIDAE.}

Cons.- Common summer and rare or accidental winter resident (New Haven, Dec. 21, 1906-Mar. 10, 1907; Dec. 25, 1907). (Feb. 25) Mar. 11-Oct. 30 (winter). Eggs, May 12-June 11; July 10 .

242. SAYORnis SAYA (Bonaparte) Baird.

\section{Say's phoebe.}

Muscicapa saya Bonap., Amer. ornith., 1525, vol. 1, p. 20, pl. 2, fig. 3. "NEAR THE ARKANSAW RIVER, ABOUT TWENTY MLES From The Rocki Mountains.” Egg, Bendire, 1895, vol. 2, pl. 1, fig. 29.

Open country; nests under bridges, projections of buildings and ledges.

MAss.--Accidental visitor: North 'Truro, Sept. 30, 1859.

243. Nuttallornis borealis (Swainson) Oberholser.

\section{Olive-sided flycatcher; Three-deer.}

Tyrannus borealis Swains., in Swains. and Richardson, Fauna Boreali-Amer., 1S31, vol. 2, p. 141, pl. 35. "Cumberland House, LAт. 54. "' Egg, Bendire, 1S95, vol. 2, pl. 2, figs. 15, 16.

Mixed and coniferous woods; nests in trees.

ME.- Uncommon summer resident, south to Cape Elizabeth. May 7-Sept. 15. Eggs, June 17-23.

N. H.- Uncommon summer resident. May 18-Sept. 14.

VT. - Uncommon summer resident, mainly of mountainous regions. Nay 10-fall. Eggs, June 10-16.

Mass. - Uncommon summer resident in western part, rare and local in the east. May 10-Sept. 10. Eggs, June 16-July.

R. I.- Rare migrant. May 14-24; ? fall.

Corv.- Rare migrant; may breed in northwestern part. Spring; Aug. 5-Oct. 1 S. 
PASSERES.

CLAMATORES.

TYRANNIDAE.

244. Mrochases riress (Limé) Ridgway.

Wood pewee; Dead-limb bird.

Muscicapa viren. Limné, Syst. nat., ed. 12, 1766, vol. 1, p. 327. "ix Carolixa AD Ripas." Aulubon, Birds of Amer., 1840, vol. 1, pl. 64 .

Contopus virens (Limé). ('hapman, Handb, birds east. No. Amer., 1895, p. 247. Egag, Bendire, 1895, vol, 2, pl. 2, figs. 18, 19.

Woods and shade trees; nests in trees.

Me.-Common summer resident. May 12-Sept. 27. Eggs, June 20.

N. H.-Common summer resident at lower elerations. May 17-Sept. 17. Egges, June 9-16.

VT.-Common summer resilent. May 19-Sept.

Mass-Common summer resident. (May 10) May 12-Sept. 26 (Oct. 1). Eggs, June 10-July 10.

R. I.-Common summer resident. May 12-Sept. 24. Eggs, June $15-30$.

Coxx-Common summer resident. Mary 10-Sept.

245. EMPIDONAX FLATIEATRIS (Baird) Baird.

\section{Yellow-bellied flycatcher.}

Tyrammia flarientris Baird, Proc. acarl. nat. sci. Phila., 1843, rol. 1, p. 20.3. "Neatr Carlasle, Pexvertuavia."

Muscicapa flariventris (Baird). Audubon, Birds of Amer., 1.54t, vol. 7, p. 341, pl. 490. Eigr, Bendire, 1895, vol. 2, pl. 2. fig. 23.

Damp erergreen thickets; nests among tree roots.

Me.- Lnemmons summer resident in northern part. May 21Jume 1; summer Sept. S. Eurgs, June 1t-27.

N. H.-Common summer resident above $3000 \mathrm{ft}$. from White Mts. north; local south to Dublin. May 1S-Auge. ("Sept.). Eggra, June 14 . 
PASSERES.

CLAMATORES.

TYRANNIDAE.

VT.- Uneommon local summer resident at lower elevations south to Londonderry. Mary 16-summer.

Mass. - Rare migrant and possible summer resident on Mt. Greylock (June 15, 1907). (May 10) May 15-June 10 (June 15); Aug. 25-Sept. 10 (Nov. 29, 1S76, Reading; Dee. 1, 1S76, Nerton).

R. I.- Rare migrant. May 21; (? July 27) Aug. 7.

Coxw- Cneommon migrant. May 20-early June; Sept. 17.

246. Empidonax virescens (Vieillot) Brewster.

\section{Green-crested flycatcher; Acadian flycatcher.}

Chapman, Handb. birds east. No. Amer., 1895, p. 249. Egg, Bendire, 1895, rol. 2, pl. 2, figs. 26, 27.

Platyrhynchos virescens Vieill., Nonr. diet. d'hist. nat., 181S, vol. 27, p. 22. No locality, based on Wilson.

Inscicapa acadica Gmel. Audubon, Birds of Amer., 1St0, vol. 1 , p. 221, pl. 62 .

Deep woods near water; nests, pensile, in low trees.

VT.- Rare summer resident: Bennington (nest with young, July 14,1904$)$; various doubtful records.

Mass. - Very rare summer resident (Hyde Park, 1sss; nec Springfield).

Cons.- Rare local summer resilent (Greenwich, Stamford, Suffield). Eggs, June 2-2.2.

247. Empidonax trailli alyordu Brewster.

\section{Alder flycatcher; Traill's flycatcher.}

Brewst., Auk, 1595, rol. 12, p. 161. "Lptox, MAINe." Birdlore, 1908, vol. 10, plate in no. 3, figs. 2, 3. Egg, Bendire, 1595, vol. 2, pl. 2, fig. 30 .

Alder swamps; nests in low bushes.

Me.-Common local summer resident. (May 15) May 1SSept. 5. Eggs, June 21. 
N. H.-Local summer resident below $2000 \mathrm{ft}$; uncommon south of White Mts. (May 11, 16) May 20-Aug. 31. Eggs, June 22-July 8.

VT.- Lncommon local summer resident. (May 17) May 21Sept. Eggs, June 13--July 30.

MAss.- Local summer resident, rare in eastern part (Essex and Middlesex Cos.), commoner in western. May 16-June 6; summer-Aug. 24. Eggs, June 15-July 3.

Coxr-L Local summer resident (Norfolk). (May 6).

248. Empidontx mismes (Baird) Baird.

\section{Least flycatcher; Chebec.}

Ridgway, Bull. 50, U. S. nat. mus., 1907, pt. 4, p. 561. Birdlore, 1908, vol. 10, plate in no. 3, fig. 1. Egg, Bendire, 1595, vol. 2, pl. 2, fig. 31 .

Tyrannula minima Baird, Proc. acad. nat. sci. Phila., 1St3, p. 2St. "Near Carlisle, Pexisrliana."

Orchards, shade trees, and borders of woods; nests in trees.

ME.-Common summer resident. (April 21) April 27-Aug. 31 (Sept. 5). Egges, June 11.

N. H.- Common summer resident below $2000 \mathrm{ft}$. April 29Sept. Egges, Mary-June 10; July 10-late July (2d brood).

YT.-Common summer resident of low country. April 29Sept.

Mass-Common summer resident. (April 22) April 2S-Sept. 21 (Oct. 2). Egges, May 20-July 1 (2 broods).

R. I.-Common summer resident. (April 22) April 27-fall. Egggs, Maỹ 2-t-June 2.

Coxx-Common summer resident. April 26-Sept. 
PASSERES.

OSCINES.

\section{ALACDIDAE.}

249. Otocoris alpestris (Linné) Bonaparte.

\section{Horned lark; Shore lark.}

Ridgway, Bull. 50, L. S. nat. mus., 1907, pt. 4, p. 303; Chapman, Bird-life, 1S9S, pl. 34. Egg. Seebohm, 1S96, p. 259. pl. 5S, fig. 15.

Alauda alpestris Linné, Srst. nat., ed. 10, 175s, rol. 1, p. 166. "IN Ainerica septentrionali."

Seashores and open fields; nests on the ground.

ME., N. H.-Common migrant and winter resident, chiefly coastwise. (Sept. 25, Milo, Me.) Oct. 1S-April (May 1-7, Monson, Me.).

TT.-Common migrant. Feb.-Mar.; (Sept. 15)-Nor.

Mass.- Common migrant, less common winter resident, mainly coastwise. (Sept. 27) Oct. 17-April 19 ( Мау 27).

R. I.-Common migrant and winter resident. (Sept. 25)April 15.

Coxx.- Common migrant and winter resident, mainly coastwise. \ок. 1S-Mar. 26.

250. OtOCORIS Alpestris PRATICOLA Henshaw.

\section{Prairie horned lark.}

Hensh., Auk, 1SSt, rol. 1, p. 264. "Ruchlaco Co., Ilt.," but tỵe locality "Dexrer, Colorado" (see Bull. 50, C. S. ma1. mus., 1907, pt. 4, p. 311, foot note). Egge, Bendire, 1595, rol. 2, pl. 5, fig. 25 .

Eromophila comuta (Wils.). Baird, Brewer, and Ridgway, Hist. No. Amer. birds, 1S7t, rol. 2, pl. 32, fig. 2 (hearl of roung).

Open fields; nests on the ground.

ME.- Lncommon migrant and summer resident; casual in winter. Feb. 10-Mar. 30; summer-`or. 26 (.Jan. 24, 1S99, Pittsfield). Egos, Mar 12-31. 
PASSERES.

OSCINES.

ALAUDIDAE.

N. H.- Uncommon migrant, and in White Mt. ralleys (? and summit of MIt. Washington, 1905) a rare summer resident. Feb. 2S-Aug. Egges and young, May 21, June 22.

V'T.- Ln'ommon migrant and lare summer resident (at Burling- ton, Commall, Mt. Mansfield, Rygate, St. Albans, Stowe). Feb.fall. Eggs, Mar.-April 19.

MAss.- Rare migrant and summer resident, occasional winter resident (Longmeadow). Feb. 19-Mar. 22; stmmer; Oct. 26Dec. 15 (winter). Eggs, July 10.

R. I.- Rale migrant: Pawtuxet, Nor. 25, 1889.

Coxx.- Uncommon migrant and summer resident (Dambury, 190S, Litchfield, 1905, Washington, 1906); "occasional in winter." Eggs, Mar. 25.

251. Otocoris Alpestris Horti Bishop.

\section{Hoyt's horned lark.}

Bishop, Auk, 1S96, vol. 13, p. 130. "CAndo, Towner Countr, North Dakota."

Open country and cousts; nests on the ground.

Coxs.- Rare migrant and (?) winter resident: Guilford, Mar. 7, 1903; West Haren, Jan. 24, 1905 (Bishop).

\section{CORVIDAE.}

252. Cranocitta CRIstata (Limué) Strickland.

\section{Blue jay; Corm-bird.}

Corvus cristrutus Limné, Ṣ̣st. nat., ed. 10, 175S, rol. 1, p. 106. "in Anerica septentrionali."

Gramulus cristatus Linné. Andubon, Birds of Amer., 1S42, vol. 4, p. 110, pl. 231. Egg, Bendire, 1895, rol. 2, pl. 5, figs. 5, 6.

Woodlands; ne'sts in trees.

ME.-Common migrant and resident. Eggs, May-June $t$. 
PASSERES.

OSCINES.

CORVIDAE.

N. H.- Common migrant and summer resident; less common in winter south of the White Mts.

VT.-Common migrant and resident. Eggs, May 11.

MAss.-Common migrant and resident. Eggs, April 2S-June 15.

R. I.- Common resident. Egges, May 2-22.

Coxx.-Common resident. Eggs, May 5-June 13.

253. Perisoreus Caxadexsis (Limné) Bonaparte.

Canada jay; Camp robber; Caribou-bird; Gray jay; Greasebird; Meat-bird; Meat hawk; Moose-bird; Venison hawk; Whiskey-Jack; Whiskey-John; Wiss-ka-chon (Indian).

Corvus canadensis Linné, Srst. nat., ed. 12, 1766, rol. 1, p. 15S, "CANADA."

Gamulus canadensis (Limné). Audubon, Birds of Amer., 1S+2, vol. 4, p. 121, pl. 234. Egg, Bendire, 1S9.5, rol. 2, pl. 3, figs. 1S, 19.

Fir and spruce forests; nests in trees.

ME.- Uncommon resident in northern part, south to Umbagog; elsemhere casual. Eggs, Mar. 16-2S.

N. H.- Uncommon resident abore $3000 \mathrm{ft}$. on Sandrich Range north; elsewhere casual.

VT.- Uncommon resident in northern part and on high peaks (MIt. Mansfield); elsewhere casual (Somerset, Aug. 5, 1S96).

MAss.- Accidental visitor: Lexington (nec Arlington Heights), Oct. 16, 18s9; Newtonville, early summer, 1875; Salem. Oct. 25, 1878 .

254. CORYus CORAx PRIXCIPALIS Ridgray.

Northern raven.

Ridgw., Manual No. Amer. birds, 1SST, p. 361. "Northery North Ayerica, froil Greenland to Alaska, south to British Columbia, Canada, \ew Brunimick." 
PASSERES.

OSCINES.

CORVIDAE.

Corvus corax Linné. Audubon, Birds of Amer., 1St3, rol. 4, p. 7s, pl. 224. Egg, Bendire, 1S95, vol. 2, pl. 4, fig. 3.

Vicinity of seacoasts and large rivers; nests on eliffs or rarely in trees.

ME.- Uncommon resident, mainly eoastwise. Eggs, April 14.

X. H.- Aecidental'visitor (in winter?).

TT. - Very rare visitor in northern part: Brandon, 1879.

MAss.-Aeeidental visitor, no recent records (Williamstown, before 1577 ).

Coxx.-Accidental visitor: South Manehester, Sept. 1S, 1890.

255. Cortus Brichrorichos C. L. Brehm.

\section{American crow.}

C. L. Brelın, Beitr. z. vögelkunde, 1S22, vol. 2, p. 56. AıERICA.

Corvus americanus Audubon, Birds of Amer., 1842, vol. 4, p. S7, pl. 225. Egg, Bendire, 1S95, vol. 2, pl. 4, fig. S-12; pl. 5, figs. $21,22$.

Ubiquitous, though avoiding dense forests in the breeding season; nests in coniferous trees.

ME.- Common resiclent, in winter chiefly confined to coast. Eggs, April 20-May 25 (June 15).

\. H.-Common migrant and summer resident; in winter less common near coast and large rivers, rare elsewhere. Eggs, April 17.

VT.-Common resident. Eggs, April 22.

MAss.-Common migrant and summer resident; common winter resident near coast, but less so inland. Eggs, April 15-June 13.

R. I.- Common migrant, summer and winter resident. Eggs, April 12-23.

Coxs.- Common migrant, summer and winter resident. Eggs, April 12-MIay 9. 
PASSERES.

OSCINES

CORVIDAE.

256. Corves ossifragus Milson.

\section{Fish crow.}

Wils., Amer. ornith., 1812, rol. 5, p. 27, pl. 37, fig. 2. "NEW Jersey. ...xear....Philadelfima." Egg, Bendire, 1895, vol. 2 , pl. 4 , figs. 16,17 .

Seaboard and near large streams; nests in trees.

TT.-Doubtfully recorded.

MAss.- Rare risitor in southern part (Cambridge, Springfield, Wareham). Mar. 16-July 16.

Cons.- Rare summer resident (Bridgeport, Fairficld, near New London, New Haven, Stratford). Mar.--Nov. 10. Egggs, May 12-16.

\section{STURNIDAE.}

257. Steries velgaris Limué.

\section{Starling.}

Linné, Syst. nat., ed. 10, 1758, vol. 1, p. 167. "Eeropa, Africa." Cones, Key to No. Amer. birds, ed. 5, 1903, rol. 1, p. 503, frontispiece. Egg, Secbohm, 1896, p. 230, pl. 54, fig. 13.

Open comtry and fields; nests in holes of buildings, trees, or cliffs.

Me.- Accidental visitor: Calais, May 4, 1859.

Mis:- Introduced at Springfield in 1897 but all disappeared; also stragghler: Agawam, April, 1908; Pittsfield, Oct. 1, 1899.

Coxx.- Local resident; apparently spreading from New lork colony (New Haren, Dec. 3, 1900; Stamford, 1900; Norwalk, 1900; Weathersfield and Stonington, 1906; Bethel, 1907; New London, 190`). Eggos, April. 
PASSERES.

OSCINES.

ICTERIDAE.

25S. 1)olichorix ortzivorts (Limé) Swainson.

Bobolink; Butter-bird; May-bird; Meadow-wink; Reed-bird; Rice-bird; Skunk blackbird.

Chapman, Handb. birds cast. No. Amer., 1895, p. 261; Auk, 189:3, vol. 10, pl. 7. Egg, Bendire, 1S95, vol. 2, pl. 6, figs. 1, 2.

Fringilla oryzivora Limné, Syst. nat., ed. 10, 175s, vol. 1, p. 179.

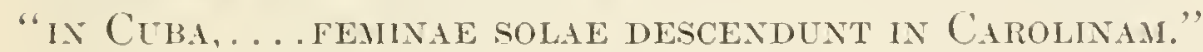

Grassy meadows; nests on the ground.

ME.-Common migrant and local summer resident. May 1Sept. 15. Eggs, Jume 6-15.

․ H.- Common migrant and summer resident at lower eleriations. May 1-Sept. 23.

TT.-Common migrant and summer resident. Eggg, June 1.

MAss.-Common migrant and summer resident. (April 27) May 1-Sept. 29. Eggs, June 1-S.

R. I.-Common migrant and summer resilent. May 10-Sept. 25. Eggs, May 30-June 3.

Coxx.-Common migrant and summer resident. May T-Sept. j. Hges, June 5 .

259. Molothrt's Ater (Boldaert) Gray.

Cowbird; Clodhopper; Cow blackbird; Cow bunting; Lazybird; Shiney-eye.

Oriolus ater Bodel., 'I'abl. d. planch. enlum. d'luist. nat., 1783, p. 37. Description in Buffon: "Amérique."

Molothrus peroris Gmel. Audubon, Birds of Aner., 1St2, vol. 4. p. 16, pl. 212. Egg, Bendire, 1895, vol. 2, pl. 6, figs. 3-4.

Open country; lays eggs in nests of other small birds.

ME.-Common migrant and summer resident. Mar. 19-Oct. 13 (Dec. 27). Eges, Мay 26-Jume 2.

N. H.- Uneommon snmmer resiclont in southem part; rare winter resident. Mar. 25-Oct. 2 (winter). Eggrs, June 1. 
PASSERES.

OSCINES.

ICTERIDAE.

IT. - Uncommon summer resident; very rare in winter. March 23-(Dec. 30).

Mass.-Common migrant and less common summer resident; occasional in winter. (Mar. 1) Mar. 11-Nor. 21; winter. Eggs, May 15-June 15 (July 1).

R. I.- Common migrant and summer resident; rare in winter. Mar. 14-Nor. 3 (Jan. 19). Eggs, May 16-June 2.

Cosx.-Common migrant and summer resident; rare winter resident. Mar.--Nor. 4; winter. Eggs, May 9-June 14.

260. Xanthocephalts xanthocephalus (Bonaparte) Jordan. Yellow-headed blackbird.

Icterus xanthocephalus Bonap., Journ. acad. nat. sci. Phila., 1S26, vol. 5, p. 223. Founded on Say: "Exgneer CantoxMENT," Iowa.

Icterns icterocephahus (Limé). Bonap., Amer. ornith., 152:5, vol. 1, p. 27, pl. 3, figs. 1, 2. Egg, Bendire, 1895, vol. 2, pl. 6, fig. $10-12$.

Open prairies, marshes, and sloughs; nests among reeds.

Me.-Aecidental visitor: Spruce Head, Metnic Id., Aug. 17, $18 S 2$.

MAss.-Accidental visitor: Eastham, two, Sept. 10, 1877; Monomoy Id., Sept. S, 1897; Watertown, Oct. 15, 1869.

Coxs.-Accidental risitor: Hartford, late July, 1Sst; New Haren, Jume, 1S7S; Stamford, July, 1888.

261. Agelaits phomxiceus (Limné) Vieillot.

Red-winged blackbird; Fire-wing; Marsh blackbird; Quonka-ree; Redwing; Swamp blackbird.

Audubon, Birds of Amer., 1842, vol. 4, p. 31, pl. 216. Egg, Bendire, 1595 , vol. 2 , pl. 6 , fig. 13-15.

Oriolus phoeniceus Limné, Srst. nat., ed. 12, 1766, vol. 1, p. 161. "IN AuERICA SEPTENTrionali." 
PASSERES.

OSCINES.

ICTERIDAE.

Open country, swamps, and marshes; nests in marsh grass or in bushes near water.

Me.-Common migrant and local summer resident. Mar. 13Oct. 13 (Nor. 9). Eggs, June 1-July 1.

N. H.- Common migrant and local summer resident in vallers; accidental in winter (Warren). Mar. 3-Nor. 11 (winter).

VT.-Common migrant and summer resident. Mar. 2-Oct.

MAss.-Common migrant and summer resident, wintering rarely in south and east. (Feb. 26) Mar. 6-Oct. 20 (Nor. 2S) (winter). Eggs, May 10-June 1S.

R. I.-Common migrant and summer resident; casual in winter. Mar. 7-Oct. 19 (Dcc. 15, Feb. 10-13). Eggs, May 12-June 16.

Cons.-Common migrant and summer resident; rare winter resident. Mar. 4-Oct. (winter). Eggs, May 30-Jume 6.

262. Agelaius phofniceus fortis Ridgway.

\section{Thick-billed redwing; Northern redwing.}

Ridgway, Proc. Washington acad. sci., 1901, vol. 3, p. 153. "Omaha, Nebraska."

Marshes and open country; nests among reeds or bushes.

Cosx.- Rare fall migrant and winter resident: North Haven, Nov. 11, 1903, Dec. 25, 1901, Jan. 10, 1902 (Bishop).

263. Sturnella iagia (Limné) Swainson.

\section{Meadowlark; Marsh quail; Old-field lark.}

Chapman, Handb. birds cast. No. Amer., 1895, p. 265; Birdlife, 1898, pl. 39. Egg, Bendire, 1595, vol. 2, pl. 6, figs. 20, 21.

Alauda magna Linné, Syrst. nat., ed. 10, 175s, rol. 1, p. 167. "in Anierica, Africa."

Open fields and marshes; nests on the ground.

ME.- Rare migrant and local summer resident in southern 
PASSERES.

OSCINES.

ICTERIDAE.

counties (Rangeley, April 21, 1897); rare in winter (Calais, Jan., 1899; Cape Elizabeth; Westbrook, Jan. 16, 18, 1904). Mar. 15Nor. 19 (winter). Eggs, June-(July 22).

․ H.-- Lncommon summer resident north to White Mt. ralleys; rare winter resident on coast. Mar. 26-Oct. 12 (winter).

T'T.-Uncommon local summer resident; rare winter resident (L. Champlain, Middlebury, Pawlet, St. Albans, West Rutland). April 2-Nor. 1.5 (winter).

MLAss.-Common summer resirlent, and near the coast an uncommon winter resident. Mar. 2-Nor.; winter. Eggs, May 11June 28.

R. I.-Common summer resident, and in coastal marshes a common winter resident. Eggs, May 6-30.

Coxx.-Conmon summer resident and less common winter resident coastwise. Eggs, June 6.

264. ICterus spurius (Linné) Bonaparte.

\section{Orchard oriole.}

Chapman, Handb. birds east. No. Amer., 159.5, p. 266; Birdlife, 1898, pl. 36. Egg, Bendire, 1895, vol. 2, pl. 7, fig. 3-5.

Oriolus spurius Linné, Srrst. nat., ed. 12, 1766, vol. 1, p. 162. "IN Alierica SEPTENTrionali."

Shade trees and orchards; nests pensile in trees.

Me.-Accidental risitor: near Auburn; near Calais; Thomaston.

N. H.-Acridental visitor: Rollinsford.

Vт.- Very rare summer resident: Bemnington; Brattleboro (with young); Middlebury, June 1, 1883.

Vhass. - Ritre local summer resident near coast (to Ipswich) and in western valleys. (May 1) May S-Aug. 15. Eggs, May 2SJune 25.

R. I.- Rare summer resident. May 14-July. Eggs, May 30June 3. 
PASSERES.

ICTERIDAE.

Coxx-C Common summer resident. Mar t-Aug. Eggogs, June 11-14 (Aug.).

265. ICterc's Gadizla (Linné) Cones.

Baltimore oriole; Fire-bird; Golden oriole; Golden robin; Hang-bird; Hang-nest; Pea-bird.

Coracias galbula Imné, Syst. nat., ed. 10, 175s, rol. 1, p. 10 s. "IN AMERICA."

Icterus baltimore (Limné). Audubon, Birds of Amer., 1842, rol. 4, p. 37, pl. 217. Egge, Bendire, 1595, vol. 2, pl. 7, fig. 6-9.

Orchirds and shade trees; nest pensile in trees.

ME.-Common summer resident mainly in southern counties. May 9-Aug. 30 (Sept. 10).

‥ H.-Common summer resident at lower elevations. May 6-Sept. 6 (Sept. 20).

VT.-Common summer resident; accidental in winter. May 10-Sept. (Dec. t, 1SS4).

MAss.-Common summer resident; accidental in winter. (April 29) Mar 1-Sept. 9 (Nor. 15, 1885, E. Templeton; Nor. 18, 1Ss6, Hyde Park). Eggos, May 2t-July 4.

R. I.-Common summer resident. May 4-Sept. 1. Egags, May 2t-2S.

Coxx.-Common summer resident. Mar 1-early Sept. (Nor. 15, 185.5, Portland). Eggs, June 6.

266. ICterus Buldocki (Swainson) Bonaparte.

\section{Bullock's oriole or troupial.}

Audubon, Birels of Amer., 18t2, vol. 4, p. 43, pl. 21S. Egrg, Bendire, 1895, vol. 2, pl. 7, fig. 10-13.

Tanthormus bullockii Swains., Phil. natg., 18:- , new ser., wol. 1, p. 436. "Table rand ... Mex Mexico."

Open country along streams; nests in trees.

ME.- Arerdental risitor: Sorrento, about middle of Nor., 1 Ss. . 
PASSERES.

OSCINES.

ICTERIDAE.

267. Euphagus carolinus (Müller) Richmond.

\section{Rusty blackbird; Rusty grackle.}

Turdus cerolinus Müll., Linné's Vollstand. natursyst., suppl., 1766, p. 140. "Carolina."

Quiscalus fermgineus Linné. Audubon, Birds of Amer., 1S42, vol. 4, p. 65, pl. 222. Egg, Bendire, 1995, vol. 2, pl. 7, fig. 14-16.

Swamps, marshes, and wet woods; nests in bushes and low trees.

Ne.-Common migrant and in northern part a rare summer resident; aceidental winter resident (Calais, 1S99). Mar. 17May; summer-Oct. 29 (winter).

N. H.- Common migrant and in northeast part, a rare summer resident (Mlegalloway R.). Mar. S-May; summer; Sept. 9Oct. 16 .

$V_{\mathrm{T}}$.- Common migrant.

Miss-Common migrant, atcidental in winter (Brookline, Feb. S, 1879; Coneord, Jan. 22, 1905; Fresh Pond, Feb. 20, 18S7; Jamaiea Plain, winter 1907-S). Mar. 7-May 18 (May 30); Sept. 12-Nov. 27 (winter).

R. I.-Common migrant. Mar. 13-(? June 5); Sept.-Oet. 23.

Cons.-Common migrant, and rare winter resident. Mar.April 19; Sept. 11-Xov. (Jan. 16, 29, Feb. 6, Milford; Dec. 25, 1908, New Haven).

268. Quiscalus quiscula (Limé) Jordan.

\section{Purple grackle; Crow blackbird.}

Chapman, Handb. birds east. No. Amer., 1595, p. 269. Eggr, Bendire, 1895, vol. 2, pl. 7, fig. 21-23.

Gracula quiscula Limné, Syst. nat., exl. 10, 1758, vol. 1, p. 109. "1N America septentrionali."

Quiscalns versicolor Vieill. Audubon, Birds of Amer., 1St2, vol. t, p. 5S, pl. 221. 
PASSERES.

OSCINES

ICTERIDAE.

Open country, groves, ficlds; nests in branches of coniferous trees, ranely in cavities or bushes.

․ H.- Acriclental risitor: 'Tilton, Sept. 13, 1902.

VT. - Smmmer resident (Perkins).

MAss. - Occasional trpical birds breed in southermmost part and coastwise to Cambridge.

R. I.-Common smmmer resident in southern part, where most birds are ypical; casual in winter. Mar. 6-Sept. 21 (Nor., Dec. 31). Eggs, April 25-June 6.

Coxx.- Common summer and accidental winter resident. (Feb. 13) Mar. 1-Nor. (winter, East Haven).

269. Quiscalus Quiscula AEneus (Ridgway) Stejneger.

\section{Bronzed grackle; Crow blackbird.}

Quiscalus aeneus Ridgr., Proc. acad. nat. sci. Phila., 1869, p. 134. "Mississippl region of U. S., EAst to Alleghani MTs." etc. Egg, Bendire, 1595, rol. 2, pl. 7, figs. 26, 27.

Open country, frequenting clumps of coniferous trees in breeding season; nests in branches of trees, or less often in cavities or bushes.

\E.-Common migrant and local summer resident. Mar. 19Oct. 26. Egggs, Мay 27-Jume 21.

X. H.- Uncommon local smmmer resident; casual in winter. Mar. 12-Nor. (Dec. 24; 1907, Lancaster; Jan.).

VT.- Lneommon local summer resident. April 11-Oct.

Mass. - Common migrant and local summer resident; rare in winter near coist (also Springfield). (Feb. 23, 1909, Boston) Marr. 10-Nor. 22 (winter). Eggs, May 2-June 1.

R. I.- Common migrant and in the northern part a less common summer resident, interglading with Q. quiscula. Mar. 5- †ov. 1. Eggs, Мay 1-17.

Coxx.-Common migrant. Mar. 5-April; Oet.-Nov. 
PASSERES.

OSCINES

FRINGILLIDAE.

270. Hesperiphona respertina (IV. Cooper) Bonaparte.

Evening grosbeak; Sugar-bird.

Fringilla respertina Coop., Ann. lye. nat. hist. New York, 1825, vol. 1, pt. 2, p. 220. "SAlit Ste. Marie, Near Lake Superior."

Coccothraustes respertimus (Coop.). Chapman, Handb. hirds east. No. Amer., 1595, p. 279; Audubon, Birds of Amr., 1S+1, vol. 3, p. 217, pl. 207.

Tooded districts; nests in trees.

ME.- Accidental risitor: several records in winter of 18\$9-90, Jan. 10-Mal. 18; Toodfords, Mar. 6, 1909.

X. H. - Accidental visitor: several records in winter of 1859-00, Jan. 6-\ау 1.

VT.-Accidental visitor: Burlington, about Feb. 20, 1890; Rutland, Feb. 1909.

MAss - Accidental risitor, first recorded in the 1890 flight (.Ian. 1-April); also Wellflect, one, Dac. 5, 1903; Beverly, flock of five, Mar. 23. 1904; So. Sudbury, Feb. 14, 1909; 'Townsend, Feb. 7, 1909; Williamstown, Jan. 26, 1909.

Coxx.- Accidental visitor, first recorded in 1890 flight (Fast Hampton, Mar. 2; Carylordsville, Mar. 10; Portland, Feb., Mar.); also Litchfield, ? Feb. 1, 1905, and Feb. 11, 1905; Bristol, Feb. 27, 1907.

271. Pixicola exucleator leucura (Mïller) Richmond.

Pine grosbeak; Canadian pine grosbeak; Mope.

Loxia lencura Müller, Linnés Vollstand. natursyst., suppl., 1776, p. 150. "CANADA."

Pinicola cmuclcator canadensis (Cab).). Ridgwar, Bull. 50, L'. S. nat. mus., 1901, pt. 1, p. 60.

Pyrrhula emucleator Temm. Audubon, Ornith. biogr., 1838, vol. 4, p. 414, pl. 3iss. Egg. Capen, 1\$\$6, pl. 7, fig. 13. 
PASSERES.

OSCINES.

FRINGILLIDAE.

Spruce and fir forests; mests in trees.

ME. - Fall and winter visitor of rarying abundance, and in northern forests a rare resident. Oct. 25-April 18; summer. Eggs, May $11-27$.

N. H. - Fall and winter visitor of varying abundance, and uncommon summer resident above $3000 \mathrm{ft}$. in White Mts., north. Oct. 1S-April 20; summer.

VT.- Winter visitor of varying abundance. Nov. 15-Mar. 24.

MAss. - Irregular winter visitor of rarying abundance. Oct. 24-April 4 (April 24).

R. I. - Irregular winter visitor, sometimes common.

Cosx. - Irregular winter visitor, sometimes common. Nov. 24Mar. 31.

272. Carpodacus purpureus (Gmelin) Gray.

\section{Purple finch; Red linnet.}

Fringilla purpurea Gmelin, Sỵst. nat., 17SS, vol. 1, pt. 2, p. 923. "CArolina."

Erythrospiza purpurea (Gmel.). Audubon, Birds of Amer., 1841, vol. 3, p. 170, pl. 196 Egg, Capen, 1S86, pl. 7, figs. 14, 15.

Orchards, shade trees, and forests; rests in trees.

ME.-Common migrant and summer resident; rare winter resident (Rangeley Lakes, Jan. 15, 1S84). Mar. 21-Oct. (minter). Eggs, June 4.

N. H.-Common summer resident below $3000 \mathrm{ft}$; rare in winter. April 15-Dec. 22; winter.

VT.-Common summer resident.

Mass.- Common migrant, less common summer, and occasional winter resident. Mar. S-Oct. 21; winter. Eggs, May 25-June 19 (! Sept. 10).

R. I.- Common summer and uncommon winter resident. April 7-Oct.; winter. Egges, May. 19-June S. 
PASSERES.

OSCINES.

FRINGILLIDAE.

Coxs.-Common summer and uncommon winter resident. Eggs, June; July 22 (2d brood).

273. Loxia cLrvirostra amor (Brehm) Ridgway.

American crossbill; Red crossbill; Screwbill.

Ridgway, Bull. 50, U. S. nat. mus., 1901, pt. 1, p. 47.

Crucirostra minor Brehm, Naumannia, 1S.5., p. 193. “DER Nordalerikanischen Freistatex."

Loxia cumirostrata Linné. Audubon, Ornith. biogr., 1835, rol. 2, p. 559, p]. 197. Egg, Capen, 1SS6, pl. S, figs. 1, 2.

Coniferous forests; nests in trees.

Me., N. H.- Resident, nomadic and irregularly abundant. Nests from Jan.-Oct.

VT.- Irregular at all seasons, but commonest in winter; breeds occasionally. Eggss, Feb., Mar.

MAss. - Irregularly common winter visitor, and occasional at all seasons; nests rarely (Framingham, 1S84; Highland Light, April, 1889; Tyngsborough, Aug., 1875).

R. I.- Irregularly common winter visitor, and oecasional at other seasons; not known to breed.

Coxv.- Irregular winter visitor, sometimes common. Oet. 7 -spring.

274. Loxia lectoptera Gmelin.

\section{White-winged crossbill.}

Gmel., Srst. nat., 17SS, rol. 1, pt. 2, p. S4t. "IN SINC Hudsonis \& Noveboraco." Audubon, Birds of Amer., 1St1, vol. 3 p. 190, pl. 201.

Coniferous forests; nests in trees.

ME.- Uncommon resident of northern part; elsewhere an irregular risitor. Nest, June. 
PASSERES

FRINGILLIDAE.

N. H.- Uncommon resident above $3000 \mathrm{ft}$. in White Mts. and north; elsewhere an irregnlar visitor.

VT.- Irregnlarly common winter visitor, rare in summer in northern part and on higher peaks. Nested at Lunenburg, 1 S7S.

MAss. - Irregular fall and winter visitor, sometimes common. Oct. 22-15ay 12 (May 25); (June 4, 1900, Lanesboro; June 13, 1866, Newtonville).

R. I.- Rare and irregular winter risitor. Jan. 14-Feb. 24.

Coxx.- Rare and irregular winter visitor. Nor. 6-1Lar. 7.

275. Acanthis horvemaxim Exilipes (Cones) Stejneger.

\section{Hoary redpoll.}

Aegiothns exilipes Coues, Proc. acad. nat. sci. Phila., 1561, p. 385. "Fort Sinipson."

Fringilla borealis Temm. Andnbon, Ornith. biogr., 1839, vol. 5, p. 87, pl. 400.

Aretie thickets in summer, open tree growth in winter migrations; nests in low trees.

ML.- Rare winter visitor: Westbrook, Jan. 26, 1896; eastern Maine (Boardman).

N. H.- Rare winter visitor: Hampton.

YT.-Doubtfully reported.

Mass. - Rare winter visitor (some ten instances). Nov. 15Mar. 20.

276. ACanthis linaria (Limné) Bonaparte and Schlegel.

\section{Redpoll; Lesser redpoll; Mealy redpoll.}

Ridgway, Bull. 50, U. S. nat. mus., 1901, pt. 1, p. S5. Egg, Seebohm, 1896, p. 246, pl. 57, figs. 6, 7 .

Fringilla linaria Linné, Syst. nat., ed. 10, 175\$, vol. 1, p. 182. "n Evropa." Audubon, Ornith. biogr., 1838, vol. 4, p. 523, pl. 375 . 
PASSERES.

OSCINES.

FRINGILLIDAE.

Aretie thickets in summer, open tree growth in winter migrations; nests in low trees.

Me.- Winter visitor of irregular abundance, and rare summer resident (Calais). Oet. 12-April 27 (May 19, 1875; summer).

N. H. - Winter visitor of irregular abundance. Nov. 1-April.

VT.- Winter visitor of irregular abundance; (nest and three eggs said to have been found in late March, 187S, at Taftsville). Nov. 11-May 1 (May 11).

Mass.- Irregularly eommon fall and winter visitor. (Oct. 16) Oct. 25-April 25 .

R. I.- Irregularly common fall and winter visitor. Oet.-Mar. 25.

Conn.- Irregularly common fall and winter visitor. Fail-Mar. 31.

277. Acanthis livaria holboellil (C. L. Brehm) Dubois.

\section{Holboell's redpoll.}

Ridgway, Bull, 50, U. S. nat. mus., 1901, pt. 1, pp. \$0, 99.

Linaria holboellii C. L. Brehm, Handb. d. naturgesch. aller vögel Deutschl., 1S31, p. 280. "IN Das mittlere Deutschland."

Acanthis holboellii Bonaparte and Schlegel, Monogr. Loxiens, 1850 , p. 50 , pl. 53.

Aretic thickets in summer, open tree growth in winter migrations; nests in low trees.

Me.- Rare winter visitor: Gorham, Feb. 3, 1903; North Bridgton, Nov. 25, $187 \mathrm{~S}$.

Mass.- Rare winter visitor: Lexington, Mar. 10, 1890; Swampseott, two, Mar. 26, 1SS3.

27S. Acanthis linaria rostrata (Cones) Stejneger.

\section{Greater redpoll.}

Ridgway, Bull. 50, U. S. nat. mus., 1901, pt. 1, pp. \$0, 91. 
PASSERES.

OSCINES.

FRINGILIIDAF.

Aegiothus rostratus Cones, Proc. acad. nat. sci. Phila., 1S61, p. 378. "Jacobsimar, Greentanjo."

Limota homemami Holb. Dresser, Birds of Europe, 1871-\$1, vol. 4, pl. [190], lower fig:

Aretic thickets in summer, open tree growtl in winter migrations; nests in low trees.

ME.- Winter visitor of irregular abundance. Dec. 12-Mar. 15.

X. H.- Unsatisfactorily recorded.

MLss.- Winter visitor near coast, irregular and sometimes common. Dec. 27-Feb. 25.

R. I.- Rare winter visitor: East Provilence, Mar. 14, 1896.

Conv.- Rare winter visitor: New Haven, Dec. 17, 1 S7S.

279. Astragalinus tristis (Limmé) Cabanis.

American goldfinch; Lettuce-bird; Thistle-bird; Wild canary; Yellow-bird.

Fringilla tristis Limné, Syst. nat., ed. 10, 175s, rol. 1, p. 1 S1. "IN AMERICA sEPTENTRIONALI."

Spimus tristis (Linné). Chapman, Handb. birds east. No. Amer., 1895, p. 286; Birl-life, 1898, pl. 52. Egg, Capen, 18s6, pl. S, fig. 3.

Orchards, shinde trees, and edges of woods in summer, weedy fields and open country in winter; nests in trees.

Mss-Common migrant and summer resident; uncommon in winter. liggrs, July 29-Aug. 18 (Sept. 5).

N. H.-Common migrant and summer resident; uncommon in winter from White Mts. soutl.

YT.-Conmon migrant and summer resident, less common in winter. Eggs, July 26.

MAss. - Common migrant and summer resident, less common winter resident. Eggs, July 21-Aug. 11.

R. I.-Common summer, and less common winter resident. Egggs, July 1-Aug. 7 .

Coxx-C Common resident. Egggs, July-Aug. 
PASSERES.

OSCINES.

FRINGILLIDAE.

280. Spinus pixts (Wilson) Stejneger.

Pine siskin; Gray linnet; Pine finch; Pine linnet.

Fringilla pimus Wils., Amer. ornith., 1810, vol. 2, p. 133, pl. 17, fig. 1. "Neighbourhood of Philadelphia."

Linaria pinus (Wils.). Audubon, Birds of Amer., 1S41, vol. 3, p. 125, pl. 1S0. Egg, Capen, 1S56, pl. \&, fig. 4.

Coniferous forests in summer, open country and coniferons trees in fall and winter; nests in trees.

ME.-Common resident of northern part; elsewhere of irregular occurrence mainly in fall and winter. Eggs, April.

N. H.-Common resident, breeding mainly above $3000 \mathrm{ft}$. in White Mts. Eggs, mid-April.

YT.-Common winter risitor, less common in summer, breeding irregularly. Nesting, April-May 15.

MAss.- Irregularly common fall and winter visitor, sometimes lingering till late spring and rarely nesting. Sept. 19-Xay 30 (June S, July 15, summer). Eggs, May 9-29.

R. I.-- Irregularly common fall and winter risitor. Oct.-April 17 (Mar 31).

Coxx.- Irregularly common fall and winter risitor. Oct.April (May 20).

281. Spints spints (Linné) Stejneger.

\section{European siskin.}

Fringilla spinus Linné, Srst. nat., ed. 10, 175s, rol. 1, p. 1 s1. "in Europae juxiperetis."

Chrysomitris spinus (Limné). Dresser, Birds of Europe, 187181, rol. 3, p. [541], pl. [169]. Egg, Secbohm, 1896, p. 242, pl. 56, fig. 1 .

Coniferons woods; nests in trees.

MAss.-Cambridge, Ang. 11, 13, 17, 1904, an apparently wild bird (Brewster). 
2S2. Plectrophexax xiralis (Linné) Stejneger.

\section{Snowflake; Snow bunting; White snowbird.}

Emberiza nivalis Linné, Sỵst. nat., ed. 10, 175S, rol. 1, p. 176. "in alpible Lappoxiae, Spitzbergate ad sinum Hudosonis ALIBIQLE."

Plectropleanes miralis (Linné). Audubon, Birds of Amer., 1S41, rol. 3, p. 5.5, pl. 15.5. Egg, Seebohm, 1896, p. 247, pl. 57, figs. 9, 10.

Open fields, coasts, and barrens; nests on the ground.

ME.-Common migrant and winter resident (? accidental in summer, flock, Mt. Ktaadn, early Aug. 1\$69, fide Minot). (Oct. 10) Oct. 1.5 -April 13.

N. H.-Common migrant and winter resident; accidental in summer (Webster, June, a cripple). Oct. 1S-April 6.

VT.-Common migrant and winter resident. Nor. 17-spring.

Mass - Common winter resident, especially coastwise. Oct. 12-April 3 (Mar).

R. I.-Common winter resident. Oct. 29-Mins. 29.

Coxx- Irregularly common winter resident. Nor. 1t-Mar.

2S3. Calcarits lappoxicus (Limné) Stejneger.

\section{Lapland longspur.}

Ridgway, Bull. 50, L. S. nat. mus., 1901, pt. 1, p. 1.5.5.

Fringilla lapponira Linné, Srst. nat., ed. 10, 175s, vol. 1, p. 180. "Ix Lappoxia."

Plectrophanes lapponicus (Linné). Dresser, Bircls of Europe, 1S71-S1, vol. 4, pl. [22:3]. Egg, Seebohm, 1S96, p. 24S. pl. 57, figs. 11, 12.

Open barrens and plains; nests on the ground.

Me.- Rare migrant. Mar. 10-16; Oct. 26-Dec. 29.

N. H.- Rare migrant in late fall: Hampton Beach, Nor. 30, 1 S99. 
PASSERES.

OSCINES

FRINGILLIDAE.

VT.- Rare or aecidental migrant: Brandon, Feb. 21, 1879; ?'Tormshend.

MAss.- Rare spring and uncommon fall migrant and winter resident mainly coastwise. Feb. 22-May 1; Oct. 9-Dec. 11; winter.

R. I.- Uncommon migrant and rare winter resident. (Jan. 6, Feb. 16).

Coxs.- Uncommon migrant; may winter. (Jan. 26, 1853, Portland) Feb. 11-Mar. 16; Nor.

284. Calcarius orvatus (Townsend) Stejneger.

\section{Chestnut-collared longspur.}

Plectrophanes ornata Towns., Journ. acad. nat. sci. Pliila., 1837, vol. 7, pt. 2, p. 1S9. " "Prairies of the Platte River." Audubon, Birds of Amer., 1841, vol. 3, p. 53, pl. 154.

Open plains; nests on the ground.

Me.-Accidental risitor: Searborough, Aug. 13, 1856.

Mass.-Accidental risitor: Magnolia, July 28, IS76.

28.5. Pooecetes girduneus (Gmelin) Baird.

Vesper-sparrow; Bay-winged bunting; Grass finch; Gray-bird; Ground sparrow; White-tailed field sparrow.

Fringilla graminea Gmel., Syst. nat., 178s, rol. 1, pt. 2, p. 922. "in Noveboraco."

Emberiza graminea (Gmel.). Audubon, Birds of Amer., 1St1, vol. 3, p. 65, pl. 159. Egg, Capen, 18\$6, pl. S, fig. 9-12.

Grassy fields and uplands; nests on the ground.

Me.-Common migrant and summer resident. (Mar. 30) April 9-Oct. 28. Eggs, late May-June 24; late July-Aug. (2d brood).

N. H.- Common migrant and summer resident at lower elevations. Mar. 29-Oct. (Nor. 14). 
PASSERES.

OSCINES.

FRINGILLIDAE.

VT.-Common migrant and summer resident. Mar. 31-fall. Eggs, June 6.

Mass - Common migrant and summer resident. (Mar. 17) Mar. 27-Oct. 24 (Nor. 10; Dee. 25, 1889, Somerville). Eggs, Nay 10-June 10.

R. 1.-Common migrant and summer resident. Mar. 19Oct. 17. Eggs, Nay 5-21.

Coxs.-Common migrant and summer resident; rare winter resident. Late Mar.-Nor. (winter). Eggs, May 22-July 5.

2S6. Passerculus princers Maynard.

\section{Ipswich sparrow; Gray-bird.}

Mayn., Amer. nat., 1872, vol. 6, p. 637. “Iеswich," Mass.

Ammodramus princeps (Mayn.). Dwight, Mem. Nuttall ornitl. club, 1895, no. 2, p. 21, plate.

Sand dunes and sea shores; nests on the ground (Sable Id., N.S.).

Me.- Rare migrant coastwise; may winter rarely. Mar. 20April S; Oct. 11-Nov. 17 (Jan. 23, 1886, near Pine Point).

N. H.- Rare migrant coastwise. Mar.-April; late Oct.-early Dec. (nec Lake Umbagog.)

MAss.-Common migrant and rare winter resident among coastal dunes; casual inland (Fresh Pond, Cambridge). Oct. 12April 12.

R. I.- Lncommon migrant and winter resident among coastal dunes. Oct. 20-April 11.

Coxs.- Lncommon migrant and winter resident coastwise. Oct.-Mar. 24 .

287. Passenculds sandwichensis savacka (Milson) Ridgway.

\section{Savanna sparrow.}

Ridgway, Bull. 50, L. S. nat. mus., 1901, pt. 1, p. 192.

Fringilla sarmena Wils., Amer. ornith., 1811, vol. 3, p. 55, pl.

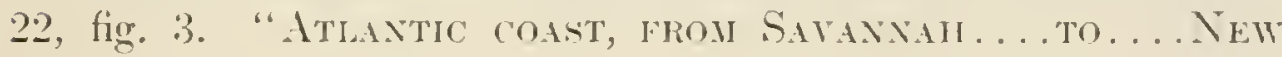


PASSERES.

OSCINES.

FRINGILLIDAE.

York." Audubon, Ornith. biogr., 1839, vol. 5, p. 516, pl. 109. Egg, Capen, 1\$\$6, pl. S, fig. 5-S.

Grassy fields and marshes; nests on the ground.

ME. - Common summer resident, acuidental in winter. (Jam. 24, 1S97, Seguim Id.) April 6-early Nor. (Nor. 2S). Egggs, May 31-June 1s; July (2d brood).

X. H.-Common summer resident at lower elevations, uncommon on barren summits of White Mts. (Mar. 31) April 15Oct. 27. Eggs, June 16-lite July.

YT.-Common summer resident. April T-fall.

MAss.-Common miglant and summer resident; lan winter" resident near coast (Ipswich, Longmeadow, Sandwich). (Mar. 22) April 5- \or. 12 (winter). Eggs, May 21-June 20.

R. I.- Common migrant, less common summer and rare winter resident. April 4-Oct. 23 (winter). Eggs, May 17-29.

Coxs.-Common migrant and summer resident. April-Nor.

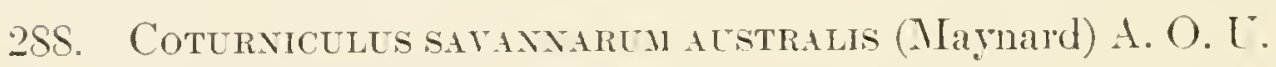
comm.

\section{Grasshopper sparrow; Yellow-winged sparrow.}

Ammodromus anstratis Mayn., Amer. exch. and mart, 1SST, vol. 3, p. 33. "BAHAMAs . . F Forid.."

Ammodramus savamnarum passerinus (Tils.). Chapman, Handb. birds cast. No. Amer., 1895, p. 293.

Emberiza passcrina (Wils.). Audubon, Birds of Amer., 1841, vol. 3, pl. 162. Egg, Capen, 1S\$6, pl. \&, fig. 13-15.

Grassy fields and meadows; nests on the ground.

ME.- Rare summer visitor (? or resident) east to Calais. May 24-June 29.

X. H. - Rare summer resident in lowlands south of Lake Winnepesauliee. May 13-?

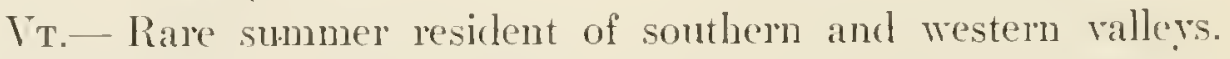
Eggs, July 17. 
FRINGILLIDAE.

Mass. - Uncommon local summer resident near coast and in western rallers. (April 25) May 10-Sept. 1 (Dece 10, 1892, Arlington).

R. I.-Common local summer resident. April 22-()et. 23. Eggs, May 30-June 5.

Coxs.-Common summer resident in southern part and Connecticut valley. Late April-?

289. Amodrames hexsiown (Aulubon) Gray.

\section{Henslow's sparrow.}

Emberiza henslowii Aud., Omith. biogr., 1\$31, vol. 1, p. 360, pl. 77. "opposite Cincixati, ñ the state of Kextucky."

Ammodramns henslowii (Aud.). Chapman, Handb. birds east. No. Amer., 189.5, p. 294. Egg, Capen, 1Ss6, p]. 9, figs. 1, 2.

Meadows and sedgy weed-grown bogs; nests on the ground.

N. H.- Rare local summer resident north to White Mt. vallers (Wonalaneet). April 17-Aug.

$V_{\text {T. }}$ - Rare local summer resident in southern part (Pownall).

Mass. - Uncommon local summer resident. May 6-Sept. (Nov. 6, Osterville). Eggs, May 25.

R. I.- Rare migrant and summer resident: Cranston, late April, 1S7t; Warwick, Oct. 6, 1S9S; Westerly, June 6 and S, 1900.

Coxx-Rare local summer resident (Fastford, Killingworth, Woodstock, etc.). May-fall. Eggs, -Aug. 6.

290. Amodranes catodicetes (Gmelin) Swainson.

\section{Sharp-tailed sparrow.}

Chapman, Iandh. birls cast. No. Amer., 1895, p. 295; Audubon, Bircls of Amer., 1St1, vol. 3, p. 10S, pl. 17t. Egg, Capen, 1886, pl. 9, figs. 3,4 .

Oriolus caudacutus Gmel., Sỵst. nat., 17s , vol. 1, pt. 1, p. 394. "IN Noremoraco." 
PASSERES.

OSCINES.

FRINGILLIDAE.

Salt marshes; nests on the ground.

ME.- Rare summer resident locally in Cumberland Co. June 5-late Oct. (? Noy.).

X. H.- Rare summer resident of the coastal salt marshes. Spring-Oct. 14.

V'T.- Doubtfully rerorded (Lumenburg).

MAss. - Uncommon summer resident of the coastal salt marshes (Ipswich); rare winter resident (West Barnstable). May 19-Nor. $S$ (winter). Eggs, Jume S-July 12.

R. I.- Common summer resident of the coastal salt marshes. May 15-Oct. 2. Egges, May 24-July 14.

Coxs.-Common summer and rare winter resident of the coastal salt marshes. April 1t-Xov. (winter). Eggs, May 31June 9 .

291. Ammodrames velsoni (Allen) Norton.

\section{Nelson's sparrow; Nelson's sharp-tailed finch.}

Ammodromus caudacutus var. nelsoni Allen, Proc. Boston soc. nat. hist., 1875, vol. 17, p. 293. "CAluziet Marshes xear Chicago, Ill.."

Immodramus caudacutus nelsoni Alken. Dright, Auk, 1896, vol. 13, p. $273-274$, pl. 4, lower fig.

Iarshes; nests on the ground.

Me.- Uncommon migrant. May 22-June 5; Oct. 9-25.

IAss. - Lncommon fall migrant; rare winter resident on south coast. Sept. 25-Oct. 15 (winter).

Coxs- - Rale spring and uncommon fall migrant on coastal marshes. May; Oct.

292. Awiodrants Nelsoxi slbvirgates (I)wight) Norton.

Acadian sharp-tailed sparrow.

4 mmodramus coudacutus subrirgatus 1) wight, Auk, 1Ss7, vol. 4 
p. 233. "Hinlsborough, Albert Co., New Brexswick." Dwight, Auk, 1896, vol. 13, pl. 4, upper fig.

Brackish or freshwater marshes; nests on the ground.

Me.- Uncommon migrant and local summer resident coastwise from Sagadahoc Co., north. May 20-summer; Sept. 10-Oct. 24 (Nor. 15).

MIAss. - Uncommon migrant coastwise (once at Springfield). May 20-June 11; Sept. 3-Nor. 5.

R. I.- Uncommon migrant: Charlestown Beach, four, Oct. 15, 1899; Point Judith, April 27, 1SS7.

Cosx.- Rare spring and uneommon fall migrant. Late MayJune 9; Oct.

293. Ammodramus maritmes (Wilson) Swainson.

\section{Seaside sparrow or finch; Meadow chippy.}

Audubon, Birds of Amer., 18t1, vol. 3, p. 103, pl. 172. Egg, Capen, 1886 , pl. 9, figs. 5,6 .

Fringilla maritima Wils., Amer. ornith., 1S11, vol. 4, p. 6s, pl. 34, fig. 2. "Low, rush-COVERED sea islaxds aloNg our ATLANTIC COAST."

Salt marshes; nests on the ground.

Me.-Aceidental visitor: Shark Id., Aug. 18, 1884.

N. H.- Erroneously recorded for A. caudacutus.

MIASS. - Rare local summer (Westport) and winter resident on south coast. April 14-(winter). Eggs, July 17.

R. I.- Lncommon summer resident at Point Judith and Middleown marshes. Eggs, July 14.

Coxs.-Common summer and possibly rare winter resident of the salt marshes. Late May-Sept. (Dec. 9, 1S89, Bridgeport). Eggs, June S-19. 
PASSERES.

OSCINES.

FRINGILLIDAE.

294. Choxdestes gramuces (Say) Bonaparte.

\section{Lark sparrow; Quailhead; Road-bird.}

Fringilla grammaca Say, Long's Exped. to Rocky Mts., 1S23, vol. 1, p. 139. "Belle FONTAN, As WELL AS AT SEVERAL OTHER poINTs on this [MIssourl] RIVER." Bonap., Amer. olnith., 1825, vol. 1, p. 47, pl. 5, fig. 3 .

Grassy fields; nests on the ground or in low trees and bushes.

Mass.-Rare visitor (seven records). April 29; Aug. 12-Nor. 25.

295. ZoNotrichia LeCCOPHRT's (Forster) Swainson.

\section{White-crowned sparrow.}

Emberiza leucophrys Forst., Phil. trans. roy. soc. London, 1772, rol. 62, p. 426. "IN America Boreali ad Sinum Hudsonis."

Fringilla lencophrys Gmel. Audubon, Birds of Amer., 1841, vol. 3, p. 157 , pl. 192.

Edges of thickets; nests on the ground or in low bushes.

Me.-Uncommon migrant; casual in summer (Androscoggin Lake, June, 1S9S; Farmington, late June, 18S7; Woolastook Valley, Aug.). (May 2) May 5-25; (June, Aug.); Sept. 22-Oct. 22 (Nor. S).

N. H.- Uncommon migrant. May 6-25; Sept. 21-Oct. 11.

TT.- Uncommon migrant; said to have bred at Rutland; nest built and one eggr laid at East Berkshire. May 14 (July, 1906, Mr. Mansfield); Oct. $4-24$.

Mass.- Uncommon migrant. May 4-27 (June 6) ; Sept. 23Nov. 5.

R. I.- Lncommon migrant; casual in summer (near Providence, July 9). May; Oct.

Coxs.- Uncommon migrant; may winter rarely. (Mar. 20) May 10-15; Oct. 12-Dec. 5. 
FRINGILLIDAE.

296. Zoxotrichia Albicolus (Gmelin) Swainson.

White-throated sparrow; Mountain lark; Peabody-bird.

Fingilla albicollis Gmel., Syst. nat., 17SS, rol. 1, pt. 2, 1). 921. "is Pexsicinata."

Fringilla pemsylcanica Latham. Audubon, Birds of Amer., 1St1, vol. 3, p. 153, pl. 191. Egg, Capen, 1S86, pl. 10, figs. 5, 6.

'Thickets; nests on the ground, rarely in bushes.

Me.-Common migrant, and from Cumberkand Co. north, a common summer resident; accidental in winter (Falmouth, winter, 1904; Lewiston, Mar. 30, 1904; Lubec, Mar. 24, 1906; Saco Jan. 20, 1SS6). April 1S-Nov. 9 (Nov. 20) (winter). Eggs, May 24-June 13.

N. H.-Common migrant, and a common summer resident to $5000 \mathrm{ft}$. in northern half, local in southem. April 23-May 14; summer; Sept.-Oet.

VT.-Common migrant, and except at lower elevations, a less common summer resident; accidental in winter (Brattleboro, Dec. 25, 1904, Mar. 31, 1907). April 29-Oct. 24 (winter). Eggs, June 21-July 3.

MAss. - Common migrant and at higher elevations of western part, a less common summer resident, sporadic in eastern (Boxford, Framingham, Wakefield); rare winter resident near coast. April 15-ХГау 26; summer; Sept. 1-Xov. 2t; winter. Eggs, June.

R. I.-Common migrant and rare winter resident. April 2SMay 10; Oct. 1-Nor. 1; winter.

Coxs.-Common migrant and uncommon winter resident; may breed at higher elevations (Litchfield, June 26, 1906). April 15-May 20 (June); Sept. 2.2-- Yor.; winter.

297. Spizella monticola (Gmelin) Baird.

\section{Tree sparrow; Winter chip-bird; Winter chippy.}

Chapman, Handb. birds east. No. Amer., 1595, p. 301; Birdlife, 1S9S, pl. 49 . 
PASSERES.

OSCINES.

FRINGILLIDAE.

Fringilla monticola Gmel., Sỵst. nat., 17ss, vol. 1, pt. 2, p. 912. "IN ANIERICA SEPTENTRIONALI."

Arctic thickets in summer; ficlds and marshes in winter migration; nests on the ground.

ME.- Common migrant and in southern counties a winter resident. (Early Aug., Fort Kent; Sept.) Oct. S-April $2 S$ (May 16).

N. H.-Common migrant, and from White Mts. south, uncommon winter resident. Oct. S-April 24.

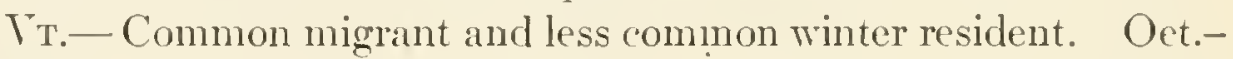
April.

MAss. - Common migrant and less common winter resident. (Sept. 21) Oct. 16-April 2S (May 7, 1S93, Lexington).

R. I.-Common winter resident. Nor. 7 -April 15.

Conx.-Common migrant and less common winter resident. Oct. 2S-April 12.

298. Spizella passerina (Bechstein) A. O. U. comm.

\section{Chipping sparrow; Chip-bird; Chippy; Hair-bird.}

Fringilla passerina Bechstein, in Latham, Allgem. nebers. vögel, 1798, vol. 3, pt. 2, p. 544, pl. 120, fig. 1. "CANad.."

Spizella socialis (Wils.). Chapman, Handb. birds east. No. Amer., 1895, p. 302. Eggg, Capen, 18\$6, pl. 10, figs. 1, 2.

Fringilla sociatis Wils. Audubon, Ornith. biogr., 1839, vol. 5, p. 517, pl. 104 .

Orchards, fields, and ricinity of dwellings; nests in trees, hedges, or vines.

Me.- Common migrant and summer resident. (April 2) April 9-Oct. 23 (Nov. 7). Eggrs, May 23-June 2; early July (2d brood).

N. H.- Common migrant and summer resident at lower elevations. April 10-Oet. 31. Eggs, May 31-June 16; July 29 (2d brood).

VT.-Common migrant and summer resident. April 4-Oct. 
PASSERES.

FRINGILLIDAE.

Mass.-Common migrant and summer resident. (Mar. 13, 24) April 5-Nor. 25 (Dec. 31, 1869, Watertown). Eggs, May $12-$ June 26 (2d brood ?).

R. I.-Common migrant and summer resident. April 4-Oct. 17. Eggs, May 19-June 15.

Coxs.-Common migrant and summer resident; rare winter resident. April-Nor. (winter).

299. Si'IZella BREweri Cassin.

\section{Brewer's sparrow.}

Cass., Proe. acad. nat. sci. Phila., 15う6, vol. S, p. 40. "Westery North Auerica, Califorvia, New Mexico." Ridgway, Bull. 50, L. S. nat. mus., 1901, pt. 1, p. 326.

Emberiza pallida Swains. Audubon, Birds of Amer., 1841, rol. 3, pl. 161 (fide Ridgway).

Prairies; nests on the ground.

Mass.-Accidental visitor: Watertown, Dec. 15, 1873.

300. Spizella pusilla (Tilson) Bonaparte.

\section{Field sparrow; Ground sparrow; Huckleberry-bird.}

Ridgway, Bull. 50, U. S. nat. mus., 1901, pt. 1, p. 31S. Egg, Capen, 1S56, pl. 10, figs. 3, 4.

Fringilla pusilla Wils., Amer. omith., 1S10, vol. 2, p. 121, pl. 16, fig. 2. "Pextspluania.... North and South Carolina... Georgia." Audubon, Ornith. biogr., 1S34, vol. 2, pl. 139.

Bushy fields; nests on the ground or in low bushes.

ME.- Uncommon summer resident in southern counties. April 7-Oct. 1.

N. H.- Locally common summer resident north to White Mit. valleys April 3-Oct. Egggs, June 12.

YT.-Locally common summer resident. April 4-fall (Dec. 25, 1908, Clarendon). Eggs, July 10. 
PASSERES.

OSCINES.

FRINGILLIDAE.

Mass.- Common summer resident and near the coast a rare minter resident. (Mar. 29) April 1-Nor. 13; (winter). Eggs, May S-June 12.

R. I.-Common summer and rare winter resident. April-Oct. 23; (winter). Eggs, May 19-June 11.

Cons.-Common summer and rare winter resident. (Late Mar.) April 6-Dec. 2 (winter, Hartford, New Haven). Eggs, Jume 22.

301. Junco Hremalis (Limné) Sclater.

Slate-colored Junco; Black snowbird; Blue snowbird; Junco; Snowbird; White-bill.

Chapman, Handb. birds east. No. Amer., 1895, p. 304; Birdlife, 1S9S, pl. 4S. Egg, Capen, 1SS6, pl.9, figs. 14, 15.

Fringilla hyemalis Linné, Syst. nat., ed. 10, 175S, vol. 1, p. 183. "ANERICA."

Thiekets, weedy and brushgrown fields; nests on the ground, rarely in bushes.

Me., N. H.- Common migrant and mainly in northern part, a common summer resident; rare winter resident in southeru part Egrgs, May 7-June 29; July 19 (2d brood).

TT.-Common migrant, less common summer and winter resident.

Mass. - Common migrant, less common winter resident; uncommon summer resident in westeru highlands (Mts. Holyoke, Nonotuck, Tom, Wachusett, Berkshires), sporadic in east (? Boxford, Medford, Wellfleet). Mar. S-May 21; summer; Sept. 3Nor. 30; winter. Eggs, -(Aug. 6).

R. I.-Common migrant and winter resident. Oct. 2-April 4 (May 19).

Coxs.-Common migrant and winter resident; rare loeal summer resident (Bear Mt., Salisbury, 2354 ft.). Oct. S-May 3 (summer). 
PASSERES.

OSCINES.

FRINGILITDAE.

302. Junco hyemalis moxtanus (Ridgway) A. O. U. comm.

Montana Junco.

Juneo montanus Ridgw., Auk, 1898, vol. 15, p. 321. "Columbia Falus, Montana." Bull. 50, U. S. nat. mus., 1901, pt. 1, p. 289.

Edges of thickets; nests on the ground.

Mass.-Aecidental visitor: Watertown, Mar. 25, 1874 (this specimen has been recorded as $J . h$. shufeldti and $J$. h. oregonus).

303. Melospiza ielodia (Wilson) Baird.

\section{Song sparrow; Bush sparrow; Ground-bird; Ground sparrow.}

Fringilla melodia Wils., Amer. ornith., 1810, vol. 2, p. 125, pl. 16, fig. 4. "Lxited States." Audubon, Birds of Amer., 1S 41 , vol. 3 , pl. 189.

IClospiza cinerea melodia (Wils.). Ridgway, Bull. 50, U. S. nat. mus., 1901, pt. 1, p. 35t. Egg, Capen, 1886, pl. 9, fig. 10-13.

Swamps, marshes, weedy fields; nests on the ground, in bushes, or even hollow trees.

XE.-Common migrant and summer resident; ratre winter resident (Calias, Cape Elizabeth, Gardiner). Mar. 7-Nor. (winter). Eggs, (April) Mar 20-June 7 ; July (2d brood).

․ H.- Common migrant and summer resident at lower elcvations; rare winter resident near coast. Mar. 9-Dec. (winter). Eggs, May 24-June 22; July 27 (2d brood).

$V_{\mathrm{T}}$ - C Common migrant and summer resident; rare winter resident in southern part (Bethel, Brattleboro, Brownsville, Taftsville). Mar. 7 -fall (winter).

MAss.-Common migrant and summer resident; less common winter resident at lower elevations. Mar. 10-Nor. 15; winter. Egrgs, April 30-July 29 (2d brood).

R. I.-Common migrant and summer resident; less common winter resident. Mar. 1-Nor. 14; winter. Egrgs, May 7-10.

Coxs.-Common migrant and summer resident; less common winter resident. Eggs, May 2t-(Aug. 20). 
PASSERES.

OSCINES.

FRINGILLIDAE.

304. Melospiza lincolnil (Audubon) Baird.

\section{Lincoln's sparrow; Lincoln's finch.}

Fringilla lincolnii Aud., Ornith. biogr., 1834, vol. 2, p. 535, pl. 193. "LABRADOR."

Pencaea lincolnii Aud., Birds of Amer., 1841, rol. 3, p. 116, pl. 177.

Edges of dense thickets and tangled growth; nests on the ground.

NE.-- Rare spring and uncommon fall migrant; rare summer resident in extreme north (Ft. Kent; $\sigma^{7}$ said to have been taken at Boothbay, July 14, and four birds at Bobson's Id., Aug. 9). May 12-.June 1; (summer); Sept. S-Oct. 13.

N. H.- Rare spring and uncommon fall migrant (once reported in summer, Nubanusit Lake, July 28, 1900, Thayer). May 1326; (? summer); Sept. 7-Oct. 4.

IT.-Rare migrant.

NAss. - Rarc spring and uncommon fall migrant. May $7-$ June 1; Sept. 12-Oct. 14 (Nor. 1).

R. I.- Rare migrant, mainly in fall. (Spring); Sept. 27.

Coxx-Rare spring and uncommon fall migrant. May 23; Sept. 21-Oct. 3.

305. Melospiza georgiana (Latham) Ridgray.

\section{Swamp sparrow.}

Ridgwar, Bull. 50, U. S. nat. mus., 1901, pt. 1, p. 382.

Fringilla georgiana Lath., Index ornith., 1790, vol. 1, p. 460. "Cuni praecedente," = "IN Georglae anericanae interioriBUS."

Fringilla palustris Wils. Audubon, Ornith. biogr., 1539, vol. 5, pl. 6t. Egg, Capen, 1886, pl. 9, fig. $7-9$.

Swamps, marshes, and old fields; nests on the ground.

ME.- Lncommon migrant and local summer resident. (Mar. 20, Bangor) April 6-Nov. 15. Eggs, May 31-June 3. 
PASSERES.

OSCINES.

FRINGILLIDAE.

N. H., VT.-Common migrant and less common local summer resident at lower elevations. April-Oct. 25.

MAss.-Common migrant and local summer resident; rare winter resident near the coast (to Beverly, Fresh Pond, Cambridge). Mir. 27-Nov. 30; winter. Egggs, May 13-July 14.

R. I.-Common migrant and less common local summer resident; may winter locally. April 4-Nor.14 (Dec. 25). Eggs, May 24-31.

Coxs.-Common migrant and less common local summer resident; rare winter resident. April 5-Nov. 20 (winter).

306. Passerella Iliaca (Merrem) Swainson.

\section{Fox sparrow; Fox-colored sparrow.}

Fringilla iliaca Merr., Beitr. z. besond. gesch. d. vögel, 17\$6-87, vol. 2, p. 40-41, pl. 10. "Nord-Amerika." Audubon, Ornith. biogr., 1\$35, vol. 2, p. 58, pl. 10 S.

Thickets and edges of woods; nests on the ground or in low trees.

Me.-Common migrant; accidental in winter. Mar. 15-April 17 (May 15); Oct. S-Nor. $2 S$ (Dec. 2, 9, 17, Seguin Id.; Jan. 24, 1909, Cape Elizabeth; Feb.).

N. H.-Common migrant. Mar 16-April 23; Oet. 5-Yor. 13. VT.-Common migrant. Mar. 15-April 5; fall.

MAss.-Common migrant and rare winter resident near the coast. (Feb. 26, 1906, Springfield; Mar. 3) Mar. 9-April 25 (May 2); (Sept. 17, Oct. 2) Oct. 15-Nov. 29 (Dec. 6 and winter).

R. I.-Common migrant and oceasional winter resident. Mar. 19-April 20 (May 21); Oct. 13-Nov. 27 (winter).

Conv.- Common migrant, and rare winter resident. Mar. 6April 19; Oet. 10-Nor. $2 S$ (winter).

307. Pipllo ervthrophthalmes (Limné) Vieillot.

\section{Towhee; Chewink; Ground robin; Marsh robin; Pewink.}

Chapman. Handb. birds east. No. Amer., 1995, p. 310; Birdlife, 1898, pl. 55. Eggg, Capen, 18\$6, pl. 10, fig. 13-15. 
PASSERES.

OSCINES.

FRINGILLID IE.

Fringilla erythrophthalma Linné, Syst. nat., ed. 10, 1755, vol. 1, p. 180. "Avierica."

Thickets and sprout land; nests on the ground, rarely in bushes.

Ne.- Lncommon summer resident in southwestern counties. May 6-Oct. 1s (Nov, 21).

N. H.-Common summer resident in southern part, becoming uncommon north to White Mt. valleys. May 1-Oet. 15.

T.-- Uncommon summer resident. May-Sept.

MAss.- Common summer and very rare winter resident. (April 2) April 1S-Oct. 22 (Nov. 30, Dec. 4); (winter). Eggs, May 17June 12 .

R. I.-Common summer and very rare winter resident. April 19-Oct. 13 (Jan. 14, 1896, Newport). Eggs, May 21-June 3.

Cons.-Common summer and very rare winter resident. April 25-Nor. 25 (Dec. 28, 1908, New Haven; Jan. 22, 1876, Portland). Eggs, Nay 21-June 6.

30S. Catidinalis cardinalis (Linné) Lichtenstein.

\section{Cardinal; Cardinal grosbeak; Red-bird; Virginia nightingale.}

Loxia cardinalis Linné, Sysst. nat., ed. 10, 1758, rol. 1, p. 172. "IN Alierica septentrionali."

Pitylus cardinalis Linné. Audubon, Birds of Amer., 1S41, rol. 3, p. 19S, pl. 203. Egg, Capen, 1S56, pl. 10, figs. 11, 12.

Bushy fields and thickets; nests in bushes.

ME.-Several recorded; probably some are escaped cage birds (f from flock of three, Gardiner, Dec. 19, 1895).

Vт.- Rare visitor or escape: Brattleboro.

MAss.-Occasional visitor at all seasons and escape; doubtfully stated to have bred at Cambridge (a wild bird and a freed cage bird nested in Brookline, eggs, June 6, 1S9S).

Cons.- Occasional risitor or escape. 
PASSERES.

OSCINES.

FRINGILLIDAE.

309. 7amelodia lutoviciasa (Linné) Coues.

\section{Rose-breasted grosbeak; Throat-cut.}

Loxia ludoviciana Linné, Syst. nat., ed. 12, 1766, rol. 1, p. 306. "In Lunovicia."

Habia ludoviciana (Linné). Chapman, Handb. birds east. No. Amer., 1895, p. 312; Bird-life, 189S, pl. 54; Stone, Auk, 1899, vol. 16, pl. 4. Egg, Capen, 1886, pl. 10, figs. 9, 10.

Orehard and shade trees and borders of woods near water; nests in bushes and trees.

Me.-Common summer resident except in eastern part. (April 26) May 4-Aug. 27. Eggrs, June 10-30.

N. H.- Common summer resident at lower elevations. May 12-Sept. 18.

VT.-Common summer resident. May-Sept. 14. Eggs, June $2-23$.

Mass.- Common summer resident. (April 26) May 2-Oct. 3. Eggs, May 25-June 15.

R. I.-Common summer resident, mainly in northern part. May 4-Oct. 2. Eggs, May 23-June 4.

Cons.-Common summer resident; accidental in winter. May 1-Sept. (Nor. 25; Jan. 1S, 1893, Stonington). Eggs, May $2 t$.

310. Guiraca Caerulea (Iinné) Swainson.

\section{Blue grosbeak.}

Chapman, Handb. birds east. No. Amer., 1895, p. 313. MeAtee, U. S. dept. agrie., biol. surv., bull. 32, pl. 4.

Loxia caerulea Linné, Syst. nat., ed. 10, 1758, rol. 1, p. 175. "America."

Brusb-grown fiekds and thickets; nests in bushes or weedy growths.

Me.-- Recorded from Calais, but in error for Grand Menan, N. B. 
PASSERES.

OSCINES

FRINGILLIDAE.

N. H.- Doubtfully recorded from East Derry, May 26, 1894.

VT.-Doubtfully recorded.

Mass-Accidental risitor: Brookline, May 29, 1880.

R. I.-Accidental visitor: Drownville, Oct. 12, 1899.

311. Passerina cranea (Linné) Vieillot.

\section{Indigo bunting; Indigo-bird.}

Tanagra cyanea Linné, Syst. nat., ed. 12, 1766, vol. 1, p. 315. "in Carolina."

Spiza cyanea Wils. Audubon, Birds of Amer., 1841, vol. 3, p. 96, pl. 170. Egg, Capen, 18s6, pl. 10, fig. 8 .

Brush-grown fields, thickets, and weedy tangles; nests among low bushes, brambles, or tall weeds.

Me.-Common summer resident in southern, and rare in northern counties (Caribou). May 11-Oet. 2. Eggs, Jume-July 15.

N. II.-Common migrant and summer resident at lower elevations. May 9-Oct. 1.

VT.-Common migrant and summer resident. May 18-fall. Eggrs, May 30.

Mass.-Common migrant and summer resident. May SOct. 29 (Dec. 5, 1871, Watertown). Eggs, June 3-22.

R. I.-Common summer resident, mainly in northern part. May 10-Oct. Eggs, Jume 1-11.

Coss.-Common migrant and summer resident. May 9-Oct. Eggs, June 16.

312. Spiza anericana (Gmelin) Ridgway.

Dickcissel; Black-throated bunting; Judas-bird.

Emberiza americana Gmel., Syst. nat., 17SS, vol. 1, pt. 2, p. $\$ 72$. "in Noveboraco." Audubon, Birds of Amer., 1841, vol. 3, p. 5S, pl. 156. Eggr, Capen, 1856, pl. 10, fig. 7 .

Grassy fields; nests on the ground or in low bushes. 
Me.-Aceidental risitor: Job's Id., Penobseot Bay, Sept. 29, 18St; St. George; Westbrook, Oct. 10, $18 S S$.

MAss. - Formerly an uncommon summer resident along coast and in western ralleys; now unknown. May 15-Oct. 3. Eggrs, June 9, 1877 (Medford)-Aug. 1, 1878 (2d brood, Hyde Park).

R. I.- Formerly a rale migrant: Newport, Sept. 25, 1 SSS.

Cons.- Formerly a summer resident, now unknown.

313. Calanospiza Melanocorys Stejneger.

\section{Lark bunting.}

Stejn., Auk, 1SS5, vol. 2, p. 49. Based on Townsend: "Plains of the Platte river."

Corydalina bicolor Towns. Audubon, Birds of Amer., 1St1, vol. 3 , p. 195 , pl. 202.

Prairies; nests on the ground.

Mass.-Accidental visitor: Lynn, Dec. 5, 1877.

\section{TANGARIDAE.}

314. Piranga ludovicuna (Wilson) Richardson.

\section{Louisiana tanager; Western tanager.}

Tanagra ludoviciana Wils., Amer. ornith., 1\$11, rol. 3, p. 27, pl. 20, fig. 1. "Lousiana."

Pyranga ludoriciana (Wils.). Audubon, Birds of Amer., 1841, rol. 3, p. 231, pl. 210.

Mountain forests; nests in trees.

ME.-Accidental visitor: near Bangor, about Oct. 1, 1889.

Mass.-Accidental visitor: Salem, Jan. 20, 1S7S.

Coxx-Accidental visitor: New Haven, Dec. 15, 1892.

315. Piranga erythromelas Vieillot.

Scarlet tanager; English robin (Berkshires); Fire-bird; Pocket-bird; War-bird.

Pyranga erythromelas Vieill., Nour. dict. d'hist. nat., 1S19, vol. 2S, p. 293. "AMí́rique." 
PASSERES.

OSCINES.

TANGARIDAE.

Pyranga rubra (Iinné). Audubon, Birds of Amer., 1St1, vol. 3, p. 226, pl. 209. Egg, Capen, 1S86, pl. 6, figs. 6, 7.

Deciduous woods, especially oaks and chestnuts; nests in trees.

Me.- Rare summer resident. May 12-Oct. 5.

N. H.-- Uncommon summer resident, mainly below $2000 \mathrm{ft}$. south of White Mts. May 13-Sept. 25.

VT.--Uncommon summer resident. May-fall.

MAss.-Common summer resident. (April 30, May 4) May SOct. 16. Eggs, June 2-17.

R. I.- Common summer resident, mainly of northern part. (April 4, 1891, Apponaug) May 4-Oct. 9. Eggrs, May 22-June 5.

Cosx.-Common summer resident. May 10-Sept. 11. Eggos, May 31-June 17.

316. Piranga rubra (Limné) Vieillot.

\section{Summer tanager; Summer redbird.}

Fringilla rubra Linné, Syst. nat., ed. 10, 1758, vol. 1, p. 181. "Anerica."

Pyranga aestiva Gmel. Audubon, Birds of Amer., 1S41, vol. 3, p. 222, pl. 208.

Open deciduous and mixed woods; nests in trees.

Me.-Accidental risitor: Portland, May 18, 1906; Wiscasset.

N. H., VT.-Doubtfully recorded.

MAss.-Accidental summer visitor. April 21, May, June, July, Aug.

R. I.-Accidental summer visitor: Providence (two seen); Seaconnet, April 27, 1901; Ten Mile River.

Cons.-Rare visitor. April, July.

\section{HIRUNDINIDAE.}

317. Progne stizis (Linné) Baird.

Purple martin; Black martin; Cape Cod swallow; House martin; Martin. 
Hirundo subis Linné, Sịst. nat., ed. 10, 1758, vol. 1, p. 192. “AD sinum Hunsonis."

Hirmulo purpurea Iinné. Audubon, Birds of Amer., 1S40, vol. 1, p. 170, pl. 45. Egg, Capen, 1S86, pl. 7, fig. 1.

Open country and ricinity of dwellings; nests in carities or bird houses.

ME.- 'Till recently a common local summer resident. April 22Sept. 4 (Sept 30). Eggs, June 5-July 4.

N. H.- Local summer resident north to White Mt. valleys; now uncommon. April 19-Sept.

VT. - Till recently a common local summer resident. April 25Sept. 20. Eggs, -June 23.

Mass. - Till recently a common local summer resident. April 14-Sept. 30. Eggs, Mily 30-June 21.

R. I.- Uncommon migrant and summer resident; formerly more common. April 25-Oct. S. Eggs, May 25-June 4.

Coxs.- Uncommon migrant and local summer resident; formerly more common. April 17-fall. Eggs, May 21.

31S. Petrochelidon lunifrons (Say) Cassin.

\section{Cliff swallow; Eave swallow; Mud swallow; Republican.}

II irundo lunifrons Say, Long's Exped. to Rocky Mts., 1823, vol. 2, p. 47. "Rocky Mountates, about the sources of the Arkansa and Platte."

Hirundo fulva Vieill. Audubon, Birds of Amer., 1S40, vol. 1, p. 177, pl. 47. Egg, Capen, 1S\$6, pl. 6, fig. 11-13.

Open country; nests in colonies under clifts or eaves of outbuildings.

Me.- Common migrant and summer resident. (April 11, Bangor) April 21-Sept. 6 (Sept. 19). Eggs, June 1-July 2.

N. H.- Common migrant and local summer resident. April 2SSept. 1. 
PASSERES.

OSCINES.

HIRUNDINIDAE.

YT.-Common migrant and summer resident. Late Aprilsummer.

MAss.- Common migrant and less common loeal summer resident. April 23-Sept. 4 (Sept. 30). ' Eggs, May 25-July 20.

R. I.- Uncommon migrant and summer resident. April 2SSept. 15. Eggs, Nay 31-July 16.

Cons.-Common migrant and less common local summer resident. (April 15) April 20-Sept. Eggrs, May 26- ? (2 broods).

319. Hirundo Erithrogaster Boddacrt.

\section{Barn swallow; Fork-tailed swallow.}

Bodd., 'Tabl. d. planeh. enlum. d'hist. nat., 1783, p. 45. Description in Buffon: "CAYENNE."

Hirundo rustica Linné. Audubon, Birds of Amer., 1S40, vol. 1, p. 1S1, pl. 4S. Egg, Capen, 1S\$6, pl. 6, figs. 8, 9.

Open country; nests in crevices of eliffs, but now generally on rafters of old barns.

Me.-Common migrant and summer resident. April 19-Sept. 19 (Oct. 29, Seguin Id.; Oet. 31, 1907, Portland). Eggs, May 2SJune 2 t.

N. H.- Common migrant and summer resident at lower elevations. April 20-Sept. 21.

VT.-Common migrant and summer resident. April 25-fall.

Mass.-Common migrant, less common summer resident. (April 5, 1S93, Waltham) April 17-Sept. 30 (Oct. 7). Eggs, May 1S-July 12.

R. I.-Common migrant and summer resident; still breeds in cliffs at Middletown. (April 10)-Sept. 23. Eggs, May 1S-30.

Cons.-Common migrant and summer resident. April 21Sept. Eggs, (! Sept. 3).

320. IrIDOPROCNE BICOLOR (Vieillot) Cones.

Tree swallow; Little martin; White-bellied swallow.

Hirundo bicolor Vieill., Hist. nat. ois. Amér. Sept., 1S07, rol. 1, 
p. 61, pl. 31. "États-Unis." Audubon, Birds of Amer., 1840, rol. 1, p. 175, pl. 46 . Egg, Capen, 1886, pl. 6, fig. 10.

Open country, especially near water; nests in cavities of dead trees or in birl boxes.

Me.-Common migrant and summer resident. (Mar. 19) April 4-Sept. 21. Egggs, May 30-June 11.

N. H.- Common migrant and less common summer resident at lower clevations. Mar. 23-Sept.

$V_{T}$.- Common migrant and less common summer resident. Eggs, June 1.

Mass.-Common migrant and less common summer resident. (Mar. 15) Mar. 22-Oet. 17 (Nov. 2). Eggs, May 14-June 15.

R. I.-Common migrant and less common summer resident; casual in winter. (Mar. 12) Mar. 28-Oct. 18. Eggs, May 2430.

Cosx.-Common migrant and less common summer resident. Mar. 25-Oct. 17 (Oct. 22). Eggrs, June.

321. Riparia Riparia (Linné) Sharpe and Wyatt.

\section{Bank swallow; Sand martin or swallow.}

IIirundo riparia Linné, Syst. nat., ed. 10, 1758, vol. 1, p. 192. "in Europae coldibus arenosis abruptis." Audubon, Birds of Amer., 1840, vol. 1, p. 187, pl. 50. Egg, Capen, 1886, pl. 6, fig. 14.

Open comntry near rivers and lakes; nests in colonies in burrows dug in sandy bluffs.

Me.-Common migrant and summer resident. (April 19) April 25-Sept. 1 (Sept. 19). Eggrs, May 29-June 24.

N. H.-Common migrant and local summer resident. MayAug. 24. Eggs, May 26.

T. - Common migrant and local summer resident. April 28summer. 
PASSERES.

OSCINES.

HIRUNDINIDAE.

MAss. - Common migrant and local summer resident. April 24Sept. 2 (Nov. 2). Eggs, May 2S-June 17.

R. I.-Common migrant and local summer resident. April 25-Aug. 29. Eggs, May 26-June 6.

Coxs.- Common migrant and local summer resident. April 21-summer.

322. Stelgidopterix serripennis (Audubon) Baird.

\section{Rough-winged swallow.}

Chapman, Handb. birds east. No. Amer., 1595, p. 322, fig. 90. Egg, Capen, 18s6, pl. 6, fig. 15.

Hirundo serripennis Aud., Ornith. biogr", 1S3S, vol. 4, p. 593. "A few mles from Bayou Sara," La. Aud., Birds of Amer., 1840 , vol. 1 , pl. 51 .

Open country near water; nests in holes in banks, or in cavities of eliffs and walls.

N. H.- Rare summer resident: near Hanover, 1905, 1906.

VT.-Rime summer resident (noted at Bennington; Lunenburg; Normich, May 6, 1905, April 29, 1906; West Danville, June 11, 1901; White River Junction). April 29-summer. Eggs, May 16.

MAss.- Rare local summer resident in western part (Glendale, young, 1906; Longmeadow, nesting, May, 1906; North Adams, two nesting pairs, June, 1895). Nay-summer.

Conv.- Rare local summer resident. (April 12) May 9-summer. Egrgs, May 26-June 9.

\section{BOIIB YCILLIDAE.}

323. Bombrcilla garrula (Linné) Vieillot.

\section{Bohemian waxwing.}

Bonaparte, Amer. ormith., 1S2S, vol. 3, p. 14, pl. 16, fig. 2. Egg, Ibis, 1861, ser. 1, vol. 3, pl. 4, fig. 1-6. 
PASSERES.

OSCINES.

BOMBICILLIDAE.

Lanius garrulus Linné, Syst. nat., ed. 10, 175S, vol. 1, p. 95. "In Europa \& America boreali ; astivat supra Srecian." Evergreen forests; nests in trees.

Me.- Rare and irregular winter visitor. Dec. 6-Feb. 6.

N. H.- Rare winter visitor: Hampton Falls, Feb. 17 and 21, 1S97; Nashua, 1 SSO.

VT.-Rare winter visitor: Brattleboro, April, 1901; Burlington (bis); St. Albans (bis). Nor. 25-April.

Mass.- Rare winter visitor: about six authentic records. Dee. 26-Feb. 18.

Coxs.-Accidental winter visitor: East Windsor Hill, near Hartford; ? New Haven, Feb. 11, 1875.

324. BOMBYCILla CEDRoRum Vieillot.

\section{Cedar waxwing; Apple-bird; Canada robin; Cedar-bird; Cherry-bird.}

Vieill., Hist. nat. ois. Amér. Sept., 1S07, rol. 1, p. 3S, pl. 57. "EN Auérique deputs le CaNada Jusqu'a Mexique."

Ampelis cedrorum (Vieill.). Chapman, Handb. birds east. No. Amer., 1895, p. 323; Bird-life, 1898, pl. 57. Egg, Capen, 1856, pl. 7 , figs. 2, 3 .

Orchards, shade trees, and woods; nests in trees.

ME.-Common migrant and summer resident; rare in winter. Eggs, June 29.

N. H.- Common migrant, and at lower clerations a common summer resident; rare in winter in southern part. Egrg, July 29.

VT.-Common migrant and summer resident; uneommon in winter in southern part.

Mass.- Common migrant and less common summer resident; irregularly common in winter but generally absent in Nov. and Dec. Eggs, May 30-July.

R. I.-Common migrant and summer resident; irregular in winter. Eggs, June 7-20. 
PASSERES.

OSCINES.

$$
\text { BOMBICILLIDAE. }
$$

Cons.-Common migrant and summer resident; irregular in winter. Eggs, June 20-Aug. 1 (Aug. 27).

\section{LANIIDAE.}

325. Lanius borealis Vieillot.

\section{Northern shrike; Butcher-bird.}

Vieill., Hist. nat. ois. Amér. Sept., 1S07, vol. 1, p. S0, pl. 50. "Canada et sur-tout la Baif n'Hudson." Audubon, Birds of Amer., 1842, vol. 4, p. 130, pl. 236.

Open country and edges of woods; nests in trees.

ME.-Uncommon winter resident; supposed breeding records refer to L.l.migrans. Oct. 15-April 17.

N. H.- Uncommon winter resident at lower elevations. Nov. 12-April.

VT.- Uncommon winter resident. Fall-April 13.

MASs.-Uneommon winter resident. (Oct. 6) Oct. 12-April 16 (May).

R. I.- Uneommon, irregular winter resident. Nov. 2S-Mar. 23.

Cons.- Irregular winter resident, sometimes common. Oet. 29-Mar. 24 (April 11).

326. Lanius ludovicianus Linné.

\section{Loggerhead shrike.}

Linné, Syst. nat., ed. 12, 1766, vol. 1, p. 134. "IN Ludovicia." Ridgway, Bull. 50, U. S. nat. mus., 1904, pt. 3, p. 241. Audubon, Birds of Amer., 1S42, vol. 4, pl. 237.

Open country; nests in trees.

Cons.-Aceidental visitor: South Norwalk, Feb. 17, 1905 (J. A. Allen). 


\section{LANIIDAE.}

327. Lanies icdovichanes migrans IV. Palmer.

Migrant shrike; Butcher-bird; Cricket hawk; Grasshopper hawk; Joree; Mouse hawk.

IV. Palmer, Auk, 1598, vol. 15, p. 24S. "Kingston, Oxtario." Egg, Cilpen, 1SS6, pl. 7, figs. 11, 12.

Orchards and open tree growtli; nests in trees.

Me.- Uncommon loeal summer resident. (Mar. 1) Mar 24Sept. (Nov. 17). Eggs, May 5-25.

X. H.-Uneommon summer resident in valley country; aceidental in winter. Mar. 20-Sept. (Jan. 20, Concord). Eggs, April 26-May 5.

VT.- Uncommon local summer resident. Mar. 3-fall. Eggs, April 2S-May 23.

MAss. - Rare migrant and in westem part, rare summer resident; very rare in winter. Mar. 29-Nov. 27 (Dec. 19, Jan., Feb.). Eggs, May 10 (Williamstown).

R. I.- Rare migrant. (? Spring); Aug. 29-Sept. 2.

Coxn.- Rare migrant and winter resident (Portland, Nov. 1876; Saybrook, Jan., 1S79); possibly a rare summer resident (MIt. Carmel, May 24, 1873). Spring-(May 24); Aug. 29-winter.

\section{VIREONIDAE.}

32S. Vineosylia oldvacea (Linné) Bonaparte.

Red-eyed Vireo; Politician; Preacher; Red-eyed greenlet.

Muscicapa oliracea Limné, Syst. nat., ed. 12, 1766, vol. 1, p. 327. "IN America septentrionali."

Vireo olixaceus (Linné). De Kay, Zool. New York, 1St4, vol. 2, p. 124, pl. 36, fig. 79. Egg, Capen, 18s6, pl. 7, figs. 4, 5.

Orehards and deciduous shade and forest trees; nest pensile in trees or bushes. 
PASSERES.

OSCINES.

VIREONIDAE.

Me.-Common summer resident. (May 1) May 7-Oet. 2 (Oct. 11). Eggs, -June 29.

N. H.-Common summer resident below $3000 \mathrm{ft}$. May \&Sept. 30.

VT.-Common summer resident. May 9-fall.

MAss.-Common summer resident. May 4-Oct. 11 (Oct. 26; Nov. 2, 1870, Watertown). Eggs, May 25-July 20.

R. I.-Common summer resident. (April 29) May-Sept. 24 (Oct. 11). Eggs, May 2S-June 6.

Coxx.- Common summer resident. May 6-Oet. 2. Eggs, June 1-12.

329. Vireosylya philadelphica Cassin.

\section{Philadelphia Vireo; Brotherly-love Vireo.}

Cass., Proc. acad. nat. sei. Phila., 1851, rol. 5, p. 153, pl. 10, fig. 2. "xear Philadelphia."

Yireo philadelphicus (Cass.). Dwight, Auk, 1897, vol. 14, p. 269, pl. 2. Egg, Brewster, Auk, 1905, vol. 22, pl. 11.

Open deciduous growth, especially of birch and poplar; nest pensile in trees or bushes.

NE.- Uncommon summer resident in northern part (Bucksport, Lake Umbagog). Mary 21-Oet. 2. Eggs, June 15.

N. H.- Uncommon summer resident north of White MIts., elsewhere rare migrant. May 26-Sept. 29.

VT.-Rare migrant: Breadloaf, Sept. 19, 1900; Morrisville; Pittsford, Sept. 11, 18s9. (Reported nesting at Brattleboro, June, 1901.) May; Sept. 11-19.

Mass.- Rare migrant. May (? 10)-17; Sept. 7-27.

R. I.- Rare migrant: Drownville, May 31, 1891; West Greenwich, Мay 21, 1902.

Coxx.- Rare migrant: New Haven, May 19, 18ss; Portland, Sept. 21, 1593, Sept. 17, 1894; (? Stratford, "Vireo gillus.... under parts.... mostly bright yellow"). 
PASSERES.

OSCINES.

VIREONIDAE.

330. Vireosylua ginta (Vieillot) Cassin.

\section{Warbling Vireo.}

IM uscicapa gilea Vieill., Hist nat. ois. Amér. Sept., 1S07, vol. 1, p. 65, pl. 3.. "L'ETAT DE New-YorCk."

rireo gileus (Vicill.). Chapman, Handb. birds "east. No. Amer., 1595, p. 329. Dwight, Auk, 1897, vol. 14, pl. 2. Egg, Capen, 18s6, pl. 7 , fig. 6 .

Orehard and shade trees, especially elms; nest pensile in trees.

Me.- Uneommon local summer resident. May 5-Sept. 15 (Oet. 7).

N. H.- Uncommon local summer resident north to White Mt. valleys. May $1-$ Sept. 17.

VT.-Uncommon local summer resident. May 3-

Mass.- Common summer resident. (April 24, 190S, Auburndale) April 29-Sept. 26. Eggs, May 30-June 11.

R. I.-Common summer resident, especially of village shade trees. May 5-Sept. 25. Eggs, May 2\&-30.

Coxx-Common summer resident. (April 20, 1S9S, Jewett City) April 29-Oet. 13 (Oct. 25, 1900, Hartford). Eggs, June $2-17$.

331. Lanivireo flavifroxs (Vieillot) Lawrence.

\section{Yellow-throated Vireo.}

T'ireo flarifrons Vieill., Hist. nat. ois. Amér. Sept., 1807, vol. 1, p. S5, pl. 54. "Ëtats-Unis." Audubon, Birds of Amer., 1St2, vol. 4, p. 141, pl. 238. Egg, Capen, 18S6, pl. 7, figs. 7, S.

Orehard and shade trees and edges of woods; nest pensile in trees.

Me.- Rare summer resident in southwestern counties. Mar. 12-Sept.

N. H.- Uncommon summer resident beeoming rare north to White MIt. valleys. May S-Sept. Eggs, June 14. 


\section{VIREONIDAE.}

VT. - Uncommon summer resident (East Bethel).

MAss.-Common summer resident becoming local in western part. May 1-Sept. 19. Eggs, May 24-June 17.

R. I.-Common summer resident. May 1-Sept. Eggs, May 24-June 2.

Coxx-Common migrant and summer resident. May 6Sept 17. Egegs, Maly 21-June 11.

\section{I ANIVIREO solitarius (Vilson) Allen.}

\section{Blue-headed Vireo; Solitary Vireo.}

Muscieapa solitaria Wils., Amer. ornith., 1S10, vol. 2, p. 143, pl. 17, fig. 6. "Near Philadelphia."

Virro solitarius Vieill. Audubon, Birds of Amer., 1S42, vol. 4, p. 144, pl. 239. Egg, Capen, 1S\$6, pl. 7, fig. 9.

Cool mixed woods; nest pensile in trees.

ME.- Uncommon summer resident mainly in northern part. (April 24) April 2S-Oet. 6 (Nor.).

N. H.- Uncommon summer resident up to $3000 \mathrm{ft}$. April 29Oct. 7. Eggs, May 29-late June.

Y'T.- Uneommon summer resident.

MAss.- Uncommon migrant and rare summer resident, becoming local in south and east. (April 11, 190S, Stoughton) April 14-Oct. 17. Eiggs, May 13- June 26.

R. I.- Uncommon migrant and rare loeal summer resident (Washington, West Greenwich). April 23-Oct. 22. Eggs, May 15June 15 .

Coxs.- Uncommon migrant and rare summer resident (Eastford, near New Haven, Norfolk, West Simsbury). Late April-Oet. 13. Eggs, May 27-June 23.

333. Tireo griseus (Boddacrt) A. O. U. comm.

\section{White-eyed Vireo; White-eye.}

Tanagra grisea Bodd., 'l'abl. d. planch. enlum. d’hist. nat., 17S3, p. 45. Based on Buffon. 
Vireo noveboracensis (Gmel.). DeKay, Zool. New York, 1St4, vol. 2, p. 122, pl. 36, fig. 78. Egge, Capen, 1886, pl. 7, fig. 10.

Bushy thickets; nest pensile in bushes.

ME.-Doubtfully recorded.

N. H.- Several insatisfactory records: Chesterfield, Manchester.

VT.-Accidental visitor: Brattleboro; ? St. Johnsbury, various records (Clark); Townshend, 1893.

MAss. - Rare local summer resident near coast and in southern valleys (Shefficld). May 2-Sept. 27 (Oct. 30). Eggos, May 22June 18 .

R. I.-Uncommon local summer resident. May 7-Sept. 18. Eggs, June 2, 3.

Cons.- Common summer resident in southern part and Connecticut valley. May 2-Sept. Eggs, June 9-12.

334. Vireo bellil Audubon

\section{Bell's Vireo.}

Aud., Birds of Amer., 1St4, vol. 7, p. 333, pl. 48.5. "Shores ANd pratries of the UpPer Missovri."

Bushy thickets; nest pensile in bushes or low trees.

N. H.-Aecidental visitor: Durham, Nor. 19, 1897.

\section{INIOTILTIDAE.}

335. Mniotilta varia (Linné) Vieillot.

Black and white warbler; Black and white creeper.

Cliapman, Warblers No. Amer., 1907, p. 39, pl. 2, figs. 4, 5. Eggg, Capen, 1S\$6, pl. 3, figs. 22, 3.

Motacilla varia Linné, Sỵst. nat., ed. 12, 1766, rol. 1, p. 333. "in Jamaica, Dominica."

Deciduous and mixed woods; nests on the ground. 
PASSERES.

OSCINES.

MNIOTILTIDAE.

ME.-Common migrant and local summer resident. (April 27) May 3-Oct. 5. Eggs, May 25-June 21.

N. H.-Common migrant and less common summer resident at lower altitudes. April 27-Sept. 29. Egges, June 24.

VT.-Common migrant and summer resident. May 5 -fall. Eggs, June 20.

MAss - Common migrant and less common summer resident. (April 1S) Apuil 2S-Oct. 29 (Dec. 5). Eggs, May 17-June 22.

R. I.- Common migrant and less common summer resident. (April 15) May 1-Oct. 7. Eggs, May 21, 23.

Cosv-Common migrant and less conmon summer resident. (April 20) April 25-Oct. 10. Eggs, May 20-Jume 4.

336. Protonotaria citrea (Boddaert) Baird.

\section{Prothonotary warbler; Golden swamp warbler.}

Chapman, Warblers No. Amer,, 1907, p. 54, pl. 3, fig. 3-4.

Iotacilla citrea Bodd., 'Tabl. d. planch. enlum. d'hist. nat., 1783, p. 44. Description in Bufion: "Loursiavie."

Bushy and tree-grown swamps; nests generally in cavities of stumps or dead trees.

Me.-Accidental risitor: Calais, Oct. 30, 1862.

VT.-Doubtfully recorded.

MAss.-Accidental visitor. May 1-Sept. 15.

R. I.-Accidental visitor: Lonsdale, April 29, 1592, April 19, 1S93; South Kingston, April 20 or 21, $18 S 4$.

337. Helintheros vermivorus (Gmelim) Bonaparte.

\section{Worm-eating warbler.}

Motacilla merimora [ = vermirora] Gmel., Syst. nat., 17SS, vol. 1, pt. 2, p. 951. "in Pexsilvania."

Helinaia vermivora Lath. Audubon, Birds of Amer., 1St1, vol. 2, p. 86, pl. 105 . Egg, Capen, 18s6, pl. 3, fig. 6. 
PASSERES.

OSCINES.

\section{MNIOTILTIDAE.}

Dry wooded slopes and ravines with undergrowth; nests on the gromind.

Me., N. H.- Unsatisfactorily recorded.

YT.-Accidental visitor: St. Albans, 1891.

MAss.-Accidental visitor: Cambridge, Sept. 19, 1SS1; Easthampton; Salem Willows, April 14-16, 1902; Taunton, May 9, 1890 .

Coxn.- Rare summer resideut. May 10-Aug. 22. Eggs, (New Haven, Saybrook) May 25-June 19.

335. Helmintmophila pinus (Linné) Ridgrway.

\section{Blue-winged warbler; Blue-winged yellow warbler.}

Chapman, Warblers No. Amer., 1907, p. 65, pl. 5, fig. 1-2. Egg, Capen, 1856, pl. 3, fig. 7 .

Certhia pinus Linné, Syrst. nat., ed. 12, 1766, vol. 1, p. 157. "in Alierica septentrionali."

Low woods and sprout land; nests on the ground.

VT.- Unsatisfactorily recorded.

MAss.- Very rare visitor. May 12-17 (? 29).

R. I.- Very rare summer resident: nest at Gloucester, May 30, 1890 .

Cons.- Locally common summer resident in southern part and Connecticut ralley. (May 2) May 9-late Aug. Eggs, May 20-June 20.

339. Hemanthophula chrisoptera (Linné) Ridgway.

\section{Golden-winged warbler.}

Chapman, Warblers No. Amer., 1907, p. 60, pl. 5, fig. 5-6. Egg, Capen, 1S\$6, pl. 3, fig. S.

Motacilla chrysoptera Linné, Syst. nat., ed. 12, 1766, rol. 1, p. 333. "in Pensyliania."

Low bushy growths and sprout lands; nests on the ground. 
PASSERES.

OSCINES.

\section{MNIOTILTIDAE.}

'N. H.- Rare in spring and early summer in southernmost part; may breed. May, June.

V'T.- Rare migrant; ?"rare summer resident" (Perkins). Spring; (Oet. 26, 1907, Woodstoek).

MAss.- Uncommon summer resident, mainly in eastern part. (May 4) May 7-Sept. 1 (Oct. S). Eggs, June 1-20.

R. I.- Rare summer resident.

Coxx.- Uneommon summer resident. (May 3) May 12Aug. 24. Eggs, May 29-June 4.

340. Helinthophila rubricapilla (Wilson) Faxon.

\section{Nashville warbler.}

Sylvia rubricapilla Wils., Amer. ornith., 1811, vol. 3, p. 120, pl. 27, fig. 3; vol. 6, p. xv. "lennesee... . Not far rrou NashvILLE."

Helinaia rubricapilla (Wils.). Audubon, Birds of Amer., 1841, vol. 2, p. 103, pl. 113. Egg, Capen, 1856, pl. 3, figs. 9, 10.

Open woodland and thickets bordering swamps; nests on the ground.

Me.-Common migrant and summer resident. (April 30) May 4-Sept. 27 (Oet. 4). Eggs, June 3-July 10.

N. H.- Common migrant and summer resident up to $4500 \mathrm{ft}$. (April 30) May 4-Sept. 13. Eggs, May 25-June S.

VT.-Common migrant and summer resident. May 3-fall.

MASS.-Common migrant and less common summer resident, accidental in winter. (April 20, 28) May 2-Sept. 25 (Oct. 14; Jan. 1890, Swampscott). Eggs, May 21-June 21.

R. I.-Common migrant and uncommon summer resident. May 4-Sept. 17. Eggs, May 22-June 1.

Coxs-Common migrant and uncommon summer resident. (April 22, 1889, Bridgeport) May 5-Sept. Eggs, May 30-June S. 
MNIOTILTIDAE.

341. Helminthophila celata (Say) Ridgway.

\section{Orange-crowned warbler.}

Chapman, Warblers No. Amer., 1907, p. 86, pl. 7, figs. 3, 4.

Sylvia celatus Say, Long's Exped. to Rocky Mits., 1S23, vol. 1, p. 169. "Engmeer Cantonment," Iowa.

Wooded and bushy country; nests on or near theground.

ME.- Unsatisfactorily accredited (Brunswick).

N. H.- Very rare migrant: Hollis, May 16, 1876 (nec Isles of Shoals).

MAss. - Very rare spring and late fall migrant; accidental in winter. May 15 (Springfield); Sept. 30-Nor. 29 (Jan. 1, 1875, Lymn).

R. I.- Very rare spring and late fall migrant: Cranston, Dec. 3, 1874; East Providence, May 9, 1891.

Conv.- Rare migrant. May S, $18 S S$ (East Hartford); Oct.Nov.

\section{Helminthophila peregrina (Wilson) Ridgway.}

\section{Tennessee warbler.}

Chapman, Warblers No. Amer., 1907, p. 83, pl. 7, figs. 1, 2.

Sylvia peregrina Wils., Amer. ornitl., 1811, vol. 3, p. \$3, pl. 25, fig. 2. "Banks of the Cumiserland river, ... Tennesee."

Dense thickets and edges of woods; nests near the ground.

Me.- Rare migrant and in northern part, a rare summer resident. Nay 15-26; summer. Eggs, June 4.

N. H.- Rare migrant and from White Mts. north, a rare local summer resident (Franconia, Lake Umbagog). May 20-Sept. 9.

VT.- Rare migrant and possible summer resident (Mt. Holly, two pairs, June, 1897 , above $2000 \mathrm{ft}$.).

MAss.- Rare migrant and possibly a very rare summer resident (Mt. Greylock, July 15 and 16, 1SSS; ? Springfield, very doubtful). May 10-30; (July 15, 16); Aug.-Sept. 10. 
PASSERES.

OSCINES.

MNIOTILTIDAE.

R. I.- Rare migrant. May 18; Sept. 18.

Coxn.- Rare migrant. May 19-27 (June S); Sept. 24(? Nov, Clark).

\section{Compsothlypis americana usneae Brewster.}

\section{Northern Parula warbler; Blue yellow-backed warbler; Usnea} warbler.

Brewst., Auk, 1896, vol. 13, p. 44. "Lake Unbagog, Manne." Chapman, Warblers No. Amer., 1907, p. 104, pl. S, fig. 1-3. Egg, Capen, 18s6, pl. 3, figs. 4, 5 .

Forests and open tree growth; nests in pendulous masses of Usnea lichen.

Me.- Common migrant and uncommon local summer resident. May 5-Sept. 30. Eggs, May 31-June 17.

N. H.-Common migrant and less common summer resident below $3000 \mathrm{ft}$. (April 30) May 3-Oct. 1. Eggs, May 31-June 12.

$V_{\mathrm{T}} \mathrm{r}$ - Common migrant and less common summer resident. May 2-fall.

MAss. - Common migrant and less common local summer resident. (April 25) May 1-Oct. 10 (Nov. 19, 1S81, Cambridge). Eggrs, May 30-July 5.

R. I.-Common migrant and uncommon local summer resident. April 30-Oct. 7. Eggs, June 2-5.

Cons.-Common migrant and uncommon local summer resident. May 2-Oct. 15 (Oct. 20). Eggs, (May 1S) May 29-June 22 (Aug.).

344. Dendroica tigrina (Gmelin) Baird.

\section{Cape May warbler.}

Chapman, Handb. birds east. No. Amer., 1595, p. 352. Egg, Capen, 1856 , pl. 4, fig. 15 .

Motacilla tigrina Gmel., Syst. wat., 17SS, vol. 1, pt. 2, p. 985. "Canada." 
PASSERES.

OSCINES.

\section{MNIOTILTIDAE.}

Sylricola maritima Wils. Audubon, Birds of Amer., 1S41, vol. 2, p. 4t, pl. Si.

Fortlern coniferous forests in summer, and open tree growtl in migration; nests in trees.

Me. - Rare migrant and in northern part a rare summer resident. May 6-2t; summer-Aug. 22.

N. H. - liare migrant and north of White Mts. a lare summer resident (Franconia, June 3). May 10-Sept.

VT.- Rare migrant and (?) summer resident (MIt. Killington, Ross).

MAss. - Rare migrant. (May 2) May 10-25; Aug. 1S-Sept. 15 (Oct. 9).

R. I.- Rare migrant. Nay 13-20; Sept.

Conx.- Rare migrant. May S-26; Ang. 2 S.

345. Dendroica aestria (Gmelin) Baird.

Yellow warbler; Cotton wren; Garden warbler; Golden warbler; Spy-about; Summer yellow-bird; Wild canary; Yellow-belly; Yellow-bird.

Motacilla aestiva Gmel., Syst. nat., 17SS, vol. 1, pt. 2, p. 996. "in Gujana, aestate in Canada."

Sylricola aestiva (Gmel.). Audubon, Birds of Amer., 1S 11 , rol. 2, p. 50, pl. SS. E.gg, Capen, 1S\$6, pl. 3, fig. 11-14.

Orchards, shade trees, and bushy swamps; nests in trees and bushes.

ME.-Common summer resident. (April 30) May 3-Sept. 10. Eggs, May 29-June 30 (July).

N. H. - Uncommon local smmmer resident of valleys and bottomlands; rare from White Mits. north. May +-Sept. 11. Eggs, June 7 .

YT. Common summer resident of valleys and bottomlands. May 4-Sept.

MAss.-Common migrant and summer resident. (April 23) 
PASSERES.

OSCINES.

MNIOTILTIDAE.

May 1-Sept. 30 (Nov. 28, 1891, Cambridge). Eggs, May 20June $1 \dot{4}$.

R. I.-Common summer resident. (April 17, 29) May 1-Sept. Eggs, May 25-June 4.

Coxv.- Common migrant and summer resident. (April 30) May 1-Sept. Eggs, May 20-.June 30.

346. Dendroica Caerulescens (Gmelin) Baird.

\section{Black-throated blue warbler.}

Chapman, Warblers No. Amer., 1907, p. 133, pl. 9, fig. 1-3. Egg, Capen, 1856, pl. 4, fig. 1.

Motacilla caerulescens Gmel., Syst. nat., 17Ss, vol. 1, pt. 2, p. 960. "IN INSULA S. Dominici."

Thickets and forest undergrowth; nests near the ground.

Ne.-Common migrant, and less common summer resident. May 4-late Oct. Eggs, June 10.

N. H.- Common migrant, and from White Mts. north a less common summer resident, becoming rare and local south. May 10Oct. 10. Eggs, June 19.

VT.-Uncommon summer resident in northern part. May 5fall.

Mass. - Common migrant and at higher elevations of western part (Granville, Russell, and north) a less common summer resident. May 5-June 3; summer; Aug. 26-Oct. 11 (Nor. 3, 19).

R. I.- Uncommon migrant. May 14-22; fall-Oet. 17.

Coxs.- Common migrant and rare summer resident (Eastford, Litchfield). (April 29) May 10-summer; Sept.-Oct. 19. Eggs, June $6-13$.

347. Dendroica coronata (Linné) Gray.

Myrtle warbler; Golden-crowned warbler; Willow warbler; Yellow-rumped warbler.

Chapman, Warblers No. Amer., 1907, p. 141, pl. 10, fig. 1-3. Egg, Capen, 1886, pl. 4, fig. 2-4. 
Motacilla coronata Limné, Syst. nat., ed. 12, 1766, rol. 1, p. 333. "PExsYliania."

Coniferous woods, and in migration, open tree growth; nests in trees.

Me.- Common migrant and sonth to Scarboro a less common summer resident; rare winter resident (Cape Elizabeth). April 15Oct. 30 (winter). Eggs, May 30-.June 11.

N. H.- Common migrant and summer resident, local south of White Mts. (Mar. 5, Mar. 20) April 23-May 2S; summer; Sept.-Oct. 23 (Nor.30). Egges, May 31-June 7.

IT.-Common migrant, and umeommon summer resident (south to Brattleboro). April 12-fall.

MAss.- Common migrant, and in higher parts of western counties an uncommon summer resident (to Mt. Wachusett); near the coast al regular local winter resident in small numbers. April 13May 30; summer; Aug. 21-Nov. 26; winter.

R. I.-Common nigrant and less common winter resident. Sept. T-May 10.

Coxs-Common migrant and uncommon winter resident. April S-May 20; Sept. 20-Nor. 10; winter.

348. Dexdroica acdeboni ('Tominsend) Baird.

\section{Audubon's warbler.}

Chapman, Warblers No. Amer., 1907, p. 147, pl. 10, fig. 4-6.

Sylvia auduboni Towns., Joum. acad. nat. sci. Phila., 1S37, vol. 7, p. 191. "Foriests of the COLumina river."

Coniferous woods; nests in trees.

Mass. - Accidental visitor: Cambridge, Nov. 15, 1876.

Coxx.- Doubtfully recorded: New IIaren, May 6 and $\&, 1893$ (A. H. Verrill).

349. Dexmroica magrolia (Wilson) A. O. U. comm.

Magnolia warbler; Black and yellow warbler; Spotted warbler. Sylvia magnolia Wilson, Amer. ornith., 1S11, vol. 3, p. 63, p]. 
PASSERES.

OSCINES.

MNIOTILTIDAE.

23, fig. 2. "Baxks of the Little Muan xear its Junction With the OHIO"; and "Not far fron Fort ADAMS ON THE Mississippl."

Dendroica maculosa (Gmel.). Chapman, Warblers No. Amer., 1907, p. 121, pl. 11, fig. 1-3. Eggg, Capen, 1886, pl. 4, figs. 12, 14.

Coniferous and mixed thickets near streams in summer, woodlands generally in migration; nests in low trees.

ME.-Common migrant and in northern part a less common summer resident. May 6-Sept. 26. Eggs, May 30-June 16.

X. H.-Common migrant; uncommon local summer resident south of White Mts., commoner north, to $3000 \mathrm{ft}$. May 9-Oct. 3. Eggs, May 24-June 20.

VT.-Common migrant, less common local summer resident. May 14-fall.

MAss.- Common migrant and in northwestern counties a less common local summer resident. (May 4) May 7-30; summer; Aug. 25-Oct. 9. Eggs, June 15.

R. I.-Common migrant. May 14-25; Sept.-Oct. S.

Coxx.-Common migrant. May 9-June 1; Sept. 15-Oct. 1.

350. Dendroica cerulea (Wilson) Baird.

\section{Cerulean warbler.}

Chapman, Warblers No. Amer., 1907, p. 170, pl. 9, fig. 4-6.

Sylvia caerulea Wils., Amer. ornith., 1810, vol. 2, p. 141, pl. 17, fig. 5. "Pennsylvania."

High tree tops; nests in trees.

Mass.-Accidental visitor: Cohasset, 187t.

R. I.-Accidental visitor: Lonsdale, May 14, 1893; Pawtucket, May 22, 1879; Providence, May 22, 1878.

Conv-Accidental visitor: Seymour, May 10, 18ss; Stratford, April, 1St1; Suffield, June 12, 1875. 
PASSERES.

OSCINES.

MNIOTILTIDAE.

351. Dendrolca pexsimanica (Limné) Baird.

\section{Chestnut-sided warbler.}

Chapman, Warblers No. Amer., 1907, p. 18т, pl. 12, fig. 4-6. Egg, Capen, 1886, pl. 4, figs. 11, 12.

Motacilla pensyleanica Linné, Sỵst. nat., ed. 12, 1766, rol. 1, p. 333. "in Pexsiluania."

Brushy thickets, clearings, and sproutlands; nests in low bushes.

ME.- Common migrant and summer resident. May 6-Sept. S. Eggs, June 4-July 4.

N. H.-Common migrant and summer resident, mainly at lower elevations. May 6-Sept. 25. Eggs, May 26-June 6.

VT.-Common migrant and summer resident. Eggs, June 6.

MAss.- Common migrant and summer resident. (April 2S, 1908, Stoughton) May 2-Sept. 20. Eggs, May 26--June 17.

R. I.- Common migrant and summer resident. May 1-Sept. 7. Eggs, May 22-June 4.

Cons.-Common migrant and summer resident. May t-Sept. Eggs, May 23-June 15.

352. Dendroica stritata (Forster) Baird.

\section{Black-poll warbler; Autumnal warbler.}

Chapman, Warblers No. Amer., 1907, p. 196, pl. 2, fig. 1-3. Egg, Capen, 1886, pl. 4, figs. 7, S.

II uscicapa striata Forst., Phil. trans. roy. soc. London, 1772, vol. 62, pp. 406, 428. "Severx River.... ad Sinum Hudsonis."

Spruce and fir forests in summer, and tree growth generally in migrations; nests in low trees.

Me.- Common migrant, and in northern part, a common summer resident. May 9-June 9; summer-Oct. 10. Eggs, June 15 -early July.

N. H.- Common migrant and from White MIts. north, a com- 
PASSERES.

OSCINES,

MNIOTILTIDAE.

mon summer resident mainly above 3000 ft. May S-Oct. 10. Eggs, June 28.

$V_{T}$.-Common migrant, and on higher mountains an uncommon summer resident (Killington Peak, Mt. Mansfield, Stratton Mt.).

MAss. - Common migrant; may breed rarely (young at North Adams, August). (April 15) May 8-June 14; (Aug. 17, 1886, Concord) Sept. 1-Nor. 7.

R. I.-Common migrant. May 10-31 (June 17); Sept. 3-Oct. 23.

Coxx.- Common migrant. May 11-June 5; Sept. 10-Oct. 26.

353. Dendroica blackburniae (Gmelin) Baird.

\section{Blackburnian warbler; Hemlock warbler.}

Chapman, Warblers No. Amer., 1907, p. 175, pl. 3, figs. 1, 2. Egg, Cilpen, 1886, pl. 4, figs. 5, 6.

Motacilla blachbumiae Gmel., Syst. nat., 1788, vol. 1, pt. 2, p. 977. "In Noveroraco."

High coniferous and mixed woods, especially hemlock and white pine; nests in tree tops.

Me.- Rare migrant in southern counties, elsewhere an uncommon local summer resident. (May 4) May 6-Sept. 17. Eggs, June 5-15.

N. H.- Uncommon local summer resident south of White Mts., thence commoner up to $3000 \mathrm{ft}$. (May 3) May S-Sept. 10. Eggs, June 4-15.

VT.-Uncommon local summer resident. (May 2) May isummer.

MAss.- Rare migrant and summer resident in eastern, commoner in northwestern part. (May 2) Mạ 6-30; summer; Sept. 7-23) (Oct. 10). Eggrs, June $\overline{5}-26$.

R. I.- Uncommon migrant. May 14; Sept.-Oct.

Coxx-Common migrant and rare summer resident. May $5-$ 27; summer; Sept. 15-Oct. 3 (Nor. S, 1907, New Haren). 
PASSERES.

OSCINES.

\section{MNIOTILTIDAE.}

354. Devdrolca Castanea (Wilson) Baird.

\section{Bay-breasted warbler.}

Chapman, Warblers No. Amer., 1907, p. 192, pl. 12, fig. 1-3. Egg, Capen, 1S56, pl. 4, figs. 9, 10.

Syleia castanea Wils., Amer. ornith., 1810, vol. 2, p. 97, pl. 14, fig. 4. "Pennsilvania."

Spruce and fir forests in summer, tree tops generally in migration; nests in trees.

ME.- Rare migrant and in northern part a rare summer resident. (May \$) May 14-Aug. Eggs, June S-15.

N. H.- Uneommon migrant and from White Mits. north an uncommon summer resident from 2-4000 ft. May 14-Sept. Eggs, June 3.

VT.- Rare migrant.

Mass.- Usually rare, but sometimes common migrant. May SMay 28 (June 19; July 2, Springfield); Aug. 23-Oet. S.

R. I.-Uncommon migrant. May 9- ; Sept.

Coxs.- Migrant, irregularly rare or common. May 15-30; Sept. 30-Oct. 17.

35.5. Dendroica dominica (Linné) Baird.

\section{Yellow-throated warbler.}

Chapman, Warblers No. Amer., 1907, p. 180, pl. 13, figs. 1, 2.

Motacilla dominica Linné, Syst. nat., ed. 12, 1766, vol. 1, p. 334. "Jamaica, Dominica."

'Tree growth bordering streams or bayous; nests in trees.

Vr.-Recorded from Lunenburg, 1878.

Mass.-Accidental visitor: Dedham, Nor. 4, 1866; Springfield, May, 1892.

Cons.-Aceidental visitor: Connectieut valley, "several"; New Haven. 
PASSERES.

OSCINES.

\section{MNIOTILTIDAE.}

356. Dendroica virens (Gmelim) Baird.

\section{Black-throated green warbler.}

Chapman, Warblers No. Amer., 1907, p. 157, pl. 14, fig. 1-3. Egg, Capen, 18s6, pl. 3, fig. 15.

Motacilla virens Gmel., Syst. nat., 17SS, vol. 1, pt. 2, p. 985. "Pensilvania."

Forest trees, particularly white pines; nests in coniferous trees (once "on the ground," Brewster).

Me.-Common migrant and summer resident. April 2S-Oct. 13. Eggs, May 30-July 1.

N. H.- Common migrant and summer resident mainly below $3000 \mathrm{ft}$. (April 26) May 1-Oct. 25. Eggs, June 2.

VT.-Common migrant and summer resident. May 2-fall.

MAss.-Common migrant and summer resident. (April 20) May 1-Oct. 19 (Nov.3). Eggs, May 30-June 1 .

R. I.-Common migrant and summer resident. (April 25) May 1-Oct. 13. Eggs, June 10.

Cosv.-Common migrant and uncommon local summer resident. (April 27) May 1-23; summer; Sept. 15-Oet. 20. Eggs, May 21-.June 18 .

357. Dendroica Vigorsil (Audubon) Stejneger.

\section{Pine warbler; Pine-creeping warbler.}

Sylvia vigorsii Aud., Omith. biogr., 1832, vol. 1, p. 153, pl. 30. "a salal island of Perioming Creek, . . Pendsilinia."

Sylvicola piuus Lath. Audubon, Birds of Amer., 1S+1, vol. 2, p. 37, pl. 82. Egg, Capen, 18s6, pl. 5, figs. 2, 3.

Coniferous woods, especially pitch-pine groves; nests in trees.

ME.-Common migrant and uncommon local summer resident mainly in southern counties. April 5-Oct. 21. Eggs, June-July 6 .

N. H. - Uncommon local summer resident north to White Mits. April 6-Sept. 30. 
PASSERES.

OSCINES.

MNIOTILTIDAE.

V'T.- Uncommon local summer resident in southern part. April 17 -fall.

Mass. - Common migrant and summer resident; accidental in winter. (Mar. 29) April 1-Nov. 25 (winter). Eggs, May 8-June 16 ; July (2d brood).

R. I.- Common summer resident of pitch-pine groves. April 9-Oct. 29 (Nov. 15). Eggss, May 25.

Coxs.-Common migrant and summer resident. April 14Sept. 29.

35S. Dexirolca palamarum (Gmelin) Baird.

\section{Palm warbler; Redpoll warbler.}

Chapman, Warblers No. Amer., 1907, p. 213, pl. 16, figs. 4, 5.

Motacilla palmanm. Gmel., Syst. nat., 17SS, vol. 1, pt. 2, p. 951. "IN INSULA S. DOMnICl."

Open tree or bushy growth, and edges of fields; nests on the ground.

X. H.- Lncommon fall migrant (a doubtful spring record, Manchester, May 9, 1900). Sept. 7-16.

VT.- Rare fall migrant.

MAss.-Rare fall migriant. Sept. 7-Oct. 2S (Dec. 6, 1902, Belmout).

R. I.-Rare fall migrant: Westerly, Sept. 21, 1596.

Coxs.-Rare fall migrant; once recorded in winter (Jan. 1SSO, at New Haven). Sept. 19-Oct. 10 (Jan.).

359. Dexdrotca paluarum hypochrisea Ridgway.

Yellow palm warbler; Twitch-tail warbler; Yellow redpoll warbler.

Ridgw., Bull. Nuttall ornith. club, 1876, vol. 1, p. 85. "CAnBRIDGE, MLASS."

Sylvicola petechia Lath. Audubon, Birds of Amer., 1S 1 , vol. 2 , p. 55 , pl. 90. 
PASSERES.

OSCINES.

MNIO'TILTIDAE.

Swamps, edges of woods, or open country; nests on or near the ground.

ME.-Common spring and uncommon fall migrant and local summer resident. April 11-Xay 20; summer; Sept. 17-Oct. 20 (Oct. 27, Nov. 2). Eggs, May 27-June 26.

N. H.-Common spring and uncommon fall migrant. April 13Nay 11 ; Sept. 16-Oct. 9.

VT.- Uncommon migrant.

MAss.-Common spring and uncommon fall migrant; said to have wintered. (Mar. 28, 1908, Hyde Park; April 5) April 13May 21; Sept. 5-Oct. 26.

R. I.-Common spring and uncommon fall migrant. April 522 ; Sept. 15-26.

Coxs. - Common spring and uncommon fall migrant. April 9May 5; Oct. 18-Nov. 4.

360. Dendroica discolor (Vieillot) Baird.

\section{Prairie warbler.}

Chapman, Handb. birds east. No. Amer., 1895, p. 364. Egg, Capen, 1886, pl. 5, fig. 1.

Syllia discolor Vieill., Hist. nat. ois. Amér. Sept., 1807, vol. 2, p. 37, pl. 98. "États-Unis et les Grandos îles ANtulles."

Sylcicola discolor (Vieill.). Audubon, Birds of Amer., 18t1, vol. 2, p. 68, pl. 97 .

Dry bush-grown fields and sproutlands; nests in bushes.

N. H.- Rare local summer resident in southern part (Hollis, Manchester).

VT.-Accidental visitor: Uxbridge, Dec. 4, 1884.

MAss. - Uncommon summer resident, mainly near coast; accidental in winter (South Yarmouth, Jan. 2, 1909). May 2-Sept. 25 (Jan. 2). Eggs, May 28-June 18.

R. I.-Common local summer resident. May 4-Sept. (Dec. 4, 1884, Providence). Eggs, May 27-June 14. 
Coxx.- Uneommon summer resident. (April 27) May 1Sept. 15. Eggrs, May 27-June 25.

361. Selurus aurocapildes (Iimné) Swainson.

\section{Oven-bird; Golden-crowned thrush.}

Chalpman, Handb. birds east. No. Amer., 1895, p. 365; Allen, Auk, 1894, vol. 11, pl. 2. Egg, Capen, 18\$6, pl. 5, figs. 4, 5.

Iotacilla aurocapilla Linné, Syst. nat., ed. 12, 1766, vol. 1, p. 334. "in Pensyluania."

Forests and groves; nests on the ground.

ME.-Common migrant and summer resident. (April 23) May 5-Sept. 28. Eggs, May 30-June 10 (July 29).

N. H.-Common migrant and summer resident below $3000 \mathrm{ft}$. May 3-Oct. 2. Egrgs, Jume 2-July 5.

VT.-Common migrant and summer resident. May 5-fall. Eggs, June 7.

MAss.-Common migrant and summer resident. (April 30) May 2-Sept. 27 (Dee. 30, 18s1, Watertown). Eggs, May 17June 16 (July \$).

R. I.-Common migrant and summer resident. May 2Sept. 2S. Eggs, May 19-30.

Cons.-Common migrant and summer resident (April 26) May 3-Sept. Egggs, May 20-July 10.

362. Seicrus noveboracexsis (Gmelin) Bonaparte.

Water-thrush; New York Accentor; Northern water-thrush; Wagtail warbler; Water wagtail.

Ridgway, Bull. 50, U. S. nat. mus., 1902, pt. 2, p. 642. Egg, Capen, 1886, pl. 5 , figs. 6,7 .

Motacilla noveboracensis Gmel., Syst. nat., 17SS, vol. 1, pt. 2, p. 958. "IN Lourisiana, \& Noveboraci sepibus."

Turdus aquaticus Wils. Audubon, Ornith. biogr., 1839, vol. 5, p. 284 , pl. 433, fig. 7 . 
PASSERES

OSCINES.

MNIOTILTIDAE.

Swampy woods and borders of streams; nests on the ground or among tree roots.

Me.-Common migrant and in northern part a less common local summer resident. (April 2S) May 6-Sept. 25. Eggs, May 2S-June 23.

N. H.- Common migrant and less common summer resident, local at lower elevations south of White Mts. (April 30) May 3Sept. 26.

VT.- Common migrant, and mainly in northern part, a less common summer resident.

Mass.-Common migrant and rare local summer resident (near Boston, Lancaster). (April 25) May 2-June 5; summer; Aug. 1-Oct. 16. Eggs, May 21.

R. I.- Common migrant and rare local summer resident (Washington Co.). May 7-(summer); Aug. 12-Sept. 15. Eggs, May 19,20 .

Coxx.-Common migrant. (May 1) May 5-27; Aug.-Oct. 18.

363. Seiurus voveboracexsis notabilis (Ridgway) Ridgway. Grinnell's water-thrush.

Siurus naevius notalilis Ridgw., Proc. U. S. nat. mus., 1S50, vol. 3, p. 12. "Black Hills, Wroming."

Vicinity of streams; nests on or near the ground.

Conv.- Rare migrant: East Haven, Sept. 21, 1904; Hamden, Sept. 7, 1904; Orange, May 20, 1905 (Bishop).

364. Seiurus motacilla (Vieillot) Bonaparte.

\section{Louisiana water-thrush; Large-billed water-thrush.}

Ridgway, Bull. 50, U. S. nat. mus., 1902, pt. 2, p. 639.

Turdus motacilla Vieill., Hist. nat. ois. Amér. Sept., 1S07, vol. 2, p. 9, pl. 65. "Кемтuckт."

Turdus ludovicianus Audubon, Ornith. biogr., 1S31, vol. 1, p. 99, pl. 19. Egg, Capen, 1SS6, pl. 5, fig. S. 
PASSERES.

OSCINES.

\section{MNIOTILTIDAE.}

Wooded borders of streams; nests on the ground or among tree roots.

Mw.-Accidental visitor: Norway, May, 1865 (nec Waterville, 1865).

N. H.- Doubtfully recorded (Dublin).

VT.- Doubtfully" recorded (Brattleboro, "fall").

MAss. - Rare risitor and summer resident (Berkshire Co. and Connecticut ralley). April 13-July 28.

R. I.- Rare summer resident. May 2. Eggs, May 15-28.

Coxx.- Uncommon summer resident in southern part. (Feb. 15, 1852, ? Saybrook) April 13-July. Eggs, Maý 6-June 10.

365. Oporornis lormosa (Wilson) Baird.

\section{Kentucky warbler.}

Chapman, Warblers No. Amer., 1907, p. 235, pl. 1S, figs. 1, 2.

Sylvia formosa Wils., Amer. ornith., 1811, vol. 3, p. S.5, pl. 25, fig. 3. "KentuckT AND Tennessee."

Undergrowth of swampy woods; nests on or near the ground.

VT.-Accidental visitor: Lunenburg, May 30, 1905; also a former doubtful record (Taftsville, May 19, 1884).

Mass.-Accidental visitor: Vellesley, May 14, 1907; also doubtfully recorded from Lynn.

R. I. - Accidental visitor: Newport, spring of 1890.

Coxs. - Rare summer visitor (Lyme; Suffield, Aug. 16, 1876; West Stratford, May 30, 1SSS) and summer resident (Greenwich, with nestlings, July 10, 1892).

366. Ororornis Agilis (Wilson) Baird.

\section{Connecticut warbler.}

Chapman, Warblers No. Amer., 1907, p. 241, pl. 18, figs. 3, 4.

Sylvia agilis Wils., Amer. omith., 1812, vol. 5, p. 64, pl. 39, fig. 4. "ConneCTICU'T." 
PASSERES.

OSCINES.

MNIOTILTIDAE.

'Tamarack swamps, and in migration, damp woods and thickets; nests on the ground.

ME.- Rare fall migrant. Sept. 5-20 (nec Aug. 30, 1878, Cape Elizabeth).

N. H.- Rare fall migrant. Sept. 6-Oct. 11.

TT.-Rare (? spring and) fall migrant. (? Brattleboro, May; ? Rutland, April 24, 1879); Sept. 20-30.

Mass. - Lneommon fall migrant (? aceidental in spring). (May 1890, West Newton, fide Maynard); Sept. 7-Oet. 9.

R. I.- Uneommon fall migrant. Sept. 2t-Oet. 2 (Nor. 12).

Coxx. - Accidental spring and uncommon fall migrant. (Spring, Saybrook; nee New Haven); Sept. 15-Oct. 10.

367. Opororis philadelphia (Wilson) Ridgway.

\section{Mourning warbler.}

Sylvia philadelphia Vils., Amer. omith., 1810, vol. 2, p. 101, pl. 14, fig. 6. "within a Few mles of Philadelphia."

Trichas philadelphia (Wils.). De Kay, Zool. New York, 1844, vol. 2, p. S1, pl. 54, fig. 122. Eggg, Capen, 1886, pl. 5, figs. 12, 13.

Tangles of bushes, rines, and briers; nests on or near the ground.

ME.- Uncommon migrant, and in northern part an uncommon summer resident. (May 16) May 2t-fall. Eggs, June 10-16.

N. H.- Uneommon migrant and from White Mts. north an uneommon summer resident. May 19-Sept. 13. Eggs, June S.

$\mathrm{T}_{\mathrm{T}}$ - Uncommon migrant and summer resident south to Londonderry. May 20-Sept. 20.

Mass. - Rare migrant and in Berkshire Co., a rare summer resident. (Nay 14) May 21-June 13; summer; Sept. 12-30.

R. I.- Rare migrant. May 21-June 5; Sept. 4.

Coxx.- Rare migrant. ILay 12-27; fall. 
PASSERES.

OSCINES.

MNIOTILTIDAE.

368. Geothlypis trichas (Linné) Cabanis.

Maryland yellowthroat; Black-masked ground warbler; Yellowthroat.

Turchs trichas Limné, Sỵst. nat., ed. 12, 1766, vol. 1, p. 293. "in America septentrionali."

Geothlypis trichas brachidactyla (Swains.). Chapman, Warblers No. Amer., 1907, p. 251, pl. 20, fig. 3-5. Egg, Capen, 1S86, pl. 5, fig. 9-11.

Weed patches and thickets near water; nests on or near the ground.

Me.-Common migrant and summer resident. May 6-Oct. 13 (Oct. 22). Eggs, May 28-June 12.

N. H.- Common migrant and summer resident at lower elevations. Maỵ 6-Oct. 7. Eggs, June 3-21.

VT.-Common migrant and summer resident. Eggs, June 2.

MAss. - Common migrant and summer resident; aecidental in winter. (April 30) May 2-Oct. 21 (winter). Eggs, May 24June 17.

R. I.-Common migrant and summer resident. (April 22)-Oct. 17. Eggs, May 25-June 5.

Coxs-Common migrant and summer resident. (April 29) May 4-Nor. 1. Eggs, May 22-June 18.

369. ICterta virens (Linné) Baird.

Yellow-breasted chat; Ghost-bird (Indian); Yellow chat.

Audubon, Birds of Amer., 1842, vol. 4, p. 162, pl. 244. Egg, Capen, 1886, pl. 5, figs. 14, 15 .

Turdus virens Linné, Sỵst. nat., ed. 10, 1758, vol. 1, p. 171. "Anerica."

Bushy thickets; nests in low bushes.

Me.-Accidental visitor (five instances). June 6-Oet. 5. 
PASSERES.

OSCINES.

\section{MNIOTILTIDAE.}

N. H.- Doubtfully recorded.

TT.- Very rare summer resident in extreme south: Powmall, June 16, 1896.

Miss. - Rare local summer resident near coast and in western valleys. May 10-Sept. 10 (Oct. 26, 1907, Lynnfield). Eggs, May 1S-June 18.

R. I.-Locally common summer resident in southern part. May 3-Aug. 5. Eggs, May 31-June 13.

Conn.- Locally common summer resident. May 5-Aug. Eggs, May 22-July 7.

370. Wilsonia citrina (Boddaert) A. O. U. comm.

\section{Hooded warbler.}

Muscicapa citrina Bodd., Tabl. d. planch. enlum. d'hist. nat., 17S3, p. 41. Based on Buffon.

Wilsonia mitrata (Gmel.). Chapman, Warblers No. Amer., 1907, p. 269, pl. 21, fig. 1-3. Egg, Capen, 18s6, pl. 6, fig. 1.

Undergrowth of woods; rests in bushes.

Me.-Accidental visitor: Falmoutlr, Sept. 10, 1904.

YT.-Doubtfully recorded.

Mass.- Rare visitor; possibly breeding in soutluwestern valleys. May S-Oct. 15 (June 25, 1879, Brookline; June 25, 1888, Provincetown).

R. I.-Accidental visitor: Kingston.

Conx.- Uncommon summer resident of laurel undergrowth. May 2-Sept. Eggs, May 25-June 24.

371. Wilsonia pusilla (Wilson) Bonaparte.

Wilson's warbler; Black-capped flycatching warbler; Wilson's blackcap.

Chapman, Warblers No. Amer., 1907, p. 274, pl. 22, figs. 1, 2. Egg, Capen, 18s6, pl. 6, fig. 2. 


\section{MNIOTILTIDAE.}

Muscicapa pusilla Wils., Amer. ornith., 1811, vol. 3, p. 103, pl. 26, fig. 4. "solthern States, aND.... NeW Jersey and DELAWARE."

Bushy growth near streams or in swamps; nests on the ground.

ME.-Uncommon migrant and summer resident (south to Pittsfield). May 7-June 5; summer; Aug. 18-Sept. 11 (Oct.). Eggos, May 31-.June 19.

N.H.- Uncommon migrant and north of White Mts. a rare local summer resident (Lancaster). May 9-29; (summer); Aug. 15-Oct. 3. Eggs, June 6.

VT.-Uncommon migrant; (?summer). May 17; Aug. 24.

Mass. - Uncommon migrant. May 7-30 (June 1); Aug. 23Sept. 27 (Nov. 20, 1898, Belmont).

R. I.-Uncommon migrant. May 15-22; Sept. (Nor. 30).

Coxw. Uncommon migrant. May 10-25; Sept. 27.

372. Wilsonia Canadensis (Limné) Coues.

\section{Canadian warbler; Canada flycatcher.}

Chapman, Warblers No. Amer., 1907, p. 280, pl 22, fig. 3-5. Egg, Capen, 18s6, pl. 6, figs. 3, 4.

Muscicapa canadensis Linné, Sỵst. nat., ed. 12, 1766, vol. 1, p. 327. "CaNada."

Wet woods or thickets along streams; nests on or near the ground.

Me.-Common migrant and summer resident. (April 25, Bangor; May 7) May 10-Sept. 12. Eggs, May 30.

N. H.-Common migrant and less common local summer resident below $3000 \mathrm{ft}$. May 13-Sept. 13. Eggs, June 9-13.

VT.-Conmon migrant and less common local summer resident. Nay 15 -fall.

MAss. - Common migrant and less common summer resident mainly in western part. May 9-Sept. 29. Eggs, June 9-26.

R. I.- Common migrant and uncommon local summer resident. May 11-fall. 
PASSERES.

OSCINES.

MNIOTILTIDAE.

Coxx.- Common migrant and rare local summer resident (Portland). (May 6) May 12-fall. Figgs, May 30.

373. Setophaga ruticilla (Limné) Swainson.

\section{American redstart.}

Chapman, Warblers No. Amer., 1907, p. 287, pl. 23, fig. 1-3. Egg, Capen, 1Ss6, pl. 6, fig. 5 .

Motacilla ruticilla Linné, Syst. nat., ed. 10, 1758, vol. 1, p. 186. "Anerica."

Orchards, shade and forest trees; nests in trees.

ME.-Common migrant and summer resident. May 4-Oct. 1 (Oct. 13). Egggs, June 2-30.

N. H.-Common migrant and summer resident below $3000 \mathrm{ft}$. May 4-Oct. 5. Eggs, May 30-June 13.

VT.-Common migrant and summer resident. Nay S-fall.

MAss.-Common migrant and summer resident. (April 13, 23, 30) May 4-Oct. 12 (Nov. 1, 1908, Annold arboretum). Eggs, May 29-June 21.

R. I.- Common migrant and summer resident. May 1-Sept. S. Eggs, June 1-13.

Coxv-C Common migrant and summer resident. May 3Oct. 16 (Oct. 26). Eggs, May 20-June 27.

\section{MOTACILLIDAE.}

37. Anthus rubescens (Tunstall) A. O. U. comm.

\section{American pipit; Brown lark; Titlark; Wagtail.}

Alauda rubescens 'Tunstall, Ornith. Britannica, 1771, p. 2. No locality; based on Red lark from London and North America (fide Oberholser).

Anthus pensilvanicus (Lath.). Ridgway, Butl. 50, U. S. nat. mus., 1904, pt. 3, p. 12.

Anthus pipiens Audubon, Ornith. biogr., 1S32, vol. 1, pl. $\$ 0$. 
PASSERES.

OSCINES.

MOTACILLIDAE.

Barren grounds, fields and sea coasts; nests on the ground.

ME.-- Rare spring and common fall migrant. May 2-1S (Jume 1, 1907, Lubec); Sept. 13-Oct. 25 (Dec. 13, 1901, Seguin Id.).

N. H.- Rare spring and common fall migrant. April 3-May 10; Sept. 15-Nor.

VT.- Rare spring and uncommon fall migrant.

MAss.- Uncommon spring and common fall migrant; accidental in winter (Boston Hiubor, Feb. 25, 18s2; Newburyport, Jan. 4, 1S7S). April 1S-May 20 (Jume S); (Aug. 29, 1S8S, Monomoy Id.) Sept. 10-Nov. 24 (winter).

R. I.- Rare spring and common fall migrant. Mar. 29-May 15; Sept. 16-Nov. 28.

Coxs.-Rare spring and common fall migrant. April-Mayy; Sept. 15-Nor. 14 (Dec. 25, 1908, South Norwalk).

\section{MIMIDAE.}

375. Minus polyglottos (Limné) Bonaparte.

\section{Mockingbird.}

Chapman, Handb. birds east. No. Amer., 1895, p. 377; Birdlife, 1898, pl. 66. Egg, Capèn, 18\$6, pl. 1, fig. S-10.

Turdus polyglottos Limné, Syst. mat., ed. 10, 175S, vol. 1, p. 169. "Virginia."

Thickets and dense tree clumps; nests in bushes or trees.

ME.-Occasional visitor at all seasons or escape; said to have bred at Leeds about 1885 .

N. H.- Accidental risitor or escape: Hampton; Milford.

VT.-Accidental visitor or escape; pair nested at Lunenburg, 1 SSt.

MAss.- Occasional visitor at all scasons or escape; rare summer resident (Arlington, Groton, Marshfield, ? Provincetown, Springfield). Eggs, Junc 13-20.

R. I.-Occasional visitor at all seasons (or escape). 
PASSERES.

OSCINES.

MIIIIDAE.

Conx- Rare summer resident (more frequent formerly); occasional visitor (or escape) at all seasons. Eggs, June 20 (Jewett City).

376. Dumetella carolinexsis (Linné) A. O. U. comm.

\section{Catbird; Pussy.}

Muscicapa carolinensis Limné, Syst. nat., ed. 12, 1766, vol. 1, p. 32S. "CAROLINa."

Orphens carolinensis (Linné). Audubon, Birds of Amer., 1S41, rol. 2, p. 195, pl. 140. Egg, Capen, 1.8s6, pl. 1, fig. 11.

Bushy thickets near water; nests in bushes or among vines.

ME.- Common summer resident, mainly of southern counties. May 17-Oct. 4. Eggs, June 2-17; July (2d brood).

N. H.- Common summer resident north to White Mt. vallers; accidental in early winter (Concord, Dec. 3, 1901; Wilton, Dec. 25, 1904). May 6-Oet. 14 (Dec. 25). Eggs, June 6-July 2S (? 2d brood).

VT.-Common summer resident.

Mass.-Common summer resident; accidental in winter. (April 13, 1908, Westwood) April 29-Oct. 16 (Dec.; winter 190S09, Forest Hills). Egugs, May 22-.Tune 20.

R. I.-Common summer and casual winter resident. (April 24)-Oct. 13 (Dec. 28-Mar. 4, Mar. 19). Eggs, May 25-June 10.

Conx-Common summer resident. May 3-Nov. 4. Eggs, May 17-23; July 26 (2d brood).

377. Toxostoma Rufur (Limné) Cabanis.

Brown thrasher; Brown thrush; Mavis; Mocking thrush; Planting-bird; Song thrush; Thrasher.

Turdus rufus Linné, Syst. nat., ed. 10, 1758, vol. 1, p. 169. "IN AMERICA septextrional \& Meridonati."

Orpheus rufus (Limné). Audubon. Birds of Amer., 1St1, vol. 3, p. 9, pl. 141. Eggr, Capen, 1s\$6, pl. 1, figs. 12, 13. 
PASSERES.

OSCINES.

IIIIIDAE.

Thickets and sprontlands; nests in bushes or rarely on the ground.

Me.-Common summer resident in southwestern counties. (? April S) April 2S-Oet. 14.

N. H.-- Uncommon summer resident north to White MIt. valleys. (April 16)-Oct. 10.

Tr.- Uncommon summer resident at lower elevations.

MAss.-Common summer and aceidental winter resident (Arlington). (April 16) April 20-Oct. 26 (Nov. 4; winter). Eggs, May 10-June 21.

R. I.-Common summer resident; easual in winter. (April 14)-Oct. 13 (Jan. 30). Eggs, May 19-June 4.

Coxi-Common summer resident. April 22-Oct. 2.

\section{TROGLODYTIDAE.}

37s. 'ThrYothorus ludovicianus (Latham) Bonaparte.

\section{Carolina wren; Great Carolina wren; Mocking wren.}

Sylvia ludoviciana Lath., Index omith., 1790, vol. 2, p. 54 S. "Louisiana."

Troglodytes ludovicianus Bonap. Audubon, Birds of Amer., 1St1, vol. 2, p. 116, pl. 117. Egg, Capen, 1SS6, pl. 2, fig. 11 .

Dense undergrowth and tangles; nests in cavities of trees or erevices of buildings.

Me.-Accidental visitor (Falmouth, Oct. 3, 1908; Waterville) and accidental summer resident (Norway Lake, eggs, June, 1SS3, fide Knight).

N. H.-Accidental visitor: Alstead, July 6, 1903; Rye Beach, Aug. 7, 1880; Webster, about 1900.

I'T.-Doubtfully recorded.

Mass.- Occasional visitor at all seasons and very rare summer resident (Naushon Id., July, 1901). 
PASSERES.

OSCINES.

TROGLODYTIDAE.

R. I.- Occasional visitor in summer and fall, and rare summer resident (at Middletown). April 9-Nor. 28. Eggs, April 15.

Covx.- Occasional visitor at all seasons and rare resident (near Bridgeport, Chester, South Norwalk). Eggs, July 16.

379. Thromanes Bewickil (Andubon) Ridgway.

\section{Bewick's wren.}

Ridgway, Bull. 50, U. S. nat. mus., 1904, pt. 3, p. 552.

Troglodytes bewickii Aud., Ornith. biogr., 1831, vol. 1, p. 96, pl. 1S. "about five miles from St. Francisville, . . LoulsIANA."

Brush piles, orehards, and vicinity of outbuilelings; nests in carities of trees or buildings.

Me.-Doubtfully recorded (Seguin Id., Oct. 6).

N. H.-Aceidental risitor: Alton, April 25, 1890.

380. Troglodytes aedon Vieillot.

\section{House wren.}

Vieill., Hist. nat. ois. Amér. Sept., 1807, vol. 2, p. 52, pl. 107. "Canada,... Nouvelle-Écosse." Audubon, Birds of Amer., 1841, vol. 2, p. 125, pl. 120. Egg, Capen, 1SS6, pl. 2, figs. 12, 13.

Brush piles, orchards, and rieinity of outbuildings; nests in hollow limbs, bird boxes, or any convenient crevice.

Me.- Uncommon local summer resident (to Caribou). May S-Aug.

N. H.- Uncommon summer resident becoming rare among White MIt. vallers. May 1S-Sept. 24.

VT.-- Uncommon summer resident. Nay 11-fall.

MAss.- Locally common summer resident. (April 24) May 1Oct. 30. Eggs, May 25-June 5.

R. I.- Locally common summer resident; formerly more common. April 26-Sept. Eggs, May 21-27.

Cons.-Common summer resident. April 25-Oct. 30. 
381. Naxues hiemalis (Vieillot) A. O. U. comm.

\section{Winter wren.}

Troglodytes hiemalis Vieill., Nouv. dict. d'hist. nat., 1819, vol. 34, p. 514. "Nouvelle-Écosse et dans l'État de New York." Audubon, Birds of Amer., 1St1, rol. 2, p. 12S, pl. 121. Egg, Capen, 1S86, pl. 2, fig. 14.

Damp woods or thickets near water, and in migration, tangles, brush heaps, and brier patches; nests in crevices of upturned roots.

Me.- Common migrant and in northern part a common summer resident; very rare winter resident (Bangor). (April 1) April 9-Nov. 17 (winter). Eggs, May 20-June 3; Aug. S (2d brood).

N. H.- Common migrant and less common summer resident, local south of White Mts.; rare winter resident in southeast part. April 5-Nor. 15 (winter). Eggs, May 20-July 23 (2d brood).

$V_{\mathrm{T}}$ - Uncommon migrant, less common summer resident in mountainous parts south to Londonderry; may winter. April 7Nov. 24 (Dec. 30, Jau. 1). Eggs, June 4.

Mass.- Uncommon migrant, less common summer resident (Berkshire Co., and locally east, Lynn, Wiuchendon); rare winter resident near coast. April 4-29; summer; Sept. 2-Nor. 25; winter.

R. I.- Uncommon migrant and rare winter resident; rare local summer resident (Washington Co., nest with young, May 24, 1908). April; (summer); Sept.-Nov. 2S; winter.

Coxs.- Uncommon migrant and less common winter resident. Oct. 2-April.

3S2. Cistothorus stellaris (Lichtenstein) Cabanis.

\section{Short-billed marsh wren.}

Troglodytes stellaris Licht., in Naumann's Vögel Deutschl., 1823, vol. 3, tab. ad p. 724. "CAROLINA." 
PASSERES.

OSCINES.

TROGLODYTIDAE.

Troglodytes brevirostris Nutt. Audubon, Birds of Amer., 1S41, rol. 2, p. 13s, pl. 124. Egg, Capen, 1Ss6, pl. 3, fig. 1.

Wet grassy meadows; nest usually built among tall grass stems near the gromind.

ME.- Rare local summer resident (Bangor, Glenburn). May 4-Oct. 10 .

N. H. - Rare loeal summer resident in southern part; rare visitor in White Mt. region.

Mass.- Tery local summer resident, sometimes common. May 6-Oct. 9. Eggs, May 25-June 1S; July 7 (? 2d brood).

R. I.- Rare local summer resident (Newport). Eggs, June 6.

Coxx.- Rare local summer resident. Spring-Sept. 17.

383. Telahodytes palustris (Milson) Henry.

\section{Long-billed marsh wren.}

Ridgway, Bull. 50, U. S. nat. mus., 1904, pt. 3, p. 4\$9. Egg, Capen, 1886, pl. 2, fig. 15 .

Certhia palustris Wils., Amer. ornith., 1S10, vol. 2, p. 58, pl. 12, fig. 4. "Penssyliania... Along the tide WATER OF oUR RIVERS."

Cat-tail swamps; nests among the flags.

VT.- Rare visitor: Brattleboro, May, 1902; Middlebury; Rutland, May 27, 1907.

Mass. - Common local summer resident in western vallers and coastwise (Essex Co.); rare winter resident (Fresh Pond). (May 2) May 15-Nov. 30 (winter). Eggs, June 5-29 (Aug. 1).

R. I.-Common local summer resident. May-Oct. 6. Eggs, May 31-June 16.

Cosx.-Common summer and rare winter resident. May 5(winter). Eggs, June 7-July $2 S$ 
PASSERES.

OSCINES.

CERTHIIDAE.

384. Certhia famliaris americana (Bonaparte) Ridgway.

\section{Brown creeper; Tree creeper.}

Certhia americana Bonap., Geogr. and comparative list birds Europe and No. Amer., 183S, p. 11. "Western AND Northern Parts" of No. Anerica.

Certhia familiaris Linné. Audubon, Birds of Amer., 1841, p. 109, pl. 115. Egg, Capen, 1S\$6, pl. 2, fig. 10.

Cool mixed or eoniferous woods in summer, tree growth generally at other seasons; nests behind loose flake of bark.

Me.- Common migrant, less eommon summer and winter resident. Eggs, May 31-June 23.

N. H.- Resident, breeding locally south of White MIs., and more eommonly north. Eggs, May.

$V_{\mathrm{T}}$.-Common migrant and winter resident; uncommon local summer resident.

Mass. - Common migrant and winter resident; less common summer resident in western part, but rare and local in eastern swamps (to Taunton). Sept. 12-May \&; summer. Eggs, May 6-23.

R. I.-Common migrant and winter resident. Sept. 2-April 26.

Coxs.-Common migrant and winter resident; rare summer resident. Late Sept.-late April (summer).

\section{SITTIDAE.}

385. Sitta carolinensis Latham.

\section{White-breasted nuthatch; Sapsucker.}

Lath., Index ornith., 1790, vol. 1, p. 262. " "IN AliericA, JAliAiCA; Europaea minor." Audubon, Birds of Amer., 1S42, vol. 4, p. 175, pl. 247. Egg, Capen, 1S86, pl. 2, fig. S.

Orchards, shade and forest trees; nests in holes in trees or stumps. 
PASSERES.

OSCINES.

SITTIDAE.

Me., N. H., V'T.-Common migrant, uneommon summer and winter resident. Eggs, May 1-15 (МЕ.).

MAss.-Common migrant and winter resident; uneommon summer resident. Eggs, April 3-May 1.

R. I.-Common migrant and winter resident; uncommon summer resident. Sept. 14-April 22; summer. Eggs, April 20May 22.

Coxx-Common migrant and winter resident, less common summer resident. Eggs, May S-June 7.

386. Sitta Canadexsis Linné.

Red-breasted nuthatch; Canada nuthatch; Red-bellied nuthatch.

Linné, Syrst. nat., ed. 12, 1766, rol. 1, p. 177. "CAnada." Audubon, Birds of Amer., 1842, vol. 4, p. 179, pl. 248. Egg, Capen, 1856, pl. 2, fig. 9 .

Coniferous woods, or, on migrations, tree growth generally; nests in holes exeavated in dead trees.

Me.-Common resident south to Cape Elizabeth; elsewhere a periodically common winter resident. Eggs, May 6-June 2 (early July).

N. H.- Common resident from White Mts. north, elsewhere a rare summer and irregular winter resident. Eggs, May 20.

VT.- Uncommon winter visitor and local summer resident. Eggs, May 5-30.

MASs.- Irregular fall and winter visitor; in western part at higher levels an uncommon, and elsewhere a rare and irregular summer resident. Aug. 15-May 20; summer. Egggs, Nay 1SJune 10.

R. I.- Uncommon and irregular fall and winter visitor. Oct. 5 -winter.

Coxx.-- Uncommon and irregular fall and winter risitor. (Aug. 28) Sept. 24-April 19. 
357. B.teolopies bicolor (Limné) Cabanis.

\section{Tufted titmouse.}

Parus bicolor Linné, Sịst. mat., ed. 12, 1766, vol. 1, p. 340. "Ix America septentrionali." Audubon, Birds of Amer., 1S+1, vol. 2, p. 143, pl. 125.

Woodlands generally; nests in holes of trees and stumps.

ME.-Accidental visitor: near Orono.

N. H., VT.-Doubtfully recorded.

Coxs.- Rare visitor: near Hartford; Lyme, Feb. 27, 1S72, Jan. 1874; New Haven (Linsley).

3SS. Pexthestes atricapillus (Limné) Ridgray.

\section{Chickadee; Black-capped chickadee or titmouse.}

Parus atricapillus Limné, Sỵst. nat., ed. 12, 1766, rol. 1, p. 341. "CANadA." Audubon, Birds of Amer., 1St1, rol. 2, p. 146, pl. 126. Eggg, Capen, 1Ss6, pl. 2, figs. 4, 5.

Tree growth generally; nests near water in low dead stumps.

Me.-Common resident. Eggs, May 10-June $1 S$.

N. H.-Common resident, breeding below $3000 \mathrm{ft}$.

VT.-Common resident. Eggs, May 10.

Miss.-Common winter and less common summer resident. Eggs, May 4-June 25.

R. I.-Common winter and less common summer resident. Egges, May 9-18.

Coxx.- Common resident. Eggs, May 10-June 4.

3S9. Pexthestes hudsoxicts (Forster) Ridgway.

\section{Hudsonian chickadee.}

Parus hudsonicus Forst., Phil. trans. roy. soc. London, 1772, vol. 62, pp. 40S, 430. "Severi River.... Ad Sindu Hudsonis." Audubon, Birds of Amer., 1S41, rol. 2, p. 155, pl. 12S. Egg, Capen, 18s6, pl. 2, figs. 6, 7 . 
PASSERES.

OSCINES.

PARIDAE.

Spruce and fir forests; nests in holes in low trees or stumps.

ME.- Rare resident in northern and eastern counties; elsewhere an uncommon or rare winter visitor.

N. H. - Uncommon resident mainly above $3000 \mathrm{ft}$. on White Mts., north; elsewhere a rare winter visitor.

$V_{T}$.- Uncommon resident in northern part and on higher mountains (MIt. Stratton, Sutton); elsewhere a rare winter visitor.

Mass. - Rare late fall and winter visitor; once in Plymouth Co., June 5, 1896. Oct. 1S-April 5 (June 5).

R. I.-Aecidental late fall visitor: Smithfield, Nor. 1, 1880.

Coxx.-Accidental late fall visitor: New Haven, Nov. 13, 1875.

\section{SYLVIIDAE.}

390. Regulus Satrapa Lichtenstein.

\section{Golden-crowned kinglet; Golden-crested wren.}

Licht., Terzeichn. d. doubl. d. Mus. Berlin, 1S23, p. 35. "Air[erica] sertentrionali]." Audubon, Birds of Amer., 1S41, vol. 2, p. 165, pl. 132. Egg, Capen, 1SS6, pl. 2, figs. 1, 2.

Spruce and fir forests in summer, tree growth generally at other times; nests in conifers.

Me.-Common migrant and less common winter resident; common summer resident in northern part. Eggs, June 15-24. . N. H.-Common migrant, less common winter resident; common summer resident from White Mts. north, locil to the south. Egges, May 24.

YT-Common migrant and winter resident; less common local summer resident. Sept. 18-May 7; summer.

MAss.-Common migrant and winter resident; uncommon summer resident in western part but rare and local in eastern (Lrinn, Plymouth Co., Winchendon). Sept.-April; summer. Eggs, MayJune 29.

R. I.-Common migrant and winter resident. Sept. 25-April 2. 
PASSERES.

OSCINES.

SYLVIIDAE.

Coxx-Common migrant and winter resident. Oet. 13-May.

391. Regulus calexdula (Linné) Lichtenstein.

\section{Ruby-crowned kinglet; Ruby-crested wren.}

Audubon, Birds of Amer., 1841, vol. 2, p. 168, pl. 133.

Motacilla calendula Limné, Sỵst. nat., ed. 12, 1766, vol. 1, p. 337. "Pexistluania."

Spruce and fir forests, and in migrations, tree growth generally; nests in conifers.

Mr.-Common migrant and probably a rare summer resident in northern part (Ft. Kent, with young; near Bangor, nest without eggs). April 14-May 16 (May 23); (summer); Sept. 25-Oct. 22.

N. H.- Common migrant. April 14-Nay 11; Sept. 9-Oct. 20.

VT.-Common migrant. April 11; fall.

MAss.-Common migrant; casual in winter (at Highland Light, Jan. 15, 1892; Fresh Pond, Cambridge, Dec. 23, 1899). April 5May 13; Sept. 23-Nor. 20 (winter).

R. I.-Common migrant. April-May 6; Oet.

Conv-Common migrant. April 11-(May 16); Oct. 13-Nor. 24.

392. Polioptila caerulea (Linné) Sclater.

\section{Blue-gray gnatcatcher.}

Motacilla carrulea Limné, Surst. nat., ed. 12, 1766, vol. 1, p. 337. "Pexsmluania."

Culicivora coerulea Lath. Audubon, Birds of Amer., 1840, rol. 1, p. 24t, pl. 70. Egg, Capen, 1Ss6, pl. 2, fig. 3.

Woodlands; nests in trees.

Me.-Accidental risitor: Cape Elizabeth, Aug. 29, 18s0; April $18,1896$.

N. H., IT.-Doubtfully recorded.

MAss.-Accidental risitor (? may once have bred near Taunton). Aug. 27-Dec. 1S. 
PASSERES.

OSCINES.

SILVIIDAE.

R. I.-Accidental visitor (several instances). May 23, June 24. Conv- - Rare summer risitor (five instances, nec Waureganl). April 6-Sept. 1.

\section{TURDIDAE.}

393. Hrolocichla mustelixa (Gmelin) Ridgway.

Wood thrush; Mountain thrush (Vt.); Swamp angel; Swamp thrush; Wood robin.

Turdus mustelinus Gmel., Syst. nat., 178S, vol. 1, pt. 2, p. 817. "ix Noveboraco." Seebohm and Sharpe, Monogr. of Turdidae, 1898, pt. 4, pl. 43. Chapman, Handb. birds east. No. Amer., 1895, p. 395, fig. Egg, Capen, 1886, pl. 1, fig. 2.

Bushy growth near edges of moist shady woods; nests in bushes or trees.

ME.- Rare summer resident in southwestern part (to Franklin Co.). Spring-Sept. 6.

N. H.- Rare summer resident north to White MIt. valleys. May 15 -Sept.

VT.- Uncommon summer resident at lower elevations (to Mit. Mansfield and Willoughby Lake). May 10-fall.

MAss. - Common summer resident, mainly at low elerations. (April 26) May 1-Sept. 28 (Oct. 10; Nov. 18, 1897, Wellesley Hills). Eggs, May 25-June 15 (June 26).

R. I.-Common summer resident. May 4-Sept. Eggs, May 21-June 6.

Corx.-Common summer resident. May 1-Oet. Eggrs, May 27-June 21.

394. Hriocichla fuscescens (Stephens) Ridgway.

Wilson's thrush; Cheeury; Nightingale; Swamp robin; Tawny thrush; Veery.

Turdus fuscescens Steph., Shaw's Gen. zool., 1817, vol. 10, pt. 
PASSERES.

OSCINES.

TURDIDAE.

1, p. 182. "to the vorth of Pexsyluacia." Seebohm and Sharpe, Monogr. 'Turdidae, 1S9S, pt. 4, pl. 44. Egg, Capen, 1856, pl. 1 , fig. 7 .

Sramps and undergrowth along streams; nests on or near the ground, rarely in trees.

Me.-Common summer resident. (? April 17, 26) May 1Sept. 20 (Oct. 1). Eggs, June 1-1S (July 1).

N. H.-Common summer resident below $1500 \mathrm{ft}$. May 10Sept. Eggs, June 9.

VT.-Common summer resident. May 11-Sept. Eggs, May 30-June 28 (July 26).

MLass.-Common summer resident. (April 30) May 1-Sept. 27 (Oct. 14, 1S71, Fresh Pond, Cambridge). Eggs, May 20-June 30 .

R. I.- Common summer resident. (April 29)-Sept. 25. Eggs, May 26-31.

Coxx.-Common summer resident. May 1-Sept. 1.

395. Hrlocichla fuscescens salicicola Ridgray.

Willow thrush; Newfoundland thrush.

Ridgw., Proc. U. S. nat. mus., 1S\$2, vol. 4, p. 374. "Rocky Mountain region.... Fort Garland, Colo.," etc.

Swamps and undergrowth bordering streams; nests on or near the ground.

MAss.- Rare migrant: Lanesboro, Sept. 27, 1900; Lowell (these are the race "fuliginosa" Howe, considered by the A. O. U. committee inseparable from salicicola and by Ridgway as synonymous with $H$. fuscescens).

R. I.- Rare migrant: Bristol, Sept. 24, 1S99; Nerrport, Sept. 25,1855 .

Coxn.-Rare migrant. May 5, 6, 14, 16; Sept. 23 (= salicicola, Bishop). 
PASSERES.

OSCINES.

TURDIDAE.

396. Hriocichla Aliciae (Baird) Ridgway.

Gray-cheeked thrush; Alice's thrush; Arctic thrush.

Turdus aliciae Baird, C. S. war dept., expl. and surv. Miss. Riv. to Pac. Ocean, 185S, pt. 2, birds, p. 217. "Illurnors, . . . LPper Missourr." Seebohm and Sharpe, Monogr. Turdidae, 1898, pt. 4, pl. 45 , fig. 1 .

Dense fir thickets in summer, moist shaded spots on migration; nests in low trees.

Me.- Uncommon migrant. May 20-25; Sept. 20.

N. H.- Rare migrant: Dublin, Oct. 2, 1899.

Y'T.-Rare migrant. May $1 S$.

Mass.- Uncommon migrant. (May 13) May 17-June 3 (June 1S); Sept. 10-Oct. 9.

R. I.-Uncommon migrant. May 20; Sept. 23-Oct. 7.

Coxs.- Uncommon migrant.

397. Hylocichla aliciae bickenelu Ridgway.

\section{Bicknell's thrush.}

Ridgw., Proc. L. S. nat. mus., 1SS2, vol. 4, p. 377. "Slide Mountain, Ulster County, New York." Bull. 50, L. S. nat. mus., 1907, pt. 4, p. 62. (Unfigured).

Fir and spruce thickets in summer, moist shady spots on migration; nests in low trees.

Ne.-Common local summer resident on higher mountain tops (Mt. Abraham, Ktaadn, ete.); Maine breeding birds may be true aliciae (Knight). May-Sept. 20.

N. H.- Common summer resident above $3000 \mathrm{ft}$. to tree limit among White Mis., south to Sandwich Range. May 25-Sept. 15. Eggs, June 22.

VT.- Uncommon summer resident on higher monntains: Jay Peak, Mt. Annanance, Mt. Killington, Mt. Mansficld, Pico Peak, etc. May-Sept. Eggos, June 22.

MAss. - Lncommon migrant, and on summit of Mt. Greylock 


\section{TURDIDAE.}

(3505 ft.) a rare summer resident. (Maly 2)-May 30; summer; Sept. $1 \mathrm{~S}$-Oct. 25.

R. 1.- Uncommon migrant. Oct. 9.

Coxs.- Uncommon migrant.

39S. Hrlocichla ustulata swainsonil (Cabanis) Ridgway.

Olive-backed thrush; Flycatching thrush; Mosquito thrush; Swainson's thrush; Swamp robin.

Turdus suainsonii Cab., Fauna Peruana, 1S45-46, p. $18 S$. "Peru"; specimen described from New Jerser (= type locality). Seebohm and Sharpe, Monogr. 'Turdidae, 1s9s, pt. 4, pl. 45, fig. 3.

Turdus ustulatus swainsonii (Cab.). Chapman, Handb. birds east. No. Amer., 1595, p. 39S. Egg, Capen, 1S\$6, pl. 1, figs. 5, 6.

Cool shady thickets; nests in low trees or bushes.

ME.- Common migrant and in northern part a common summer resident. (May 6) May 9-26; summer-Oct. 23. Eggs, May 29-June 9 (early July).

N. H.-Common migrant, and from White MIts. north a common summer resident, local to the south. May 16-Oct. Eggs, June 16-July 11.

VT.-Common migrant and less common summer resident mainly in northern part and at higher levels south to Londonderry.

Mass.- Common migrant and in higher parts of Berkshire Co., an uncommon summer resident. May 3-June 7 ; summer; Sept. 9-Oct. 21.

R. I.- Common migrant. May S-; Sept. 27-Oct. 2.

Conx.-Common migrant. May 10-26; Sept. 4-Oct. 13.

399. Hylocicila guttata Pallasio (Cabanis) Fason and Hoffmann.

Hermit thrush; Ground swamp robin (Me.); Rufous-tailed thrush; Solitary thrush; Swamp robin or thrush; Woodchuck.

Turdus pallasii Cab., Arch. t. naturgesch., 1847, vol. 13, pt. 1, 
PASSERES.

OSCINES.

TURDIDAE.

p. 205. Lorality not given; based on Cab., in Tschudi's Fauna Peruana. Scebohm and Sharpe, Monogr. 'Turdidae, 189S, pt. 4, pl. 46 , fig. 2 .

Turdus aonalaschliae pallasii (Cabb.). Chapman, Handb. birds east. No. Amer., 1S95, p. 400. Egg, Capen, 1S\$6, pl. 1, figs. 3, 4.

Undergrowth of dry woods; nests on the ground.

Me.-Common migrant and summer resident; may winter rarely. (April 5) April 13-Nor. 26 (Dec. 25, 1904, Cape Elizabeth). Eggs, (May 1) May 17-June 10; July 14-Aug. 10 (2d brood).

N. H.- Common migrant and summer resident below $3000 \mathrm{ft}$. (April 5) April 10-Nor. 2t. Egges, -July S.

VT.-Common migrant and summer resident. (April 4) April 10-fall.

MAss.-Common migrant, less common summer resident in western, local in eastern part (Cape Cod, Martha's Vineyard); rare winter resident near coast. (April 1) April S-Nor. 30 (winter). Eggs, June 14.

R. I.--Common migrant and rare winter resident; rare local summer resident (Kent Co.). April 10-(summer); Oct.-Nor. 20 (Dec. 3, winter). Eggs, June 6 .

Coxx-Common migrant, uncommon local summer resident (Bear Mt., Hartford Co., Norfolk, Salisbury); rare winter resident. (April 3) April 12-May; summer; Oet-Nov. 20 (winter).

400. Planesticus migratorius (Linné) Coues.

\section{American robin.}

Turdus migratorius Limné, Syst. nat., ed. 12, 1766, vol. 1, p. 292. "in Averica septextrionali." Audubon, Birds of Amer., 1St1, vol. 3, p. 14, pl. 142. Eggg, Capen, 1S\$6, pl. 1, fig. 1.

Ubiquitous, but a roiding woods in breeding season; nests in trees, on ledges of buildings, or on the ground.

ME., N. H., VT.-Common nigrant and summer resident; rare winter resident mainly in southern part. Mar. 3-Nov.; win- 
TURDIDAE.

ter. Eggs, (Me.) April 28-June 15; July-early Aug. (2d and 3d broods).

Mass.-Common migrant and summer resident; less common winter resident chiefly near coast. Mar. S-Nov. 23; winter. Eggs, April 25-July 25 (2d brood) (! Oct. 29, 1903, Brandon).

R. I.-Common migrant, less common summer and winter resident. Mar. 11-Nov. 28; winter. Eggs, April 17-June 7; July.

Coxs.- Common migrant and summer resident; less common winter resident. Feb. 18-Nov.; winter. Eggs, April 27-July 2. 401. Ixoreus naevius (Gmelin) Richmond.

\section{Varied thrush.}

Turdus naevius Gmel., Syst. nat., 1788, vol. 1, pt. 2, p. \$17. "in sine Aimericae Natca."

Orpheus meruloides (Swains.). Swainson and Richardson, Fauna Boreali-Amer., 1831, vol. 2, p. 187, pl. 38.

Evergreen forests; nests in trees.

Mass.-Accidental visitor: Ipswich, Dec. 1864.

402. Sialia sialis (Linné) Haldeman.

\section{Bluebird.}

Motacilla sialis Linné, Syrst. nat., ed. 10, 1758, vol. 1, p. 187. "in Bermidis \& Anerica Calidiore."

Sialia wilsonii Swains. Audubon, Birds of Amer., 1St1, vol. 2, p. 171, pl. 134. Eggg, Capen, 1856, pl. 1, figs. 14, 15.

Orehards and open country; nests in hollow limbs or in bird houses.

Ne.-Common migrant and summer resident. (Feb. 28) Mar. 10-Nor. 14. Egrgs, April 21-May 15; June 24-July 6 (2d brood).

N. H.- Common migrant and less common summer resident at lower elevations. Mar. 2-Oct. 25. Eggs, May 1-.June 25. 
PASSERES.

OSCINES.

TURDIDAE.

Tr.-Common migrant and summer resident. (Feb. 23) Mar. 17-Oct. 16 .

MAss.-Common migrant, less common summer resident; near coast, a rare winter resident (Melrose Highlands, Roxbury, Wellesley, Cape Cod). (Feb. 23) Mar. 1-Nor. 20 (Dec. 10, 29); winter. Eggs, April 15-June 5.

R. I.- Common migrant, less common summer resident, and occasional winter resident. Mar. 2-Nor. 27; winter. Eggs, (Mar. 1, Westerly) April 7-20.

Cons.- Common migrant and summer resident; uncommon winter resident. Mar. 5-Nor. 13; winter. Eggs, April 15June 14.

\section{Species Introduced or Erroneously Accredited.}

Colymbus cristatus Linné. Crested grebe.-An Old World species erroneously recorded by early writers.

Aethia cristatella (Pallas). Crested auklet.- Doubtfully recorded from Chatham, Mass., winter of 1884-85.

Cepphus Mandil (Lichtenstein). Mandt's guillemot.-The supposed Maine specimen proves to be $C$.grylle, and the Massachusetts record is too doubtful for acceptance.

Puffinus puffinus (Brïnich). Manx shearwater.

Puffinus "Cinereus" (= kuhl Gould). Mediterranean shearwater.European species listed from New England by several of the older writers but probably in error for $P$. gratis or $P$. borealis.

Thalassidroma pelagica (Linné). Stormy petrel.-Given on authority of Boardman as a rare summer visitor "off the coast of Eastern Maine," but the record is not allowed by Knight.

Pelagodrona marina (Latham). White-faced petrel.-A specimen taken in lat. $40^{\circ} 34^{\prime} 18^{\prime \prime} \mathrm{N}$., long. $66^{\circ} 09^{\prime} \mathrm{W}$., Sept. 2, 1S85, was included in Dr. J. A. Allen's list of Massachusetts birds (1856), but the locality is outsicle the limits covered by the present catalogue.

Phalacrocorax graculus (Limné). Green cormorant.-This European species is accredited to Massachusetts by W. B. O. Pcabody, who doubtless confused it with the American P. auritus. 
Mergellus albeliuds (Linné). Smew.- Accredited by Samucls to Massachusetts, but apparently without good reason

Chenalopex aegreptacus (Linné) Stephens.

\section{Egyptian goose.}

Salvadori, Cat. birds British mus., 1895, vol. 27, p. 169; Gould, Birds of Europc, 1837, vol. 5, pl. [353]. Egg, Scebohm, 1896, p. 36, pl. 12, fig. 1.

Anas aegyptiaca Linné, Syst. nat., ed. 12, 1766, vol. 1, pt. 1, p. 197. "IN AEgY'PTO?"

Rivers and marshes; nests on the ground.

MAss. - The several instances of capture may relate to cither accidental visitors or escapes.

ARdea occidentalis Audubon. Great white heron.- Recorded from Massachusetts in error for Herodias egretta.

Scolopax RUSTicola Linné. European woodcock.- Doubtful reference to this species at Nerport, R. I.; ? Mainc.

Totanus nebularius (Gumnerus). Greenshank.- This Old World specics was accredited to Connecticut by Linsley who states that a specimen was killed at Stratford in fall of $18+2$.

Oreortyx pictus (Douglas) Baird.

\section{Mountain partridge; Plumed partridge.}

Ortyx picta Dougl., Trans. Linn. soc. London, 1829, vol. 16, p. 143. "INTERIOR OF CALIFORNia."

Ortix plumifera Gould. Audubon, Birds of Amer., vol. 5, p. 69, pl. 291. Thickets and open woods; nests on the ground.

Mass - Introduced unsuccessfully about 1890.

Lophortyx CALIFORNicus (Shaw) Bonaparte.

\section{California partridge or quail.}

Tetrao californicus Shaw, Naturalist's miscellany, 1797 (?), vol. 9, pl. 345 and text. "California."

Callipepla californica (Shaw). Gould, Monogr. of Odontophorinae No. Amer., 1850, p. [57], pl. [16]. Egg, Bendire, 1892, vol. 1, pl. 1, fig. 8-10.

Bushy lowlands; nests on the ground.

Mass.- Unsuccessfully introduced in Berkshire Co. about 1890.

Conv.- Introduced: Bridgeport, Dec. 1840.

Lophortyx GaMneli Gambel.

\section{Gambel's partridge.}

Gambel (ex Nuttall), Proc. acad. nat. sci. Phila., 1843, p. 260. "west of California." 
Callipepla gambelii (Nuttall). Cassin and Stephens, Illustr. birds of Calif., Texas, 1853 , no. 2, p. 45 , pl. 9. Egg, Bendire, 1892, vol. 1, pl. 1, fig. 11-14.

Bushy growth; nests on the ground.

MAss. - Unsuccessfully introduced in Berkshire and Bristol Cos. in 1893; Nartha's Vineyard in 1890; Winchendon in 1891.

Tetrao urogallus Linné.

Capercaillie; Muscovian black gamecock; Wood grouse.

Limé, Syst. nat., ed. 10, 1758, vol. 1, p. 159. "IN Europae Frigidae Pinetis paludosis." Ogilvie-Grant, Cat. birds British mus., 1893, rol. 22, p. 62. Dresser, Birds of Europe, 1871-\$1, vol. 5, pl. [489], p. [490]. Egg, Seebohm, 1S96, p. 274, pl. 59, fig. 11.

Mountainous forests; nests on the ground.

Vr.- Introduced about 1897 at Middlebury, Rutland, and elsewhere, unsuccessfully.

Tympanuchus anericanus (Reichenbach) Ridgway.

\section{Prairie hen; Prairie chicken.}

Cupidonia americanus Reich., Syst. avium, 1852, p. xxix. No locality.

Tetrao eupido Limné. Audubon, Birds of Amer., 1S42, vol. 5, p. 93, pl. 296. Egg, Bendire, 1892, vol. 1, pl. 2, fig. 1S-20.

Grassy prairies; nests on the ground.

N. H.- Unsuccessfully introduced at Blue IIt. Park about 1893.

Msss.- Unsuccessfully introduced: Barnstable and Berkshire Cos. in the 'S0's; between Concord and Belmont in 1884 or 1885; Martha's Vineyard, 1902; Naushon Id.; Springfield, 1 S93.

R. I.- Unsuccessfully introduced about 1897.

Pedioecetes phasianellus (Linné) Elliot.

\section{Sharp-tailed grouse.}

Baird, Brewer, and Ridgway, No. Amer. birds, 1574, vol. 3, p. 434, pl. 9, fig. 3 (head).

Tetrao phasianellus Linné, Syst. nat., ed. 10, 1758, vol. 1, p. 160. "CANADA."

Pediaeeactes phasianellus (Limé). Elliot, Monogr. of Tetraoninae, 1865, pl. 15. Egg, Bendire, 1892, vol. 1, pl. 3, fig. 3-5.

Thickets and clearings; nests on the ground.

YT.- Unsuccessfully introduced about 1897.

MAss.- Unsuccessfully introduced near Westport about 1892. 


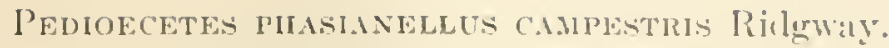

\section{Prairie sharp-tailed grouse.}

Rideway, l'roc. biol. soe. Washington, 18St, rol. 2, p. 93. "Illixols,

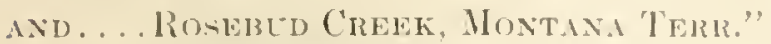

Tetrao phasianallus Limé. Aulubon, Birls of Amer., 15t2, vol. 5, p. 110, pl. 29s Eirg, Bendire, 189?2, vol. 1, pl. 3, figs. 9. 10.

Wooks and thiekets; nests on the ground.

MAss.- Linsuecessfully introdueed at s'pringfiedd, 1s!'?.

COTLRNA CoturNix (Limné) Lichtenstein.

\section{European quail; Migratory quail.}

Ogilvie-Cirant. Cat. birds British mus., 1893, vol. 22, 1). 23.5.

Tetrau coturnix Linné, Syst. nat., ecl. 10, 175s, rol. 1, p. 161. "Evrop', Asis, Arkicı."

C'oturni.r communis Bonnaterre. Dresser, Birls of Europe, 1\$71-\$1, rol. 7 , pl. [476]. Egg, Sechohm, 1s96, p. 277, pl. 59, figs. 1, 3.

Iearlows and fichds; nests on the ground.

Ils.- Introduced unsuceessfully in 1580; 2600 liberated, but failed to establish themselves. Nest at Fahouth, June.

VT.-I"nstrecessully introduced at Rutland, June, 1ST.

MAss-- Insuccessfully introdueed at various plaees (No. Fahnouth, Springfield, Wareham) between 1870 and 1890 .

Coxx.- I'nsuccessully introduced: Lakerille, 1Sis.

Perdix perdix (Limné).

\section{European partridge; Hungarian partridge; Partridge.}

Tetrao perdix Limn", Syst. mat., ed. 10, 1758, rol. 1, p. 166. "Ix EvтroPAE AGIRIS."

Perdir cineren Lathanı. Dresser, Birds of Europe, 1S71-S1, vol. $\boldsymbol{7}, \mathrm{p}$. [131], pls. [474, 470$]$.

Open conntry and thickets; nests on the ground.

Conv- Introdued in 1908 .

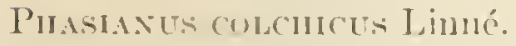

\section{English pheasant; Pheasant.}

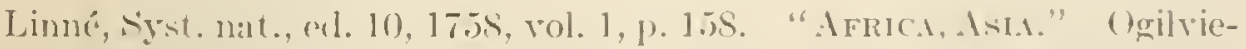
Grant, Cat birds British mus., 1893, vol, 22, p. 321. Dresser, Birds of

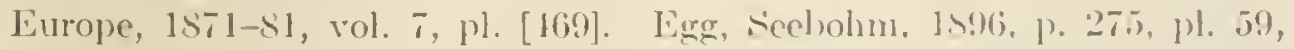
fig. 9. 
Busliy woods; nests on the ground, rarely in trees.

N. H.- Introduced from England at Wolfboro in 1Sth century.

Mass.- Unsuccessfully introduced in Berksh re Co.

Phasianus torquatus Gmelin.

\section{Ring-necked pheasant; Denny pheasant; Ring pheasant.}

Gmel., Syst. nat., 17SS, vol. 1, pt. 2, p. 742. Refers to Latham 'S Synopsis: "China.... about the Casplan Sea,... Great Tartary, and in the south of the Mongolin Desert." Ogilvie-Grant, Cat. birds British mus., 1593, vol. 22, p. 332. Elliot, Monogr. Phasianiclae, 1S72, vol. 2 , pl. 5 .

Thickets; nests on the ground.

VT.-- Introduced successfully at Shelburne.

MASS.- Introduced at Winchester and elsewhere, since 1594, and now well established at many places, mainly in eastern part.

Conv. - Snccessfully introduced (Fisher's Id.).

Columba donestica Gmelin.

\section{Rock dove; Domestic pigeon; Dove; Pigeon.}

Gmel., Srst. nat., 178s, vol. 1, pt. 2, p. 769. No locality.

Columba livia Linné. Dresser, Birds of Europe, 1S71-\$1, vol. 7, p. [11], pl. [457] (upper fig., wild bird). Tegetmeier, Pigeons: their structure, varieties, habits, and management, London, 1S6S, 190 pp., 16 pls. Egg, Seebohm, 1896, p. 158, pl. 47, fig. 9 .

Sea cliffs, nesting in crevices of rocks; now domesticated about buildings.

ME.-Cons.- Introduced under domestication but now common in a feral state in towns and cities.

Buteo borealis harlani (Audubon). Harlan's hawk. Accredited to Massachusetts by Peabody on Nuttall's authority, probably in error.

"Buteo Cooperi" Cassin. Cooper's hen-hawk; California hawk. A record for Cambridge, Mass., is believed to have been based on a very light-colored example of $B$. lincatus.

Surnia ulula (Limné). European hawk owl.- Specimens of this and the American race are said to have been taken at Houlton, Me., in 1S77, but probably all were S. u. caparoch.

Dryobates villosus Leucomelas (Boddaert). Northern hairy woodpecker.- Under this name Chadboume lists the Hairy woodpecker from Williamstown, Mass., probably in error for D. villosus. 
Sphyrapicus varius nuchalis Baird. Red-naped sapsucker.-The supposed New Hampshire and Massachusetts speeinens prove to be S. varius varius.

Agirtria viridssima (Lesson). Linne's hummingbird.-- The authenticity of the supposed Cimbridge specimen is open to doubt.

AlaUda ariensis Linmé.

Skylark; Laverock.

Linné, Syst. nat., ed. 10, 1758, vol. 1, p. 165. "IN Europae Apricis." Ridgway, Bull. 50, U. S. nat. mus., 1907, pt. 4, p. 292; Dresser, Birds of Europe, 1871-81, vol. 4, pl. [231]. Egg, Bendire, 1895, vol. 2, pl. 5, fig. 23.

Open country, fields and meadows; nests on the ground.

Mass.- Unsuccessfully introduced from Europe at Cambriclge, prior to 1880 .

Munia orvzryora (Linné). Java sparrow. - A specimen was taken at Belmont, Mass., Nov. 2, 185t, but this and others are doubtless escaped cage birds.

MUnia atricapilia (Vieillot). Rufous-and black weaver finch. - A specimen of this Indian species, (doubtless an escape), was captured near Brunswick, Maine, in March, 1879 (recorded as Amadina rubronigra).

Sturnelta Neglecta Audubon. Western meadowlark.-Doubtfully recorded from Taunton, Mass.

MEgaquiscalus major (Vieillot). Boat-tailed grackle-Formerly accredited to Maine, Massachusetts, and Connecticut but probably the Bronzed grackle was the species observed.

Serinus butrracea (Limé) Bonaparte.

\section{Yellow finch.}

Fringilla butyracea Linné, Syst. nat., ed. 10, 1758, vol. 1, p. 181. "MADERA."

Serinus flaviventris (Swains.). Sharpe, Cat. birds British Mus., 1S88, vol. 12, p. 353 .

A South Afriean species.

MAss. - Accidental visitor or escape: South Scituate, Feb. 1879, with flock of Redpolls.

Serinus serinus (Linné) Sharpe.

\section{Serin finch.}

Sharpe, Cat. birds British mus., 1888, vol. 12, p. 369. 
Fringilla scrinus Limné, Syst. nat., ed. 12, 1766, rol. 1, pt. 1, p. 320. "IN Europa a t'strali."

Serinus hortulanus Lioch. Dresser, Birds of Europe, 1S71-S1, vol. 3, [pl. 170]. Egg, Seebohm, 1896, p. 240, p]. 56, fig. 16.

Open country, orchards and gardens; nests in trees.

Msass-Probably escaped cagebird: Springfield, Nov. 1866.

Passer domesticus (Limné) Koch.

\section{House sparrow; English sparrow; European house sparrow.}

Ridgway. Birds No. and Niddle Amer., Bul1. 50, U. S. nat. nus., 1901, pt. 1. p. 143. Dresser, Birds of Europe, 1s76, rol. 3, p. [587], pl. [176], fig. 1. Egg, Seebohm, 1s96, p. 23s, pl. 56, fig. 10-12.

Fringilla domestica Linné, Syst. nat., ed. 10, 175\$, rol. 1, p. 1S3. "IN Europa prope rura."

Villages and cities in vieinity of dwellings; nests in trees, vines, or any carity.

ME.-Common resident; introduced at Portland in 1854 and 1858, at Lewiston in 1874, at Bangor about 1875 .

N. H.- Resident, common about large cities, less common in White Mt. valleys.

VT.-Common resident about towns and cities; introduced at St. Johnsbury between 1854 and 1876 .

MAss.-Common resident about towns and eities; introduced at Boston in 18.5 and 1868. Brookline, 1868, Charlestown, 1869, Cottage $\mathrm{C}$ ty about 1S82, Somerville, 1871 .

R. I.-Common resident; introduced at Peacedale in 185s, Providence in 1866 or before, Newport, 1874.

Coxx.-Common resident; introduced at New Haven in 1867, Norwich and Meriden between 1865 and 1870, Portland, 1869.

ACANTHıs bREWStern (Ridgway).

\section{Brewster's linnet.}

Brewster, Mem. Nuttall ornith. club, 1906, no. $t$, pl. 6.

Aegiothus (flarirostris var.) breusterii Ridgw., Amer. nat., 1S72, vol. 6, p. 433. "Wilthan, Massachusetts."

Status uncertain; probably a hybrid between Acanthis linaria and Spinus pinus.

MAss.-Only known specimen taken at Waltham, Nov. 1, 1870.

Cardtelis carduels (Limné) Schäffer.

\section{European goldfinch.}

Ridgray. Bull. 50, U. S. nat mus., 1901, pt. 1, p. 94. Egg, Secbohm, 1896 , p. 241, pl. 56, fig. 17 . 
Fringilla carduelis Linne, syst. nit., ed. 10, 1758, vol. 1, p. 180. "1. EURop.AE JUNiprets."

Curduclis clegans Steplı. Dresser, Birds of Europe, 1571-\$1, vol. 3, pl. [166].

Open country, orchards, and deciduous woods; nests in trees.

MAss. - Introduced in eastern part, but not well established; bred near Worcester, July 11, 1890.

Coxx- Eseape, or straggler from lots introduced in neighboring country: New Haven, May 9, 1892.

Rrachoplanes macownil (Lawrence). McCown's longspur.- Doubtfully recorded for Massachisetts on the basis of a market specinen said to have been taken at Ipswich, Jan. $7,1877$.

Coturniculus Bardi (Audubon). Baird's sparrow.- The first known specimens of Passerculus princeps were recorded under this name.

Peucaed aestivalis Bachumil (Audubou). Bachman's finch.Recorded from Berlin, Mass., in error for Ammortramus henslowii.

PAsserint CIRIs (Limné) Vieillot.

\section{Painted buning; Nonpareil.}

Emberiza ciris Limé, syst. nat., ed. 10, 1758, vol. 1, p. 179. "AnERICA." Spiza ciris (Wils.). Audubon, Birds of Amer., 1841, vol. 3, p. 93, pl. 169. Thickets and hedges; nests in bushes or low trees.

NE.- "Escape," Portland, June 10, 1904.

Mass.- Four instances; believed to be escaped cape birds.

R. I.- Aceidental visitor or escape: Seituate, summer, 1892.

Paroaria céculata (Latham). Crested cardinal. - A South American species, once taken at Newport, R. I., Sept., 1S5.5, but doubtless an escaped cagebird.

Gurernatrix cristata (Vieillot). - A South Ameriean finch, of which a specimen supposed to be an eseape, was taken at Providence. R. I., July $7,18 s$.

Vireo atricipillus Woodhouse. Black-capped Vireo.- Very doubtfully recorded from Laneshoro, Mass.

Helinala sWansonil (Audubon). Swainson's warbler.- Erroneously aceredited to Massachusetts by Audubon.

Helintiophila LAWRexcei (Herrick) Riclgway.

Lawrence's warbler.

Ridgway, Bull. 50, U. S. nat. mus., 1902, pt. 2, p. 452. 
Hclminthophaga lawrencii Herrick, Proc. acad. nat. sci. Phila., 187t, p. 220, pl. 15. "Near Cinathair, New Jersey."

Probably a hybrid form between $H$. pinus and $H$. chrysoptera, which it resembles in habits.

MAss.- One recorded as seen at Dedham, May 27, 1908.

Cosx.-Summer resident: Bridgeport, New Haren, Portland, Stamford. May 12-July. Egrgs, June 14.

Helimthophila leucobroxchialis (Brewster) Ridgway.

\section{Brewster's warbler; White-throated warbler.}

Helminthophaga Teucobronchialis Brewst., Bull. Nuttall ornith. club, 1S76, rol. 1, p. 1, pl. 1. "Newtonville, Mass."

Probably a hybrid form between $H$. pinus and $H$. chrysoptcra, which it resembles in habits.

Mass.- Rare summer resident in eastern part (Hudson, Lexington, Nertonville, Jamaica Plain). May 14-summer. Eggs, June 8-18.

Conx.-- Rare summer resident. May 6-Aug. S. Eggs, June 24.

"Muscicapa Minuta" Wilson = Wilsonia microcephala (Rirlgway). Small-headed flycatcher. An hypothetical species, described by Wilson and accredited to Massachusetts by Nuttall, Brewer, and Peabody.

Penthestes atricapillus septentrionalis (Harris). Long-tailed chickadee. Erroneously recorded from Rhode Island; the specimen proves to be $P$. a. atricapillus.

Srluia atricapilla (Linné) Scopoli.

\section{European blackcap warbler; Blackcap.}

Dresser, Birds of Europe, 1871-S1, vol 2, p. [421], pl. [66]. Egg, Seebohm, 1896, p. 201, pl. 52, fig. 17-19.

Motacilla atricapilla Linné, Syst. nat., ed. 10, 1758, vol. 1, p. 1S7. "IN Europa."

Grores and orchards; nests in bushes.

Mass.- Unsucessfully introduced at Cambridge prior to 1877.

Turdus pilaris Linné. Fieldfare.-A European species, once taken near Stamford, Conn., Mar. 1S\$S, but this probably an escaped cagebird.

Planesticus merula (Linné). Blackbird.-A male, probably an escape, spent the writer of 1908-09 near the Boston public garden.

Saxicola ofinthe leucorhoa (Gmelin). Greenland wheatear.The supposed Maine records in reality refer to birds taken across the line in New Brunswick. 






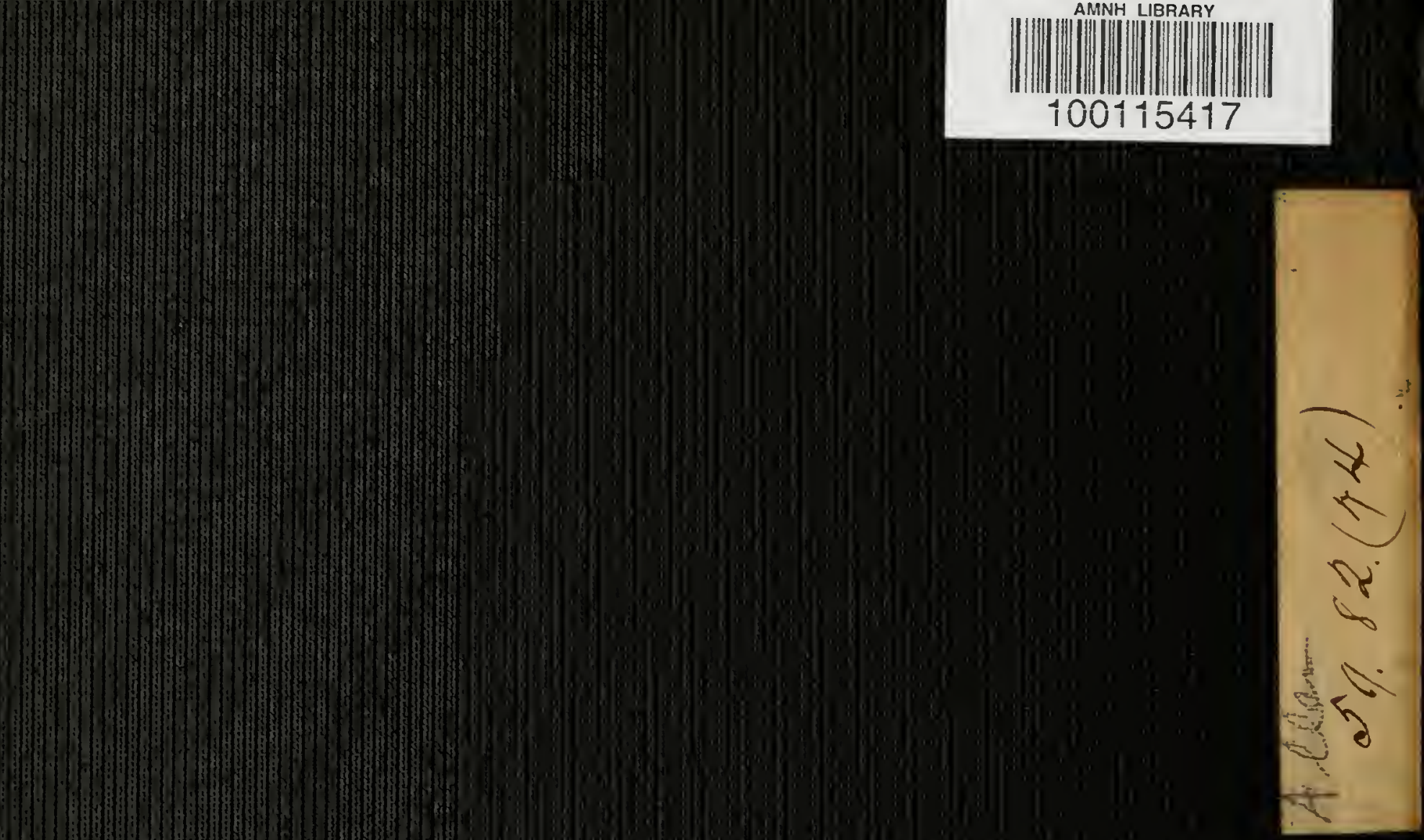